\title{
Xpert MTB/RIF and Xpert MTB/RIF Ultra for pulmonary tuberculosis and rifampicin resistance in adults (Review)
}

Horne DJ, Kohli M, Zifodya JS, Schiller I, Dendukuri N, Tollefson D, Schumacher SG, Ochodo EA, Pai M, Steingart KR

Horne DJ, Kohli M, Zifodya JS, Schiller I, Dendukuri N, Tollefson D, Schumacher SG, Ochodo EA, Pai M, Steingart KR. Xpert MTB/RIF and Xpert MTB/RIF Ultra for pulmonary tuberculosis and rifampicin resistance in adults.

Cochrane Database of Systematic Reviews 2019, Issue 6. Art. No.: CD009593.

DOI: 10.1002/14651858.CD009593.pub4.

www.cochranelibrary.com 
TABLE OF CONTENTS

HEADER

ABSTRACT

PLAIN LANGUAGE SUMMARY .

SUMMARY OF FINDINGS

BACKGROUND

Figure 1.

Figure 2.

OBJECTIVES

METHODS

RESULTS

Figure 3.

Figure 4.

Figure 5.

Figure 6.

Figure 7.

Figure 8.

Figure 9.

Figure 10.

Figure 11.

Figure 12.

Figure 13.

Figure 14.

Figure 15.

Figure 16.

Figure 17.

Figure 18.

Figure 19.

Figure 20.

Figure 21.

DISCUSSION

AUTHORS' CONCLUSIONS

ACKNOWLEDGEMENTS

REFERENCES

CHARACTERISTICS OF STUDIES

DATA

Test 1. Xpert MTB/RIF for detection of pulmonary tuberculosis (PTB).

Test 2. Xpert Ultra for detection of PTB.

Test 3. Smear-positive, Xpert MTB/RIF.

Test 4. Smear-positive, Xpert MTB/RIF, direct comparison Xpert MTB/RIF vs Xpert Ultra.

Test 5. Smear-positive, Xpert Ultra, direct comparison Xpert MTB/RIF vs Xpert Ultra.

Test 6. Smear-negative, Xpert MTB/RIF.

Test 7. Smear-negative, Xpert MTB/RIF, direct comparison Xpert MTB/RIF vs Xpert Ultra.

Test 8. Smear-negative, Xpert Ultra, direct comparison Xpert MTB/RIF vs Xpert Ultra.

Test 9. HIV-negative, Xpert MTB/RIF.

Test 10. HIV-positive, Xpert MTB/RIF.

Test 11. HIV-negative, within study comparisons.

Test 12. HIV-positive, within study comparisons.

Test 13. HIV-negative, Xpert MTB/RIF, direct comparison Xpert MTB/RIF vs Xpert Ultra.

Test 14. HIV-negative, Xpert Ultra, direct comparison Xpert MTB/RIF vs Xpert Ultra.

Test 15. HIV-positive, Xpert MTB/RIF, direct comparison Xpert MTB/RIF vs Xpert Ultra.

Test 16. HIV-positive, Xpert Ultra, direct comparison Xpert MTB/RIF vs Xpert Ultra. 


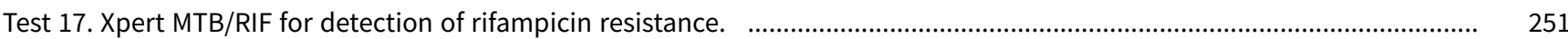

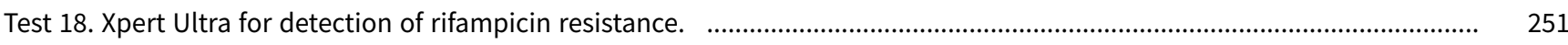

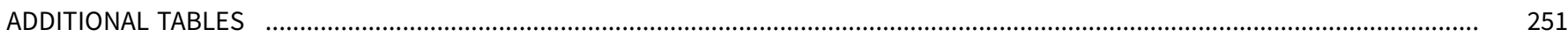

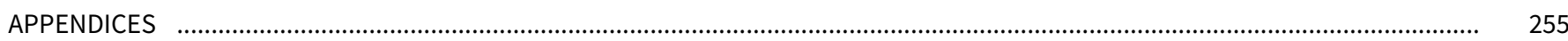

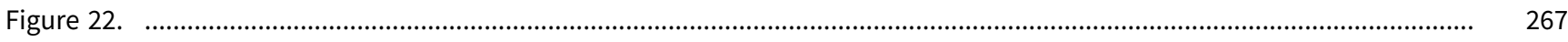

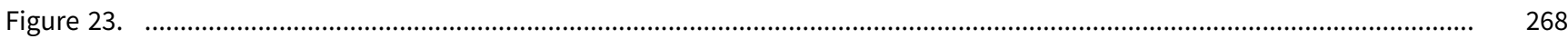

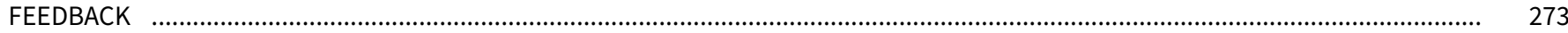

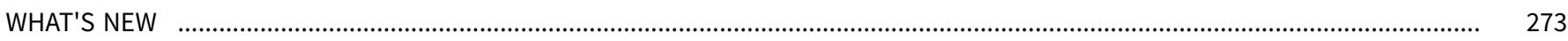

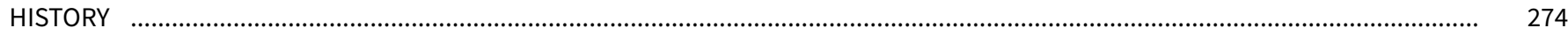

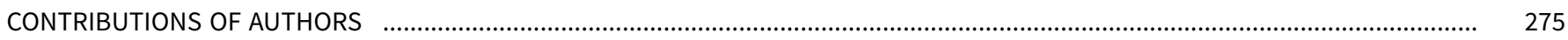

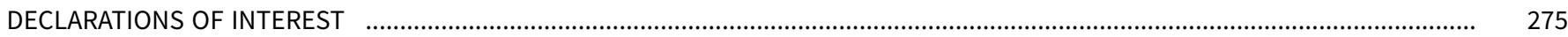

SOURCES OF SUPPORT

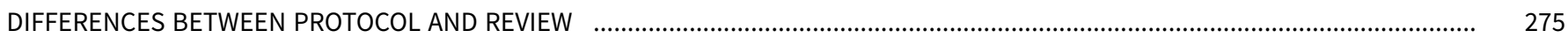

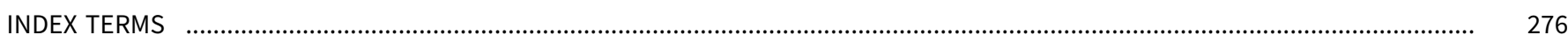




\section{Xpert MTB/RIF and Xpert MTB/RIF Ultra for pulmonary tuberculosis and rifampicin resistance in adults}

David J Horne ${ }^{1 a}$, Mikashmi Kohli2b, Jerry S Zifodya ${ }^{3}$, Ian Schiller ${ }^{4}$, Nandini Dendukuri4 ${ }^{4}$, Deanna Tollefson 5 , Samuel G Schumacher 6 , Eleanor A Ochodo 7 , Madhukar Pai ${ }^{2}$, Karen R Steingart 8

1Department of Medicine, Division of Pulmonary and Critical Care Medicine, and Firland Northwest TB Center, University of Washington, Seattle, WA, USA. ${ }^{2}$ Department of Epidemiology, Biostatistics and Occupational Health, McGill University, Montreal, Canada. ${ }^{3}$ Pulmonary and Critical Care Medicine, University of Washington, Seattle, WA, USA. ${ }^{4}$ Division of Clinical Epidemiology, McGill University Health Centre - Research Institute, Montreal, Canada. ${ }^{5}$ Department of Global Health, University of Washington, Seattle, WA, USA. 6 FIND, Geneva, Switzerland. ${ }^{7}$ Centre for Evidence-based Health Care, Faculty of Medicine and Health Sciences, Stellenbosch University, Cape Town, South Africa. ${ }^{8}$ Honorary Research Fellow, Department of Clinical Sciences, Liverpool School of Tropical Medicine, Liverpool, UK

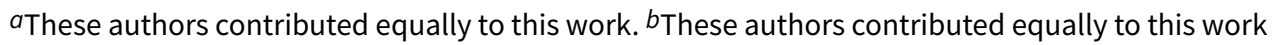

Contact address: Karen R Steingart, Honorary Research Fellow, Department of Clinical Sciences, Liverpool School of Tropical Medicine, Pembroke Place, Liverpool, UK. karen.steingart@gmail.com.

Editorial group: Cochrane Infectious Diseases Group

Publication status and date: Unchanged, published in Issue 6, 2019.

Citation: Horne DJ, Kohli M, Zifodya JS, Schiller I, Dendukuri N, Tollefson D, Schumacher SG, Ochodo EA, Pai M, Steingart KR. Xpert MTB/ RIF and Xpert MTB/RIF Ultra for pulmonary tuberculosis and rifampicin resistance in adults. Cochrane Database of Systematic Reviews 2019, Issue 6. Art. No.: CD009593. DOI: 10.1002/14651858.CD009593.pub4.

Copyright (C) 2019 The Authors. Cochrane Database of Systematic Reviews published by John Wiley \& Sons, Ltd. on behalf of The Cochrane Collaboration. This is an open access article under the terms of the Creative Commons Attribution-Non-Commercial Licence, which permits use, distribution and reproduction in any medium, provided the original work is properly cited and is not used for commercial purposes.

\section{A B S T R A C T}

\section{Background}

Xpert MTB/RIF (Xpert MTB/RIF) and Xpert MTB/RIF Ultra (Xpert Ultra), the newest version, are the only World Health Organization (WHO)recommended rapid tests that simultaneously detect tuberculosis and rifampicin resistance in persons with signs and symptoms of tuberculosis, at lower health system levels. A previous Cochrane Review found Xpert MTB/RIF sensitive and specific for tuberculosis (Steingart 2014). Since the previous review, new studies have been published. We performed a review update for an upcoming WHO policy review.

\section{Objectives}

To determine diagnostic accuracy of Xpert MTB/RIF and Xpert Ultra for tuberculosis in adults with presumptive pulmonary tuberculosis (PTB) and for rifampicin resistance in adults with presumptive rifampicin-resistant tuberculosis.

\section{Search methods}

We searched the Cochrane Infectious Diseases Group Specialized Register, MEDLINE, Embase, Science Citation Index, Web of Science, Latin American Caribbean Health Sciences Literature, Scopus, the WHO International Clinical Trials Registry Platform, the International Standard Randomized Controlled Trial Number Registry, and ProQuest, to 11 October 2018, without language restriction.

\section{Selection criteria}

Randomized trials, cross-sectional, and cohort studies using respiratory specimens that evaluated Xpert MTB/RIF, Xpert Ultra, or both against the reference standard, culture for tuberculosis and culture-based drug susceptibility testing or MTBDRplus for rifampicin resistance. 


\section{Data collection and analysis}

Four review authors independently extracted data using a standardized form. When possible, we also extracted data by smear and HIV status. We assessed study quality using QUADAS-2 and performed meta-analyses to estimate pooled sensitivity and specificity separately for tuberculosis and rifampicin resistance. We investigated potential sources of heterogeneity. Most analyses used a bivariate random-effects model. For tuberculosis detection, we first estimated accuracy using all included studies and then only the subset of studies where participants were unselected, i.e. not selected based on prior microscopy testing.

\section{Main results}

We identified in total 95 studies (77 new studies since the previous review): 86 studies (42,091 participants) evaluated Xpert MTB/RIF for tuberculosis and 57 studies (8287 participants) for rifampicin resistance. One study compared Xpert MTB/RIF and Xpert Ultra on the same participant specimen.

\section{Tuberculosis detection}

Of the total 86 studies, 45 took place in high tuberculosis burden and 50 in high TB/HIV burden countries. Most studies had low risk of bias.

Xpert MTB/RIF pooled sensitivity and specificity (95\% credible Interval (Crl)) were $85 \%$ (82\% to $88 \%$ ) and $98 \%$ (97\% to $98 \%$ ), (70 studies, 37,237 unselected participants; high-certainty evidence). We found similar accuracy when we included all studies.

For a population of 1000 people where 100 have tuberculosis on culture, 103 would be Xpert MTB/RIF-positive and 18 (17\%) would not have tuberculosis (false-positives); 897 would be Xpert MTB/RIF-negative and 15 (2\%) would have tuberculosis (false-negatives).

Xpert Ultra sensitivity (95\% confidence interval (CI)) was 88\% (85\% to 91\%) versus Xpert MTB/RIF 83\% (79\% to 86\%); Xpert Ultra specificity was $96 \%$ (94\% to $97 \%$ ) versus Xpert MTB/RIF 98\% (97\% to 99\%), (1 study, 1439 participants; moderate-certainty evidence).

Xpert MTB/RIF pooled sensitivity was $98 \%$ (97\% to $98 \%$ ) in smear-positive and $67 \%$ (62\% to $72 \%$ ) in smear-negative, culture-positive participants, (45 studies). Xpert MTB/RIF pooled sensitivity was $88 \%$ (83\% to $92 \%$ ) in HIV-negative and $81 \%(75 \%$ to $86 \%)$ in HIV-positive participants; specificities were similar $98 \%$ (97\% to $99 \%)$, (14 studies).

\section{Rifampicin resistance detection}

Xpert MTB/RIF pooled sensitivity and specificity (95\% Crl) were $96 \%$ (94\% to 97\%) and 98\% (98\% to 99\%), (48 studies, 8020 participants; high-certainty evidence).

For a population of 1000 people where 100 have rifampicin-resistant tuberculosis, 114 would be positive for rifampicin-resistant tuberculosis and 18 (16\%) would not have rifampicin resistance (false-positives); 886 would be would be negative for rifampicin-resistant tuberculosis and four (0.4\%) would have rifampicin resistance (false-negatives).

Xpert Ultra sensitivity $(95 \% \mathrm{CI})$ was $95 \%(90 \%$ to $98 \%$ ) versus Xpert MTB/RIF $95 \%$ ( $91 \%$ to $98 \%)$; Xpert Ultra specificity was $98 \%$ ( $97 \%$ to 99\%) versus Xpert MTB/RIF 98\% (96\% to 99\%), (1 study, 551 participants; moderate-certainty evidence).

\section{Authors' conclusions}

We found Xpert MTB/RIF to be sensitive and specific for diagnosing PTB and rifampicin resistance, consistent with findings reported previously. Xpert MTB/RIF was more sensitive for tuberculosis in smear-positive than smear-negative participants and HIV-negative than HIVpositive participants. Compared with Xpert MTB/RIF, Xpert Ultra had higher sensitivity and lower specificity for tuberculosis and similar sensitivity and specificity for rifampicin resistance (1 study). Xpert MTB/RIF and Xpert Ultra provide accurate results and can allow rapid initiation of treatment for multidrug-resistant tuberculosis.

29 October 2019

Update pending

Authors currently updating

The update is due to be published in 2020.

\section{PLAIN LANGUAGE SUMMARY}

Xpert MTB/RIF and Xpert Ultra for diagnosing pulmonary tuberculosis and rifampicin resistance in adults

\section{Why is improving the diagnosis of pulmonary tuberculosis important?}

Tuberculosis causes more deaths globally than any other infectious disease. When detected early and effectively treated, tuberculosis is largely curable, but in 2017, around 1.6 million people died of tuberculosis. Xpert MTB/RIF and Xpert Ultra, the newest version, are World 
Health Organization-recommended tests that simultaneously detect tuberculosis and rifampicin resistance in persons with tuberculosis symptoms. Rifampicin is an important anti-tuberculosis drug. Not recognizing tuberculosis early may result in delayed diagnosis and treatment, severe illness, and death. An incorrect tuberculosis diagnosis may result in anxiety and unnecessary treatment.

\section{What is the aim of this review?}

To determine how accurate Xpert MTB/RIF and Xpert Ultra are for diagnosing pulmonary tuberculosis (PTB) and rifampicin resistance in adults. This is an update of the 2014 Cochrane Review.

\section{What was studied in this review?}

Xpert MTB/RIF and Xpert Ultra, with results measured against culture (benchmark).

\section{What are the main results in this review?}

95 studies: 86 studies (42,091 participants) evaluated Xpert MTB/RIF for tuberculosis; 57 studies (8287 participants) for rifampicin resistance. One study compared Xpert Ultra and Xpert MTB/RIF.

For PTB, Xpert MTB/RIF was sensitive (85\%), registering positive in people who actually had tuberculosis, and specific (98\%), i.e. it did not register positive in people who were actually negative. Xpert Ultra had higher sensitivity than Xpert MTB/RIF ( $88 \%$ versus $83 \%)$ in one study.

For rifampicin resistance, Xpert MTB/RIF was highly sensitive (96\%) and specific (98\%). Xpert Ultra gave similar results.

Xpert MTB/RIF was better for diagnosing tuberculosis in HIV-negative than in HIV-positive people.

\section{How confident are we in the results of this review?}

Confident. We included many studies and used the best reference standards.

\section{Who do the results of this review apply to?}

People with presumed PTB or rifampicin resistance.

\section{What are the implications of this review?}

In theory, among 1000 people where 100 have tuberculosis on culture, 103 would be Xpert MTB/RIF-positive and 18 (17\%) would not have tuberculosis (false-positives); 897 would be Xpert MTB/RIF-negative and 15 (2\%) would have tuberculosis (false-negatives).

Among 1000 people where 100 have rifampicin resistance, 114 would be positive for rifampicin resistance and 18 (16\%) would not have rifampicin resistance (false-positives); 886 would be negative for rifampicin resistance and four (0.4\%) would have rifampicin resistance (false-negatives).

\section{How up-to-date is this review?}

To 11 October 2018. 


\section{SUMMARY OF FINDINGS}

\section{Summary of findings 1. Xpert MTB/RIF and Xpert Ultra for detection of pulmonary tuberculosis}

Review question: What is the diagnostic accuracy of Xpert MTB/RIF and Xpert Ultra for detection of pulmonary tuberculosis (PTB)?

Patients/population: Adults with presumptive PTB. Participants were 'unselected', meaning they were not enrolled in a study based on microscopy smear results or past history of tuberculosis

Role: An initial test

Index tests: Xpert MTB/RIF and Xpert Ultra

Threshold for index tests: An automated result is provided

Reference standards: Solid or liquid culture

Studies: Cross-sectional and cohort studies

Setting: Primary care facilities and local hospitals

\begin{tabular}{|c|c|c|c|c|c|c|c|}
\hline \multirow[t]{2}{*}{ Index test } & \multirow[t]{2}{*}{ Effect $(95 \% \mathrm{Crl})$} & \multirow{2}{*}{$\begin{array}{l}\text { Number of } \\
\text { participants } \\
\text { (studies) }\end{array}$} & \multirow[t]{2}{*}{ Test result } & \multicolumn{3}{|c|}{ Number of results per 1000 patients tested $(95 \% \mathrm{CrI})^{1}$} & \multirow{2}{*}{$\begin{array}{l}\text { Certain- } \\
\text { ty of the } \\
\text { evidence } \\
\text { (GRADE) }\end{array}$} \\
\hline & & & & Prevalence $1 \%$ & Prevalence $10 \%$ & Prevalence $30 \%$ & \\
\hline \multirow{3}{*}{$\begin{array}{l}\text { Xpert MTB/ } \\
\text { RIF in unse- } \\
\text { lected par- } \\
\text { ticipants }\end{array}$} & \multirow{2}{*}{$\begin{array}{l}\text { Pooled sensitivity } 85 \% \\
\text { ( } 82 \text { to } 88 \text { ) }\end{array}$} & \multirow{2}{*}{$\begin{array}{l}10,409(70 \\
\text { studies })\end{array}$} & True positives & 9 (8 to 9$)$ & 85 (82 to 88$)$ & 255 (246 to 264$)$ & \multirow{2}{*}{$\begin{array}{l}\oplus \oplus \oplus \oplus \\
\text { Higha,b,c }\end{array}$} \\
\hline & & & False negatives & 1 (1 to 2$)$ & 15 (12 to 18$)$ & 45 (36 to 54$)$ & \\
\hline & $\begin{array}{l}\text { Pooled specificity } 98 \% \\
\text { (97 to } 98 \text { ) }\end{array}$ & $\begin{array}{l}26,828(70 \\
\text { studies) }\end{array}$ & False positives & 20 (20 to 30$)$ & 18 (18 to 27$)$ & 14 (14 to 21$)$ & $\begin{array}{l}\oplus \oplus \oplus \oplus \\
\text { High } a\end{array}$ \\
\hline \multirow[t]{3}{*}{ Xpert Ultra } & \multirow{2}{*}{$\begin{array}{l}\text { Sensitivity } 88 \% \text { ( } 85 \text { to } \\
91 \text { ) }\end{array}$} & \multirow[t]{2}{*}{462 (1 study) } & True positives & 9 (9 to 9$)$ & 88 (85 to 91$)$ & 264 (255 to 273 ) & \multirow{2}{*}{$\begin{array}{l}\oplus \oplus \oplus \ominus \\
\text { Moderated,e }\end{array}$} \\
\hline & & & False negatives & $1(1$ to 1$)$ & 12 (9 to 15$)$ & $36(27$ to 45$)$ & \\
\hline & $\begin{array}{l}\text { Specificity } 96 \% \text { (94 to } \\
\text { 97) }\end{array}$ & 977 (1 study) & True negatives & 950 (931 to 960$)$ & 864 (846 to 873$)$ & 672 (658 to 679$)$ & $\begin{array}{l}\oplus \oplus \oplus \ominus \\
\text { Moderated,e }\end{array}$ \\
\hline
\end{tabular}

Abbreviations: Crl: credible interval; PTB: pulmonary tuberculosis.

Prevalence estimates were suggested by the WHO Global TB Programme. For Xpert MTB/RIF, the median tuberculosis prevalence in the included studies was $26 \%$. For Xpert Ultra, the tuberculosis prevalence in the study was $32 \%$. 
Credible limits were estimated based on those around the point estimates for pooled sensitivity and specificity; $95 \%$ confidence intervals were estimated for the single study that evaluated Ultra.

aThe median tuberculosis prevalence in the studies was $26 \%$ and thus the results tend to be more applicable to settings with a higher tuberculosis prevalence. For tuberculosis prevalence of $1 \%$ and $10 \%$, whether or not to downgrade is unclear. It is possible the test will perform differently at lower tuberculosis prevalences. We did not downgrade for indirectness.

bFor individual studies, sensitivity estimates ranged from $43 \%$ to $100 \%$. We thought that differences in enrolment criteria (different populations targeted), disease severity, and setting could in part explain heterogeneity. We did not downgrade for inconsistency.

cThere were a large number of studies and participants in this analysis. The $95 \% \mathrm{Crl}$ around true positives and false negatives would probably not lead to different decisions depending on which credible limits are assumed. We did not downgrade for imprecision.

dThe tuberculosis prevalence in the study was $32 \%$ and thus the results tend to be more applicable to settings with a higher tuberculosis prevalence. For tuberculosis prevalences of $1 \%$ and $10 \%$, whether or not to downgrade is unclear. It is possible the test will perform differently at lower prevalences. We did not downgrade for indirectness.

eAlthough there was only one study on the accuracy of Xpert Ultra for PTB, this was a multicentre study conducted in eight countries (South Africa, Uganda, Kenya, India, China, Georgia, Belarus, and Brazil). We downgraded by one level for imprecision.

\section{GRADE certainty of the evidence}

High: we are very confident that the true effect lies close to that of the estimate of the effect.

Moderate: we are moderately confident in the effect estimate: the true effect is likely to be close to the estimate of the effect, but there is a possibility that it is substantially different.

Low: our confidence in the effect estimate is limited: the true effect may be substantially different from the estimate of the effect.

Very low: we have very little confidence in the effect estimate: the true effect is likely to be substantially different from the estimate of effect.

The results presented in this table should not be interpreted in isolation from results of the individual included studies contributing to each summary test accuracy measure.

\section{Summary of findings 2. Xpert MTB/RIF and Xpert Ultra for detection of rifampicin resistance}

Review question: What is the diagnostic accuracy of Xpert MTB/RIF and Xpert Ultra for detection of rifampicin resistance?

Patients/population: Adults with confirmed PTB

Role: An initial test

Index tests: Xpert MTB/RIF and Xpert Ultra

Threshold for index tests: An automated result is provided

Reference standards: Phenotypic culture-based DST and MTBDRplus

Studies: Cross-sectional and cohort studies

Setting: Primary care facilities and local hospitals

\begin{tabular}{|c|c|c|c|c|c|c|c|}
\hline \multirow[t]{2}{*}{ Index test } & \multirow[t]{2}{*}{ Effect $(95 \% \mathrm{Crl})$} & \multirow{2}{*}{$\begin{array}{l}\text { Number of par- } \\
\text { ticipants (stud- } \\
\text { ies) }\end{array}$} & \multirow[t]{2}{*}{ Test result } & \multicolumn{3}{|c|}{ Number of results per 1000 patients tested $(95 \% \mathrm{Crl})$} & \multirow{2}{*}{$\begin{array}{l}\text { Certain- } \\
\text { ty of the } \\
\text { evidence } \\
\text { (GRADE) }\end{array}$} \\
\hline & & & & Prevalence $5 \%$ & Prevalence $10 \%$ & $\begin{array}{l}\text { Prevalence } \\
15 \%\end{array}$ & \\
\hline $\begin{array}{l}\text { Xpert MTB/ } \\
\text { RIF }\end{array}$ & $\begin{array}{l}\text { Pooled sensitivity } 96 \% \\
\text { (94 to } 97 \text { ) }\end{array}$ & $\begin{array}{l}1775 \text { (48 stud- } \\
\text { ies) }\end{array}$ & True positives & 48 (47 to 49 ) & 96 (94 to 97$)$ & 144 (141 to 146$)$ & $\oplus \oplus \oplus \oplus$ \\
\hline
\end{tabular}




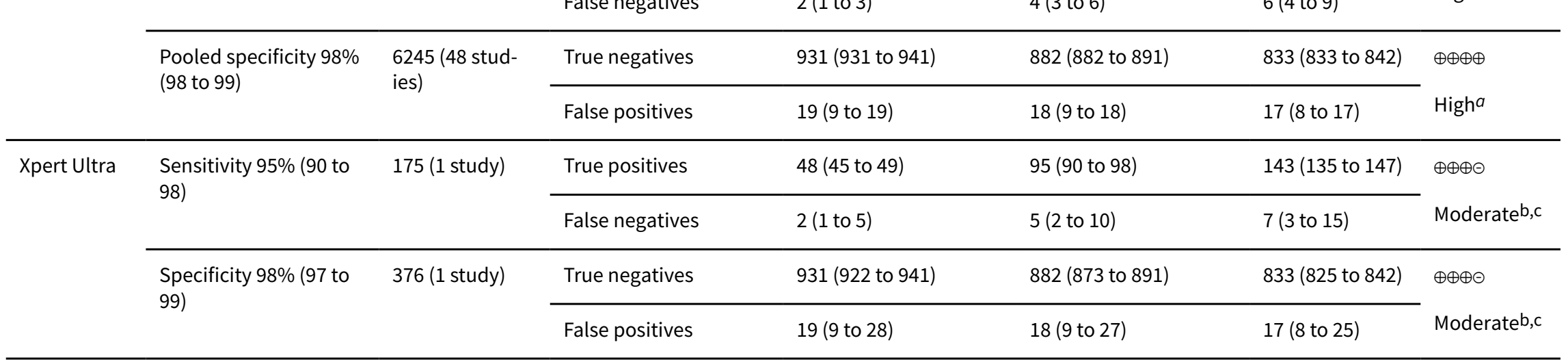

Abbreviations: Crl: credible interval; DST: drug susceptibility testing; PTB: pulmonary tuberculosis.

Prevalence estimates were suggested by the WHO Global TB Programme. The upper limit for the prevalence of rifampicin resistance in new cases was estimated to be $5 \%$ ( $50 / 1000$ cases); the lower limit for the prevalence of rifampicin resistance in previously-treated cases was estimated to be $15 \%$ (150/1000 cases). For Xpert MTB/RIF, the median prevalence of rifampicin resistance in the included studies was $11 \%$. For Xpert Ultra, the prevalence of rifampicin resistance in the study was $32 \%$.

Credible limits were estimated based on those around the point estimates for pooled sensitivity and specificity; $95 \%$ confidence intervals were estimated for the single study that evaluated Xpert Ultra.

aln the Patient Selection domain, with respect to applicability, we had low concern in $46 \%$ of studies and high concern in only $7 \%$ of studies. In nearly half of the studies (47\%) the clinical setting was not reported or there was insufficient information to make a decision. We did not downgrade for indirectness.

bThe prevalence of rifampicin resistance in the study was $32 \%$ (higher than the three prevalence levels considered in the table). Although it is possible that the test will perform differently at lower prevalences, we think that this is unlikely. The magnitude of any effect (either direction) is probably small, given that in this study both Xpert MTB/RIF and Xpert Ultra sensitivity and specificity for rifampicin resistance were nearly identical to the pooled sensitivity and specificity in the review. We did not downgrade for indirectness. cAlthough there was only one study on the accuracy of Xpert Ultra for rifampicin resistance, this was a multicentre study conducted in eight countries (South Africa, Uganda, Kenya, India, China, Georgia, Belarus, and Brazil). We downgraded by one level for imprecision.

\section{GRADE certainty of the evidence}

High: we are very confident that the true effect lies close to that of the estimate of the effect.

Moderate: we are moderately confident in the effect estimate: the true effect is likely to be close to the estimate of the effect, but there is a possibility that it is substantially different.

Low: our confidence in the effect estimate is limited: the true effect may be substantially different from the estimate of the effect.

Very low: we have very little confidence in the effect estimate: the true effect is likely to be substantially different from the estimate of effect.

The results presented in this table should not be interpreted in isolation from results of the individual included studies contributing to each summary test accuracy measure. 


\section{B A C K G R O U N D}

Tuberculosis is the world's leading cause of infectious disease-related death and is one of the top 10 causes of death worldwide (WHO Global TB Report 2018). In 2017, 10 million people developed tuberculosis disease, equivalent to 133 cases per 100,000 population (WHO Global TB Report 2018). Of the 10 million tuberculosis cases, approximately $9 \%$ occurred among people living with HIV. Worldwide, for all forms of tuberculosis, a substantial percentage ( 36\%) of patients were not reported to national treatment programmes (WHO Global TB Report 2018). When tuberculosis is detected early and is effectively treated, the disease is largely curable. However, in 2017, 1.6 million people died of tuberculosis, including 300,000 deaths among people living with HIV (WHO Global TB Report 2018). Ending the tuberculosis epidemic by 2030 is among the health targets of the Sustainable Development Goals.

Drug-resistant tuberculosis is a serious threat to global health (Zumla 2012). Three groupings for tuberculosis drug resistance are used for the purpose of surveillance and treatment: rifampicin-resistant tuberculosis, multidrug-resistant tuberculosis (MDR-TB), and extensively drug-resistant tuberculosis (XDR-TB). MDR-TB is defined as resistance to at least isoniazid and rifampicin, the two most important first-line anti-tuberculosis drugs. XDR-TB is defined as MDR-TB plus resistance to at least one drug in the following two classes of medicines used in treatment of MDR-TB: fluoroquinolones and second-line injectable agents (WHO Global TB Report 2018). In 2017, approximately 558,000 people developed MDR-TB/rifampicin-resistant tuberculosis. Regarding XDRTB, 10,800 cases were reported by 77 countries (WHO Global TB Report 2018). In 2017, 30\% of new and previously-treated people with tuberculosis were tested for rifampicin resistance; while this is a significant improvement over recent rates, considerable gaps remain.

Accurate and rapid detection of tuberculosis, including smear-negative tuberculosis and drug resistant-tuberculosis, is critical for improving patient outcomes (increased cure and decreased mortality, and prevention of additional drug resistance, treatment failure, and relapse), and decreasing tuberculosis transmission. Mycobacterial culture is generally considered the best available reference standard for tuberculosis diagnosis and is a key step in detecting drug resistance. However, culture is a relatively complex and slow procedure. Solid culture typically takes between four to eight weeks for results and liquid culture, although more sensitive and rapid than solid culture, requires weeks and is more prone to contamination (WHO Policy Framework 2015). In addition, culture requires specialized laboratories and highly skilled staff. In 2010, the World Health Organization (WHO) recommended the use of a novel, rapid, automated, cartridge-based, nucleic acid amplification (NAA) test, Xpert MTB/RIF (Cepheid, Sunnyvale, USA) (hereafter referred to as Xpert MTB/RIF), that can simultaneously detect tuberculosis and rifampicin resistance (WHO Policy Xpert MTB/RIF 2011).

\section{Target condition being diagnosed}

\section{Tuberculosis}

Tuberculosis is caused by the bacterium Mycobacterium tuberculosis (M tuberculosis) and is spread from person to person through the air. Tuberculosis most commonly affects the lungs (pulmonary tuberculosis (PTB)), but may affect any organ or tissue outside of the lungs (extrapulmonary tuberculosis). Signs and symptoms of PTB include cough, fever, chills, night sweats, weight loss, haemoptysis (coughing up blood), and fatigue. Signs and symptoms of extrapulmonary tuberculosis depend on the site of disease. Tuberculosis treatment regimens must contain multiple drugs to which the organisms are sensitive to cure tuberculosis and avoid selection for drug resistance. The treatment of MDR-TB is complex, historically requiring two years or more of therapy, although the WHO conditionally recommended a nine- to 12-month regimen in 2016 (WHO 2016b). The drugs used to treat MDR-TB are less potent and more toxic than the drugs used to treat drug-susceptible tuberculosis.

\section{Rifampicin resistance}

Rifampicin inhibits bacterial DNA-dependent RNA polymerase, encoded by the RNA polymerase gene (rpoB) (Hartmann 1967). Resistance to this drug has mainly been associated with mutations in a limited region of the $r p o B$ gene (Telenti 1993). Rifampicin resistance may occur alone or in association with resistance to isoniazid and other drugs. In high MDR-TB settings, the presence of rifampicin resistance alone may serve as a proxy for MDR-TB (WHO Rapid Implementation 2011). People with drug-resistant tuberculosis can transmit the infection to others.

\section{Index test(s)}

Xpert MTB/RIF is an automated polymerase chain reaction (PCR) test (molecular test) using the GeneXpert platform (Blakemore 2010; Cepheid 2009; Helb 2010). Xpert MTB/RIF is a single test that can detect both $M$ tuberculosis complex and rifampicin resistance within two hours after starting the test, with minimal hands-on technical time. Unlike conventional NAA tests, Xpert MTB/RIF is unique because sample processing and PCR amplification and detection are integrated into a single self-enclosed test unit, the Genexpert cartridge. Following sample loading, all steps in the assay are completely automated and self-contained. In addition, the assay's sample reagent, used to liquefy sputum, has potent tuberculocidal (the ability to kill tuberculosis bacteria) properties and so largely eliminates biosafety concerns during the test procedure (Banada 2010). These features allow the technology to be taken out of a reference laboratory and used nearer to the patient (Small 2011). Xpert MTB/RIF requires an uninterrupted and stable electrical power supply, temperature control, and yearly calibration of the cartridge modules (WHO Rapid Implementation 2011).

The test procedure may be used directly on clinical specimens, either raw sputum specimens or sputum pellets created after decontaminating and concentrating the sputum (Blakemore 2010). In both cases, the test material is combined with the assay sample reagent (sodium hydroxide and isopropanol), mixed by hand or vortex, and incubated at room temperature for 15 minutes. After the incubation step, $2 \mathrm{~mL}$ of the treated specimen are transferred to the cartridge and the run is initiated (Helb 2010). According to the manufacturer, Xpert MTB/RIF may be used with fresh sputum specimens, which may be either unprocessed sputum or processed sputum sediments. The sample reagent:sample volume ratio is 2:1 for unprocessed sputum and 3:1 for sputum pellets. The manufacturer does not specifically mention the use of Xpert MTB/RIF with frozen specimens (Cepheid 2009).

Xpert MTB/RIF limit of detection, (the lowest number of colony forming units per sample that can be reproducibly distinguished from negative samples with 95\% confidence) (Cepheid 2009), is five genome copies of purified DNA per reaction or 131 colony forming 
units (CFUs) per mL in M tuberculosis-spiked sputum (Helb 2010). In comparison, identification of tuberculosis bacilli by microscopic examination requires at least 10,000 bacilli per $\mathrm{mL}$ of sputum (Toman 2004a). Xpert MTB/RIF detects both live and dead bacteria (Miotto 2012).

Xpert MTB/RIF uses molecular beacon technology to detect rifampicin resistance. Molecular beacons are nucleic acid probes that recognize and report the presence or absence of the normal, rifampicin-susceptible, 'wild type' sequence of the rpoB gene of tuberculosis. Five different-coloured beacons are used, each covering a separate nucleic acid sequence within the amplified $r p o B$ gene. When a beacon binds to the matching sequence, it fluoresces or 'lights up', which indicates the presence of one of the gene sequences that is characteristic of rifampicin-susceptible tuberculo- sis. Failure of the beacon to bind or delayed binding to the matching sequence indicates potential rifampicin resistance. The number and timing of detection (when the fluorescent signal rises above a predetermined baseline cycle threshold) of positive beacons as well as results of sample processing controls allow the test to distinguish among the following results: 'No tuberculosis'; 'tuberculosis detected, rifampicin resistance detected'; 'tuberculosis detected, no rifampicin resistance detected'; and an 'invalid result' (Figure 1). A single Xpert MTB/RIF run will provide both detection of tuberculosis and detection of rifampicin resistance. One cannot deselect testing for rifampicin resistance and only run the assay for tuberculosis detection, although it is possible for the laboratory to omit results for rifampicin resistance when reporting to the healthcare provider.

Figure 1. Readout of Xpert MTB/RIF assay for a tuberculosis positive, rifampicin-susceptible specimen. Courtesy: Karin Weyer, the WHO Global TB Programme.

\section{Rifampin susceptible sample}
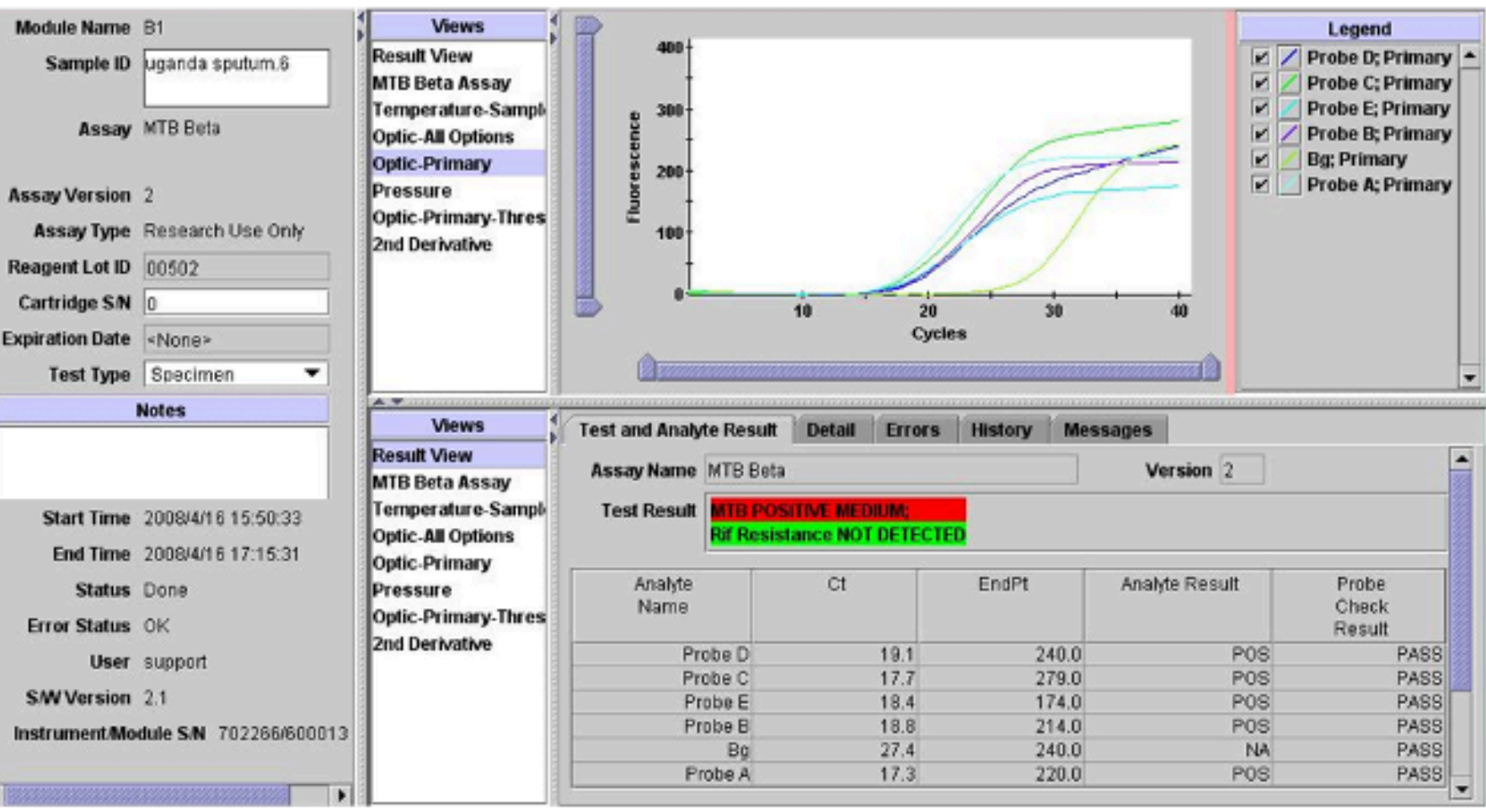

Start Time 2008j41 6 15:50:33

ad Time 20081416 17:15:31

Test Result

Rif Resistance NOT DETECTED

\begin{tabular}{|c|c|c|c|c|}
\hline $\begin{array}{l}\text { Analyte } \\
\text { Name }\end{array}$ & $\mathrm{Ct}$ & EndPt & Analyte Result & $\begin{array}{l}\text { Probe } \\
\text { Check } \\
\text { Result }\end{array}$ \\
\hline Probe 0 & 19.1 & 240.0 & POS & PA88 \\
\hline Probe C & 17.7 & 279.0 & POS & PASS \\
\hline Probe $E$ & 18.4 & 174.0 & POS & PASS \\
\hline Probe $\mathrm{E}$ & 18.8 & 214.0 & POS & PASS \\
\hline $\mathrm{Bg}$ & 27,4 & 240.0 & NA & PASS \\
\hline Probea & 17.3 & 220.0 & P0S & PASS \\
\hline
\end{tabular}

Since Xpert MTB/RIF was released, there have been four generations (G1, G2, G3, and G4) of the test involving different software and cartridge combinations. G4 contains modifications that improved determination of rifampicin resistance detection as previous Xpert MTB/RIF versions had found that some rifampicin susceptibility results were falsely resistant. In order to improve on Xpert
MTB/RIF sensitivity, Cepheid developed Xpert MTB/RIF Ultra (hereafter referred to as Xpert Ultra), a re-engineered assay that uses a newly developed cartridge but may be run on the same device after a software upgrade. Xpert Ultra incorporates two different multi-copy amplification targets and a larger DNA reaction chamber than Xpert MTB/RIF (WHO Xpert Ultra 2017). A laboratory study re- 
ported that the limit of detection using Xpert Ultra improved to 15.6 $\mathrm{CFU} / \mathrm{mL}$ of sputum compared to $112.6 \mathrm{CFU} / \mathrm{mL}$ for Xpert MTB/RIF (Chakravorty 2017). Of note, Xpert Ultra has added a new result category, 'trace call', that corresponds to the lowest bacillary burden for $M$ tuberculosis detection (WHO Xpert Ultra 2017). Although no rifampicin resistance result will be available for people with trace results, a trace positive result is sufficient to initiate anti-tuberculosis therapy in children or HIV-positive people, according to the WHO report. Other people with a trace result should have a new sputum specimen collected for Xpert Ultra testing (WHO Xpert Ultra 2017). Xpert Ultra is available for clinical use and several countries have moved from using Xpert MTB/RIF to using Xpert Ultra instead. In this Cochrane Review, we include studies that used any generation of the index tests.

\section{Clinical pathway}

Xpert MTB/RIF and Xpert Ultra are used for the diagnosis of tuberculosis and rifampicin resistance. Figure 2 shows the clinical pathway and presents the context in which the index tests might be used. The target condition is PTB. Persons to be evaluated for PTB are adults with signs or symptoms suggestive of tuberculosis, such as cough, fever, night sweats, weight loss, haemoptysis, and fatigue, or with an abnormal chest $\mathrm{x}$-ray suggestive of tuberculosis. Additionally, people who are known to have tuberculosis and are at risk for rifampicin-resistant or MDR-TB (e.g. those with a previous history of tuberculosis treatment or those who have an inadequate response to anti-tuberculosis treatment) may undergo Xpert MTB/RIF and Xpert Ultra testing to evaluate for rifampicin resistance.

Figure 2. The clinical pathway describes how people might present and the point in the pathway at which they would be considered for testing with Xpert MTB/RIF or Xpert Ultra. A person with presumptive PTB may experience cough, chest pain, the coughing up of blood, fever, night sweats, fatigue, loss of appetite, and weight loss. When she presents to a health facility, she will undergo a health examination (history and physical examination) and usually a chest X-ray. She will be tested with the index test, either Xpert MTB/RIF or Xpert Ultra, if available, as this test is recommended as the initial diagnostic test for all adults and children with signs and symptoms of tuberculosis. Abbreviations: DR-TB: drug-resistant tuberculosis; MDR-TB: multidrug-resistant tuberculosis; PTB: pulmonary tuberculosis; RIF: rifampicin; SL-LPA: second-line line probe assay; Xpert: either Xpert MTB/RIF of Xpert Ultra. Figure adapted from GLI 2018.

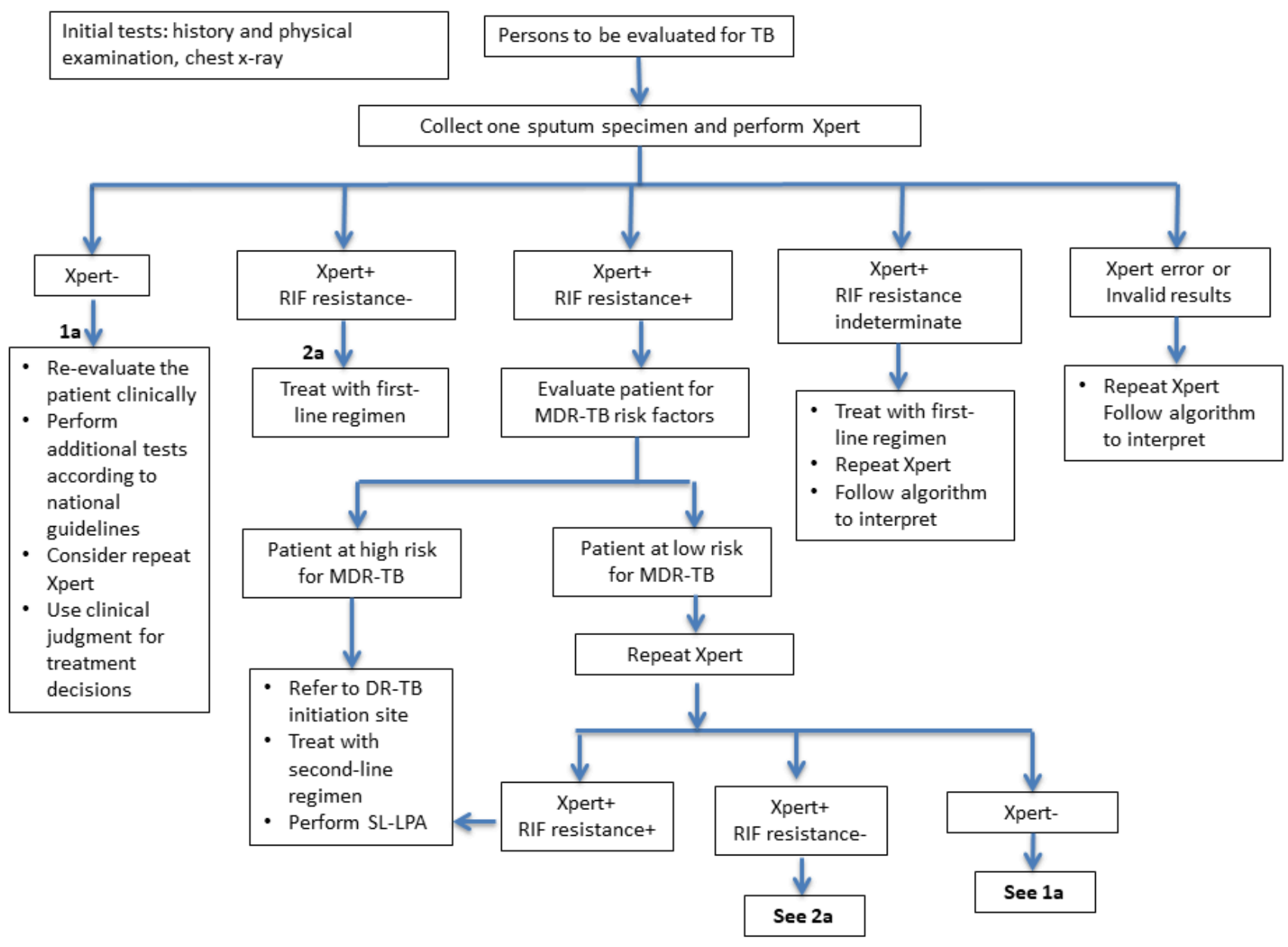

Xpert MTB/RIF and Xpert MTB/RIF Ultra for pulmonary tuberculosis and rifampicin resistance in adults (Review) 
The index test is performed as an initial test for adults with presumptive PTB or MDR-TB.

The downstream consequences of testing include the following.

- True-positive (TP): patients would benefit from rapid diagnosis and appropriate treatment.

- True-negative (TN): patients would be spared unnecessary treatment and would benefit from reassurance and pursuit of an alternative diagnosis.

- False-positive (FP): patients would probably experience anxiety and morbidity caused by additional testing, unnecessary treatment, and possible adverse events; possible stigma associated with a tuberculosis or MDR-TB diagnosis; and the chance that a false-positive result may halt further diagnostic evaluation.

- False-negative (FN): increased risk of morbidity and mortality and delayed treatment initiation; risk of ongoing tuberculosis transmission.

\section{Settings of interest}

We were interested in how the index test performed in people with presumptive PTB, who were evaluated as they would be in routine practice, most often in local hospitals or primary care centres. The index test may have the greatest impact on health when used in a setting such as a primary healthcare facility, where treatment can be started the same day as testing or as soon as possible.

It should be noted that in the original Cochrane Review, we described the setting of interest as peripheral-level laboratories based on a classification system previously in use (WHO Policy Framework 2015).

\section{Role of index test(s)}

We were interested in the following roles for testing.

\section{Xpert MTB/RIF and Xpert Ultra for detection of PTB}

Index test used as an initial test for the diagnosis of PTB.

\section{Xpert MTB/RIF and Xpert Ultra for detection of rifampicin resistance}

Index test used as an initial test for the diagnosis of rifampicin-resistant tuberculosis or MDR-TB.

As mentioned, in high MDR-TB settings the presence of rifampicin resistance alone may serve as a proxy for MDR-TB. Xpert MTB/ RIF and Xpert Ultra do not eliminate the need for subsequent culture and phenotypic drug susceptibility testing (DST), which are required to monitor treatment progress and to detect resistance to drugs other than rifampicin.

\section{Alternative test(s)}

In this section, we describe selected alternative tests for detection of PTB and rifampicin resistance. For a comprehensive review of alternative tests, we refer the reader to several excellent resources (Lewinsohn 2017; Unitaid 2017).

Smear microscopy is the examination of smears for acid-fast bacilli (tuberculosis bacteria) under a microscope. The examination may be performed by light microscopy (Ziehl-Neelsen), fluorescence microscopy, or light-emitting diode (LED) fluorescence mi- croscopy. Advantages of smear microscopy include its simplicity, low cost, speed, and high specificity in high tuberculosis burden areas. In addition, smear microscopy identifies the most infectious people with tuberculosis. Smear microscopy can be performed in basic laboratories. Drawbacks of smear microscopy include the need for specialized training and its relatively low sensitivity, 50\% to $60 \%$ on average for a direct smear (Steingart 2006b). Around 5000 to 10,000 organisms per $\mathrm{mL}$ must be present in the specimen for tuberculosis bacteria to be visible by microscopy (American Thoracic Society 2000). Although the sensitivity of microscopy can be improved by approximately $10 \%$ with fluorescence (Steingart 2006a), a large number of tuberculosis cases still go undiagnosed. Smear-negative tuberculosis is disproportionately higher in HIV-positive than in HIV-negative individuals, accounting for $24 \%$ to $61 \%$ of all pulmonary cases in people living with HIV (Getahun 2007; Perkins 2007). Microscopy cannot distinguish between drugsusceptible tuberculosis and drug-resistant tuberculosis. The WHO recommends that microscopy as the initial diagnostic test should be replaced with WHO-recommended rapid tests that can simultaneously detect tuberculosis and tuberculosis drug resistance (WHO Compendium 2018).

Mycobacterial culture is a method used to grow bacteria on nutrient-rich media. In comparison with microscopy, a positive culture requires only around 100 organisms per $\mathrm{mL}$ and therefore can detect lower numbers of tuberculosis bacteria (American Thoracic Society 2000). Additionally, culture is essential for species identification and DST. However, culture may take up to six to eight weeks and requires a highly equipped laboratory.

NAA tests are molecular systems that can detect small quantities of genetic material (DNA or RNA) from micro-organisms, such as $\mathrm{M}$ tuberculosis. The key advantage of NAA tests is that they are rapid diagnostic tests, potentially providing results in a few hours. A variety of molecular amplification methods are available, of which PCR is the most common. NAA tests are available as commercial kits and in-house tests (based on a protocol developed in a laboratory) and are used routinely in high-income countries for tuberculosis detection. In-house PCR is widely used in low-income countries because these tests are less expensive than commercial kits. However, inhouse PCR is known to produce highly inconsistent results (Flores 2005).

Alternative molecular methods for DST include the commercial line probe assays, GenoType MTBDRplus assay (MTBDRplus, Hain LifeScience, Nehren, Germany), and the Nipro NTM+MDRTB detection kit 2 (Nipro, Tokyo, Japan), which detect the presence of mutations associated with drug resistance to isoniazid and rifampicin (Nathavitharana 2017). MTBDRplus is the most widely studied line probe assay. Advantages of line probe assays are that they can provide a result for detection of tuberculosis and drug resistance in one to two days. Drawbacks are that line probe assays are expensive and need to be used in intermediate and central laboratories (Unitaid 2017). The WHO recommends that for persons with a sputum smear-positive specimen or a cultured tuberculosis isolate, commercial molecular line probe assays may be used as the initial test instead of phenotypic culture-based DST to detect resistance to rifampicin and isoniazid (conditional recommendation, moderate certainty in the evidence for the test's accuracy) (WHO LPA 2016). Other molecular assays for detection of tuberculosis and resistance 
to rifampicin and isoniazid along with instruments are in development (Walzl 2018).

Alere Determine ${ }^{\mathrm{TM}}$ TB LAM Ag (AlereLAM) Alere Inc, (Waltham, USA) is a commercially available point-of-care test for tuberculosis disease (PTB and extrapulmonary tuberculosis). The test detects lipoarabinomannan (LAM), a component of the bacterial cell wall, which is present in the urine of some people with tuberculosis. AlereLAM is performed by placing urine on one end of a test strip, with results appearing as a band on the strip if tuberculosis is present. The test is simple, requires no special equipment, and shows results in 25 minutes (Shah 2016). Of note, the presence of LAM in the urine of HIV-positive adults undergoing treatment for tuberculosis has been found to be associated with increased risk of mortality (Gupta-Wright 2018). In randomized trials, use of Alere LAM in HIV-positive inpatients has been shown to reduce mortality (Gupta-Wright 2018; Peter 2016). Based in part on evidence from a Cochrane Review, Shah 2016, the WHO recommends that AlereLAM should be used to assist in the diagnosis of tuberculosis in adult inpatients, specifically "people living with HIV who have signs or symptoms of tuberculosis and a CD4 cell count less than or equal to 100 cells $/ \mu \mathrm{L}$, and people living with HIV who are 'seriously ill' regardless of CD4 count or if the CD4 count is unknown. This recommendation also applies to HIV-positive children with signs and symptoms of tuberculosis (pulmonary or extrapulmonary, or both) based on the generalisation of data from adults while acknowledging very limited data and concern regarding low specificity of the AlereLAM assay in children" (WHO LAM 2015). The WHO does not recommend AlereLAM for tuberculosis screening or diagnosis of active tuberculosis disease in most population groups (WHO LAM 2015).

Fujifilm SILVAMP TB LAM (FuijiLAM, co-developed by FIND, Geneva, Switzerland and Fujifilm, Tokyo, Japan) is a new, urine-based, point-of-care test for tuberculosis diagnosis in people living with HIV. Using stored (biobanked) urine specimens from hospitalized people in South Africa, FujiLAM was found to have superior sensitivity, $70.4 \%$ (95\% $\mathrm{Cl} 53.0 \%$ to $83.1 \%$ ) compared to AlereLAM sensitivity of $42.3 \%$ (31.7\% to $51.8 \%$ ) (Broger 2018). At the time of this writing, a call was open for prospective clinical trials of FuijiLAM to generate data for an updated WHO policy review.

\section{Rationale}

Xpert MTB/RIF and Xpert Ultra provide obvious benefits for patients (earlier diagnosis and the opportunity to begin earlier, appropriate treatment) and for public health (opportunities to interrupt tuberculosis transmission), especially in high tuberculosis burden countries.

Since 2010, the WHO has recommended the use of Xpert MTB/RIF as the preferred initial diagnostic test for people thought to have MDR-TB or HIV-associated tuberculosis (strong recommendation, moderate-certainty evidence) (WHO Policy Xpert MTB/RIF 2011). In 2013, the WHO expanded the recommendations, stating that Xpert MTB/RIF may be used rather than conventional microscopy and culture as the initial diagnostic test in all adults suspected of having tuberculosis (conditional recommendation acknowledging resource implications, high-quality evidence) (WHO Xpert MTB/ RIF Policy Update 2013). In addition, the WHO recommended that following an Xpert MTB/RIF test that demonstrates rifampicin resistance, subsequent drug susceptibility testing (e.g. using a line probe assay to second-line drugs) remains essential to detect re- sistance to drugs other than rifampicin (WHO Xpert MTB/RIF Policy Update 2013). In 2017, based on a non-inferiority analysis of Xpert Ultra compared with Xpert MTB/RIF, the WHO stated that recommendations on the use of Xpert MTB/RIF also apply to the use of Xpert Ultra as the initial diagnostic test for all adults and children with signs and symptoms of tuberculosis (WHO Xpert Ultra 2017). We performed this Cochrane Review to inform an updated WHO policy review on the use of Xpert MTB/RIF and Xpert Ultra.

\section{O B JECT IVES}

\section{Primary objectives}

To determine the diagnostic accuracy of Xpert MTB/RIF and Xpert Ultra for tuberculosis in adults with presumptive PTB, and for rifampicin resistance in adults with presumptive rifampicin-resistant tuberculosis or MDR-TB.

\section{Secondary objectives}

- To compare the diagnostic accuracy of Xpert MTB/RIF and Xpert Ultra.

- To investigate potential sources of heterogeneity in test accuracy. For detection of PTB, covariates were smear status; HIV status; history of tuberculosis; the setting that ran the test; tuberculosis burden; TB/HIV burden; and prevalence of PTB in the studies. For detection of rifampicin resistance, covariates were MDRTB burden and prevalence of rifampicin resistance in the studies.

\section{METHODS}

\section{Criteria for considering studies for this review}

\section{Types of studies}

We include cross-sectional studies and cohort studies that assessed the diagnostic accuracy of the index test(s) for both PTB and rifampicin resistance, PTB alone, or rifampicin resistance alone. We also include randomized controlled trials (RCTs) that evaluated the use of the index(s) test on patient health outcomes, but that also reported sensitivity and specificity. Although the study design was a randomized trial for the purpose of determining the impact of the test on participant outcomes, the study design was a cross-sectional study for the purpose of determining the diagnostic accuracy of the index tests in this review. We used abstracts to identify published studies and included these publications when they met our inclusion criteria. We only included studies that reported data comparing the index test(s) to an acceptable reference standard from which we could extract true positive (TP), true negative (TN), false positive (FP), and false negative (FN) values. The index tests could be assessed alone or together with other tests.

We included studies that evaluated the index tests in HIV-positive people irrespective of tuberculosis symptoms, for instance HIVpositive people being assessed for antiretroviral therapy, as in the study by Lawn 2011. We included these studies for the following reasons: the risk of developing tuberculosis is much higher in people living with HIV, estimated to be 20 to 37 times higher in HIV-positive individuals than in HIV-negative individuals (Getahun 2010); signs and symptoms of tuberculosis in people living with HIV vary, which makes it challenging to determine when to consider a diagnosis of tuberculosis; and many HIV-positive people in low-income countries develop tuberculosis as the first manifestation of AIDS. 
We excluded case reports and studies with a case-control design, the latter because these types of studies are prone to bias, in particular, studies enrolling participants with severe disease and healthy participants without disease. We excluded studies of the index tests in people with diabetes but without tuberculosis symptoms, and studies designed to find people with active tuberculosis in community settings. We excluded drug resistance surveys.

\section{Participants}

We included studies that enrolled adults, aged 15 years or older, with presumptive PTB, rifampicin-resistant tuberculosis, or MDRTB. For tuberculosis detection, we were interested in people who were not currently on tuberculosis treatment or those on treatment for less than seven days. Tuberculosis treatment might interfere with the confirmation of tuberculosis on culture (the reference standard for this review). If we could not tell the treatment status of the participants, we contacted primary study authors for this information. For rifampicin resistance detection, we were interested in people at high risk for MDR-TB and we therefore included participants who had received previous treatment, participants who were receiving tuberculosis treatment because they had not converted their sputum from positive to negative, and contacts with participants with known drug-resistant disease, as described in Boehme 2010.

We included studies that assessed the diagnostic accuracy of Xpert MTB/RIF (Xpert MTB/RIF) and Xpert MTB/RIF Ultra (Xpert Ultra) using sputum and other respiratory specimens, such as fluid obtained from bronchial alveolar lavage and tracheal aspiration, consistent with the intended use of the manufacturer (Cepheid 2009), and studies from all types of health facilities and all laboratory levels (peripheral, intermediate, and central) from all countries. Unlike the original Cochrane Reviews, for this review update if a study included both adults and children and we could not disaggregate results for adults alone, we excluded the study. We also excluded studies where the age of participants was unknown.

\section{Index tests}

The index tests were Xpert MTB/RIF and Xpert Ultra.

Index test results are automatically generated (i.e. there is a single threshold), and the user is provided with a printable test result as follows.

- MTB (M tuberculosis) DETECTED; Rif (rifampicin) resistance DETECTED.

- MTB DETECTED; Rif resistance NOT DETECTED.

- MTB detected; Rif resistance INDETERMINATE.

- MTB NOT DETECTED.

- INVALID (the presence or absence of MTB cannot be determined).

- ERROR (the presence or absence of MTB cannot be determined).

- NO RESULT (the presence or absence of MTB cannot be determined).

Xpert Ultra incorporates a semi-quantitative classification for results: trace, very low, low, moderate, and high. 'Trace' corresponds to the lowest bacterial burden for detection of $M$ tuberculosis (Chakravorty 2017). We considered a trace result to mean MTB ( $M$ tuberculosis) DETECTED. However, no rifampicin-resistance result was available for participants with trace results (WHO Xpert Ultra 2017).

\section{Target conditions}

The target conditions were active PTB and rifampicin resistance.

\section{Reference standards}

For tuberculosis, acceptable reference standards used solid media (Löwenstein-Jensen, Middlebrook $7 \mathrm{H} 10$ or $7 \mathrm{H} 11$, or Ogawa media) or a commercial liquid culture system, (such as BACTEC ${ }^{\text {TM }}$ 460TB System or BACTEC ${ }^{\text {TM }}$ MGIT ${ }^{\text {TM }} 960$ Mycobacterial Detection System, BD, USA; BacT/ALERT System, bioMérieux, France; or VersaTREK Mycobacteria Detection \& Susceptibility, Thermo Fisher Scientific, USA).

For rifampicin resistance, the reference standards were phenotypic culture-based DST methods recommended by the WHO (WHO Policy DST 2008). Acceptable methods were the proportion method performed on solid media (such as Löwenstein-Jensen, Middlebrook $7 \mathrm{H} 10$ or $7 \mathrm{H} 11$, or Ogawa media), use of a commercial liquid culture system, such as MGIT ${ }^{\mathrm{TM}} 960$ Mycobacterial Detection System, BD, USA, or both. For this review update, we also included MTBDRplus, a WHO-recommended test (WHO LPA 2016).

\section{Search methods for identification of studies}

We tried to identify all relevant studies regardless of language or publication status (published, unpublished, in press, and ongoing).

\section{Electronic searches}

We searched the following databases up to 18 January 2018, using the search terms and strategy described in Appendix 1 :

- Cochrane Infectious Diseases Group Specialized Register;

- MEDLINE (OVID, from 1966);

- Embase (OVID, from 1974);

- Science Citation Index - Expanded (from 1900), Conference Proceedings Citation Index - Science (CPCI-S, from 1990), and BIOSIS Previews (from 1926); all three from the Web of Science;

- Scopus (Elsevier, from 1970);

- Latin American Caribbean Health Sciences Literature (LILACS) (BIREME, from 1982).

We also searched ClinicalTrials.gov, the WHO International Clinical Trials Registry Platform (ICTRP; www.who.int/trialsearch), and the International Standard Randomized Controlled Trials Number (ISRCTN) registry (www.isrctn.com/) for trials in progress, and ProQuest Dissertations \& Theses A\&I (1990 to 7 August 2017) for dissertations. On 11 October 2018, we performed an additional search, specifically for studies that evaluated Xpert Ultra.

To identify other systematic reviews and meta-analyses, we performed an additional search on 26 March 2018 in MEDLINE (PubMed), Embase (OVID) and the Cochrane Library, Issue 7 2018, applying filters for systematic reviews (www.sign.ac.uk/search-filters.html) to search terms for Xpert and tuberculosis.

\section{Searching other resources}

We reviewed reference lists of included articles and any relevant review articles identified through the above methods. We also contacted researchers at FIND, the WHO Global TB Programme, and 
other experts in the field of tuberculosis diagnostics for information on ongoing and unpublished studies.

\section{Data collection and analysis}

\section{Selection of studies}

We used Covidence to manage the selection of studies (Covidence 2017). Working in pairs, four review authors independently scrutinized titles and abstracts identified from literature searching to identify potentially eligible studies. We retrieved the article of any citation identified by any review author for full-text review. Then, again working in pairs, four review authors independently assessed articles for inclusion using predefined inclusion and exclusion criteria, and resolved any discrepancies by discussion among all review authors. We recorded all studies excluded after full-text assessment and their reasons for exclusion in the Characteristics of excluded studies table. We illustrated the study selection process in a PRISMA diagram. We included search results from the original review and re-evaluated previously included studies to determine if the studies met the refined inclusion criteria.

In the 2014 Cochrane Review (Steingart 2014), for the multicentre studies Boehme 2010 (five study centres) and Boehme 2011 (six study centres), we entered data separately for each study centre. We did not repeat this for this updated review and hence we count Boehme 2010 and Boehme 2011 each as one study and present the two-by-two data for the total population in each study. Appendix 2 presents the data by individual study centre.

\section{Data extraction and management}

We extracted data on the following characteristics.

- Author, publication year, study design, country where study was located, level of laboratory services, setting (outpatient, inpatient, or both outpatient and inpatient) and whether the test was run at point of care.

- Population characteristics: age, gender, smear status, HIV status.

- Index test(s), Xpert MTB/RIF or Xpert Ultra.

- Reference standard.

- Condition of the specimen (fresh or frozen).

- Quality Assessment of Studies of Diagnostic Accuracy - Revised (QUADAS-2) items (Whiting 2011).

- Number of TP, FP, FN, and TN (i.e. true positives, false positives, false negatives, and true negatives, with respect to culture).

- Number of uninterpretable results for detection of PTB.

- Number of indeterminate results for detection of rifampicin resistance.

We classified country income status as either low- and middle-income or high-income, according to the World Bank List of Economies (World Bank 2017). In addition, we classified 'country' as being high burden or not high burden for tuberculosis, TB/HIV, or MDR-TB, according to the post-2015 era classification by the WHO (WHO Global TB Report 2018). A country could be classified as high burden for one, two, or all three of the high burden categories.

We classified the level of laboratory that ran the index tests as being one of three service levels: peripheral, intermediate, or central (GLI 2015). Peripheral laboratories may perform Xpert MTB/RIF or Xpert Ultra testing, but typically perform only smear microscopy, and will refer specimens or people in need of further tests, such as rapid molecular testing, culture, or DST, to a higher-level laboratory. Intermediate laboratories typically perform tests such as microscopy, rapid molecular tests, culture on solid media and line probe assays on sputum. Central laboratories run intermediate laboratory tests, as well as culture on liquid media and DST on solid or liquid media to detect resistance to first- and second-line anti-tuberculosis drugs, line probe assays on positive cultures, and rapid speciation tests (GLI 2015).

Whenever possible, we extracted TP, FP, FN, and TN values based on one Xpert MTB/RIF or Xpert Ultra result for one specimen provided by one participant. However, in some of the studies, the number of specimens (and index test results) exceeded the number of participants, suggesting that a single participant may have provided multiple specimens. We therefore compared pooled sensitivity and specificity for tuberculosis detection in all studies with pooled sensitivity and specificity in the subset of studies that provided one index test result based on one specimen provided by one participant (see Sensitivity analyses).

Concerning the condition of the specimen, although the manufacturer recommends use of fresh specimens, we were aware that several studies had been conducted using frozen specimens so we extracted this information as well. We investigated the influence of condition of specimen in a sensitivity analysis.

Concerning the definition of smear positivity, as most included studies performed the index tests in intermediate-level or central-level laboratories, we assumed these studies adhered to the revised definition of a new sputum smear-positive PTB case based on the presence of at least one acid-fast bacillus in at least one sputum sample in countries with a well-functioning external quality assurance system (WHO Policy Smear-positive TB Case 2007).

We developed a standardized data extraction form and piloted the form with 10 studies. Based upon the pilot, we finalized the form. Four review authors working in pairs independently extracted data from each study using the final form. We contacted study authors for missing data and clarifications and managed all data with REDCap (Harris 2009). The final data extraction form is in Appendix 3. With regard to the use of REDCap, the content in this review is solely the responsibility of the authors and does not necessarily represent the official views of the National Institutes of Health.

We followed Cochrane policy, which states that "authors of primary studies will not extract data from their own study or studies. Instead, another author will extract these data, and check the interpretation against the study report and any available study registration details or protocol".

\section{Assessment of methodological quality}

We used the QUADAS-2 tool, tailored to this review, to assess the quality of the included studies (Appendix 4) (Whiting 2011). QUADAS-2 consists of four domains: patient selection, index test, reference standard, and flow and timing. We assessed all domains for the potential for risks of bias and the first three domains for concerns regarding applicability. Four review authors, working independently in pairs, completed QUADAS-2 and resolved disagreements through discussion. We present the results of this quality assessment in text, tables, and graphs. 


\section{Statistical analysis and data synthesis}

We performed descriptive analyses for the results of the included studies using Stata 15 (Stata 2017). We determined sensitivity and specificity estimates and $95 \%$ confidence intervals (Cls) for individual studies and generated forest plots using Review Manager 2014. Whenever possible, we included nontuberculous mycobacteria (NTM) as non-tuberculosis for specificity determinations. We chose to use data that were not subject to discrepant analyses (unresolved data), since resolved data after discrepant analyses are a potential for risk of bias (Hadgu 2005).

We carried out meta-analyses to estimate the pooled sensitivity and specificity of the index tests separately for tuberculosis detection and rifampicin resistance detection. When possible, we determined pooled estimates using an adaptation of the bivariate random-effects model of Reitsma 2005, which uses the exact binomial likelihood for the observed proportions (Chu 2006). We accounted for the hierarchical structure of two multicentre studies for which individual centre data were available by adding a random effect for each centre (Boehme 2010; Boehme 2011). The bivariate random-effects approach allowed us to calculate the pooled estimates of sensitivity and specificity while dealing with potential sources of variation caused by (1) imprecision of sensitivity and specificity estimates within individual studies; (2) correlation between sensitivity and specificity across studies; and (3) variation in sensitivity and specificity between studies. For Xpert MTB/RIF and Xpert Ultra for PTB detection among smear-positive individuals (described below), we performed a univariate analysis.

For the primary analysis for Xpert MTB/RIF or Xpert Ultra for tuberculosis detection, we first estimated accuracy using all studies meeting our inclusion criteria and then using only the subset of studies where participants were unselected. In the latter analysis, we excluded studies that preselected participants based on prior microscopy testing or primarily included participants with a history of previous tuberculosis treatment.

\section{Rifampicin resistance detection}

For analysis of Xpert MTB/RIF or Xpert Ultra accuracy for detection of rifampicin resistance, we included participants who (1) were culture-positive; (2) had a valid phenotypic DST (or MTBDRplus) result (3) were Xpert MTB/RIF (or Xpert Ultra) tuberculosis-positive; and (4) had a valid Xpert MTB/RIF (or Xpert Ultra) Rif result.

- Sensitivity $=$ Xpert MTB/RIF (or Xpert Ultra) Rif resistant/DST Rif resistant.

- Specificity = Xpert MTB/RIF (or Xpert Ultra) Rif susceptible/DST Rif susceptible.

For rifampicin resistance detection, we performed bivariate metaanalyses to determine sensitivity and specificity estimates.

\section{Comparison of Xpert MTB/RIF and Xpert Ultra}

We intended to perform meta-analyses of the accuracy of Xpert MTB/RIF and Xpert Ultra by first including all studies with relevant data, i.e. indirect comparisons, and then by restricting the analyses to studies that made comparisons between Xpert MTB/RIF and Xpert Ultra in the same participants, i.e. direct comparisons (Takwoingi 2013). However, we identified only one study using Xpert Ultra and this study compared Xpert MTB/RIF and Xpert Ultra on the same participant specimens (Dorman 2018). As in the primary analysis in Dorman 2018, Xpert Ultra trace calls in this review were considered to be positive for the detection of $M$ tuberculosis.

We estimated all models using a Bayesian approach with low-information prior distributions using OpenBUGS software (Version 3.2.3) (Lunn 2009), along with $\mathrm{R}$ (Version 3.3.2) (R Core Team 2016). Under the Bayesian approach, all unknown parameters must be provided a prior distribution that defines the range of possible values of the parameter and the likelihood of each of those values based on information external to the data. In order to let the observed data determine the final results, we chose to use low-information prior distributions over the pooled sensitivity and specificity parameters and their between-study standard deviation parameters. We summarize the model we used in the Statistical Appendix together with the OpenBUGS programme used to implement it (Appendix 5). It is known that meta-analysis models can be sensitive to the choice of prior distributions over between-study standard deviation parameters. We therefore carried out sensitivity analyses and considered alternative prior distributions that are less informative, allowing a wider range of possible values. To study the sensitivity of all results to the choice of prior distributions, we considered alternative prior distributions that were less informative, allowing a wider range of possible values. We noted no appreciable change in pooled accuracy parameters but, as expected, found that the posterior credible intervals and prediction intervals were slightly wider. Information from the prior distribution is combined with the likelihood of the observed data in accordance with Bayes theorem to obtain a posterior distribution for each unknown parameter (Appendix 6).

Using a sample from the posterior distribution, we can obtain various descriptive statistics of interest. We estimated the median pooled sensitivity and specificity and their $95 \%$ credible intervals (Crls). The median or the $50 \%$ quantile is the value below which lies $50 \%$ of the posterior sample. We reported the median because the posterior distributions of some parameters may be skewed and the median would be considered a better point estimate of the unknown parameter than the mean in such cases. The $95 \% \mathrm{Crl}$ is the Bayesian equivalent of the classical (frequentist) $95 \% \mathrm{Cl}$. (We have indicated $95 \% \mathrm{Cl}$ for individual study estimates and $95 \% \mathrm{Crl}$ for pooled study estimates, as appropriate). The $95 \% \mathrm{Crl}$ may be interpreted as an interval that has a $95 \%$ probability of capturing the true value of the unknown parameter, given the observed data and the prior information.

We also estimated the 'predicted' sensitivity and specificity in a future study together with their $95 \%$ Crls. The predicted estimate is our best guess for the estimate in a future study and is the same as the pooled estimate. The Crls, however, may be different. These values are derived from the predicted region typically reported in a bivariate meta-analysis plot. If there is no heterogeneity at all between studies, the $\mathrm{Cl}$ (or $\mathrm{Crl}$ ) around the predicted estimate will be the same as the $\mathrm{Cl}$ around the pooled estimate. On the other hand, if there is considerable heterogeneity between studies, the $\mathrm{Cl}$ around the predicted estimate will be much wider than the $\mathrm{Cl}$ around the pooled estimate. We generated the plots using $R$ (version 3.3.2) ( $R$ Core Team 2016).

\section{Approach to uninterpretable index test results}

The index tests report an uninterpretable test result for unexpected results with any of the internal control measures of the assay. The uninterpretable rate for detection of PTB was the number of 
tests classified as 'invalid', 'error', or 'no result' divided by the total number of index tests performed. The uninterpretable rate for detection of rifampicin resistance (referred to as indeterminate rate) was the number of tests classified as 'MTB detected; Rif resistance INDETERMINATE' divided by the total number of index test-positive results. As we found very few uninterpretable results reported, we excluded these results from the quantitative analysis. We used a Bayesian hierarchical model for a single proportion to estimate the pooled proportion of uninterpretable index test results.

\section{Investigations of heterogeneity \\ Detection of PTB}

\section{Effect of smear status and HIV status}

We investigated heterogeneity by performing subgroup analyses to determine sensitivity and specificity estimates for participants grouped by smear or HIV status. We analysed the data in two ways: 1) we performed meta-analyses where we included all studies with available data, and 2) we performed meta-analyses restricting the analysis to studies that provided data for both smear-positive and smear-negative individuals (or both HIV-negative and HIV-positive individuals) within the same study. In the latter comparison, we hoped to achieve a similar distribution of other participant characteristics and manner of test execution in the subgroups.

For smear-positive tuberculosis, we performed a univariate analysis for sensitivity. We did this because in many studies the value for true negatives was zero (tuberculosis was not detected when defined by a positive culture), and we considered all participants to be true positives. It has been observed among individuals with presumptive tuberculosis that when a sputum specimen is found to be positive by smear microscopy, the probability of a culture being negative is low (Toman 2004b).

\section{Effect of other covariates}

To study the impact of additional covariates of interest, we performed subgroup analyses with the following covariates.

\section{PTB detection}

- High tuberculosis burden, yes or no.

- High TB/HIV burden, yes or no.

- Percentage of participants with a history of tuberculosis, greater than the median value versus less than or equal to the median value.

- Setting that ran the test, point of care or peripheral setting versus intermediate or central laboratory.

- Prevalence of PTB in the studies, greater than the median value versus less than or equal to the median value.

All the aforementioned covariates were categorical, study-level covariates. For these analyses, we restricted the studies to those that included unselected participants, i.e. we excluded studies that preselected participants on the basis of a prior smear microscopy result or primarily included participants with a history of previous tuberculosis treatment.

\section{Detection of rifampicin resistance}

For rifampicin resistance detection, we performed subgroup analyses with the following covariates.
- High MDR-TB burden, yes or no.

- Studies involving participants who had received previous tuberculosis treatment, yes or no.

- Prevalence of rifampicin resistance in the studies, greater than the median value versus less than or equal to the median value.

All the aforementioned covariates were categorical, study-level covariates.

\section{Sensitivity analyses}

For detection of PTB, we performed sensitivity analyses by limiting inclusion in the meta-analysis based on the following criteria.

- Studies that explicitly represented the use of the index tests for the diagnosis of individuals with signs and symptoms of tuberculosis (presumptive tuberculosis). We excluded studies that involved HIV-positive participants irrespective of tuberculosis symptoms.

- Studies where a single specimen yielded a single Xpert MTB/RIF result for a given participant. We excluded studies that included more specimens than participants.

- Studies that included only untreated participants. We excluded studies that did not explicitly state they included only untreated participants.

- Studies that used liquid culture as the reference standard.

- Studies where a consecutive or random sample of participants were enrolled.

- Studies where the reference standard was blinded.

- Studies that only used fresh specimens.

- Studies that accounted for all participants in the analysis. We excluded studies where we answered 'no' or 'unclear' to the QUADAS-2 Flow and Timing signalling question: Were all patients included in the analysis?

In addition, in order to assess the influence of two large multicentre manufacturer-supported studies on the summary estimates, we performed an analysis excluding these studies (Boehme 2010; Boehme 2011).

For the sensitivity analyses, we restricted the studies to those that included unselected participants; i.e. we excluded studies that preselected participants on the basis of a prior smear microscopy result or previous tuberculosis treatment.

\section{Assessment of reporting bias}

We chose not to carry out formal assessment of publication bias using methods such as funnel plots or regression tests, because such techniques have not been helpful for diagnostic test accuracy studies (Macaskill 2010). However, Xpert MTB/RIF and Xpert Ultra are produced by only one manufacturer and, as tests for which there has been considerable attention and scrutiny, we believe reporting bias was minimal.

\section{Other analyses}

\section{Nontuberculous mycobacteria (NTM)}

NTM, such as $M$ avium complex and $M$ intracellulare, comprise a multi-species group of human pathogens that are ubiquitous in water and soil. NTMs can cause severe pulmonary and other diseases that share clinical signs with tuberculosis but are treated differently. People living with HIV with severe immunosuppression are par- 
ticularly vulnerable to infections caused by NTM (Gopinath 2010). We summarized separately data for NTM by determining the percent of false-positive Xpert MTB/RIF results (data were only reported for Xpert MTB/RIF) in samples that grew NTMs (see Results: Other analyses: NTM).

\section{Assessment of certainty of the evidence}

Four review authors assessed the certainty of the evidence (also called quality of the evidence) using the GRADE approach (Balshem 2011; Schünemann 2008; Schünemann 2016), and GRADEpro Guideline Development Tool (GDT) software (GRADEpro GDT 2015). In the context of a systematic review, ratings of the certainty of the evidence reflect the extent of our confidence that the estimates of effect (including test accuracy and associations) are correct. As recommended, we rated the certainty of the evidence as either high (not downgraded), moderate (downgraded by one level), low (downgraded by two levels), or very low (downgraded by more than two levels) for five domains: risk of bias, indirectness, inconsistency, imprecision, and publication bias.

For each outcome, we considered the certainty of the evidence to begin as high when high-quality observational studies (cross-sectional or cohort studies) enrolled participants with diagnostic uncertainty. If we had a reason for downgrading, we used our judgement to classify the reason as serious (downgraded by one level) or very serious (downgraded by two levels). We summarized this information in the 'Summary of findings' tables (Summary of findings 1 ; Summary of findings 2).

We applied GRADE in the following ways.

- Risk of bias: we used QUADAS-2 to assess risk of bias.

- Indirectness: we used QUADAS-2 for concerns of applicability and looked for important differences between the populations studied (for example, the spectrum of disease), the setting, index test, and outcomes, and asked whether differences were sufficient to lower certainty in results.

- Inconsistency: GRADE recommends downgrading for unexplained inconsistency in sensitivity and specificity estimates. We carried out prespecified analyses to investigate potential sources of heterogeneity and did not downgrade when we believed we could explain inconsistency in the accuracy estimates.

- Imprecision: we considered a precise estimate to be one that would allow a clinically meaningful decision. We considered the width of the $\mathrm{Crl}$ and asked ourselves, 'Would we make a different decision if the lower or upper boundary of the Crl represented the truth?'. In addition, we worked out projected ranges for TP, FN, TN, and FP for a given prevalence of tuberculosis and made judgements on imprecision from these calculations. We also considered whether the number of participants included in the analysis was less than the number generated by a conventional sample size calculation for a single adequately-powered study.

- Publication bias: we rated publication bias as undetected (not serious) because of the comprehensiveness of the literature search and following extensive outreach to tuberculosis researchers to identify studies. As we included a large number of studies, we thought that had we missed several small studies, the results would probably not be different.

\section{RES U LTS}

\section{Results of the search}

We identified 95 unique studies, integrating 77 new studies since publication of the Cochrane Review (Steingart 2014). All studies but one (Huang 2015 in Chinese) were written in English. For PTB detection, rifampicin resistance detection, or both PTB and rifampicin resistance detection, all 95 studies evaluated Xpert MTB/RIF (Xpert MTB/RIF) and one study compared Xpert MTB/RIF and Xpert MTB/ RIF Ultra (Xpert Ultra) (Dorman 2018). Of the total 86 studies for PTB detection, 48 studies evaluated the test for detection of both PTB and rifampicin resistance and 38 studies for PTB alone. Of the total 57 studies for rifampicin resistance detection, nine studies evaluated the test for rifampicin resistance alone. Figure 3 shows the flow of studies in the review. We recorded the excluded studies, including those listed in the previous Cochrane Review (Steingart 2014), and the reasons for their exclusion in the Characteristics of excluded studies table. 
Figure 3. Flow diagram of studies in the review. To identify other systematic reviews, we performed an additional literature search on 26 March 2018 (Table 5).

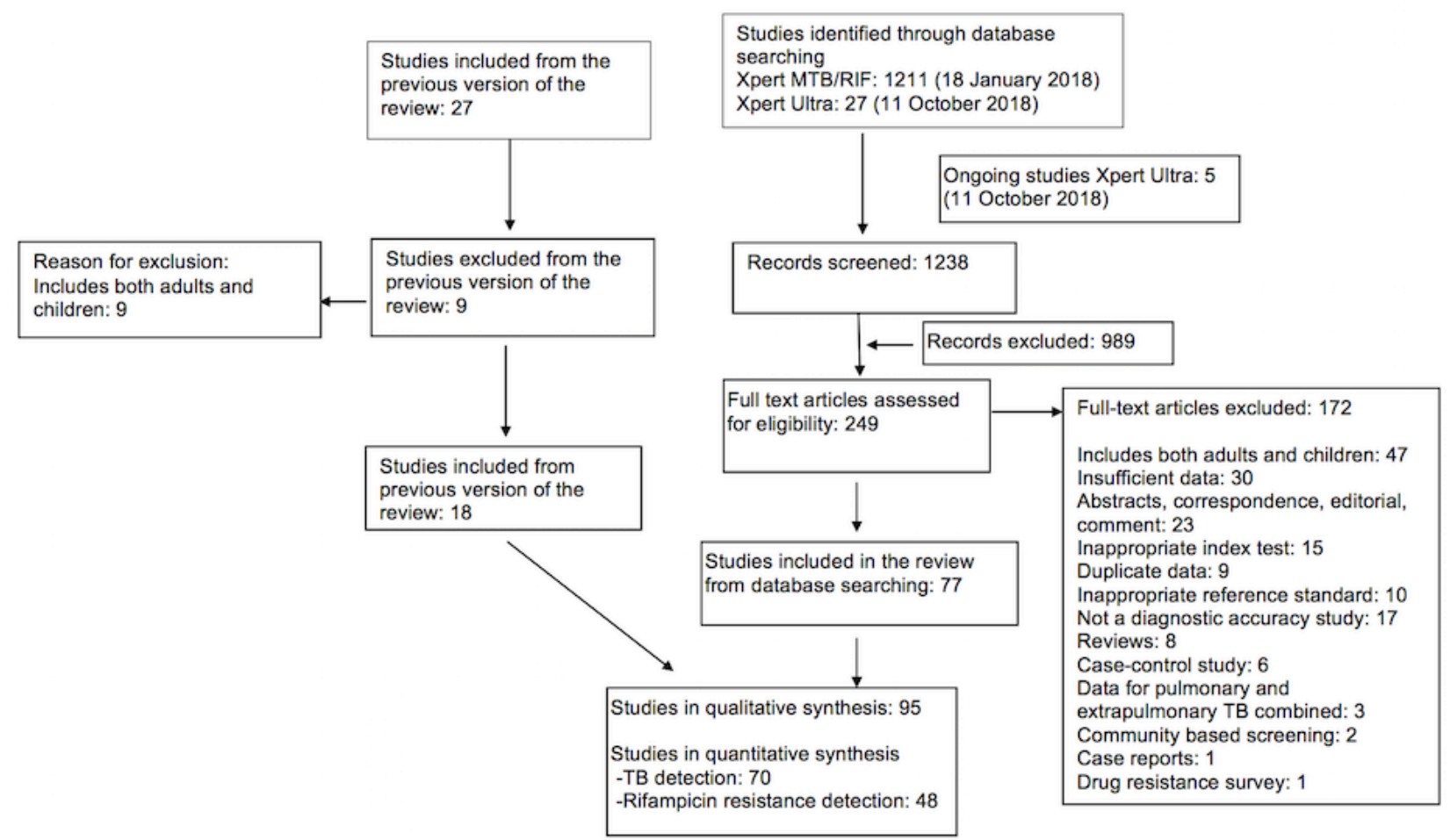

\section{Methodological quality of included studies}

Studies evaluating Xpert MTB/RIF and Xpert Ultra for detection of PTB

Figure 4, Figure 5, and Figure 6 show risk of bias and applicability concerns for 86 studies evaluating Xpert MTB/RIF and Xpert Ultra for tuberculosis detection. 
Figure 4. Risk of bias and applicability concerns graph for pulmonary tuberculosis detection: review authors' judgements about each domain presented as percentages across included studies.

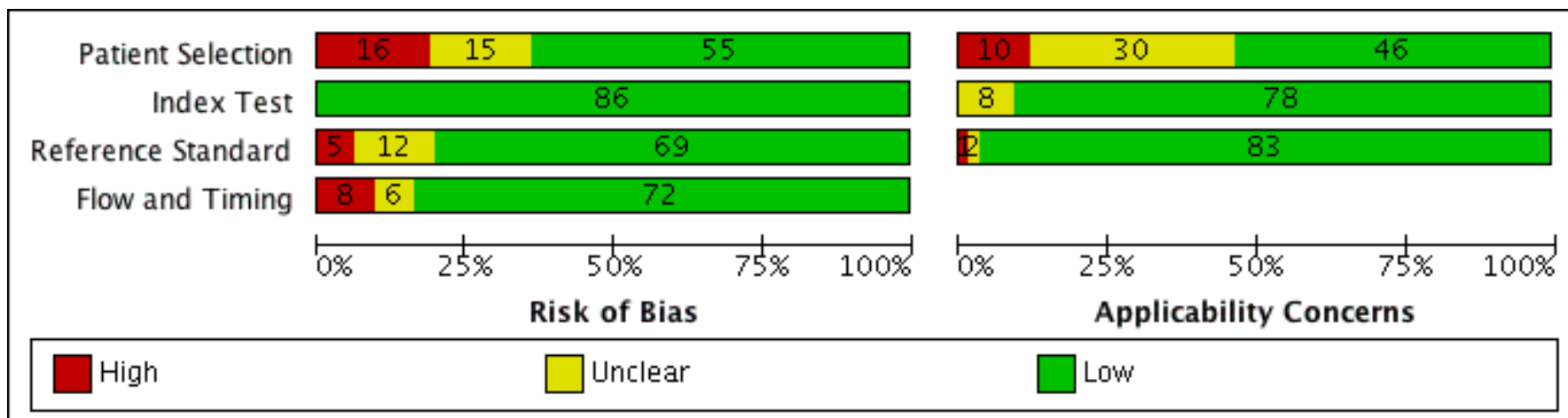


Figure 5. Risk of bias and applicability concerns summary for pulmonary tuberculosis detection: review authors' judgements about each domain for each included study, studies A through $\mathrm{K}$.

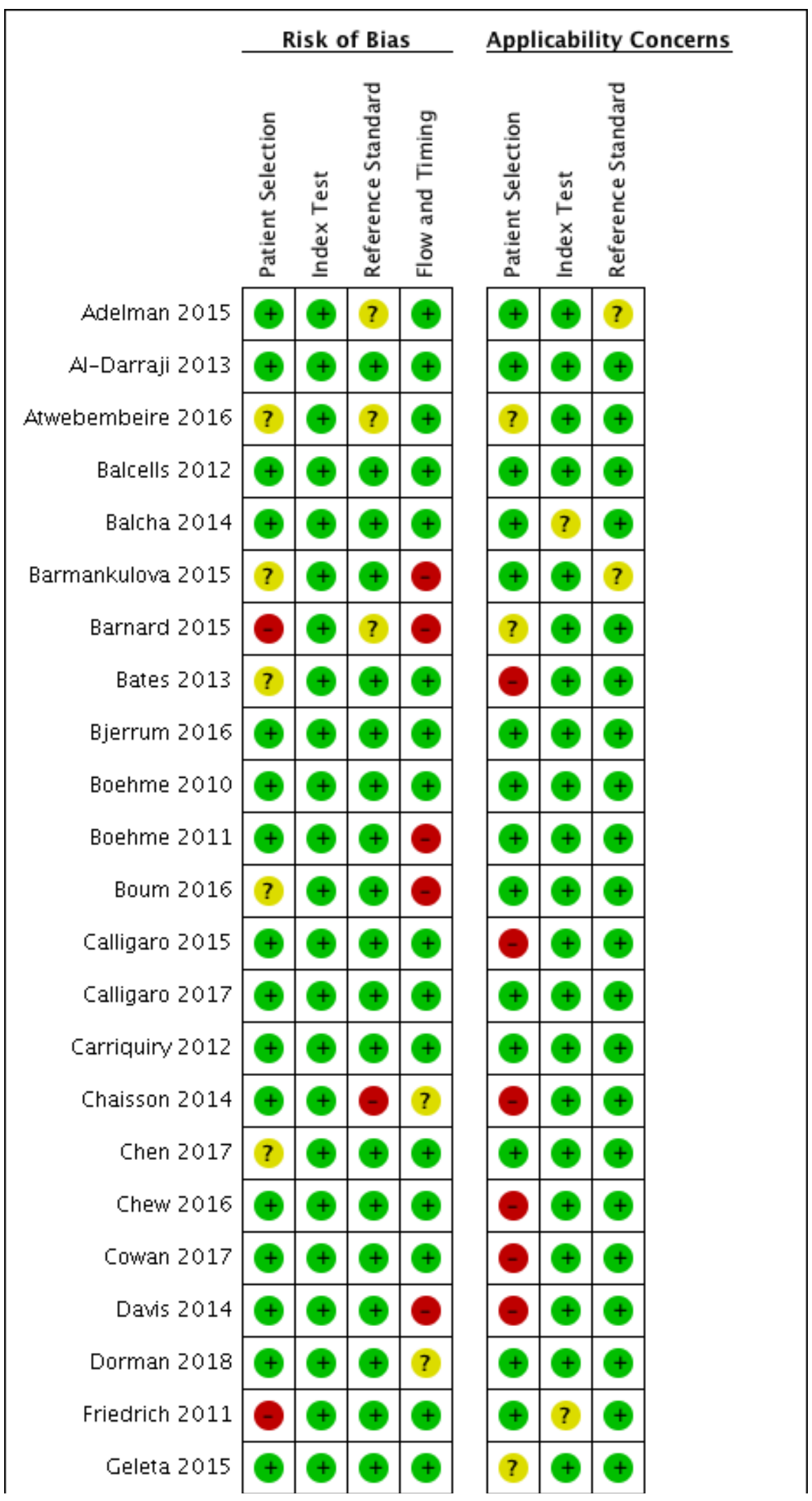

Xpert MTB/RIF and Xpert MTB/RIF Ultra for pulmonary tuberculosis and rifampicin resistance in adults (Review) 
Figure 5. (Continued)

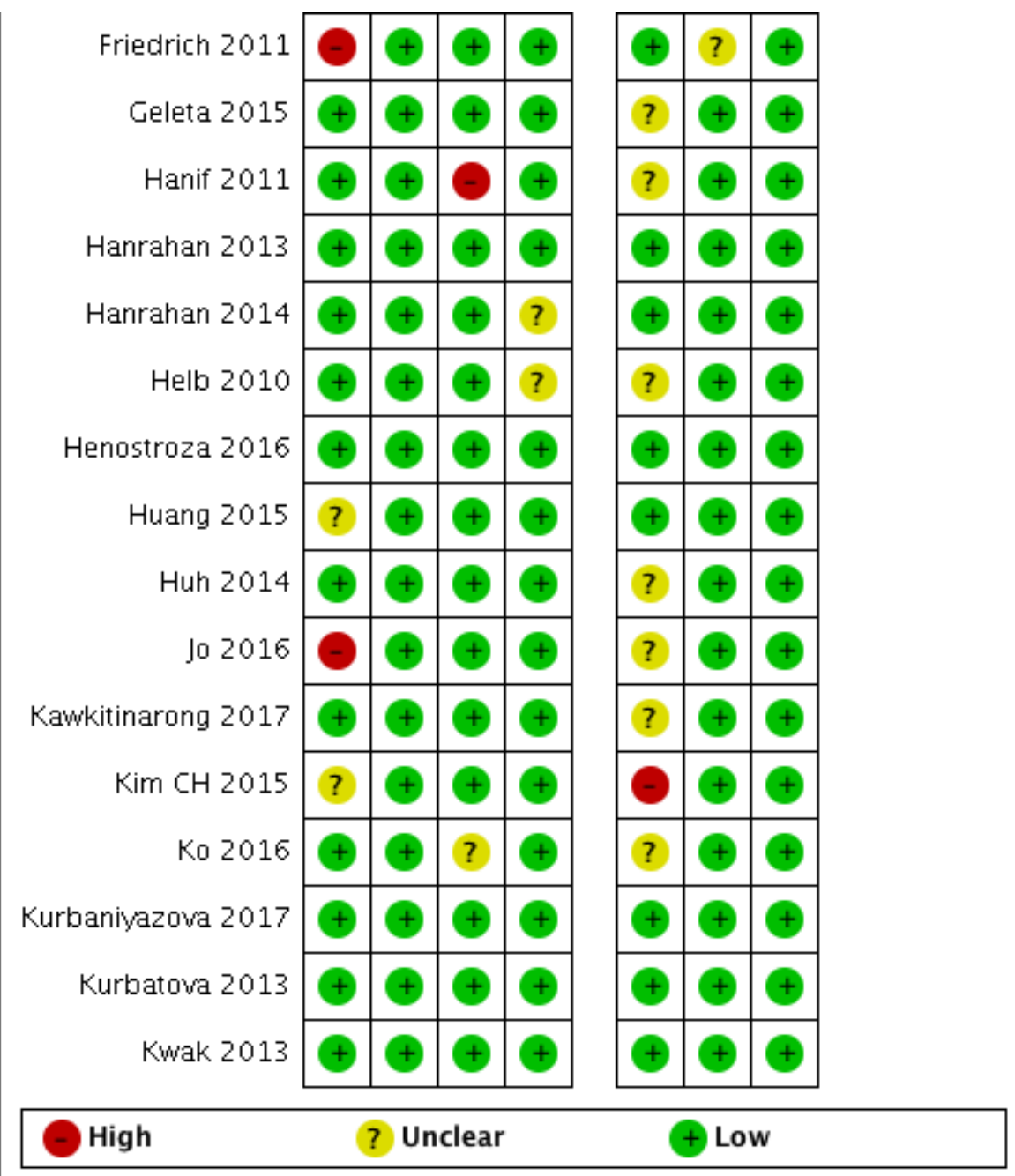


Figure 6. Risk of bias and applicability concerns summary for pulmonary tuberculosis detection: review authors' judgements about each domain for each included study, studies $L$ through $Z$.

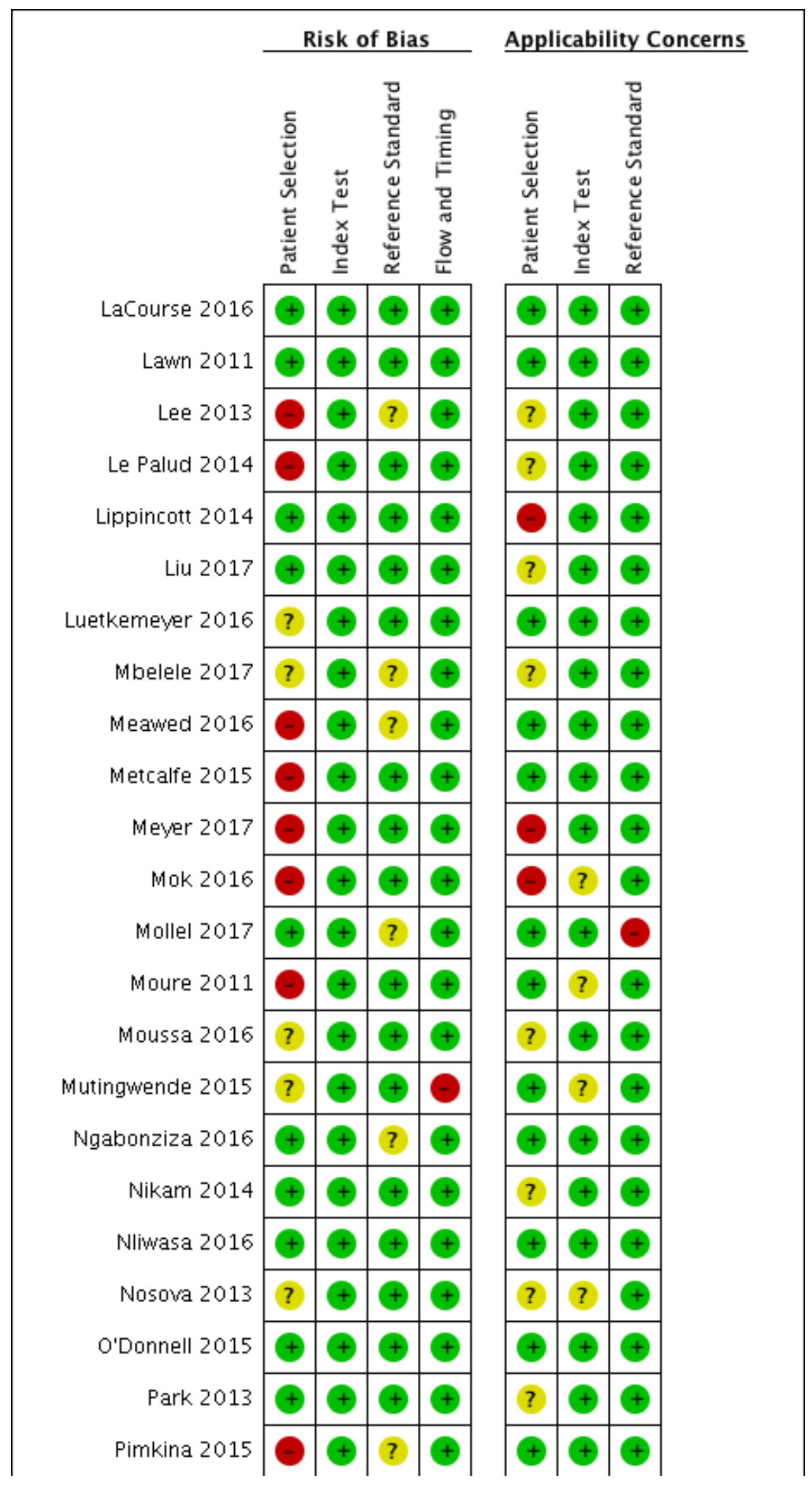

Xpert MTB/RIF and Xpert MTB/RIF Ultra for pulmonary tuberculosis and rifampicin resistance in adults (Review) 
Figure 6. (Continued)

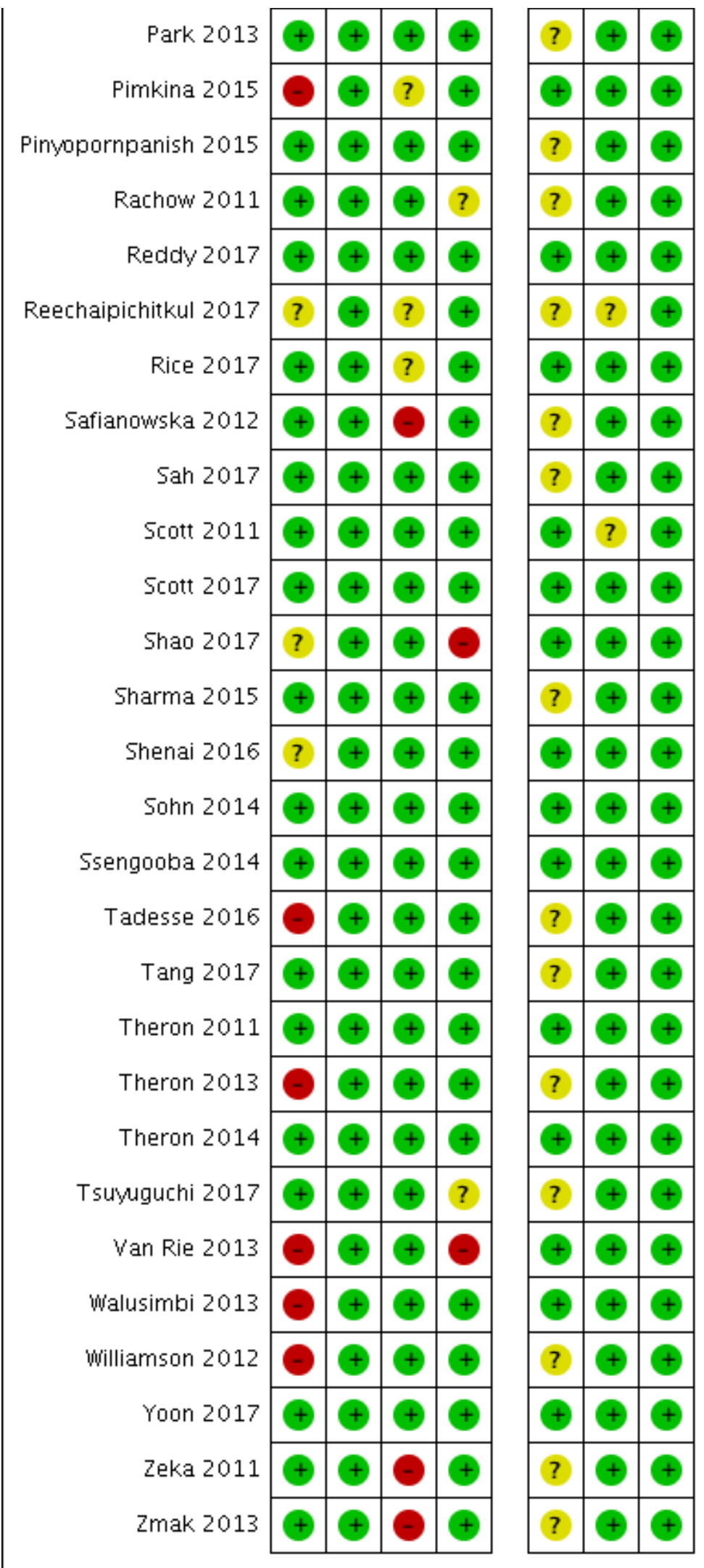

Xpert MTB/RIF and Xpert MTB/RIF Ultra for pulmonary tuberculosis and rifampicin resistance in adults (Review) 
Figure 6. (Continued)

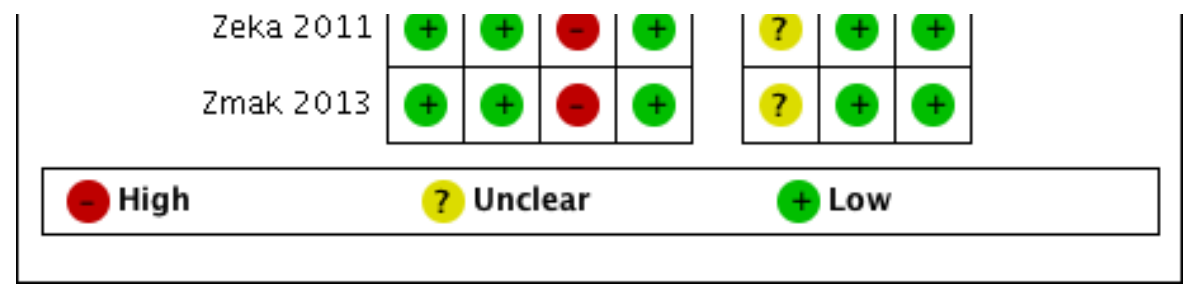


In the Patient Selection domain, we considered 55 studies (64\%) to have low risk of bias because the study enrolled a consecutive or random sample of eligible participants and avoided inappropriate exclusions. We considered 16 studies (19\%) to have high risk of bias because the study did not avoid inappropriate exclusions: 13 studies enrolled participants whose sputum specimens were primarily or exclusively smear-positive or smear-negative (Barnard 2015; Friedrich 2011; Jo 2016; Lee 2013; Le Palud 2014; Meyer 2017; Mok 2016; Moure 2011; Tadesse 2016; Theron 2013; Van Rie 2013; Walusimbi 2013a; Williamson 2012) and three studies exclusively enrolled participants who had previously received tuberculosis treatment (Meawed 2016; Metcalfe 2015; Pimkina 2015). In addition, we considered 15 studies (17\%) to have unclear risk of bias because the manner of participant selection was not stated (Atwebembeire 2016; Barmankulova 2015; Bates 2013a; Boum 2016; Chen 2017; Huang 2015; Kim CH 2015; Luetkemeyer 2016; Mbelele 2017; Moussa 2016; Mutingwende 2015; Nosova 2013a; Reechaipichitkul 2017; Shao 2017; Shenai 2016). With respect to applicability, we considered 46 studies (53\%) to have low concern because participants in these studies were evaluated in primary care facilities, local hospitals, or both settings (Adelman 2015; Al-Darraji 2013; Balcells 2012; Balcha 2014; Barmankulova 2015; Bjerrum 2016; Boehme 2010; Boehme 2011; Boum 2016; Calligaro 2017; Carriquiry 2012; Chen 2017; Dorman 2018; Friedrich 2011; Hanrahan 2013; Hanrahan 2014; Henostroza 2016; Huang 2015; Kurbaniyazova 2017; Kurbatova 2013; Kwak 2013; LaCourse 2016; Lawn 2011; Luetkemeyer 2016; Meawed 2016; Metcalfe 2015; Mollel 2017; Moure 2011; Mutingwende 2015; Ngabonziza 2016; Nliwasa 2016; O'Donnell 2015; Pimkina 2015; Reddy 2017; Rice 2017; Scott 2011; Scott 2017; Shao 2017; Shenai 2016; Sohn 2014; Ssengooba 2014; Theron 2011; Theron 2014a; Van Rie 2013; Walusimbi 2013a; Yoon 2017). We considered 10 studies (12\%) to have high concern because participants were evaluated exclusively as inpatients in tertiary care centres (Bates 2013a; Calligaro 2015; Chaisson 2014; Chew 2016; Cowan 2017; Davis 2014; Kim CH 2015; Lippincott 2014; Meyer 2017; Mok 2016). We considered 30 studies (35\%) to have unclear concern because we could not tell.

In the Index Test domain, we considered all studies to have low risk of bias. With respect to applicability, we considered most stud- ies to have low concern and eight studies to have unclear concern because the ratio of sample reagent to specimen volume differed from that recommended by the manufacturer or we could not tell (Balcells 2012; Friedrich 2011; Mok 2016; Moure 2011; Mutingwende 2015; Nosova 2013a; Reechaipichitkul 2017; Scott 2011).

In the Reference Standard domain, we considered 69 studies (80\%) to have low risk of bias because the results of the reference standard were interpreted without knowledge of the results of the index test. We considered five studies (6\%) to have high risk of bias because the results of the reference standard were not blinded (Chaisson 2014; Hanif 2011; Safianowska 2012; Zeka 2011; Zmak 2013) and the remaining 12 studies (14\%) to have unclear risk of bias because information about blinding was not reported. With respect to applicability (Reference Standard domain), we considered most studies to have low concern; we considered one study to have high concern because this study did not speciate mycobacteria isolated in culture (Mollel 2017) and two studies (2\%) to have unclear concern because we could not tell (Adelman 2015; Barmankulova 2015).

In the Flow and Timing domain, we considered 72 studies (84\%) to have low risk of bias because all participants were included in the analysis. We considered eight studies (9\%) to have high risk of bias: in seven studies, results for index or reference tests were not available for many participants (Barmankulova 2015; Barnard 2015; Boum 2016; Davis 2014; Mutingwende 2015; Shao 2017; Van Rie 2013); in one study, participants who were treated for tuberculosis on the basis of clinical and radiological findings (smear-negative, culture-negative) were not included in the analysis (Boehme 2011). We considered six studies (7\%) to have unclear risk of bias because we could not tell if all participants were included in the analysis (Chaisson 2014; Dorman 2018; Hanrahan 2014; Helb 2010; Rachow 2011; Tsuyuguchi 2017).

\section{Studies evaluating Xpert MTB/RIF and Xpert Ultra for detection of rifampicin resistance}

Figure 7 and Figure 8 show risk of bias and applicability concerns for 57 studies evaluating Xpert MTB/RIF and Xpert Ultra for rifampicin resistance detection.

\section{Figure 7. Risk of bias and applicability concerns graph for rifampicin resistance detection: review authors' judgements about each domain presented as percentages across included studies.}

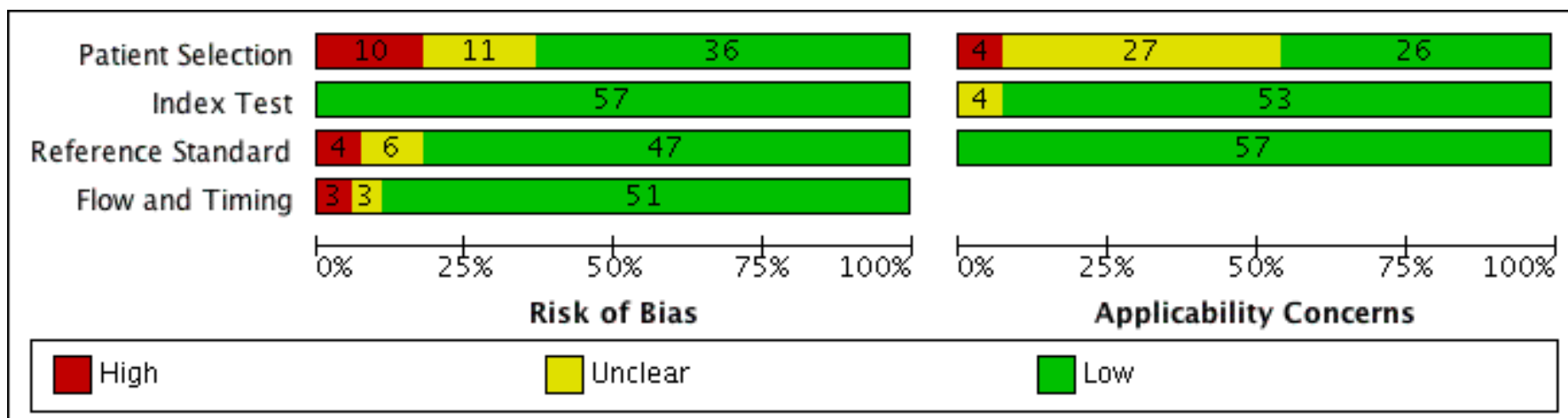


Figure 8. Risk of bias and applicability concerns summary for rifampicin resistance detection: review authors' judgements about each domain for each included study.

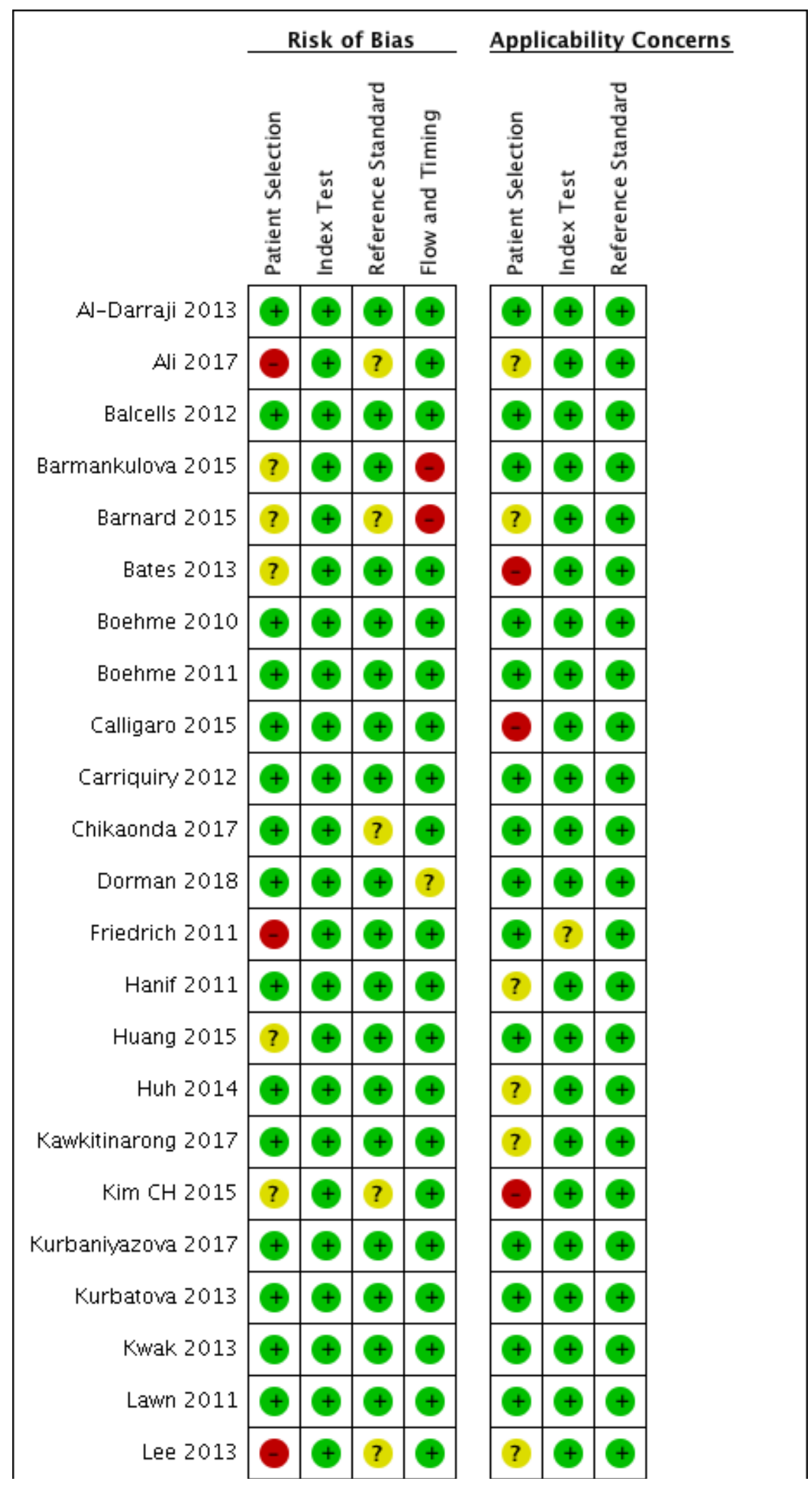

Xpert MTB/RIF and Xpert MTB/RIF Ultra for pulmonary tuberculosis and rifampicin resistance in adults (Review) 
Figure 8. (Continued)

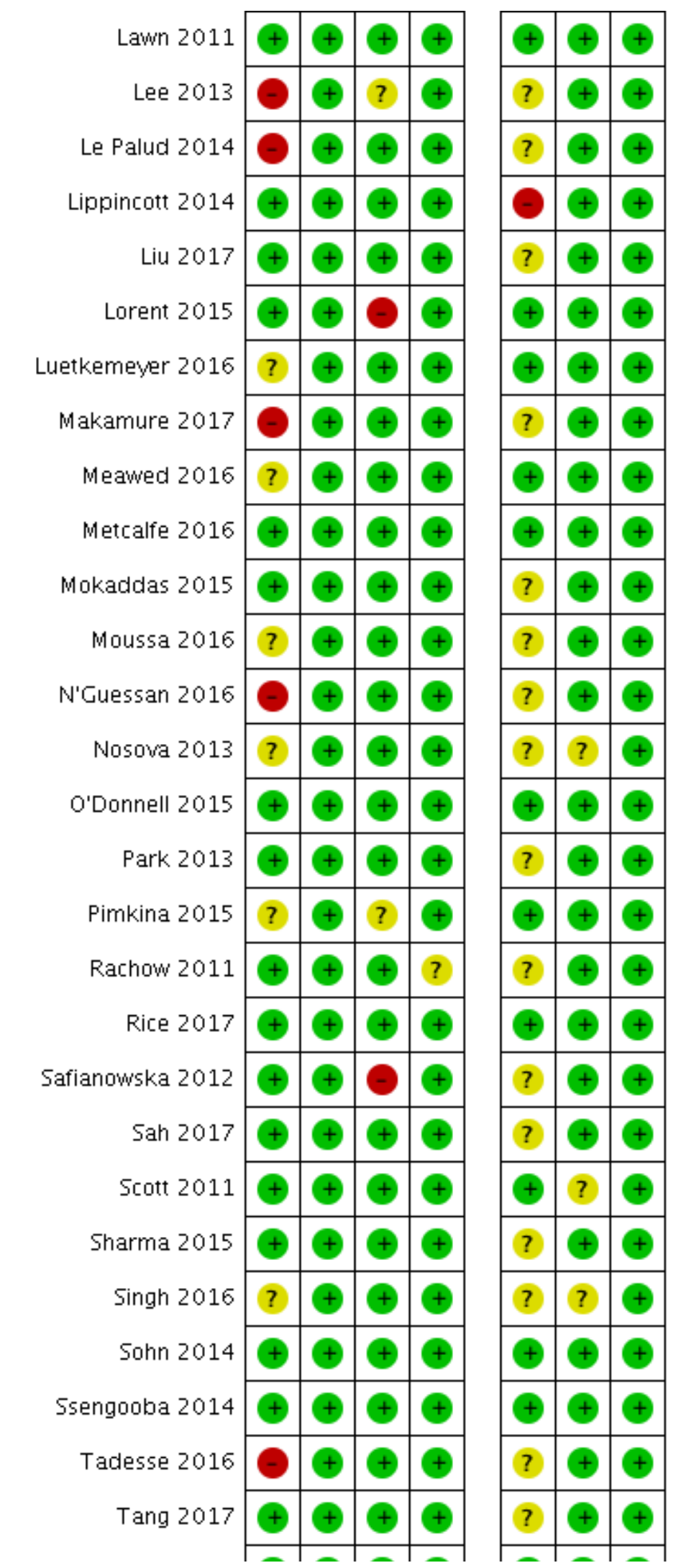


Figure 8. (Continued)

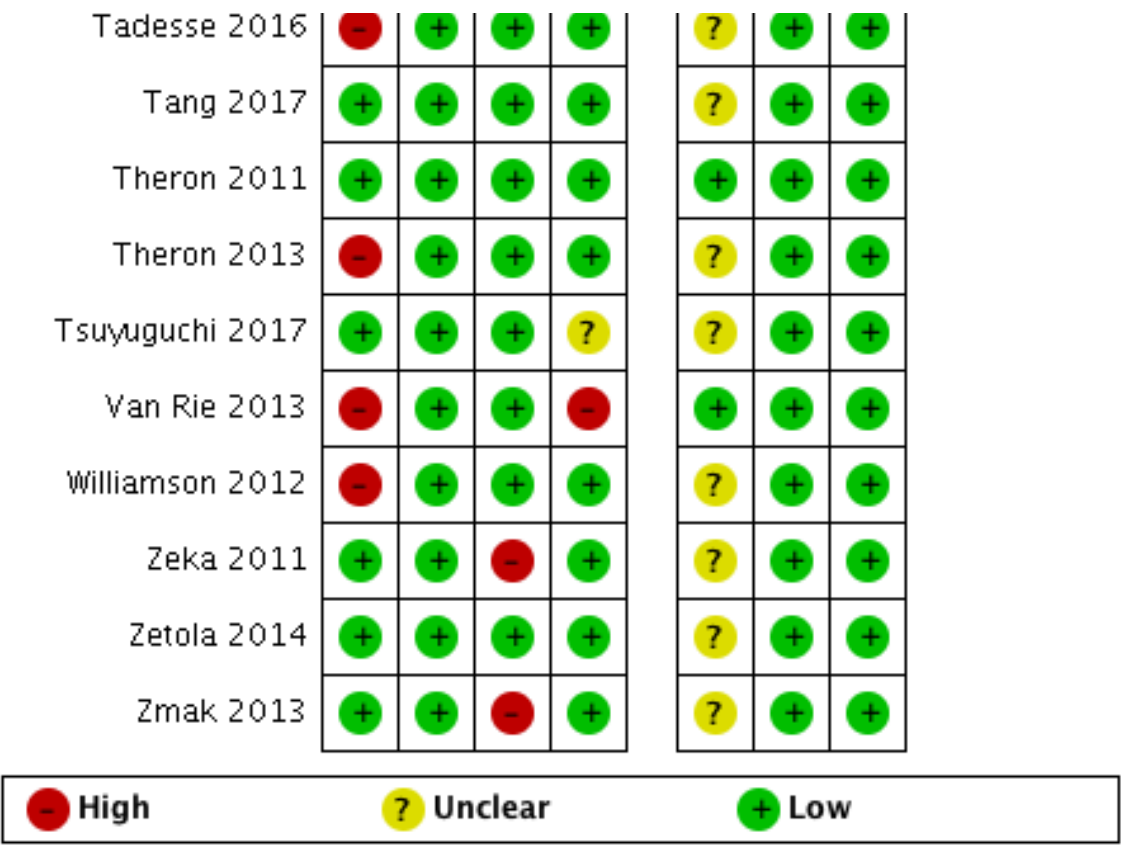


In the Patient Selection domain, we considered 36 studies (63\%) to have low risk of bias because the study enrolled a consecutive or random sample of eligible participants and avoided inappropriate exclusions. We considered 10 studies $(18 \%)$ to have high risk of bias because the study did not avoid inappropriate exclusions and instead enrolled participants preselected on the basis of their sputum specimens being either smear-positive or smear-negative or the study exclusively enrolled retreatment participants (Ali 2017; Friedrich 2011; Lee 2013; Le Palud 2014; Makamure 2017; N'Guessan 2016; Tadesse 2016; Theron 2013; Van Rie 2013; Williamson 2012). We considered 11 studies (19\%) to have unclear risk of bias because the manner of participant selection was not reported (Barmankulova 2015; Barnard 2015; Bates 2013a; Huang 2015; Kim CH 2015; Luetkemeyer 2016; Meawed 2016; Moussa 2016; Nosova 2013a; Pimkina 2015; Singh 2016). With respect to applicability, we considered 26 studies (46\%) to have low concern because participants in these studies were evaluated in primary care facilities, local hospitals, or both settings (Al-Darraji 2013; Balcells 2012; Barmankulova 2015; Boehme 2010; Boehme 2011; Carriquiry 2012; Chikaonda 2017; Dorman 2018; Friedrich 2011; Huang 2015; Kurbaniyazova 2017; Kurbatova 2013; Kwak 2013; Lawn 2011; Lorent 2015; Luetkemeyer 2016; Meawed 2016; Metcalfe 2016; O'Donnell 2015; Pimkina 2015; Rice 2017; Scott 2011; Sohn 2014; Ssengooba 2014; Theron 2011; Van Rie 2013). We considered four studies to have high concern (7\%) because participants were evaluated exclusively as inpatients in tertiary care centres (Bates 2013a; Calligaro 2015; Kim CH 2015; Lippincott 2014). We considered the remaining 27 studies (47\%) to have unclear concern because we could not tell.

In the Index Test domain, we considered all studies to have low risk of bias. With respect to applicability, we considered 53 studies $(93 \%)$ to have low concern and four studies $(7 \%)$ to have unclear concern because the ratio of sample reagent to specimen volume differed from that recommended by the manufacturer (Friedrich 2011; Nosova 2013a; Scott 2011; Singh 2016).

In the Reference Standard domain, we considered 47 studies (82\%) to have low risk of bias because the results of the reference standard were interpreted without knowledge of the results of the index test. We considered four studies (7\%) to have high risk of bias because the result of the reference standard was not blinded (Lorent 2015; Safianowska 2012; Zeka 2011; Zmak 2013) and the remaining six studies (11\%) to have unclear risk of bias because information was not reported. With respect to applicability in the Reference Standard domain, we considered all studies to have low concern because in these studies all specimens had already been speciated and identified as Mycobacterium tuberculosis.

In the Flow and Timing domain, we considered 51 studies (90\%) to have low risk of bias because all participants were included in the analysis. We considered three studies (5\%) to have high risk of bias because index and reference test results were not available for many participants (Barmankulova 2015; Barnard 2015; Van Rie 2013). We considered three studies (5\%) to have unclear risk of bias because we could not tell if all participants were included in the analysis (Dorman 2018; Rachow 2011; Tsuyuguchi 2017).

\section{Findings}

\section{Detection of PTB}

A total of 86 studies involving 42,091 participants evaluated the accuracy of Xpert MTB/RIF for PTB (Figure 9). For two multicentre studies (Boehme 2010; Boehme 2011) we provide two-by-two data for the individual centres in Appendix 2. The median number of participants in the studies was 256 (Interquartile range (IQR) 145 to 494). Key characteristics for the included studies are presented in Characteristics of included studies. 
Figure 9. Forest plots of Xpert sensitivity and specificity for detection of pulmonary tuberculosis. The individual studies are ordered by decreasing sensitivity. The squares represent the sensitivity and specificity of one study, the black line its confidence interval. FN: false-negative; FP: false-positive; TN: true-negative; TP: true-positive.

\begin{tabular}{|c|c|c|c|c|c|c|c|c|}
\hline Study & TP & FP & FN & TN & Sensitivity (95\% Cl) & Specificity (95\% Cl) & Sensitivity (95\% Cl) & Specificity (95\% Cl) \\
\hline Williamson 2012 & 67 & 0 & 0 & 22 & $1.00[0.95,1.00]$ & $1.00[0.85,1.00]$ & + & $\longrightarrow$ \\
\hline Mollel 2017 & 9 & 0 & 0 & 60 & $1.00[0.66,1.00]$ & $1.00[0.94,1.00]$ & & $\rightarrow$ \\
\hline Calligaro 2015 & 11 & 5 & 0 & 75 & $1.00[0.72,1.00]$ & $0.94[0.86,0.98]$ & & $\rightarrow$ \\
\hline Meawed 2016 & 53 & 1 & 1 & 3 & $0.98[0.90,1.00]$ & $0.75[0.19,0.99]$ & $\rightarrow$ & \\
\hline Carriquiry 2012 & 44 & 2 & 1 & 84 & $0.98[0.88,1.00]$ & $0.98[0.92,1.00]$ & $\rightarrow$ & $\rightarrow$ \\
\hline Sharma 2015 & 430 & 6 & 19 & 984 & $0.96[0.93,0.97]$ & $0.99[0.99,1.00]$ & घ & a \\
\hline Moussa 2016 & 67 & 3 & 3 & 145 & $0.96[0.88,0.99]$ & $0.98[0.94,1.00]$ & $\rightarrow$ & च \\
\hline Pinyopornpanish 2015 & 41 & 9 & 2 & 57 & $0.95[0.84,0.99]$ & $0.86[0.76,0.94]$ & $\rightarrow$ & $\rightarrow$ \\
\hline Kurbatova 2013 & 102 & 17 & 5 & 104 & $0.95[0.89,0.98]$ & $0.86[0.78,0.92]$ & $\rightarrow$ & $\rightarrow$ \\
\hline Shao 2017 & 106 & 31 & 6 & 151 & $0.95[0.89,0.98]$ & $0.83[0.77,0.88]$ & $\Rightarrow$ & $\Rightarrow$ \\
\hline O'Donnell 2015 & 112 & 1 & 7 & 35 & $0.94[0.88,0.98]$ & $0.97[0.85,1.00]$ & $\rightarrow$ & $\rightarrow$ \\
\hline Pimkina 2015 & 358 & 34 & 24 & 376 & $0.94[0.91,0.96]$ & $0.92[0.89,0.94]$ & - & 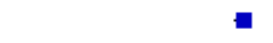 \\
\hline Lippincott 2014 & 13 & 1 & 1 & 484 & $0.93[0.66,1.00]$ & $1.00[0.99,1.00]$ & - & . \\
\hline Friedrich 2011 & 117 & 0 & 9 & 0 & $0.93[0.87,0.97]$ & Not estimable & $\Rightarrow$ & \\
\hline Kurbaniyazova 2017 & 1577 & 99 & 124 & 934 & $0.93[0.91,0.94]$ & $0.90[0.88,0.92]$ & - & - \\
\hline Liu 2017 & 405 & 231 & 32 & 2428 & $0.93[0.90,0.95]$ & $0.91[0.90,0.92]$ & - & घ \\
\hline Theron 2013 & 25 & 5 & 2 & 120 & $0.93[0.76,0.99]$ & $0.96[0.91,0.99]$ & $\rightarrow$ & $\rightarrow$ \\
\hline Ko 2016 & 97 & 12 & 8 & 132 & $0.92[0.86,0.97]$ & $0.92[0.86,0.96]$ & $\rightarrow$ & $\rightarrow$ \\
\hline Barnard 2015 & 36 & 9 & 3 & 64 & $0.92[0.79,0.98]$ & $0.88[0.78,0.94]$ & $\rightarrow$ & $=$ \\
\hline Davis 2014 & 12 & 3 & 1 & 140 & $0.92[0.64,1.00]$ & $0.98[0.94,1.00]$ & & 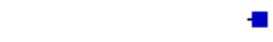 \\
\hline Boehme 2010 & 675 & 26 & 57 & 681 & $0.92[0.90,0.94]$ & $0.96[0.95,0.98]$ & घ & a \\
\hline Jo 2016 & 59 & 47 & 5 & 209 & $0.92[0.83,0.97]$ & $0.82[0.76,0.86]$ & $\rightarrow$ & $\rightarrow$ \\
\hline Nosova $2013 a$ & 47 & 0 & 4 & 86 & $0.92[0.81,0.98]$ & $1.00[0.96,1.00]$ & $\rightarrow$ & $\rightarrow$ \\
\hline Metcalfe 2015 & 82 & 8 & 7 & 52 & $0.92[0.84,0.97]$ & $0.87[0.75,0.94]$ & $\rightarrow$ & \\
\hline Boum 2016 & 194 & 22 & 17 & 654 & $0.92[0.87,0.95]$ & $0.97[0.95,0.98]$ & $=$ & घ \\
\hline Scott 2017 & 57 & 3 & 5 & 128 & $0.92[0.82,0.97]$ & $0.98[0.93,1.00]$ & $\rightarrow$ & 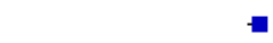 \\
\hline Shenai 2016 & 89 & 5 & 8 & 234 & $0.92[0.84,0.96]$ & $0.98[0.95,0.99]$ & $\rightarrow$ & घ \\
\hline Balcells 2012 & 11 & 1 & 1 & 147 & $0.92[0.62,1.00]$ & $0.99[0.96,1.00]$ & & - \\
\hline Huh 2014 & 95 & 10 & 9 & 157 & $0.91[0.84,0.96]$ & $0.94[0.89,0.97]$ & 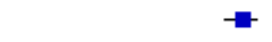 & - \\
\hline Kawkitinarong 2017 & 227 & 6 & 23 & 133 & $0.91[0.87,0.94]$ & $0.96[0.91,0.98]$ & - & $\rightarrow$ \\
\hline Boehme 2011 & 933 & 30 & 100 & 2846 & $0.90[0.88,0.92]$ & $0.99[0.99,0.99]$ & घ & घ \\
\hline Hanif 2011 & 54 & 0 & 6 & 146 & $0.90[0.79,0.96]$ & $1.00[0.98,1.00]$ & $\rightarrow$ & 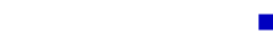 \\
\hline Rice 2017 & 120 & 2 & 14 & 600 & $0.90[0.83,0.94]$ & $1.00[0.99,1.00]$ & $\rightarrow$ & घ \\
\hline Nikam 2014 & 135 & 59 & 16 & 64 & $0.89[0.83,0.94]$ & $0.52[0.43,0.61]$ & $\rightarrow$ & $\rightarrow-$ \\
\hline Chaisson 2014 & 8 & 0 & 1 & 133 & $0.89[0.52,1.00]$ & $1.00[0.97,1.00]$ & & $=$ \\
\hline Zeka 2011 & 31 & 0 & 4 & 68 & $0.89[0.73,0.97]$ & $1.00[0.95,1.00]$ & & - \\
\hline $\mathrm{Kim} \mathrm{CH} 2015$ & 46 & 5 & 6 & 348 & $0.88[0.77,0.96]$ & $0.99[0.97,1.00]$ & $\rightarrow$ & घ \\
\hline Rachow 2011 & 61 & 8 & 8 & 172 & $0.88[0.78,0.95]$ & $0.96[0.91,0.98]$ & $\rightarrow$ & $=$ \\
\hline Huang 2015 & 166 & 31 & 22 & 159 & $0.88[0.83,0.93]$ & $0.84[0.78,0.89]$ & $\Rightarrow$ & $\rightarrow$ \\
\hline Safianowska 2012 & 15 & 1 & 2 & 127 & $0.88[0.64,0.99]$ & $0.99[0.96,1.00]$ & $\longrightarrow$ & - \\
\hline Tsuyuguchi 2017 & 197 & 6 & 30 & 180 & $0.87[0.82,0.91]$ & $0.97[0.93,0.99]$ & $\Rightarrow$ & 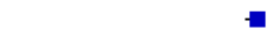 \\
\hline Scott 2011 & 58 & 3 & 9 & 107 & $0.87[0.76,0.94]$ & $0.97[0.92,0.99]$ & $\rightarrow$ & 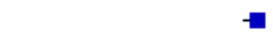 \\
\hline Mbelele 2017 & 73 & 25 & 12 & 152 & $0.86[0.77,0.92]$ & $0.86[0.80,0.91]$ & $\rightarrow$ & $\rightarrow$ \\
\hline Zmak 2013 & 6 & 0 & 1 & 110 & $0.86[0.42,1.00]$ & $1.00[0.97,1.00]$ & $\longrightarrow$ & $=$ \\
\hline Mutingwende 2015 & 191 & 12 & 33 & 60 & $0.85[0.80,0.90]$ & $0.83[0.73,0.91]$ & & $\rightarrow-$ \\
\hline Cowan 2017 & 17 & 0 & 3 & 298 & $0.85[0.62,0.97]$ & $1.00[0.99,1.00]$ & $\longrightarrow-$ & E \\
\hline Chew 2016 & 34 & 1 & 6 & 197 & $0.85[0.70,0.94]$ & $0.99[0.97,1.00]$ & $\longrightarrow$ & ш \\
\hline Reechaipichitkul 2017 & 53 & 5 & 10 & 57 & $0.84[0.73,0.92]$ & $0.92[0.82,0.97]$ & $\rightarrow$ & $\rightarrow$ \\
\hline Tang 2017 & 68 & 15 & 13 & 129 & $0.84[0.74,0.91]$ & $0.90[0.83,0.94]$ & $\rightarrow$ & $\Rightarrow$ \\
\hline Calligaro 2017 & 35 & 13 & 7 & 348 & $0.83[0.69,0.93]$ & $0.96[0.94,0.98]$ & $\longrightarrow$ & - \\
\hline Chen 2017 & 5 & 3 & 1 & 724 & $0.83[0.36,1.00]$ & $1.00[0.99,1.00]$ & $\longrightarrow$ & घ \\
\hline Theron $2014 a$ & 154 & 27 & 31 & 517 & $0.83[0.77,0.88]$ & $0.95[0.93,0.97]$ & $\rightarrow$ & - \\
\hline Dorman 2018 & 383 & 17 & 79 & 960 & $0.83[0.79,0.86]$ & $0.98[0.97,0.99]$ & 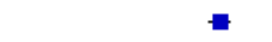 & घ \\
\hline Park 2013 & 19 & 6 & 4 & 291 & $0.83[0.61,0.95]$ & $0.98[0.96,0.99]$ & $\longrightarrow-$ & घ \\
\hline Sah 2017 & 32 & 5 & 7 & 61 & $0.82[0.66,0.92]$ & $0.92[0.83,0.97]$ & $\longrightarrow$ & $\rightarrow$ \\
\hline Helb 2010 & 67 & 0 & 15 & 25 & $0.82[0.72,0.89]$ & $1.00[0.86,1.00]$ & $\rightarrow$ & $\rightarrow$ \\
\hline Lee 2013 & 31 & 0 & 7 & 94 & $0.82[0.66,0.92]$ & $1.00[0.96,1.00]$ & $\longrightarrow-$ & $\Rightarrow$ \\
\hline Luetkemeyer 2016 & 175 & 10 & 40 & 735 & $0.81[0.76,0.86]$ & $0.99[0.98,0.99]$ & $\rightarrow$ & - \\
\hline Barmankulnva 2015 & 191 & 1 & 44 & 55 & 081 in 76 ก 861 & 098 in 901000 & $\Rightarrow$ & $\rightarrow$ \\
\hline
\end{tabular}

Xpert MTB/RIF and Xpert MTB/RIF Ultra for pulmonary tuberculosis and rifampicin resistance in adults (Review)

Copyright $\odot 2019$ The Authors. Cochrane Database of Systematic Reviews published by John Wiley \& Sons, Ltd. on behalf of The Cochrane Collaboration.

Kwak 2013

Theron 2011

Moure 2011

Niwasa 2016

Bjerrum 2016 $\begin{array}{lllll}124 & 20 & 32 & 505 & 0.79[0.72,0.86]\end{array}$

$\begin{array}{lllll}111 & 19 & 30 & 320 & 0.79[0.71,0.85]\end{array}$

$\begin{array}{lllll}61 & 0 & 17 & 29 & 0.78[0.67,0.87]\end{array}$

$\begin{array}{lllll}31 & 9 & 9 & 181 & 0.78[0.62,0.89]\end{array}$

$\begin{array}{lllll}27 & 5 & 8 & 155 & 0.77[0.60,0.90]\end{array}$
$0.96[0.94,0.98]$ $0.94[0.91,0.97]$ $1.00[0.88,1.00]$ $0.95[0.91,0.98]$ $0.97[0.93,0.99]$ 


\section{A. Primary analysis, Xpert MTB/RIF and Xpert Ultra for detection of PTB}

\section{A.1. Xpert MTB/RIF}

For the 86 studies, sensitivity estimates ranged from $43 \%$ to $100 \%$ (Figure 9). Differences in enrolment criteria (different populations targeted), disease severity, and settings were notable in several studies with low sensitivity: LaCourse 2016 (sensitivity 43\%) included HIV-positive pregnant women accessing prevention of mother-to-child transmission services (no tuberculosis symptoms reported) and sensitivity was based on a small number of tuberculosis cases (seven tuberculosis cases). Sohn 2014 (sensitivity 44\%) evaluated induced sputum specimens from participants with presumptive PTB, most of whom were asymptomatic. Atwebembeire 2016 (sensitivity 48\%) only included adults unable to produce sputum and frozen specimens. Adelman 2015 and Al-Darraji 2013 included few tuberculosis cases. Yoon 2017 enrolled HIV-positive people initiating antiretroviral therapy. Lawn 2011 included HIVpositive participants irrespective of tuberculosis symptoms. Specificity varied less than sensitivity, with specificity estimates ranging from $52 \%$ to $100 \%$, although most specificity estimates were greater than 90\% (Figure 9). Nikam 2014 (specificity 52\%) was an outlier, and although we corresponded with the study author we could not explain the low specificity in this study.

\section{A.1.a. Xpert MTB/RIF accuracy, all studies meeting inclusion criteria}

In this meta-analysis, we included 85 studies involving 41,965 participants. We excluded one study that only reported sensitivity data (Friedrich 2011). Xpert pooled sensitivity and specificity (95\% credible interval (Crl)) were $85 \%$ (82\% to $87 \%)$ and $98 \%(97 \%$ to $98 \%)$, respectively (Table 1 ).

\section{A.1.b. Xpert MTB/RIF accuracy, limited to studies with unselected participants}

We included 70 studies involving 37,237 unselected participants (Adelman 2015; Al-Darraji 2013; Atwebembeire 2016; Balcells 2012; Balcha 2014; Barmankulova 2015; Bates 2013a; Bjerrum 2016; Boehme 2010; Boehme 2011; Boum 2016; Calligaro 2015; Calligaro 2017; Carriquiry 2012; Chaisson 2014; Chen 2017; Chew 2016; Cowan 2017; Davis 2014; Dorman 2018; Geleta 2015; Hanif 2011; Hanrahan 2013; Hanrahan 2014; Helb 2010; Henostroza 2016; Huang 2015; Huh 2014; Kawkitinarong 2017; Kim CH 2015; Ko 2016; Kurbaniyazova 2017; Kurbatova 2013; Kwak 2013; LaCourse 2016; Lawn 2011; Lippincott 2014; Liu 2017; Luetkemeyer 2016; Mbelele 2017; Mollel 2017; Moussa 2016; Mutingwende 2015; Ngabonziza 2016; Nikam 2014; Nliwasa 2016; Nosova 2013a; O'Donnell 2015; Park 2013; Pinyopornpanish 2015; Rachow 2011; Reddy 2017; Reechaipichitkul 2017; Rice 2017; Safianowska 2012; Sah 2017; Scott 2011; Scott 2017; Shao 2017; Sharma 2015; Shenai 2016; Sohn 2014; Ssengooba 2014; Tang 2017; Theron 2011; Theron 2014a; Tsuyuguchi 2017; Yoon 2017; Zeka 2011; Zmak 2013). We excluded 16 studies, i.e. 13 studies that preselected participants on the basis of a prior smear microscopy result (participants whose sputum specimens were primarily or exclusively smear-positive or smear-negative) (Barnard 2015; Friedrich 2011; Jo 2016; Lee 2013; Le Palud 2014; Meyer 2017; Mok 2016; Moure 2011; Tadesse 2016; Theron 2013; Van Rie 2013; Walusimbi 2013a; Williamson 2012) and three studies that preselected participants who had previously received tuberculosis treatment (Meawed 2016; Metcalfe 2015; Pimkina 2015) (Figure 10). 
Figure 10. Forest plots of Xpert sensitivity and specificity for detection of pulmonary tuberculosis in studies with unselected participants. The individual studies are ordered by decreasing sensitivity. The squares represent the 
sensitivity and specificity of one study, the black line its confidence interval. FN: false-negative; FP: false-positive; TN: true-negative; TP: true-positive.

\begin{tabular}{|c|c|c|c|c|c|}
\hline Study & TP & FP & FN & TN & Sensitivity $(95 \% \mathrm{CI})$ \\
\hline Calligaro 2015 & 11 & 5 & 0 & 75 & $1.00[0.72,1.00]$ \\
\hline Mollel 2017 & 9 & 0 & 0 & 60 & $1.00[0.66,1.00]$ \\
\hline Carriquiry 2012 & 44 & 2 & 1 & 84 & $0.98[0.88,1.00]$ \\
\hline Sharma 2015 & 430 & 6 & 19 & 984 & $0.96[0.93,0.97]$ \\
\hline Moussa 2016 & 67 & 3 & 3 & 145 & $0.96[0.88,0.99]$ \\
\hline Pinyopornpanish 2015 & 41 & 9 & 2 & 57 & $0.95[0.84,0.99]$ \\
\hline Kurbatova 2013 & 102 & 17 & 5 & 104 & $0.95[0.89,0.98]$ \\
\hline Shao 2017 & 106 & 31 & 6 & 151 & $0.95[0.89,0.98]$ \\
\hline O'Donnell 2015 & 112 & 1 & 7 & 35 & $0.94[0.88,0.98]$ \\
\hline Lippincott 2014 & 13 & 1 & 1 & 484 & $0.93[0.66,1.00]$ \\
\hline Kurbaniyazova 2017 & 1577 & 99 & 124 & 934 & $0.93[0.91,0.94]$ \\
\hline Liu 2017 & 405 & 231 & 32 & 2428 & $0.93[0.90,0.95]$ \\
\hline Ko 2016 & 97 & 12 & 8 & 132 & $0.92[0.86,0.97]$ \\
\hline Davis 2014 & 12 & 3 & 1 & 140 & $0.92[0.64,1.00]$ \\
\hline Boehme 2010 & 675 & 26 & 57 & 681 & $0.92[0.90,0.94]$ \\
\hline Nosova 2013 & 47 & 0 & 4 & 86 & $0.92[0.81,0.98]$ \\
\hline Boum 2016 & 194 & 22 & 17 & 654 & $0.92[0.87,0.95]$ \\
\hline Scott 2017 & 57 & 3 & 5 & 128 & $0.92[0.82,0.97]$ \\
\hline Shenai 2016 & 89 & 5 & 8 & 234 & $0.92[0.84,0.96]$ \\
\hline Balcells 2012 & 11 & 1 & 1 & 147 & $0.92[0.62,1.00]$ \\
\hline Huh 2014 & 95 & 10 & 9 & 157 & $0.91[0.84,0.96]$ \\
\hline Kawkitinarong 2017 & 227 & 6 & 23 & 133 & $0.91[0.87,0.94]$ \\
\hline Boehme 2011 & 933 & 30 & 100 & 2846 & $0.90[0.88,0.92]$ \\
\hline Hanif 2011 & 54 & 0 & 6 & 146 & $0.90[0.79,0.96]$ \\
\hline Rice 2017 & 120 & 2 & 14 & 600 & $0.90[0.83,0.94]$ \\
\hline Nikam 2014 & 135 & 59 & 16 & 64 & $0.89[0.83,0.94]$ \\
\hline Chaisson 2014 & 8 & 0 & 1 & 133 & $0.89[0.52,1.00]$ \\
\hline Zeka 2011 & 31 & 0 & 4 & 68 & $0.89[0.73,0.97]$ \\
\hline $\mathrm{Kim}$ CH 2015 & 46 & 5 & 6 & 348 & $0.88[0.77,0.96]$ \\
\hline Rachow 2011 & 61 & 8 & 8 & 172 & $0.88[0.78,0.95]$ \\
\hline Huang 2015 & 166 & 31 & 22 & 159 & $0.88[0.83,0.93]$ \\
\hline Safianowska 2012 & 15 & 1 & 2 & 127 & $0.88[0.64,0.99]$ \\
\hline Tsuyuguchi 2017 & 197 & 6 & 30 & 180 & $0.87[0.82,0.91]$ \\
\hline Scott 2011 & 58 & 3 & 9 & 107 & $0.87[0.76,0.94]$ \\
\hline Mbelele 2017 & 73 & 25 & 12 & 152 & $0.86[0.77,0.92]$ \\
\hline Zmak 2013 & 6 & 0 & 1 & 110 & $0.86[0.42,1.00]$ \\
\hline Mutingwende 2015 & 191 & 12 & 33 & 60 & $0.85[0.80,0.90]$ \\
\hline Chew 2016 & 34 & 1 & 6 & 197 & $0.85[0.70,0.94]$ \\
\hline Cowan 2017 & 17 & 0 & 3 & 298 & $0.85[0.62,0.97]$ \\
\hline Reechaipichitkul 2017 & 53 & 5 & 10 & 57 & $0.84[0.73,0.92]$ \\
\hline Tang 2017 & 68 & 15 & 13 & 129 & $0.84[0.74,0.91]$ \\
\hline Chen 2017 & 5 & 3 & 1 & 724 & $0.83[0.36,1.00]$ \\
\hline Calligaro 2017 & 35 & 13 & 7 & 348 & $0.83[0.69,0.93]$ \\
\hline Theron 2014 & 154 & 27 & 31 & 517 & $0.83[0.77,0.88]$ \\
\hline Dorman 2018 & 383 & 17 & 79 & 960 & $0.83[0.79,0.86]$ \\
\hline Park 2013 & 19 & 6 & 4 & 291 & $0.83[0.61,0.95]$ \\
\hline Sah 2017 & 32 & 5 & 7 & 61 & $0.82[0.66,0.92]$ \\
\hline Helb 2010 & 67 & 0 & 15 & 25 & $0.82[0.72,0.89]$ \\
\hline Luetkemeyer 2016 & 175 & 10 & 40 & 735 & $0.81[0.76,0.86]$ \\
\hline Barmankulova 2015 & 191 & 1 & 44 & 55 & $0.81[0.76,0.86]$ \\
\hline Bates 2013 & 21 & 2 & 5 & 66 & $0.81[0.61,0.93]$ \\
\hline Ngabonziza 2016 & 77 & 5 & 19 & 499 & $0.80[0.71,0.88]$ \\
\hline Kwak 2013 & 124 & 20 & 32 & 505 & $0.79[0.72,0.86]$ \\
\hline Theron 2011 & 111 & 19 & 30 & 320 & $0.79[0.71,0.85]$ \\
\hline Nliwasa 2016 & 31 & 9 & 9 & 181 & $0.78[0.62,0.89]$ \\
\hline Bjerrum 2016 & 27 & 5 & 8 & 155 & $0.77[0.60,0.90]$ \\
\hline Ssengooba 2014 & 94 & 10 & 29 & 291 & $0.76[0.68,0.84]$ \\
\hline Reddy 2017 & 117 & 35 & 37 & 458 & $0.76[0.68,0.82]$ \\
\hline Hanrahan 2014 & 299 & 38 & 107 & 1638 & $0.74[0.69,0.78]$ \\
\hline Balcha 2014 & 81 & 13 & 41 & 677 & $0.66[0.57,0.75]$ \\
\hline Hanrahan 2013 & 42 & 2 & 22 & 487 & $0.66[0.53,0.77]$ \\
\hline Geleta 2015 & 38 & 6 & 20 & 156 & $0.66[0.52,0.78]$ \\
\hline Henostroza 2016 & 39 & 5 & 23 & 266 & $0.63[0.50,0.75]$ \\
\hline
\end{tabular}

\section{$0.94[0.86,0.98]$}

$1.00[0.94,1.00]$

$0.98[0.92,1.00]$

$0.99[0.99,1.00]$

$0.98[0.94,1.00]$

$0.86[0.76,0.94]$

$0.86[0.78,0.92]$

$0.83[0.77,0.88]$

$0.97[0.85,1.00]$

$1.00[0.99,1.00]$

$0.90[0.88,0.92]$

$0.91[0.90,0.92]$

$0.92[0.86,0.96]$

$0.98[0.94,1.00]$

$0.96[0.95,0.98]$

$1.00[0.96,1.00]$

$0.97[0.95,0.98]$

$0.98[0.93,1.00]$

$0.98[0.95,0.99]$

$0.99[0.96,1.00]$

$0.94[0.89,0.97]$

$0.96[0.91,0.98]$

$0.99[0.99,0.99]$

$1.00[0.98,1.00]$

$1.00[0.99,1.00]$

$0.52[0.43,0.61]$

$1.00[0.97,1.00]$

$1.00[0.95,1.00]$

$0.99[0.97,1.00]$

$0.96[0.91,0.98]$

$0.84[0.78,0.89]$

$0.99[0.96,1.00]$

$0.97[0.93,0.99]$

$0.97[0.92,0.99]$

$0.86[0.80,0.91]$

$1.00[0.97,1.00]$

$0.83[0.73,0.91]$

$0.99[0.97,1.00]$

$1.00[0.99,1.00]$

$0.92[0.82,0.97]$

$0.90[0.83,0.94]$

$1.00[0.99,1.00]$

$0.96[0.94,0.98]$

$0.95[0.93,0.97]$

$0.98[0.97,0.99]$

$0.98[0.96,0.99]$

$0.92[0.83,0.97]$

$1.00[0.86,1.00]$

$0.99[0.98,0.99]$

$0.98[0.90,1.00]$

$0.97[0.90,1.00]$

$0.99[0.98,1.00]$

$0.96[0.94,0.98]$

$0.94[0.91,0.97]$

$0.95[0.91,0.98]$

$0.97[0.93,0.99]$

$0.97[0.94,0.98]$

$0.93[0.90,0.95]$

$0.98[0.97,0.98]$

$0.98[0.97,0.99]$

$1.00[0.99,1.00]$

$0.96[0.92,0.99]$

$0.98[0.96,0.99]$
Sensitivity $(95 \%$ CI) Specificity $(95 \% \mathrm{CI})$

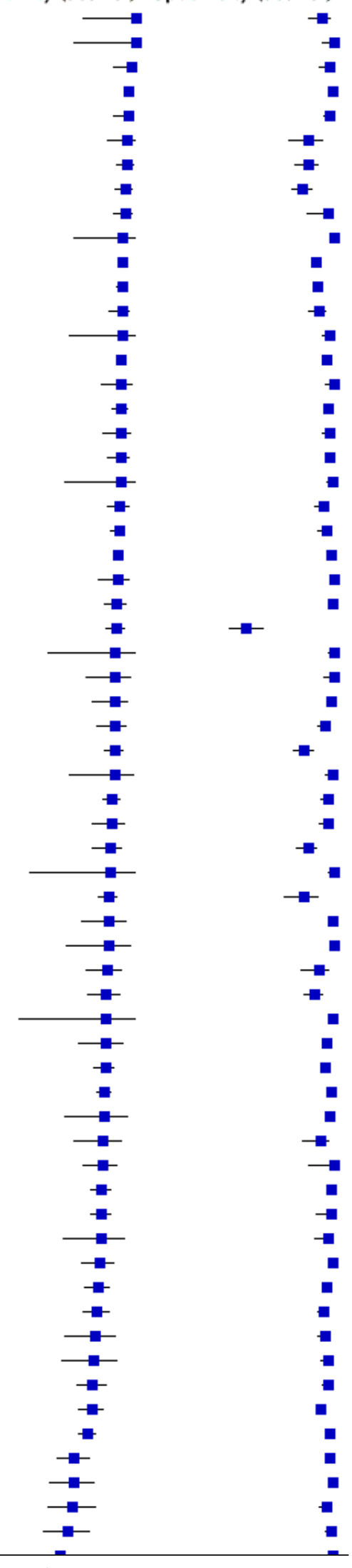

32

Xpert MTB/RIF and Xpert MTB/RIF Ultra for pulmonary tuberculosis and rifampicin resistance in adults (Review)

Copyright (C 2019 The Authors. Cochrane Database of Systematic Reviews published by John Wiley \& Sons, Ltd. on behalf of The Cochrane Collaboration.

$\begin{array}{lrrrrrr}\text { Atwebembeire 2016 } & 16 & 1 & 17 & 70 & 0.48[0.31,0.66] & 0.99[0.92,1.00] \\ \text { Sohn 2014 } & 11 & 1 & 14 & 475 & 0.44[0.24,0.65] & 1.00[0.99,1.00] \\ \text { LaCourse 2016 } & 3 & 1 & 4 & 280 & 0.43[0.10,0.82] & 1.00[0.98,1.00]\end{array}$

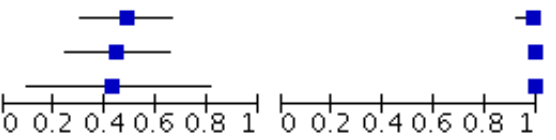


Xpert MTB/RIF pooled sensitivity and specificity ( $95 \% \mathrm{Crl}$ ) were $85 \%$ ( $82 \%$ to $88 \%$ ) and $98 \%$ (97\% to $98 \%$ ), essentially the same as the estimates obtained when including all studies regardless of their selection criteria (Table 1).

Figure 11 presents the pooled and predicted sensitivity and specificity estimates together with the credible and prediction regions for Xpert MTB/RIF for PTB. The summary point (pooled value) ap- pears close to the upper left-hand corner of the plot, suggesting high accuracy of Xpert MTB/RIF for detection of PTB. The $95 \%$ credible region around the summary point of sensitivity and specificity, the region that contains likely combinations of the pooled sensitivity and specificity, is relatively narrow. The $95 \%$ prediction region is wider, displaying more uncertainty as to where the likely values of sensitivity and specificity might occur in a future study. 
Figure 11. Summary plots of Xpert MTB/RIF sensitivity and specificity for detection of pulmonary tuberculosis. Each individual study is represented by an empty square. The size of the square is proportional to the sample size of the study such that larger studies are represented by larger squares. The filled circle is the median pooled estimate for sensitivity and specificity. The solid curves represent the $95 \%$ credible region around the summary estimate; the dashed curves represent the $95 \%$ prediction region.

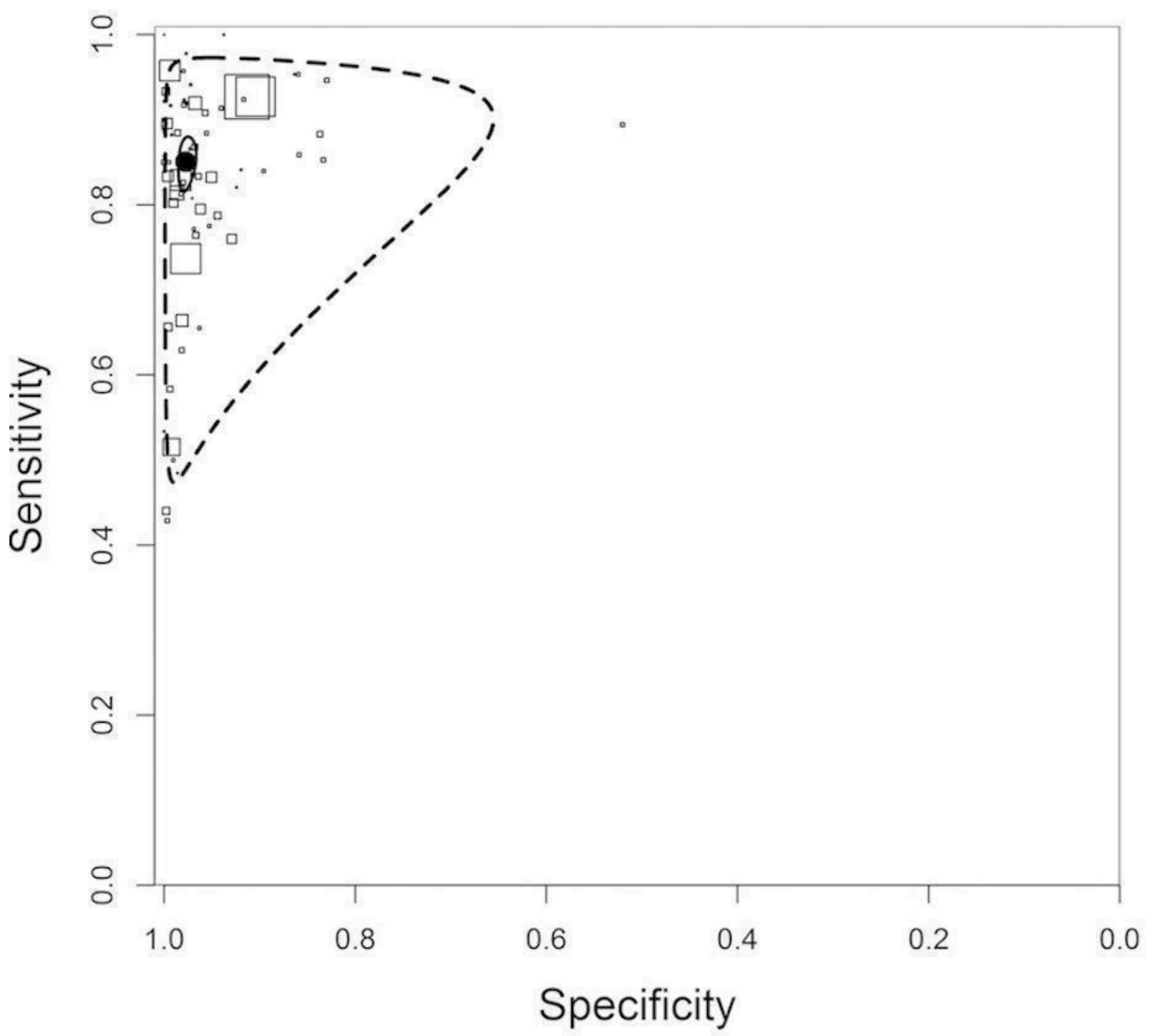

\section{A.2. Xpert Ultra}

We identified one study that evaluated Xpert Ultra for PTB (Dorman 2018). This multicentre study, which took place in Belarus, Brazil, China, Georgia, India, Kenya, South Africa, and Uganda, compared Xpert Ultra and Xpert MTB/RIF on the same participant specimens,
(1439 participants). Based on a reference standard of multiple cultures, Xpert Ultra yielded higher sensitivity at $88 \%$ (95\% Cl $85 \%$ to $91 \%$ ), compared to Xpert MTB/RIF sensitivity of $83 \%$ (79\% to $86 \%$ ), and lower specificity at $96 \%$ (94\% to $97 \%)$, compared to Xpert MTB/ RIF specificity of $98 \%$ ( $97 \%$ to $99 \%$ ) (Figure 12 ). 
Figure 12. Summary ROC plots for sensitivity and specificity of Xpert MTB/RIF and Xpert Ultra for detection of pulmonary tuberculosis.

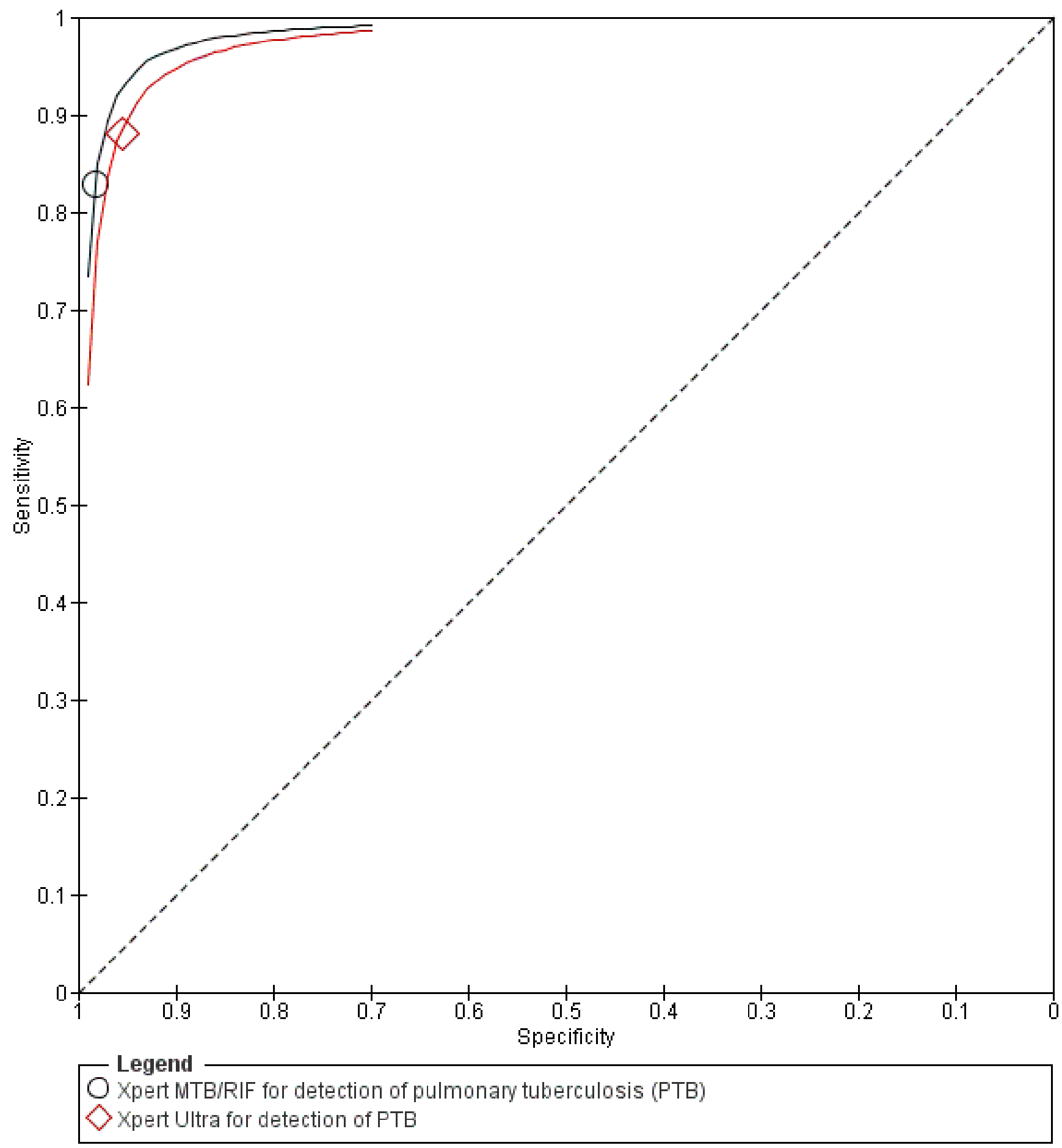

\section{B. Investigations of heterogeneity}

Unless otherwise noted, investigations of heterogeneity are limited to those studies that enrolled unselected participants.

\section{B.1. Xpert MTB/RIF for detection of PTB by smear status}

B.1.a. Xpert MTB/RIF accuracy in participants with smear-positive sputum specimens

Figure 13 displays the forest plots for studies reporting data for participants with smear-positive specimens. Sensitivity estimates

Xpert MTB/RIF and Xpert MTB/RIF Ultra for pulmonary tuberculosis and rifampicin resistance in adults (Review) 35

Copyright ( 2019 The Authors. Cochrane Database of Systematic Reviews published by John Wiley \& Sons, Ltd. on behalf of The Cochrane Collaboration. 
ranged from $75 \%$ to $100 \%$ and specificity estimates from $0 \%$ to $100 \%$. We thought some of the variability in specificity estimates could be explained by small numbers of participants included in the studies. In addition, in some studies, including the four largest, the value for true negatives was zero (tuberculosis was not present when measured against culture), and all participants were considered to be true positives (tuberculosis was present when measured against culture). 
Figure 13. Forest plots of Xpert MTB/RIF sensitivity and specificity for detection of pulmonary tuberculosis, participants with smear-positive (culture-positive) specimens. The individual studies are ordered by decreasing sensitivity. The squares represent the sensitivity and specificity of one study, the black line its confidence interval. FN: false-negative; FP: false-positive; TN: true-negative; TP: true-positive.

\section{Study}

Balcells 2012

Carriquiry 2012

Chaisson 2014

Chen 2017

Chew 2016

Davis 2014

Hanrahan 2013

Helb 2010

Kawkitinarong 2017

Ko 2016

Kurbatova 2013

Lawn 2011

Le Palud 2014

Lippincott 2014

Meawed 2016

Mok 2016

Moussa 2016

O'Donnell 2015

Safianowska 2012

Theron 2013

Milliamson 2012

Zeka 2011

Zmak 2013

Sharma 2015

Nikam 2014

Dorman 2018

Shenai 2016

Luetkemeyer 2016

Boehme 2011

Boehme 2010

Rachow 2011

Pimkina 2015

Hanif 2011

Rice 2017

Reddy 2017

Metcalfe 2015

Huang 2015

Balcha 2014

Huh 2014

Scott 2011

Hanrahan 2014

Geleta 2015

Tsuyuguchi 2017

Theron 2011

Shao 2017

Ngabonziza 2016

Cowan 2017

Reechaipichitkul 2017

Kwak 2013

Sohn 2014

Park 2013

Sah 2017

Van Rie 2013
TP FP FN TN Sensitivity $(95 \% \mathrm{Cl})$ Specificity $(95 \% \mathrm{Cl})$

$\begin{array}{llllll}8 & 0 & 0 & 2 & 1.00[0.63,1.00] & 1.00[0.16,1.00]\end{array}$

$\begin{array}{llllll}31 & 1 & 0 & 2 & 1.00[0.89,1.00] & 0.67[0.09,0.99]\end{array}$

$\begin{array}{llllll}8 & 0 & 0 & 1 & 1.00[0.63,1.00] & 1.00[0.03,1.00]\end{array}$

$\begin{array}{lllll}3 & 0 & 0 & 2 & 1.00[0.29,1.00]\end{array}$

$\begin{array}{lllll}16 & 0 & 0 & 0 & 1.00[0.79,1.00]\end{array}$

$\begin{array}{lllll}11 & 3 & 0 & 8 & 1.00[0.72,1.00]\end{array}$

$\begin{array}{lllll}15 & 0 & 0 & 1 & 1.00[0.78,1.00]\end{array}$

$\begin{array}{lllll}29 & 0 & 0 & 0 & 1.00[0.88,1.00]\end{array}$

$\begin{array}{lllll}128 & 3 & 0 & 3 & 1.00[0.97,1.00]\end{array}$

$\begin{array}{lllll}33 & 0 & 0 & 0 & 1.00[0.89,1.00]\end{array}$

$\begin{array}{lllll}91 & 0 & 0 & 0 & 1.00[0.96,1.00]\end{array}$

$\begin{array}{lllll}19 & 0 & 0 & 0 & 1.00[0.82,1.00]\end{array}$

$\begin{array}{lllll}5 & 0 & 0 & 6 & 1.00[0.48,1.00]\end{array}$

$\begin{array}{lllll}12 & 0 & 0 & 5 & 1.00[0.74,1.00]\end{array}$

$49 \quad 0 \quad 0 \quad 0 \quad 1.00[0.93,1.00]$

$\begin{array}{lllll}9 & 0 & 0 & 0 & 1.00[0.66,1.00]\end{array}$

$\begin{array}{lllll}61 & 0 & 0 & 0 & 1.00[0.94,1.00]\end{array}$

$\begin{array}{lllll}91 & 1 & 0 & 0 & 1.00[0.96,1.00]\end{array}$

$\begin{array}{lllll}12 & 1 & 0 & 7 & 1.00[0.74,1.00]\end{array}$

$\begin{array}{lllll}16 & 1 & 0 & 0 & 1.00[0.79,1.00]\end{array}$

$\begin{array}{lllll}67 & 0 & 0 & 22 & 1.00[0.95,1.00]\end{array}$

$24 \quad 0 \quad 0 \quad 0 \quad 1.00[0.86,1.00]$

$\begin{array}{rrrrr}3 & 0 & 0 & 0 & 1.00[0.29,1.00]\end{array}$

$\begin{array}{lllll}374 & 0 & 3 & 0 & 0.99[0.98,1.00]\end{array}$

$\begin{array}{lllll}92 & 13 & 1 & 2 & 0.99[0.94,1.00]\end{array}$

$\begin{array}{lllll}319 & 0 & 4 & 0 & 0.99[0.97,1.00]\end{array}$

$\begin{array}{lllll}74 & 0 & 1 & 0 & 0.99[0.93,1.00]\end{array}$

$\begin{array}{lllll}129 & 1 & 2 & 17 & 0.98[0.95,1.00]\end{array}$

$\begin{array}{lllll}637 & 0 & 11 & 0 & 0.98[0.97,0.99]\end{array}$

$\begin{array}{lllll}551 & 0 & 10 & 0 & 0.98[0.97,0.99]\end{array}$

$\begin{array}{lllll}50 & 0 & 1 & 0 & 0.98[0.90,1.00]\end{array}$

$\begin{array}{lllll}273 & 1 & 6 & 2 & 0.98[0.95,0.99]\end{array}$

$\begin{array}{lllll}45 & 0 & 1 & 0 & 0.98[0.88,1.00]\end{array}$

$\begin{array}{lllll}85 & 0 & 2 & 124 & 0.98 \\ 8 & 0.92,1.00]\end{array}$

$\begin{array}{lllll}41 & 0 & 1 & 2 & 0.98[0.87,1.00]\end{array}$

$\begin{array}{lllll}79 & 0 & 2 & 0 & 0.98[0.91,1.00]\end{array}$

$\begin{array}{lllll}88 & 0 & 3 & 5 & 0.97 \\ 20.91,0.99]\end{array}$

$\begin{array}{lllll}27 & 3 & 1 & 0 & 0.96[0.82,1.00]\end{array}$

$\begin{array}{lllll}76 & 3 & 3 & 18 & 0.96[0.89,0.99]\end{array}$

$\begin{array}{lllll}47 & 0 & 2 & 0 & 0.96[0.86,1.00]\end{array}$

$\begin{array}{lllll}178 & 3 & 8 & 7 & 0.96[0.92,0.98]\end{array}$

$\begin{array}{lllll}20 & 0 & 1 & 1 & 0.95[0.76,1.00]\end{array}$

$\begin{array}{llll}180 & 4 & 9 & 32\end{array}$

$0.95[0.91,0.98]$

$0.95[0.88,0.98]$

$0.94[0.87,0.98]$

$0.94[0.83,0.99]$

$0.94[0.70,1.00]$

$0.90[0.73,0.98]$

$0.89[0.78,0.95]$

$0.86[0.42,1.00]$

$0.83[0.59,0.96]$

$0.79[0.59,0.92]$

$0.75[0.19,0.99]$
Not estimable

$0.73[0.39,0.94]$

$1.00[0.03,1.00]$

Not estimable

$0.50[0.12,0.88]$

Not estimable

Not estimable

Not estimable

$1.00[0.54,1.00]$

$1.00[0.48,1.00]$

Not estimable

Not estimable

Not estimable

$0.00[0.00,0.97]$

$0.88[0.47,1.00]$

$0.00[0.00,0.97]$

$1.00[0.85,1.00]$

Not estimable

Not estimable

Not estimable

$0.13[0.02,0.40]$

Not estimable

Not estimable

$0.94[0.73,1.00]$

Not estimable

Not estimable

Not estimable

$0.67[0.09,0.99]$

Not estimable

$1.00[0.97,1.00]$

$1.00[0.16,1.00]$

Not estimable

$1.00[0.48,1.00]$

$0.00[0.00,0.71]$

$0.86[0.64,0.97]$

Not estimable

$0.70[0.35,0.93]$

$1.00[0.03,1.00]$

$0.89[0.74,0.97]$

Not estimable

$0.80[0.28,0.99]$

$1.00[0.03,1.00]$

$1.00[0.59,1.00]$

$0.75[0.19,0.99]$

$1.00[0.79,1.00]$

$1.00[0.40,1.00]$

$1.00[0.83,1.00]$

$0.33[0.01,0.91]$

$1.00[0.16,1.00]$
$1.00[0.16,1.00]$
Sensitivity $(95 \% \mathrm{Cl}) \quad$ Specificity $(95 \% \mathrm{CI})$

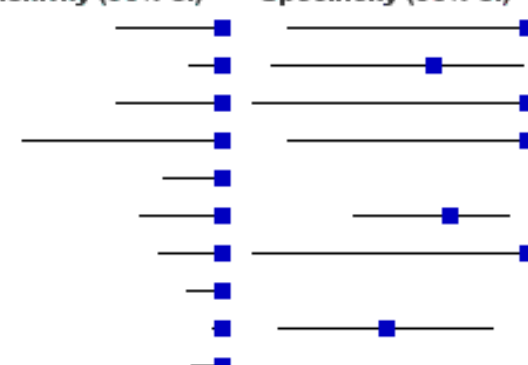

$\rightarrow$

$\rightarrow$
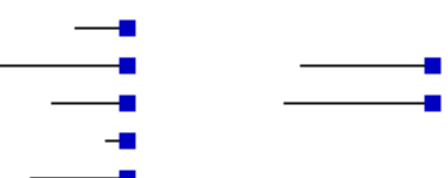

$-1$

$-$
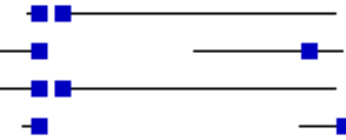

$-$

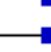

$\longrightarrow$

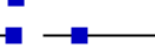

-

-

-

$-$
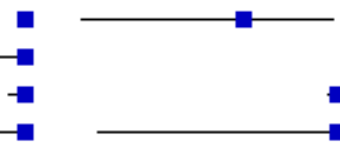

$\rightarrow$

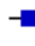

$-7$

$\rightarrow$

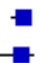
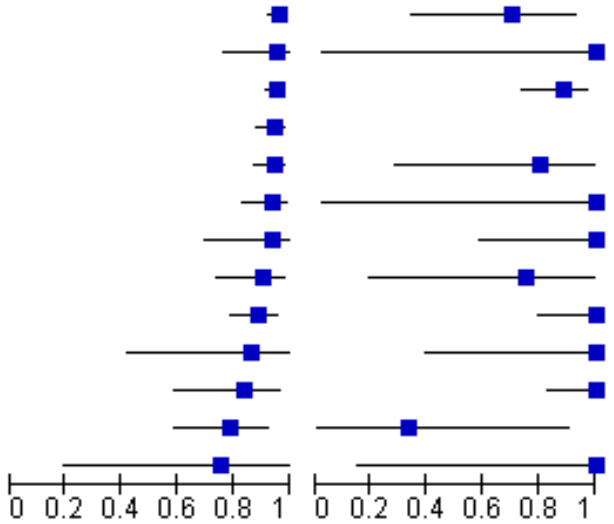
For smear-positive, culture-positive PTB, using a univariate random-effects model and including all studies for which sensitivity data were available, Xpert MTB/RIF pooled sensitivity ( $95 \% \mathrm{Crl}$ ) was $98 \%$ ( $97 \%$ to $99 \%$ ) (53 studies, 4574 participants). We did not determine pooled specificity because in many studies the value for true negatives was zero.

\section{B.1.b. Xpert MTB/RIF accuracy in participants with smear-negative} sputum specimens

Figure 14 displays the forest plots for studies reporting data for participants with smear-negative specimens. Sensitivity estimates ranged from $28 \%$ to $100 \%$. The lowest sensitivity was described by Sohn 2014; this study evaluated induced sputum specimens from participants with presumptive PTB, most of whom were asymptomatic. Specificity estimates ranged from $57 \%$ to $100 \%$. The lowest specificity was described by Nikam 2014, with the remaining 55 studies ranging in specificity from $83 \%$ to $100 \%$. 
Figure 14. Forest plots of Xpert MTB/RIF sensitivity and specificity for detection of pulmonary tuberculosis, participants with smear-negative (culture-positive) specimens. The individual studies are ordered by decreasing 
sensitivity. The squares represent the sensitivity and specificity of one study, the black line its confidence interval. FN: false-negative; FP: false-positive; TN: true-negative; TP: true-positive.

\begin{tabular}{|c|c|c|c|c|c|c|c|c|}
\hline Study & TP & FP & FN & TN & Sensitivity (95\% Cl) & Specificity (95\% Cl) & Sensitivity (95\% Cl) & Specificity (95\% Cl) \\
\hline Meawed 2016 & 5 & 1 & 0 & 3 & $1.00[0.48,1.00]$ & $0.75[0.19,0.99]$ & & \\
\hline Shao 2017 & 24 & 30 & 1 & 147 & $0.96[0.80,1.00]$ & $0.83[0.77,0.88]$ & & $\rightarrow$ \\
\hline Carriquiry 2012 & 13 & 1 & 1 & 82 & $0.93[0.66,1.00]$ & $0.99[0.93,1.00]$ & $\rightarrow$ & $\rightarrow$ \\
\hline Sah 2017 & 10 & 3 & 1 & 60 & $0.91[0.59,1.00]$ & $0.95[0.87,0.99]$ & $\rightarrow-$ & $\rightarrow$ \\
\hline Ko 2016 & 64 & 12 & 8 & 132 & $0.89[0.79,0.95]$ & $0.92[0.86,0.96]$ & $\rightarrow$ & $\rightarrow$ \\
\hline Kawkitinarong 2017 & 103 & 3 & 19 & 130 & $0.84[0.77,0.90]$ & $0.98[0.94,1.00]$ & $\rightarrow$ & 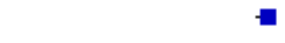 \\
\hline Pimkina 2015 & 85 & 32 & 18 & 375 & $0.83[0.74,0.89]$ & $0.92[0.89,0.95]$ & $\rightarrow$ & - \\
\hline Theron 2013 & 9 & 4 & 2 & 120 & $0.82[0.48,0.98]$ & $0.97[0.92,0.99]$ & $\rightarrow-$ & $\rightarrow$ \\
\hline Huang 2015 & 78 & 31 & 19 & 154 & $0.80[0.71,0.88]$ & $0.83[0.77,0.88]$ & $\rightarrow$ & $\rightarrow$ \\
\hline Park 2013 & 4 & 6 & 1 & 271 & $0.80[0.28,0.99]$ & $0.98[0.95,0.99]$ & - & घ \\
\hline Reechaipichitkul 2017 & 26 & 4 & 7 & 54 & $0.79[0.61,0.91]$ & $0.93[0.83,0.98]$ & $\longrightarrow-$ & $\rightarrow$ \\
\hline Moure 2011 & 61 & 0 & 17 & 29 & $0.78[0.67,0.87]$ & $1.00[0.88,1.00]$ & $\rightarrow$ & $\rightarrow$ \\
\hline Sharma 2015 & 56 & 6 & 16 & 984 & $0.78[0.66,0.87]$ & $0.99[0.99,1.00]$ & & घ \\
\hline Boehme 2011 & 296 & 30 & 89 & 2846 & $0.77[0.72,0.81]$ & $0.99[0.99,0.99]$ & & - \\
\hline Huh 2014 & 19 & 7 & 6 & 139 & $0.76[0.55,0.91]$ & $0.95[0.90,0.98]$ & & 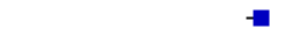 \\
\hline Balcells 2012 & 3 & 1 & 1 & 145 & $0.75[0.19,0.99]$ & $0.99[0.96,1.00]$ & & - \\
\hline Chew 2016 & 18 & 1 & 6 & 197 & $0.75[0.53,0.90]$ & $0.99[0.97,1.00]$ & & 口 \\
\hline O'Donnell 2015 & 21 & 1 & 7 & 34 & $0.75[0.55,0.89]$ & $0.97[0.85,1.00]$ & & $\rightarrow$ \\
\hline Zmak 2013 & 3 & 0 & 1 & 110 & $0.75[0.19,0.99]$ & $1.00[0.97,1.00]$ & & - \\
\hline Rice 2017 & 35 & 2 & 12 & 476 & $0.74[0.60,0.86]$ & $1.00[0.98,1.00]$ & $\rightarrow-$ & घ \\
\hline Nikam 2014 & 43 & 46 & 15 & 62 & $0.74[0.61,0.85]$ & $0.57[0.48,0.67]$ & & $\rightarrow-$ \\
\hline Le Palud 2014 & 11 & 2 & 4 & 134 & $0.73[0.45,0.92]$ & $0.99[0.95,1.00]$ & & $=$ \\
\hline Kwak 2013 & 68 & 20 & 25 & 489 & $0.73[0.63,0.82]$ & $0.96[0.94,0.98]$ & $=$ & - \\
\hline Boehme 2010 & 124 & 5 & 47 & 604 & $0.73[0.65,0.79]$ & $0.99[0.98,1.00]$ & $\rightarrow$ & घ \\
\hline Helb 2010 & 38 & 0 & 15 & 25 & $0.72[0.58,0.83]$ & $1.00[0.86,1.00]$ & & $\rightarrow$ \\
\hline Kurbatova 2013 & 11 & 17 & 5 & 104 & $0.69[0.41,0.89]$ & $0.86[0.78,0.92]$ & & \\
\hline Shenai 2016 & 15 & 5 & 7 & 234 & $0.68[0.45,0.86]$ & $0.98[0.95,0.99]$ & & - \\
\hline Chen 2017 & 2 & 3 & 1 & 722 & $0.67[0.09,0.99]$ & $1.00[0.99,1.00]$ & & घ \\
\hline Moussa 2016 & 6 & 3 & 3 & 145 & $0.67[0.30,0.93]$ & $0.98[0.94,1.00]$ & & - \\
\hline Reddy 2017 & 37 & 12 & 19 & 250 & $0.66[0.52,0.78]$ & $0.95[0.92,0.98]$ & & - \\
\hline Ngabonziza 2016 & 31 & 5 & 16 & 498 & $0.66[0.51,0.79]$ & $0.99[0.98,1.00]$ & & घ \\
\hline Hanif 2011 & 9 & 0 & 5 & 146 & $0.64[0.35,0.87]$ & $1.00[0.98,1.00]$ & & - \\
\hline Van Rie 2013 & 7 & 1 & 4 & 142 & $0.64[0.31,0.89]$ & $0.99[0.96,1.00]$ & & ㅂ. \\
\hline Zeka 2011 & 7 & 0 & 4 & 68 & $0.64[0.31,0.89]$ & $1.00[0.95,1.00]$ & & $\rightarrow$ \\
\hline Tadesse 2016 & 12 & 2 & 7 & 164 & $0.63[0.38,0.84]$ & $0.99[0.96,1.00]$ & & - \\
\hline Rachow 2011 & 11 & 1 & 7 & 102 & $0.61[0.36,0.83]$ & $0.99[0.95,1.00]$ & & 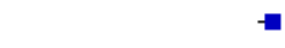 \\
\hline Scott 2011 & 11 & 3 & 7 & 107 & $0.61[0.36,0.83]$ & $0.97[0.92,0.99]$ & & $\rightarrow$ \\
\hline Mok 2016 & 21 & 2 & 14 & 112 & $0.60[0.42,0.76]$ & $0.98[0.94,1.00]$ & & $\rightarrow$ \\
\hline Safianowska 2012 & 3 & 0 & 2 & 120 & $0.60[0.15,0.95]$ & $1.00[0.97,1.00]$ & & = \\
\hline Balcha 2014 & 54 & 10 & 40 & 675 & $0.57[0.47,0.68]$ & $0.99[0.97,0.99]$ & $\rightarrow-$ & घ \\
\hline Luetkemeyer 2016 & 46 & 9 & 38 & 718 & $0.55[0.44,0.66]$ & $0.99[0.98,0.99]$ & $\rightarrow-$ & घ \\
\hline Hanrahan 2013 & 26 & 2 & 22 & 478 & $0.54[0.39,0.69]$ & $1.00[0.99,1.00]$ & $\longrightarrow-$ & - \\
\hline Meyer 2017 & 207 & 68 & 183 & 1324 & $0.53[0.48,0.58]$ & $0.95[0.94,0.96]$ & $=$ & - \\
\hline Hanrahan 2014 & 97 & 32 & 91 & 1440 & $0.52[0.44,0.59]$ & $0.98[0.97,0.99]$ & - & - \\
\hline Cowan 2017 & 2 & 0 & 2 & 291 & $0.50[0.07,0.93]$ & $1.00[0.99,1.00]$ & & - \\
\hline Davis 2014 & 1 & 0 & 1 & 132 & $0.50[0.01,0.99]$ & $1.00[0.97,1.00]$ & & $\square$ \\
\hline Lippincott 2014 & 1 & 1 & 1 & 479 & $0.50[0.01,0.99]$ & $1.00[0.99,1.00]$ & & \\
\hline Walusimbi 2013a & 21 & 16 & 22 & 310 & $0.49[0.33,0.65]$ & $0.95[0.92,0.97]$ & $\longrightarrow$ & - \\
\hline Geleta 2015 & 18 & 6 & 19 & 155 & $0.49[0.32,0.66]$ & $0.96[0.92,0.99]$ & $\longrightarrow$ & 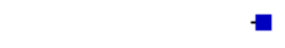 \\
\hline Theron 2011 & 22 & 19 & 25 & 320 & $0.47[0.32,0.62]$ & $0.94[0.91,0.97]$ & $\longrightarrow-$ & - \\
\hline Dorman 2018 & 63 & 17 & 74 & 957 & $0.46[0.37,0.55]$ & $0.98[0.97,0.99]$ & $\rightarrow-$ & घ \\
\hline Tsuyuguchi 2017 & 17 & 2 & 21 & 148 & $0.45[0.29,0.62]$ & $0.99[0.95,1.00]$ & $\longrightarrow$ & $=$ \\
\hline Lawn 2011 & 23 & 2 & 30 & 320 & $0.43[0.30,0.58]$ & $0.99[0.98,1.00]$ & $\rightarrow-$ & - \\
\hline Metcalfe 2015 & 3 & 8 & 5 & 52 & $0.38[0.09,0.76]$ & $0.87[0.75,0.94]$ & & \\
\hline Sohn 2014 & 5 & 1 & 13 & 406 & $0.28[0.10,0.53]$ & $1.00[0.99,1.00]$ & & घ \\
\hline Chaisson 2014 & 0 & 0 & 1 & 132 & $0.00[0.00,0.97]$ & $1.00[0.97,1.00]$ & & $\Rightarrow$ \\
\hline
\end{tabular}


For smear-negative, culture-positive PTB, using a bivariate model and including all studies for which sensitivity and specificity data were available, Xpert MTB/RIF pooled sensitivity and specificity (95\% Crl) were $67 \%$ (63\% to $72 \%$ ) and $98 \%$ (97\% to 99\%), (56 studies, 22,581 participants).

B.1.C. Xpert MTB/RIF accuracy by smear status, studies that provided data for both smear-positive and smear-negative participants

We limited this analysis to 45 studies that reported results for participants with smear-positive specimens and smear-negative specimens within the same study (Balcells 2012; Balcha 2014; Boehme 2010; Boehme 2011; Carriquiry 2012; Chaisson 2014; Chen 2017; Chew 2016; Cowan 2017; Davis 2014; Dorman 2018; Geleta 2015; Hanif 2011; Hanrahan 2013; Hanrahan 2014; Helb 2010; Huang 2015; Huh 2014; Kawkitinarong 2017; Ko 2016; Kurbatova 2013; Kwak 2013; Lawn 2011; Lippincott 2014; Luetkemeyer 2016; Moussa 2016 Ngabonziza 2016; Nikam 2014; O'Donnell 2015; Park 2013; Rachow 2011; Reddy 2017; Reechaipichitkul 2017; Rice 2017; Safi- anowska 2012; Sah 2017; Scott 2011; Shao 2017; Sharma 2015; Shenai 2016; Sohn 2014; Theron 2011; Tsuyuguchi 2017; Zeka 2011; Zmak 2013). For smear-positive tuberculosis, Xpert MTB/RIF pooled sensitivity was $98 \%$ ( $97 \%$ to $98 \%$ ), considerably higher than the sensitivity of $68 \%$ (63\% to $73 \%$ ) for smear-negative tuberculosis (Table 2).

B.1.d. Xpert MTB/RIF versus Xpert Ultra for detection of PTB by smear status, direct comparison

One study compared Xpert Ultra and Xpert MTB/RIF for detection of PTB by smear status against a reference standard of multiple cultures (Dorman 2018). In smear-positive participants, sensitivities $(95 \% \mathrm{Cl})$ of Xpert Ultra and Xpert MTB/RIF were identical at $99 \%$ (97\% to $100 \%$ ) (323 participants). In smear-negative participants, Xpert Ultra yielded higher sensitivity at $63 \%(95 \% \mathrm{Cl} 54 \%$ to $71 \%)$, compared to Xpert MTB/RIF sensitivity of $46 \%$ ( $37 \%$ to $55 \%$ ), and lower specificity at $96 \%$ (94\% to $97 \%$ ), compared to Xpert MTB/RIF specificity of $98 \%$ (97\% to $99 \%$ ) (Figure 15$)$.

Figure 15. Forest plots comparing Xpert MTB/RIF and Xpert Ultra sensitivity and specificity for detection of pulmonary tuberculosis in smear-positive and smear-negative participants. The individual studies are ordered by decreasing sensitivity. The squares represent the sensitivity and specificity of one study, the black line its $\mathrm{Cl}$. TP = true positive; $F P=$ false positive; $F N=$ false negative; $T N=$ true negative.

Smear-positive, Xpert MTB/RIF, direct comparison Xpert MTB/RIF vs Xpert Ultra

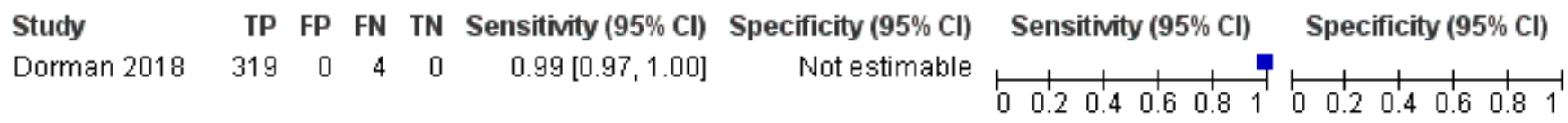

Smear-positive, Xpert Ultra, direct comparison Xpert MTB/RIF vs Xpert Ultra

\begin{tabular}{|c|c|c|c|c|c|c|c|c|}
\hline Stucly & TP & FP & FN & TN & Sensitivity (95\% Cl) & Specificity (95\% Cl) & Sensitivity (95\% Cl) & Specificity $(95 \% \mathrm{Cl})$ \\
\hline Dorman 2018 & 322 & 0 & 1 & 0 & $1.00[0.98,1.00]$ & Not estimable & & \\
\hline
\end{tabular}

Smear-negative, Xpert MTB/RIF, direct comparison Xpert MTB/RIF vs Xpert Ultra

\begin{tabular}{|c|c|c|c|c|c|c|c|c|}
\hline Study & TP & FP & FN & TN & Sensitivity (95\% Cl) & Specificity (95\% Cl) & Sensitivity (95\% Cl) & Specificity ( $95 \%$ Cl) \\
\hline Dorman 2018 & 63 & 17 & 74 & 957 & $0.46[0.37,0.55]$ & $0.98[0.97,0.99]$ &,+ & \\
\hline
\end{tabular}

Smear-negative, Xpert Ultra, direct comparison Xpert MTB/RIF vs Xpert Ultra

\begin{tabular}{|c|c|c|c|c|c|c|c|c|}
\hline Study & TP & FP & FN & TN & Sensitivity (95\% Cl) & Specificity (95\% Cl) & Sensitivity (95\% Cl) & Specificity ( $95 \% \mathrm{Cl})$ \\
\hline Dorman 2018 & 86 & 43 & 51 & 931 & $0.63[0.54,0.71]$ & $0.96[0.94,0.97]$ & $1, \frac{7}{7}$ & \\
\hline
\end{tabular}

\section{B.2. Xpert MTB/RIF for detection of PTB by HIV status}

\section{B.2.a. Xpert MTB/RIF accuracy in HIV-negative people}

In HIV-negative participants, Xpert MTB/RIF sensitivity estimates ranged from $56 \%$ to $100 \%$ and specificity estimates from $95 \%$ to
$100 \%$ (Figure 16). We included all studies that provided data in this analysis. In HIV-negative participants, Xpert MTB/RIF pooled sensitivity and specificity $(95 \% \mathrm{Crl})$ were $89 \%(85 \%$ to $92 \%)$ and $98 \%$ (97\% to $99 \%)$, (18 studies, 5118 participants). 
Figure 16. Forest plots of Xpert MTB/RIF sensitivity and specificity for detection of pulmonary tuberculosis in HIV-negative participants. The individual studies are ordered by decreasing sensitivity. The squares represent the sensitivity and specificity of one study, the black line its confidence interval. FN: false-negative; FP: false-positive; TN: true-negative; TP: true-positive.

$\begin{array}{lrrrrr}\text { Study } & \text { TP } & \text { FP } & \text { FN } & \text { TN } & \text { Sensitivity }(95 \% \text { Cl) } \\ \text { Theron 2013 } & 12 & 3 & 0 & 69 & 1.00[0.74,1.00] \\ \text { Bates 2013a } & 3 & 0 & 0 & 19 & 1.00[0.29,1.00] \\ \text { Calligaro 2015 } & 5 & 4 & 0 & 39 & 1.00[0.48,1.00] \\ \text { Moussa 2016 } & 67 & 3 & 3 & 145 & 0.96[0.88,0.99] \\ \text { Boum 2016 } & 72 & 7 & 5 & 190 & 0.94[0.85,0.98] \\ \text { Pinyopornpanish 2015 } & 28 & 2 & 2 & 41 & 0.93[0.78,0.99] \\ \text { Boehme 2010 } & 335 & 1 & 26 & 173 & 0.93[0.90,0.95] \\ \text { Boehme 2011 } & 304 & 5 & 31 & 748 & 0.91[0.87,0.94] \\ \text { Dorman 2018 } & 143 & 9 & 16 & 315 & 0.90[0.84,0.94] \\ \text { Rachow 2011 } & 17 & 0 & 2 & 53 & 0.89[0.67,0.99] \\ \text { Safianowska 2012 } & 15 & 1 & 2 & 127 & 0.88[0.64,0.99] \\ \text { Luetkemeyer 2016 } & 111 & 4 & 17 & 396 & 0.87[0.80,0.92] \\ \text { Scott 2011 } & 12 & 0 & 2 & 17 & 0.86[0.57,0.98] \\ \text { Theron 2011 } & 68 & 9 & 14 & 195 & 0.83[0.73,0.90] \\ \text { Hanrahan 2014 } & 120 & 13 & 29 & 689 & 0.81[0.73,0.87] \\ \text { Calligaro 2017 } & 10 & 2 & 3 & 138 & 0.77[0.46,0.95] \\ \text { Van Rie 2013 } & 2 & 0 & 1 & 33 & 0.67[0.09,0.99] \\ \text { Hanrahan 2013 } & 5 & 0 & 4 & 182 & 0.56[0.21,0.86]\end{array}$

B.2.b. Xpert MTB/RIF accuracy in HIV-positive people

In HIV-positive participants, Xpert MTB/RIF sensitivity estimates ranged from $67 \%$ to $100 \%$ and specificity estimates from $92 \%$ to

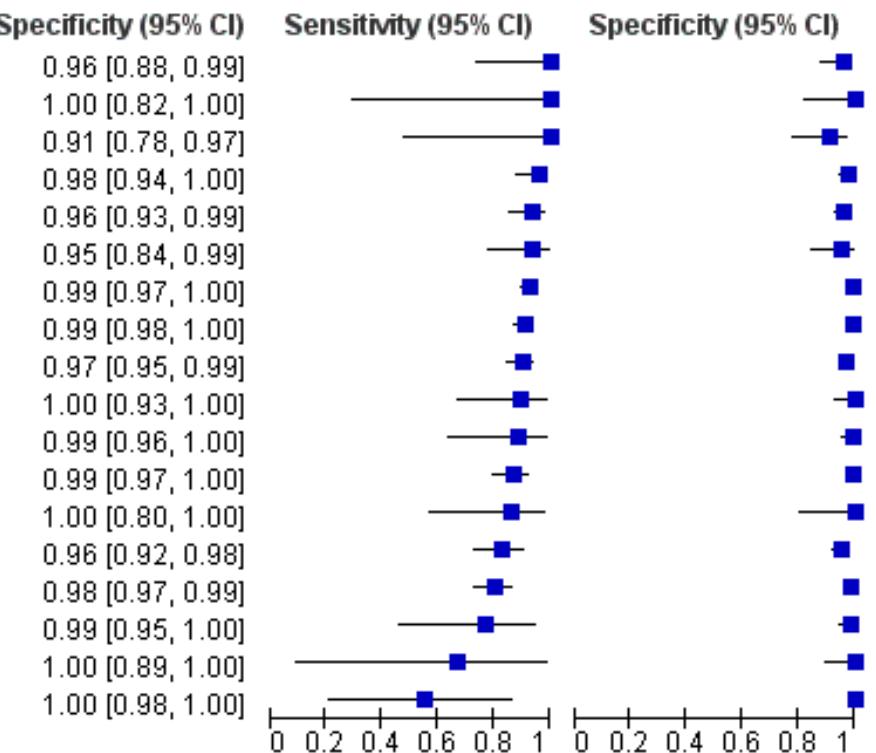

$100 \%$ (Figure 17). We included all studies that provided data in this analysis. In HIV-positive participants, Xpert MTB/RIF pooled sensitivity and specificity $(95 \% \mathrm{Crl})$ were $77 \%(71 \%$ to $82 \%)$ and $98 \%$ (98\% to 99\%), (30 studies, 9589 participants). 
Figure 17. Forest plots of Xpert MTB/RIF sensitivity and specificity for detection of pulmonary tuberculosis in HIV-positive participants. The individual studies are ordered by decreasing sensitivity. The squares represent the sensitivity and specificity of one study, the black line its confidence interval. FN: false-negative; FP: false-positive; TN: true-negative; TP: true-positive.

$\begin{array}{lrrrrr}\text { Stucly } & \text { TP } & \text { FP } & \text { FN } & \text { TN } & \text { Sensitivity }(95 \% \text { CI) } \\ \text { Calligaro 2015 } & 3 & 0 & 0 & 22 & 1.00[0.29,1.00] \\ \text { Mollel 2017 } & 9 & 0 & 0 & 60 & 1.00[0.66,1.00] \\ \text { Pinyopornpanish 2015 } & 10 & 0 & 0 & 16 & 1.00[0.69,1.00] \\ \text { Carriquiry 2012 } & 44 & 2 & 1 & 84 & 0.98[0.88,1.00] \\ \text { O'Donnell 2015 } & 49 & 1 & 4 & 26 & 0.92[0.82,0.98] \\ \text { Balcells 2012 } & 11 & 1 & 1 & 147 & 0.92[0.62,1.00] \\ \text { Boum 2016 } & 22 & 15 & 12 & 464 & 0.91[0.85,0.95] \\ \text { Boehme 2010 } & 97 & 2 & 14 & 225 & 0.87[0.80,0.93] \\ \text { Scott 2011 } & 45 & 3 & 7 & 84 & 0.87[0.74,0.94] \\ \text { Calligaro 2017 } & 25 & 11 & 4 & 204 & 0.86[0.68,0.96] \\ \text { Boehme 2011 } & 173 & 3 & 37 & 382 & 0.82[0.77,0.87] \\ \text { Rachow 2011 } & 41 & 1 & 9 & 49 & 0.82[0.69,0.91] \\ \text { Bates 2013a } & 17 & 2 & 4 & 39 & 0.81[0.58,0.95] \\ \text { Bjerrum 2016 } & 27 & 5 & 8 & 155 & 0.77[0.60,0.90] \\ \text { Dorman 2018 } & 88 & 2 & 27 & 315 & 0.77[0.68,0.84] \\ \text { Ssengooba 2014 } & 94 & 10 & 29 & 291 & 0.76[0.68,0.84] \\ \text { Theron 2013 } & 6 & 2 & 2 & 34 & 0.75[0.35,0.97] \\ \text { Luetkemeyer 2016 } & 64 & 6 & 23 & 339 & 0.74[0.63,0.82] \\ \text { Theron 2011 } & 32 & 7 & 14 & 77 & 0.70[0.54,0.82] \\ \text { Hanrahan 2014 } & 169 & 23 & 74 & 887 & 0.70[0.63,0.75] \\ \text { Hanrahan 2013 } & 36 & 2 & 16 & 325 & 0.69[0.55,0.81] \\ \text { Van Rie 2013 } & 8 & 1 & 4 & 99 & 0.67[0.35,0.90] \\ \text { Balcha 2014 } & 81 & 13 & 41 & 677 & 0.66[0.57,0.75] \\ \text { Henostroza 2016 } & 39 & 5 & 23 & 266 & 0.63[0.50,0.75] \\ \text { Lawn 2011 } & 42 & 2 & 30 & 320 & 0.58[0.46,0.70] \\ \text { Al-Darraji 2013 } & 8 & 0 & 7 & 110 & 0.53[0.27,0.79] \\ \text { Yoon 2017 } & 84 & 8 & 79 & 1006 & 0.52[0.44,0.59] \\ \text { Adelman 2015 } & 3 & 2 & 3 & 204 & 0.50[0.12,0.88] \\ \text { Walusimbi 2013a } & 21 & 16 & 22 & 310 & 0.49[0.33,0.65] \\ \text { LaCourse 2016 } & 3 & 1 & 4 & 280 & 0.43[0.10,0.82]\end{array}$

B.2.c. Xpert MTB/RIF accuracy by HIV status, studies that provided data for both HIV-negative and HIV-positive individuals

We limited this analysis to 14 studies that reported results for HIV-negative and HIV-positive participants within the same study (Bates 2013a; Boum 2016; Boehme 2010; Boehme 2011; Calligaro 2015; Calligaro 2017; Dorman 2018; Hanrahan 2013; Hanrahan 2014; Luetkemeyer 2016; Pinyopornpanish 2015; Rachow 2011;

\author{
Specificity $(95 \% \mathrm{Cl})$ \\ $1.00[0.85,1.00]$ \\ $1.00[0.94,1.00]$ \\ $1.00[0.79,1.00]$ \\ $0.98[0.92,1.00]$ \\ $0.96[0.81,1.00]$ \\ $0.99[0.96,1.00]$ \\ $0.97[0.95,0.98]$ \\ $0.99[0.97,1.00]$ \\ $0.97[0.90,0.99]$ \\ $0.95[0.91,0.97]$ \\ $0.99[0.98,1.00]$ \\ $0.98[0.89,1.00]$ \\ $0.95[0.83,0.99]$ \\ $0.97[0.93,0.99]$ \\ $0.99[0.98,1.00]$ \\ $0.97[0.94,0.98]$ \\ $0.94[0.81,0.99]$ \\ $0.98[0.96,0.99]$ \\ $0.92[0.84,0.97]$ \\ $0.97[0.96,0.98]$ \\ $0.99[0.98,1.00]$ \\ $0.99[0.95,1.00]$ \\ $0.98[0.97,0.99]$ \\ $0.98[0.96,0.99]$ \\ $0.99[0.98,1.00]$ \\ $1.00[0.97,1.00]$ \\ $0.99[0.98,1.00]$ \\ $0.99[0.97,1.00]$ \\ $0.95[0.92,0.97]$ \\ $1.00[0.98,1.00]$
}

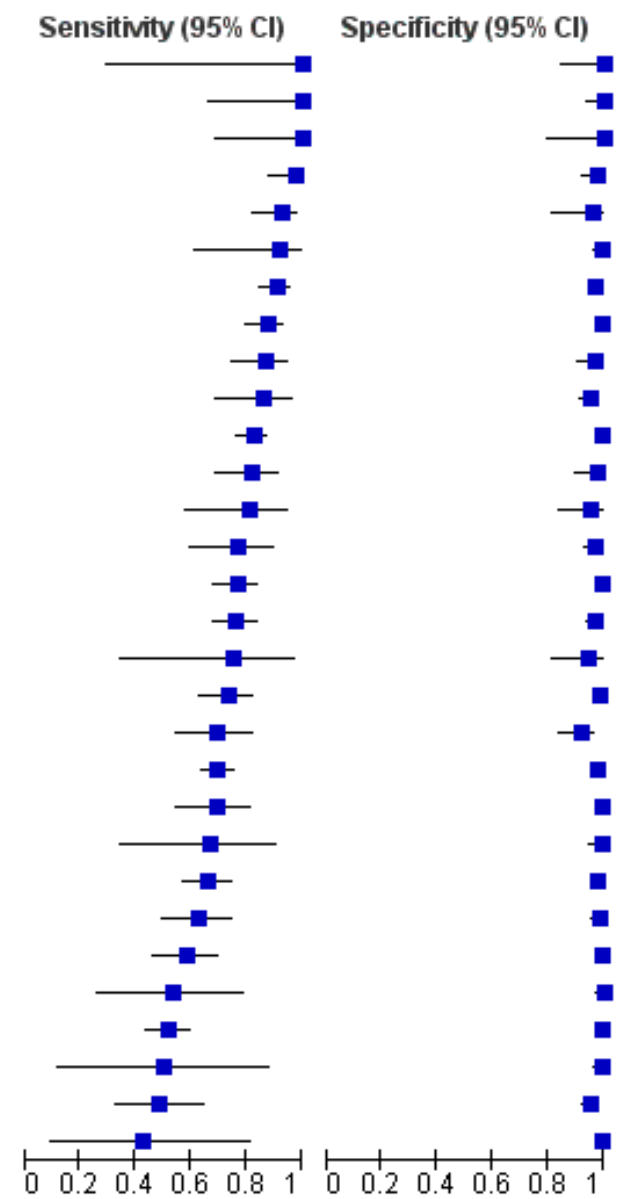

Scott 2011; Theron 2011). In HIV-negative participants, Xpert MTB/ RIF pooled sensitivity was $88 \%$ ( $83 \%$ to $92 \%$ ), higher than the sensitivity of $81 \%$ ( $75 \%$ to $86 \%$ ) in HIV-positive participants, although the 95\% Crls overlapped. In HIV-negative participants, Xpert MTB/RIF pooled specificity was $98 \%$ ( $97 \%$ to $99 \%$ ), the same as the pooled specificity of $98 \%$ (97\% to $99 \%$ ) in HIV-positive participants (Table 2; Figure 18). 
Figure 18. Forest plots of Xpert MTB/RIF sensitivity and specificity for detection of pulmonary tuberculosis, HIVnegative and HIV-positive participants compared within the same study. The squares represent the sensitivity and specificity of one study, the black line its confidence interval. FN: false-negative; FP: false-positive; TN: truenegative; TP: true-positive.

HIV-negative, within study comparisons

$\begin{array}{lrrrrrr}\text { Study } & \text { TP } & \text { FP } & \text { FN } & \text { TN } & \text { Sensitivity (95\% Cl) } & \text { Specificity (95\% Cl) } \\ \text { Bates 2013a } & 3 & 0 & 0 & 19 & 1.00[0.29,1.00] & 1.00[0.82,1.00] \\ \text { Boehme 2010 } & 335 & 1 & 26 & 173 & 0.93[0.90,0.95] & 0.99[0.97,1.00] \\ \text { Boehme 2011 } & 304 & 5 & 31 & 748 & 0.91[0.87,0.94] & 0.99[0.98,1.00] \\ \text { Boum 2016 } & 72 & 7 & 5 & 190 & 0.94[0.85,0.98] & 0.96[0.93,0.99] \\ \text { Calligaro 2015 } & 5 & 4 & 0 & 39 & 1.00[0.48,1.00] & 0.91[0.78,0.97] \\ \text { Calligaro 2017 } & 10 & 2 & 3 & 138 & 0.77[0.46,0.95] & 0.99[0.95,1.00] \\ \text { Dorman 2018 } & 143 & 9 & 16 & 315 & 0.90[0.84,0.94] & 0.97[0.95,0.99] \\ \text { Hanrahan 2013 } & 5 & 0 & 4 & 182 & 0.56[0.21,0.86] & 1.00[0.98,1.00] \\ \text { Hanrahan 2014 } & 120 & 13 & 29 & 689 & 0.81[0.73,0.87] & 0.98[0.97,0.99] \\ \text { Luetkemeyer 2016 } & 111 & 4 & 17 & 396 & 0.87[0.80,0.92] & 0.99[0.97,1.00] \\ \text { Pinyopornpanish 2015 } & 67 & 3 & 3 & 145 & 0.96[0.88,0.99] & 0.98[0.94,1.00] \\ \text { Rachow 2011 } & 28 & 2 & 2 & 41 & 0.93[0.78,0.99] & 0.95[0.84,0.99] \\ \text { Scott 2011 } & 17 & 0 & 2 & 53 & 0.89[0.67,0.99] & 1.00[0.93,1.00] \\ \text { Theron 2011 } & 15 & 1 & 2 & 127 & 0.88[0.64,0.99] & 0.99[0.96,1.00]\end{array}$

HIV-positive, within study comparisons
Figure 19 displays the summary ROC plot comparing Xpert MTB/RIF accuracy in HIV-negative and HIV-positive people in studies that in-

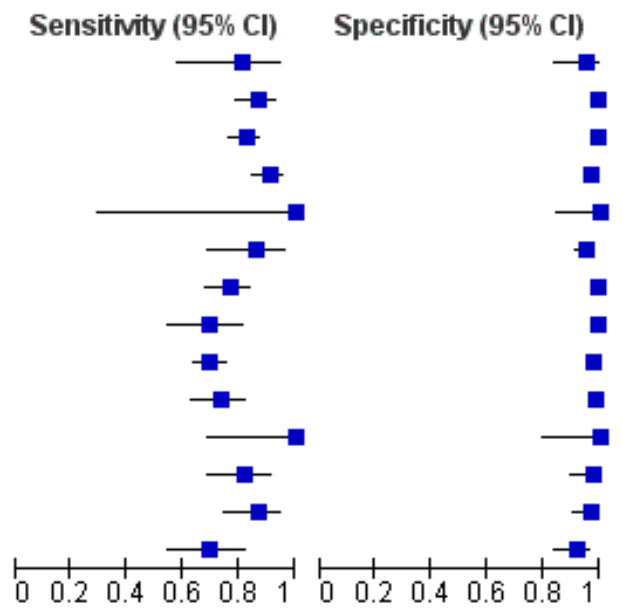

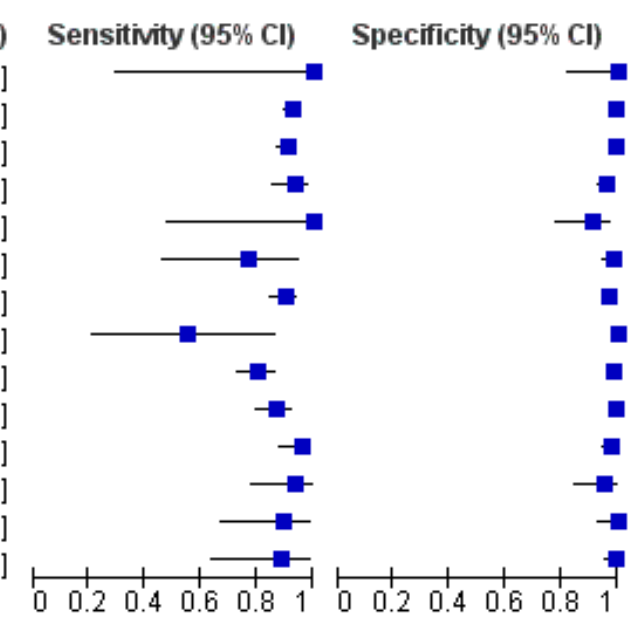

Specificity $(95 \% \mathrm{Cl})$ $0.95[0.83,0.99]$ $0.99[0.97,1.00]$ $0.99[0.98,1.00]$ $0.97[0.95,0.98]$ $1.00[0.85,1.00]$ $0.95[0.91,0.97]$ $0.99[0.98,1.00]$ $0.99[0.98,1.00]$ $0.97[0.96,0.98]$ $0.98[0.96,0.99]$ $1.00[0.79,1.00]$ $0.98[0.89,1.00]$ $0.97[0.90,0.99]$ $0.92[0.84,0.97$ volved both subgroups. The test demonstrated higher accuracy in HIV-negative people. 
Figure 19. Summary plots of Xpert MTB/RIF sensitivity and specificity for detection of pulmonary tuberculosis in HIV-negative people (red) and HIV-positive people (black). Each individual study is represented by an empty square. The of the square is proportional to the sample size of the study such that larger studies are represented by larger squares. The filled circle is the pooled median estimate for sensitivity and specificity. The solid curve represents the $95 \%$ credible region around the summary estimate; the dashed curves represent the $95 \%$ prediction region.

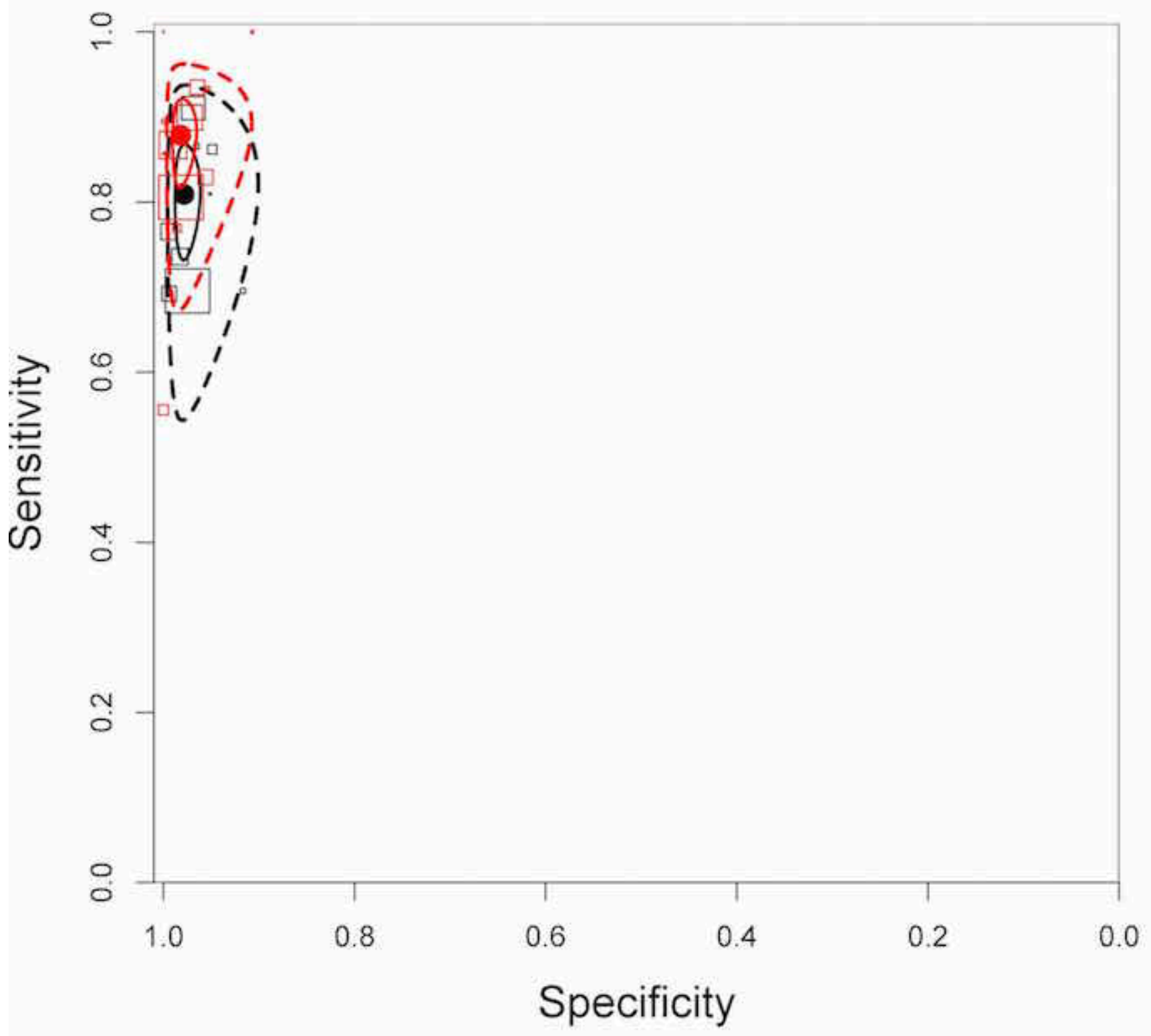

B.2.d. Xpert MTB/RIF versus Xpert Ultra for detection of PTB by HIV status, direct comparison

One study compared Xpert Ultra and Xpert MTB/RIF for detection of PTB by HIV status against a reference standard of multiple cultures (Dorman 2018). In HIV-negative participants, Xpert Ultra sensitivity
$(95 \% \mathrm{Cl})$ was $91 \%$ (86\% to $95 \%)$ compared to Xpert MTB/RIF sensitivity of $90 \%$ ( $84 \%$ to $94 \%$ ). In HIV-positive participants, Xpert Ultra yielded a higher sensitivity at $90 \%$ (82\% to $94 \%$ ), compared to Xpert MTB/RIF sensitivity of $77 \%$ ( $68 \%$ to $84 \%$ ), and a lower specificity at $96 \%(93 \%$ to $98 \%)$ compared to Xpert MTB/RIF specificity of $99 \%$ (98\% to $100 \%$ ) (Figure 20$)$. 
Figure 20. Forest plots comparing Xpert MTB/RIF and Xpert Ultra sensitivity and specificity for detection of pulmonary tuberculosis in HIV-negative and HIV-positive participants. The individual studies are ordered by decreasing sensitivity. The squares represent the sensitivity and specificity of one study, the black line its $\mathrm{Cl}$. TP= true positive; $\mathrm{FP}$ = false positive; $\mathrm{FN}$ = false negative; $\mathrm{TN}=$ true negative.

HIV-negative, Xpert MTB/RIF, direct comparison Xpert MTB/RIF vs Xpert Ultra

\begin{tabular}{|c|c|c|c|c|c|c|c|c|}
\hline Stucly & TP & FP & FN & TN & Sensitivity (95\% Cl) & Specificity (95\% Cl) & Sensitivity (95\% Cl) & Specificity (95\% Cl) \\
\hline Dorman 2018 & 143 & 9 & 16 & 315 & $0.90[0.84,0.94]$ & $0.97[0.95,0.99]$ & & \\
\hline
\end{tabular}

HIV-negative, Xpert Ultra, direct comparison Xpert MTB/RIF vs Xpert Ultra
Stucly
TP FP FN
TN Set
ensitivity $(95 \% \mathrm{Cl})$
Specificity $(95 \% \mathrm{Cl})$
Sensitivity $(95 \% \mathrm{Cl})$
Specificity (95\% Cl)
Dorman 2018
$\begin{array}{lllll}145 & 17 & 14 & 307\end{array}$
$0.91[0.86,0.95]$
$0.95[0.92,0.97]$
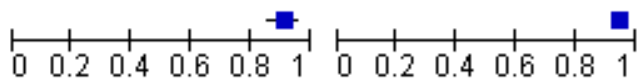

HIV-positive, Xpert MTB/RIF, direct comparison Xpert MTB/RIF vs Xpert Ultra

$\begin{array}{lrrrrrr}\text { Study } & \text { TP } & \text { FP } & \text { FN } & \text { TN } & \text { Sensitivity }(95 \% \text { Cl) } & \text { Specificity }(95 \% \text { Cl) } \\ \text { Dorman } 2018 & 88 & 2 & 27 & 315 & 0.77[0.68,0.84] & 0.99[0.98,1.00]\end{array}$

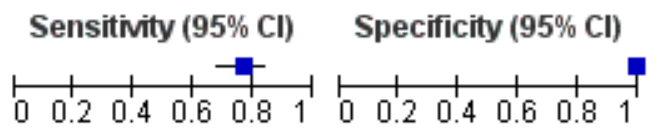

HIV-positive, Xpert Ultra, direct comparison Xpert MTB/RIF vs Xpert Ultra

\begin{tabular}{|c|c|c|c|c|c|c|c|c|}
\hline Study & TP & FP & FN & TN & Sensitivity (95\% Cl) & Specificity (95\% Cl) & Sensitivity (95\% Cl) & Specificity $(95 \% \mathrm{Cl})$ \\
\hline Dorman 2018 & 103 & 14 & 12 & 303 & $0.90[0.82,0.94]$ & $0.96[0.93,0.98]$ & & \\
\hline
\end{tabular}

B.3. Xpert MTB/RIF accuracy for detection of PTB in participants with a history of tuberculosis or previous tuberculosis treatment

\section{B.3.a. Xpert MTB/RIF accuracy in participants with a history of} tuberculosis

Eleven studies (4196 participants) reported a higher percentage (> $25 \%$ ) of participants with a history of tuberculosis (Adelman 2015; Al-Darraji 2013; Boehme 2010; Kawkitinarong 2017; Ko 2016; Lawn 2011; Mutingwende 2015; O'Donnell 2015; Reddy 2017; Reechaipichitkul 2017; Theron 2011) and 16 studies (8205 participants) reported a lower percentage $(\leq 25 \%)$ of participants with a history of tuberculosis (Balcha 2014; Barmankulova 2015; Bates 2013a; Bjerrum 2016; Boehme 2010; Boum 2016; Carriquiry 2012; Dorman 2018; Hanrahan 2013; Helb 2010; LaCourse 2016; Luetkemeyer 2016; Mbelele 2017; Scott 2017; Sohn 2014; Yoon 2017). In studies with a higher percentage of participants with previous tuberculosis, Xpert MTB/RIF pooled sensitivity (95\% CrI) was $86 \%$ (82\% to $89 \%$ ), similar to the pooled sensitivity of $85 \%$ ( $81 \%$ to $89 \%$ ) in studies with a lower percentage of participants with previous tuberculosis. In studies with a higher percentage of participants with previous tuberculosis, Xpert MTB/RIF pooled specificity was 97\% (95\% to $98 \%$ ), lower than the specificity of $99 \%$ (98\% to $99 \%$ ) in studies with a lower percentage of participants with previous tuberculosis (Table 2).

\section{B.3.b. Xpert MTB/RIF accuracy in participants who had received previous tuberculosis treatment}

We identified three studies involving 999 participants that preferentially enrolled participants who had received previous tuberculosis treatment (Meawed 2016; Metcalfe 2015; Pimkina 2015). Sensitivity estimates ranged from $92 \%$ to $98 \%$ and specificity estimates from $75 \%$ to $92 \%$. Xpert MTB/RIF pooled sensitivity and specificity (95\% Crl) were $94 \%$ (87\% to $97 \%$ ) and $89 \%$ (75\% to $95 \%)$ respectively. Xpert MTB/RIF pooled specificity was considerably lower than the pooled specificity of $98 \%$ (97\% to $98 \%)$ in the primary analysis (70 studies).

\section{B.4. Xpert MTB/RIF accuracy by tuberculosis burden}

There were 39 studies (21,965 participants) conducted in high tuberculosis burden countries and 33 studies ( 5272 participants) conducted in countries not considered to be high tuberculosis burden. In countries with high tuberculosis burden, Xpert MTB/RIF pooled sensitivity $(95 \% \mathrm{Crl})$ was $86 \%(82 \%$ to $89 \%)$, similar to the pooled sensitivity of $85 \%$ ( $81 \%$ to $89 \%$ ) in countries not considered to be high tuberculosis burden. In countries with high tuberculosis burden, Xpert MTB/RIF pooled specificity was 97\% (95\% to 98\%), lower than the pooled specificity of $99 \%$ (98\% to $99 \%)$ in countries not considered to be high tuberculosis burden (Table 2).

\section{B.5. Xpert MTB/RIF accuracy by TB/HIV burden}

There were 42 studies (24,412 participants) conducted in high TB/ HIV burden countries and 30 studies (12,825 participants) conducted in countries not considered to be high TB/HIV burden. In countries with high TB/HIV burden, Xpert MTB/RIF pooled sensitivity $(95 \% \mathrm{Crl})$ was $83 \%(80 \%$ to $87 \%)$, lower than the pooled sensitivity of $88 \%$ (84\% to $90 \%$ ) in countries not considered to be high TB/HIV burden, although there was considerable overlap in the Crls around these estimates. In countries with high TB/HIV burden, Xpert MTB/ RIF pooled specificity was $97 \%$ (95\% to $98 \%$ ), lower than the pooled specificity of $99 \%$ (98\% to $99 \%$ ) in countries not considered to be high TB/HIV burden (Table 2). 


\section{B.6. Xpert MTB/RIF accuracy by setting that ran the test}

There were 10 studies (5816 participants) that ran Xpert MTB/RIF at point of care or in a peripheral setting (Al-Darraji 2013; Calligaro 2017; Chaisson 2014; Chew 2016; Geleta 2015; Hanrahan 2013; Huang 2015; Kurbaniyazova 2017; Shao 2017; Theron 2014a), and 60 studies (31,421 participants) that ran Xpert MTB/RIF in an intermediate or central-level laboratory. In studies running Xpert MTB/ RIF at point of care or in a peripheral setting, the pooled sensitivity $(95 \% \mathrm{Crl})$ was $83 \%$ ( $75 \%$ to $89 \%)$, lower than the sensitivity of $85 \%$ $(83 \%$ to $88 \%$ ) in studies running Xpert MTB/RIF in an intermediate or central-level laboratory. In peripheral settings, the pooled specificity was $97 \%$ (94\% to $99 \%$ ), lower than the pooled specificity of $98 \%(97 \%$ to $98 \%)$ in more advanced laboratories. However, there was considerable overlap in Crls around these accuracy estimates (Table 2).

\section{B.7. Xpert MTB/RIF accuracy by tuberculosis prevalence}

The prevalence of PTB cases confirmed by culture in the studies ranged from $0.8 \%$ (Chen 2017) to $100 \%$ (Friedrich 2011). Based on a median tuberculosis prevalence of $26 \%$, in settings with tuberculosis prevalence above $26 \%$, Xpert MTB/RIF pooled sensitivity (95\% $\mathrm{Crl}$ ) was $89 \%$ ( $87 \%$ to $91 \%$ ), higher than the pooled sensitivity of $79 \%$ ( $75 \%$ to $83 \%$ ) in settings with tuberculosis prevalence at or below $26 \%$. The corresponding pooled specificities were $96 \%$ (94\% to $97 \%$ ) and $99 \%$ (98\% to $99 \%$ ) (Table 2 ).

\section{Uninterpretable results, detection of PTB}

Among 47 studies involving 31,979 tests, the pooled proportion of uninterpretable test results for Xpert MTB/RIF was very low, at $1.1 \%$ $(0.7 \%$ to $1.5 \%)$. In the study comparing Xpert Ultra and Xpert MTB/ RIF, of 2001 specimens initially tested, uninterpretable results were found for 79 specimens (4\%) with Xpert Ultra and 39 specimens (2\%) with Xpert MTB/RIF. After exclusion of errors related to instrumentation, uninterpretable results were found for 64 specimens (3\%) with Xpert Ultra and 28 specimens (1\%) with Xpert MTB/RIF (Dorman 2018).

\section{Detection of rifampicin resistance}

\section{A. Xpert MTB/RIF for detection of rifampicin resistance}

\section{1.a. Primary analysis, Xpert MTB/RIF}

The 57 studies involved 8287 specimens, of which 1775 were rifampicin-resistant, median 88 specimens (range 1 to 250 ). Six studies accounted for most $(63 \%, 1127 / 1775)$ of the rifampicin-resistant specimens (Boehme 2010; Boehme 2011; Dorman 2018; Huang 2015; Kurbaniyazova 2017; Sharma 2015) (Figure 21). Although there was heterogeneity in sensitivity estimates (ranging from $75 \%$ to $100 \%$ ), in general there was less variability among studies with a higher number of rifampicin-resistant specimens. Specificity showed less variability than sensitivity, ranging from $83 \%$ to $100 \%$. 
Figure 21. Forest plots of Xpert MTB/RIF sensitivity and specificity for detection of rifampicin resistance. The individual studies are ordered by decreasing sensitivity. The squares represent the sensitivity and specificity of one study, the black line its $\mathrm{Cl}$. TP = true positive; $\mathrm{FP}=$ false positive; $\mathrm{FN}=$ false negative; $\mathrm{TN}$ = true negative .

\begin{tabular}{|c|c|c|c|c|c|c|c|c|}
\hline Stuchy & TP & FP & FN & TN & Sensitivity (95\% Cl) & Specificity (95\% Cl) & Sensitivity (95\% Cl) & Specificity (95\% Cl) \\
\hline Metcalfe 2016 & 54 & 2 & 0 & 90 & $1.00[0.93,1.00]$ & $0.98[0.92,1.00]$ & & $\rightarrow$ \\
\hline Ali 2017 & 46 & 0 & 0 & 80 & $1.00[0.92,1.00]$ & $1.00[0.95,1.00]$ & $\rightarrow$ & \\
\hline Pimkina 2015 & 39 & 4 & 0 & 221 & $1.00[0.91,1.00]$ & $0.98[0.96,1.00]$ & $\rightarrow$ & \\
\hline Meawed 2016 & 37 & 0 & 0 & 16 & $1.00[0.91,1.00]$ & $1.00[0.79,1.00]$ & $\rightarrow$ & $\longrightarrow$ \\
\hline Makamure 2017 & 25 & 5 & 0 & 34 & $1.00[0.86,1.00]$ & $0.87[0.73,0.96]$ & $\rightarrow$ & $\longrightarrow$ \\
\hline N'Guessan 2016 & 23 & 1 & 0 & 39 & $1.00[0.85,1.00]$ & $0.97[0.87,1.00]$ & $\rightarrow$ & $\rightarrow$ \\
\hline Tsuyuguchi 2017 & 22 & 3 & 0 & 176 & $1.00[0.85,1.00]$ & $0.98[0.95,1.00]$ & $\longrightarrow$ & - \\
\hline Nosova $2013 a$ & 13 & 0 & 0 & 33 & $1.00[0.75,1.00]$ & $1.00[0.89,1.00]$ & $\longrightarrow$ & $\rightarrow$ \\
\hline Mokaddas 2015 & 7 & 1 & 0 & 279 & $1.00[0.59,1.00]$ & $1.00[0.98,1.00]$ & & घ \\
\hline Carriquiry 2012 & 6 & 3 & 0 & 30 & $1.00[0.54,1.00]$ & $0.91[0.76,0.98]$ & & \\
\hline Luetkemeyer 2016 & 5 & 1 & 0 & 154 & $1.00[0.48,1.00]$ & $0.99[0.96,1.00]$ & & ㅂ \\
\hline Theron 2011 & 5 & 1 & 0 & 151 & $1.00[0.48,1.00]$ & $0.99[0.96,1.00]$ & $\longrightarrow$ & घ \\
\hline Kim CH 2015 & 4 & 1 & 0 & 31 & $1.00[0.40,1.00]$ & $0.97[0.84,1.00]$ & & $\rightarrow$ \\
\hline Lawn 2011 & 4 & 3 & 0 & 48 & $1.00[0.40,1.00]$ & $0.94[0.84,0.99]$ & & $\rightarrow$ \\
\hline Ssengooba 2014 & 4 & 0 & 0 & 90 & $1.00[0.40,1.00]$ & $1.00[0.96,1.00]$ & & \\
\hline Tang 2017 & 4 & 1 & 0 & 63 & $1.00[0.40,1.00]$ & $0.98[0.92,1.00]$ & & - \\
\hline Friedrich 2011 & 3 & 0 & 0 & 90 & $1.00[0.29,1.00]$ & $1.00[0.96,1.00]$ & & \\
\hline Sah 2017 & 3 & 0 & 0 & 29 & $1.00[0.29,1.00]$ & $1.00[0.88,1.00]$ & & \\
\hline Balcells 2012 & 2 & 0 & 0 & 10 & $1.00[0.16,1.00]$ & $1.00[0.69,1.00]$ & & \\
\hline Chikaonda 2017 & 2 & 1 & 0 & 185 & $1.00[0.16,1.00]$ & $0.99[0.97,1.00]$ & & \\
\hline Lee 2013 & 2 & 0 & 0 & 29 & $1.00[0.16,1.00]$ & $1.00[0.88,1.00]$ & & $\rightarrow$ \\
\hline Park 2013 & 2 & 1 & 0 & 16 & $1.00[0.16,1.00]$ & $0.94[0.71,1.00]$ & & \\
\hline Rice 2017 & 2 & 2 & 0 & 116 & $1.00[0.16,1.00]$ & $0.98[0.94,1.00]$ & & $\rightarrow$ \\
\hline Williamson 2012 & 2 & 1 & 0 & 64 & $1.00[0.16,1.00]$ & $0.98[0.92,1.00]$ & & $\rightarrow$ \\
\hline Calligaro 2015 & 1 & 0 & 0 & 10 & $1.00[0.03,1.00]$ & $1.00[0.69,1.00]$ & & \\
\hline Le Palud 2014 & 1 & 0 & 0 & 15 & $1.00[0.03,1.00]$ & $1.00[0.78,1.00]$ & & \\
\hline Lippincott 2014 & 1 & 0 & 0 & 5 & $1.00[0.03,1.00]$ & $1.00[0.48,1.00]$ & & $\rightarrow$ \\
\hline Sohn 2014 & 1 & 1 & 0 & 9 & $1.00[0.03,1.00]$ & $0.90[0.55,1.00]$ & & $=$ \\
\hline Zeka 2011 & 1 & 0 & 0 & 34 & $1.00[0.03,1.00]$ & $1.00[0.90,1.00]$ & & $\rightarrow$ \\
\hline Theron 2013 & 1 & 0 & 0 & 24 & $1.00[0.03,1.00]$ & $1.00[0.86,1.00]$ & & $\longrightarrow$ \\
\hline Kurbatova 2013 & 55 & 2 & 1 & 42 & $0.98[0.90,1.00]$ & $0.95[0.85,0.99]$ & $\rightarrow$ & $\rightarrow$ \\
\hline Boehme 2010 & 200 & 10 & 5 & 505 & $0.98[0.94,0.99]$ & $0.98[0.96,0.99]$ & $=$ & घ \\
\hline Barmankulova 2015 & 91 & 8 & 3 & 89 & $0.97[0.91,0.99]$ & $0.92[0.84,0.96]$ & $\rightarrow$ & $\rightarrow$ \\
\hline Huang 2015 & 128 & 1 & 6 & 20 & $0.96[0.91,0.98]$ & $0.95[0.76,1.00]$ & $\rightarrow$ & $\rightarrow$ \\
\hline Dorman 2018 & 167 & 7 & 8 & 369 & $0.95[0.91,0.98]$ & $0.98[0.96,0.99]$ & 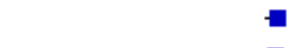 & घ \\
\hline Sharma 2015 & 104 & 7 & 6 & 305 & $0.95[0.89,0.98]$ & $0.98[0.95,0.99]$ & $\Rightarrow$ & - \\
\hline Boehme 2011 & 236 & 14 & 14 & 796 & $0.94[0.91,0.97]$ & $0.98[0.97,0.99]$ & 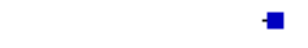 & घ \\
\hline Liu 2017 & 15 & 0 & 1 & 383 & $0.94[0.70,1.00]$ & $1.00[0.99,1.00]$ & $\longrightarrow$ & $\boldsymbol{\square}$ \\
\hline Zetola 2014 & 51 & 1 & 4 & 314 & $0.93[0.82,0.98]$ & $1.00[0.98,1.00]$ & $\rightarrow$ & - \\
\hline Kurbaniyazova 2017 & 228 & 49 & 25 & 476 & $0.90[0.86,0.94]$ & $0.91[0.88,0.93]$ & $=$ & $=$ \\
\hline Lorent 2015 & 24 & 6 & 3 & 69 & $0.89[0.71,0.98]$ & $0.92[0.83,0.97]$ & $\rightarrow-$ & $\rightarrow$ \\
\hline Kwak 2013 & 8 & 0 & 1 & 90 & $0.89[0.52,1.00]$ & $1.00[0.96,1.00]$ & $\rightarrow-$ & 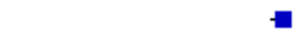 \\
\hline Singh 2016 & 14 & 0 & 2 & 56 & $0.88[0.62,0.98]$ & $1.00[0.94,1.00]$ & - & $\rightarrow$ \\
\hline Huh 2014 & 6 & 1 & 1 & 90 & $0.86[0.42,1.00]$ & $0.99[0.94,1.00]$ & $\rightarrow-$ & $\rightarrow$ \\
\hline O'Donnell 2015 & 20 & 4 & 4 & 84 & $0.83[0.63,0.95]$ & $0.95[0.89,0.99]$ & $\longrightarrow-$ & $\rightarrow$ \\
\hline Scott 2011 & 4 & 2 & 1 & 10 & $0.80[0.28,0.99]$ & $0.83[0.52,0.98]$ & & $=-$ \\
\hline Kawkitinarong 2017 & 12 & 2 & 4 & 210 & $0.75[0.48,0.93]$ & $0.99[0.97,1.00]$ & & \\
\hline Al-Darraji 2013 & 0 & 0 & 0 & 8 & Not estimable & $1.00[0.63,1.00]$ & & \\
\hline Bates 2013a & 0 & 0 & 1 & 20 & $0.00[0.00,0.97]$ & $1.00[0.83,1.00]$ & & $\longrightarrow$ \\
\hline Barnard 2015 & 0 & 0 & 0 & 36 & Not estimable & $1.00[0.90,1.00]$ & & $\rightarrow$ \\
\hline Hanif 2011 & 0 & 0 & 0 & 54 & Not estimable & $1.00[0.93,1.00]$ & & $\rightarrow$ \\
\hline Zmak 2013 & 0 & 0 & 0 & 6 & Not estimable & $1.00[0.54,1.00]$ & & \\
\hline Rachow 2011 & 0 & 0 & 0 & 59 & Not estimable & $1.00[0.94,1.00]$ & & $\rightarrow$ \\
\hline Moussa 2016 & 0 & 0 & 0 & 67 & Not estimable & $1.00[0.95,1.00]$ & & $\rightarrow$ \\
\hline Safianowska 2012 & 0 & 0 & 0 & 15 & Not estimable & $1.00[0.78,1.00]$ & & 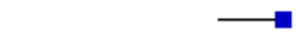 \\
\hline
\end{tabular}


Xpert MTB/RIF pooled sensitivity and specificity (95\% Crl) were $96 \%$ ( $94 \%$ to $97 \%$ ) and $98 \%$ (98\% to $99 \%$ ) (48 studies, 8020 participants) (Table 1).

\section{1.b. Primary analysis, Xpert Ultra}

One study (Dorman 2018) evaluated Xpert Ultra and Xpert MTB/RIF in the same participants for detection of rifampicin resistance. The sensitivity and specificity estimates were similar. Xpert Ultra sensitivity and specificity $(95 \% \mathrm{Cl})$ were $95 \%(90 \%$ to $98 \%)$ and $98 \%(97 \%$ to $99 \%$ ) respectively (551 specimens, including 175 rifampicin-resistant specimens); while Xpert MTB/RIF sensitivity and specificity were $95 \%$ (91\% to $98 \%)$ and $98 \%$ (96\% to $99 \%)$ respectively (552 specimens, including 175 rifampicin-resistant specimens).

\section{B. Investigations of heterogeneity, rifampicin resistance}

B.1. Xpert MTB/RIF accuracy for detection of rifampicin resistance by MDR-TB burden

In settings with high MDR-TB burden, Xpert MTB/RIF pooled sensitivity ( $95 \% \mathrm{Crl}$ ) was $95 \%$ (93\% to $97 \%$ ), lower than the pooled sensitivity of $97 \%$ (93\% to $99 \%$ ) for studies not in the high MDR-TB category. The corresponding pooled specificities (95\% Crl) were $98 \%$ (96\% to $99 \%$ ) and $99 \%$ (95\% Crl 98\% to 99\%) (Table 3). For both sensitivity and specificity, the $95 \%$ Crls in the two groups overlapped, suggesting that MDR-TB burden did not have an effect on the accuracy estimates.

\section{B.2. Xpert MTB/RIF accuracy for detection of rifampicin resistance by} previous tuberculosis treatment

Several studies designed to enrol participants suspected of MDRTB had high percentages of participants previously treated for tuberculosis (Lorent 2015; Makamure 2017; Meawed 2016; Metcalfe 2016; N'Guessan 2016; Pimkina 2015; Zetola 2014). In these studies (7 studies, 1062 participants), Xpert MTB/RIF pooled sensitivity at $98 \%$ (95\% Crl 94\% to $99 \%$ ) was higher than the pooled sensitivity of 95\% (93\% to 97\%) in studies that did not preferentially enrol previously treated participants (41 studies, 6958 participants); and conversely, pooled specificity was lower at $97 \%$ (93\% to $99 \%$ ) than the pooled specificity of $99 \%$ (95\% Crl $98 \%$ to $99 \%$ ) in studies that did not preferentially enrol previously treated participants. However, for both sensitivity and specificity estimates the Crls overlapped, suggesting that previous tuberculosis treatment did not have an effect on Xpert MTB/RIF accuracy for detection of rifampicin resistance (Table 3).

\section{B.3. Xpert MTB/RIF accuracy for detection of rifampicin resistance by prevalence of rifampicin resistance}

Based on a median prevalence of rifampicin resistance of $11 \%$, in studies with prevalence of rifampicin resistance above $11 \%$, Xpert MTB/RIF pooled sensitivity ( $95 \% \mathrm{Crl}$ ) was $96 \%$ (94\% to $97 \%$ ), higher than the pooled sensitivity of $94 \%(95 \% \mathrm{Crl} 89 \%$ to $97 \%$ ) for studies with prevalence of rifampicin resistance at or below $11 \%$, although the Crls overlapped. The corresponding pooled specificities were $97 \%$ (96\% to $98 \%$ ) and $99 \%$ (99\% to $100 \%$ ) (Table 3).

\section{Indeterminate results, rifampicin resistance}

Among 21 studies involving 3591 tests, the pooled proportion of Xpert MTB/RIF indeterminate test results was very low, at $0.9 \%$ $(0.4 \%$ to $1.5 \%)$. In the study comparing Xpert Ultra and Xpert MTB/ RIF, of 684 specimens tested, indeterminate results were found for
16 specimens (2\%) with Xpert Ultra and four specimens (1\%) with Xpert MTB/RIF (Dorman 2018).

\section{Sensitivity analyses}

For Xpert MTB/RIF for detection of PTB, we undertook sensitivity analyses by limiting inclusion in the meta-analysis to:

- Studies that explicitly represented the use of the index test for the diagnosis of individuals thought to have tuberculosis. We excluded studies that involved HIV-positive participants irrespective of tuberculosis symptoms;

- Studies where a single specimen yielded a single Xpert MTB/RIF result for a given participant. We excluded studies that included more specimens than participants;

- Studies that only included untreated participants;

- Studies that used liquid culture as the reference standard;

- Studies where a consecutive or random sample of participants were enrolled;

- Studies where the reference standard was blinded;

- Studies that only used fresh specimens;

- Studies that accounted for all participants in the analysis. We excluded studies where we answered 'no' or 'unclear' to the QUADAS-2 Flow and Timing signalling question: Were all patients included in the analysis?;

- Studies with exclusion of two large multicentre studies (Boehme 2010; Boehme 2011).

These sensitivity analyses made little difference to any of the findings (Table 4).

\section{Other analyses}

\section{NTM}

Twenty-eight studies evaluating Xpert MTB/RIF and involving 8901 participants provided data on a variety of NTMs that grew from the specimens tested, to look for evidence of cross-reactivity: one NTM (Al-Darraji 2013); four NTMs (Balcells 2012); two NTMs (Barnard 2015); 50 NTMs (Bjerrum 2016); one NTM (Chaisson 2014); 16 NTMs (Cowan 2017); three NTMs (Davis 2014); 12 NTMs (Kim CH 2015); one NTM (Kurbatova 2013); nine NTMs (Le Palud 2014); 16 NTMs (Lee 2013); 40 NTMs (Lippincott 2014); 14 NTMs (Lorent 2015); 95 NTMs (Luetkemeyer 2016); 20 NTMs (Moure 2011); four NTMs (Nosova 2013a); 10 NTMs (Pinyopornpanish 2015); 45 NTMs (Rachow 2011); 122 NTMs (Rice 2017); seven NTMs (Safianowska 2012); five NTMs (Scott 2011); three NTMs (Sohn 2014); 19 NTMs (Ssengooba 2014); two NTMs (Tang 2017); eight NTMs (Theron 2011); three NTMs (Van Rie 2013); 22 NTMs (Williamson 2012); and two NTMs (Zmak 2013). Among these 28 studies comprising 536 NTMs, Xpert MTB/RIF was positive in 16 specimens that grew NTMs, pooled proportion $2.0 \%$ $(0.4 \%$ to $4.4 \%)$. NTM data for Xpert Ultra were not reported.

\section{DISCUSSION}

This updated Cochrane Review on the diagnostic accuracy of Xpert MTB/RIF (Xpert MTB/RIF) and Xpert MTB/RIF Ultra (Xpert Ultra) for detection of tuberculosis and rifampicin resistance in adults summarizes the current literature and integrates 77 new studies ( $81 \%$ of the total 95 included studies), identified since the previous Cochrane Review (Steingart 2014). The findings in this update are consistent with those reported previously. 


\section{Summary of main results}

- For detection of PTB, Xpert MTB/RIF sensitivity and specificity were $85 \%$ and $98 \%$.

- Xpert MTB/RIF sensitivity was $98 \%$ for smear-positive, culture-positive tuberculosis, and $67 \%$ for smear-negative, culture-positive tuberculosis.

- Xpert MTB/RIF sensitivity for PTB was $88 \%$ in HIV-negative people and $81 \%$ in HIV-positive people.

- For detection of PTB, the pooled proportion of Xpert MTB/RIF uninterpretable test results was very low.

- For detection of rifampicin resistance, Xpert MTB/RIF sensitivity and specificity were $96 \%$ and $98 \%$.

- For detection of rifampicin resistance, the pooled proportion of Xpert MTB/RIF indeterminate test results was very low.

- In the one study that directly compared Xpert Ultra and Xpert MTB/RIF, Xpert Ultra yielded a higher sensitivity (88\%) than Xpert MTB/RIF (83\%), and a lower specificity (96\%) than Xpert MTB/RIF (98\%)

- In the one study that directly compared Xpert Ultra and Xpert MTB/RIF, for detection of smear-negative culture-positive tuberculosis, Xpert Ultra yielded a higher sensitivity (63\%) than Xpert MTB/RIF (46\%), and a lower specificity (96\%) than Xpert MTB/ $\operatorname{RIF}(98 \%)$.

- In the one study that directly compared Xpert Ultra and Xpert MTB/RIF, for detection of PTB in HIV-positive people, Xpert Ultra yielded a higher sensitivity (90\%) than Xpert MTB/RIF (77\%), and a lower specificity (96\%) than Xpert MTB/RIF (99\%).

\section{Xpert MTB/RIF for PTB}

Results of these studies indicate that, in theory, for a population of 1000 people where 100 have tuberculosis on culture, 103 would be Xpert MTB/RIF-positive and 18 (17\%) would not have tuberculosis (false-positives); 897 would be Xpert MTB/RIF-negative and 15 (2\%) would have tuberculosis (false-negatives) (Summary of findings 1 ).

\section{Xpert Ultra for PTB}

Results of these studies indicate that, in theory, for a population of 1000 people where 100 have tuberculosis on culture, 124 would be Xpert Ultra-positive; of these, $36(29 \%)$ would not have tuberculosis (false-positives); and 876 would be Xpert Ultra-negative; of these, $12(1 \%)$ would have tuberculosis (false-negatives) (Summary of findings 1 ).

\section{Xpert MTB/RIF for rifampicin resistance}

Results of these studies indicate that, in theory, for a population of 1000 people where 100 have rifampicin-resistant tuberculosis, 114 would be positive for rifampicin-resistant tuberculosis; of these 18 (16\%) would not have rifampicin resistance (false-positives); and 886 would be would be negative for rifampicin-resistant tuberculosis; of these, four $(0.4 \%)$ would have rifampicin resistance (falsenegatives) (Summary of findings 2 ).

\section{Xpert Ultra for rifampicin resistance}

Results of these studies indicate that, in theory, for a population of 1000 people where 100 have rifampicin-resistant tuberculosis, 113 would be positive for rifampicin-resistant tuberculosis; of these, $18(16 \%)$ would not have rifampicin resistance (false-positives); and 887 would be negative for rifampicin-resistant tuberculosis; of these, five (1\%) would have rifampicin resistance (false-negatives) (Summary of findings 2).

\section{Xpert MTB/RIF performance in different subgroups and settings}

Xpert MTB/RIF detects DNA sequences of $M$ tuberculosis after amplification and has a lower limit of detection of $131 \mathrm{CFUs} / \mathrm{mL}$ (Helb 2010). The cycle threshold value $\left(C_{T}\right)$ is the number of PCR cycles after which Xpert MTB/RIF probes successfully detect $M$ tuberculosis DNA in a given sample. Xpert MTB/RIF $C_{T}$ values are strongly correlated with AFB smear status (Lange 2017). The lower sensitivity of Xpert MTB/RIF in individuals with AFB smear-negative PTB is related to the lower bacillary burden and higher associated $C_{T}$ value compared to individuals with AFB smear-positive PTB. Individuals with PTB and HIV co-infection are more likely to have smear-negative tuberculosis, which implies a lower bacillary burden and higher mean $C_{T}$ values on Xpert testing (Beynon 2018; Lange 2017), and this is the likely mechanism for the lower sensitivity of Xpert MTB/ RIF for the diagnosis of tuberculosis in people living with HIV.

In individuals with a history of treatment for tuberculosis, we found that Xpert MTB/RIF pooled specificity (89\%) was lower than the pooled specificity in the primary analysis (98\%). This is consistent with findings from the literature that Xpert MTB/RIF may be positive at the end of tuberculosis treatment despite cure (Friedrich 2013; Theron 2016; Theron 2018), and may rarely remain positive for up to five years after tuberculosis treatment (Boyles 2014). Among individuals with a history of tuberculosis treatment, the included Xpert Ultra paper found that specificity improved as time since tuberculosis treatment increased, and approximated to that of participants without a history of tuberculosis treatment when elapsed time was seven years (Dorman 2018). Xpert MTB/RIF does not distinguish dead from living bacilli and it is not surprising at the end of treatment to have Xpert MTB/RIF-positive results (false-positives) and hence lower specificity. $C_{T}$ values may help in differentiating between true-positive and false-positive Xpert MTB/RIF results in people with a prior history of tuberculosis, with lower values in those with tuberculosis recurrence compared to those with falsepositive Xpert MTB/RIF (Theron 2016; Theron 2018).

In countries with high TB/HIV burden, we found that Xpert MTB/ RIF pooled specificity $(97 \%)$ was lower than the pooled specificity (99\%) in countries not considered to have a high TB/HIV burden. This difference in specificity may be due to other factors, such as the laboratory level of MTB/RIF testing rather than the presence of HIV infection, as specificity in HIV-positive and HIV-negative individuals was similar. Supporting the importance of laboratory setting, Xpert MTB/RIF specificity was lower at point of care and in peripheral laboratories compared to intermediate and central laboratories.

For prevalence of tuberculosis, in comparing settings with a higher or lower prevalence of tuberculosis, for both Xpert MTB/RIF sensitivity and specificity, we found that the $95 \%$ credible intervals (Crls) in the two groups did not overlap, suggesting an association of prevalence of tuberculosis with the accuracy estimates. In comparing settings with a higher or lower prevalence of rifampicin resistance, we found that the Crls for specificity did not overlap, suggesting an association of prevalence of rifampicin resistance with the specificity estimates. Changes in disease prevalence have often been found to be associated with other important changes, such as 
changes in the disease spectrum, which may affect diagnostic accuracy estimates (Leeflang 2013).

Sensitivity and specificity depend on the performance of a test in a particular situation, defined by the population, the setting, and prior testing. In a different population or setting or with a different testing strategy, the sensitivity and specificity are likely to change (Bossuyt 2008). However, our sensitivity analyses of different specimen numbers and conditions did not change Xpert MTB/ RIF performance. We did find that among specimens that were culture-positive for NTM, false-positive Xpert MTB/RIF results occurred in $2.0 \%(0.4 \%$ to $4.4 \%)$. Although there have been suggestions that certain nontuberculous mycobacterial species (e.g. M malmoense) may give false-positive Xpert MTB/RIF results due to weak cross hybridization (Agizew 2017), the false-positive rate in specimens culture-positive for NTM was similar to the overall frequency of false positives.

Our systematic review included only one study that evaluated Xpert Ultra (Dorman 2018). This multicentre study found that Xpert Ultra yielded higher sensitivity at $88 \%(95 \% \mathrm{Cl} 85 \%$ to $91 \%)$ compared to Xpert MTB/RIF sensitivity of $83 \%$ ( $79 \%$ to $86 \%$ ), but lower specificity of $96 \%$ (94\% to $97 \%$ ) compared to Xpert MTB/RIF specificity of $98 \%$ (97\% to 99\%) (Dorman 2018). This study performed several post hoc analyses that evaluated the impact of changing the classification of Xpert Ultra trace calls, which in the primary analysis were considered positive for the identification of $M$ tuberculosis. Reclassifying all trace calls as a negative result increased Xpert Ultra specificity and decreased its sensitivity. Reclassifying trace calls as negative in participants with a history of tuberculosis or repeating trace calls with the second result determining the ultimate classification, both resulted in sensitivity estimates close to those observed in the primary analysis with only slightly compromised specificity.

On 11 October 2018, we performed a literature search specifically for studies that evaluated Xpert Ultra, but did not identify any additional studies. Following this search and after the end date for data analysis, we identified one additional study (Berhanu 2018). Although not included in the main sections of this review, we provide a brief summary of this study here. Berhanu 2018 compared Xpert MTB/RIF and Xpert Ultra in 237 participants with presumptive tuberculosis who were evaluated at three outpatient clinics in South Africa. Similar to the results in Dorman 2018, this multicentre study found that Xpert Ultra yielded higher sensitivity at $89 \%$ (78\% to $96 \%$ ), compared to Xpert MTB/RIF sensitivity of $82 \%$ (70\% to $91 \%$ ), but lower specificity at $96 \%$ (92\% to $98 \%$ ) compared to Xpert MTB/ RIF specificity of $100 \%$ ( $98 \%$ to $100 \%$ ). Importantly, in both studies, Xpert Ultra had superior sensitivity for smear-negative tuberculosis: in Dorman 2018, Xpert Ultra sensitivity was 63\% (54\% to $71 \%$ ) versus Xpert MTB/RIF 46\% (37\% to 55\%); and in Berhanu 2018, Xpert Ultra sensitivity was $65 \%$ (38\% to $86 \%$ ) versus Xpert MTB/RIF $41 \%$ (18\% to $67 \%)$. In both studies, Xpert Ultra's increased sensitivity for smear-negative tuberculosis was accompanied by decreased specificity, $96 \%$ in both studies, versus Xpert MTB/RIF specificity of 98\% in Dorman 2018 and 100\% in Berhanu 2018. In addition, in Dorman 2018, in HIV-positive participants Xpert Ultra had higher sensitivity (90\%) than Xpert MTB/RIF (77\%), again accompanied by a decrease in specificity (Xpert Ultra specificity of $96 \%$ versus Xpert MTB/RIF specificity of 99\%). Xpert Ultra and Xpert MTB/RIF had similar accuracy for rifampicin resistance. As Xpert Ultra is rolled out globally, these differences in accuracy may have important ramifications depending on tuberculosis prevalence (Kendall 2017).
Our prespecified subgroup analyses included an assessment of whether Xpert MTB/RIF accuracy differs by the setting in which the test was performed. i.e. point of care or peripheral settings compared with central and intermediate laboratories. Theron 2014a found no difference in Xpert MTB/RIF accuracy when it was performed by trained nurses in a primary care setting compared to performance by laboratory technicians at a centralised facility. When we compared findings from studies by test setting, we found the pooled point estimates of Xpert MTB/RIF sensitivity and specificity to be lower in peripheral settings than in central and intermediate laboratories. However, there was considerable overlap in the credible intervals of these estimates and there is insufficient evidence to suggest a difference in Xpert MTB/RIF accuracy by setting. One of the confounding factors may be participant spectrum, the direction of which we cannot predict with certainty.

We acknowledge that patient health outcomes are clearly important to patients, to decision-makers, and the wider tuberculosis community. We could not, however, systematically address outcomes in addition to diagnostic accuracy, as they would have required a different methodology. Nonetheless, we are aware of seven trials that have examined the impact of Xpert MTB/RIF on mortality in relation to smear microscopy or diagnostic algorithms reflective of usual practice (Calligaro 2015; Churchyard 2015; Cox 2014; Mupfumi 2014; Ngwira 2019; Theron 2014a; Trajman 2015). All of these trials were conducted in routine healthcare settings. However, only two of these trials have shown a statistically significant impact on mortality (Ngwira 2019; Trajman 2015). Ngwira 2019 reported a significant impact on all-cause mortality in people with clinically advanced HIV when Xpert MTB/RIF testing at point of care was compared to LED microscopy among newly-diagnosed HIV-positive adults with presumptive tuberculosis in primary health clinics in Malawi, with an incidence rate ratio (RR) of $0.43 \%$ (95\% $\mathrm{Cl} 0.22 \%$ to $0.87 \%$ ). Trajman 2015 reported a lower tuberculosis-attributed death rate in the Xpert arm compared to the smear microscopy arm (2.3\% versus $3.8 \%)$ among adults with presumptive tuberculosis in primary health clinics in Brazil. In particular, this trial showed an association between HIV positivity and increased risk of tuberculosis-attributed death: adjusted odds ratio (aOR) 14.1 (95\% Cl 9.1\% to $26.5 \%$ ), and a $35 \%$ reduction in tuberculosis-attributed death by Xpert when adjusted for HIV status and age group; OR 0.65 (95\% Cl 0.44\% to 0.97\%) (Trajman 2015).

Reasons that have been proposed to explain the lack of evidence for Xpert MTB/RIF's impact on mortality include the following: low statistical power; a limited focus on populations most likely to benefit from Xpert MTB/RIF testing, such as people with rifampicin resistance; high rates of empirical treatment; loss of patients to follow-up; and health system weaknesses (Auld 2016a; Boyles 2017; Schumacher 2016; Theron 2014c). At the time of this writing, Haraka and colleagues are carrying out a Cochrane Review to assess the impact of Xpert MTB/RIF on health outcomes (Haraka 2018).

Early detection of tuberculosis and rifampicin resistance may not lead to improved patient outcomes if the test result is not linked to appropriate treatment and other healthcare services. In a recent editorial, Pai 2018 argues that introducing a new diagnostic tool such as Xpert MTB/RIF into a fragmented healthcare system and expecting to find improved impact on patient health is unrealistic. Rather, changes in many or all steps in the healthcare cascade are needed (Pai 2018). They propose a patient-centred approach to assessing the impact of an innovation in patient health by mapping 
the point in the healthcare cascade where the diagnostic tool is in troduced and identifying barriers to its effectiveness. In addition, the use of well-designed implementation research should make it possible to examine assumptions about how the new tool will work and its impact on endpoints throughout the healthcare cascade (Pai 2018).

Regarding resource requirements, the WHO convened a Guideline Development Group meeting by webinar specifically to review economic analyses on the use of Xpert MTB/RIF as the initial diagnostic test for all persons with tuberculosis signs and symptoms globally, and as an initial test in the 30 high tuberculosis burden countries. A review identified 15 cost-effectiveness studies, most of which took place in sub-Saharan Africa. Twelve studies found the use of Xpert MTB/RIF to be cost-effective in their setting and three studies (in India, Malawi, and South Africa) found the use of the test to be cost or cost-effectiveness neutral. The Guideline Development Group judged the requirements to implement Xpert MTB/RIF as being large (moderate-certainty evidence of resource requirements), and judged cost effectiveness probably to be in favour of the introduction of Xpert MTB/RIF. The group decided that there was insufficient evidence to change the strength of the recommendation for the use of Xpert MTB/RIF as the initial diagnostic test for all persons with signs and symptoms of tuberculosis from conditional to strong. With respect to the certainty of evidence, guideline members raised concerns about the lack of internationally recognized thresholds for cost effectiveness and affordability, limiting the interpretation of data about cost effectiveness or affordability at the country level, as well as the difficulty of making recommendations globally when evidence varies by setting (WHO 2016a).

Since the WHO recommended the use of Xpert MTB/RIF, country-level policy-makers have been making decisions about adoption and scale-up. The uptake has been much faster than for any other tuberculosis technology recommended by the WHO over the last 10 years. A recent survey of market penetration of Xpert MTB/ RIF in high tuberculosis burden countries found greater use of Xpert MTB/RIF compared to smear microscopy for tuberculosis diagnosis (Cazabon 2018).

This review represents the most comprehensive review of the diagnostic accuracy of Xpert MTB/RIF and Xpert Ultra, and provides evidence that may help countries to make decisions about scaling up the tests for programmatic management of tuberculosis and drugresistant tuberculosis. Although the information in this review will help to inform such decisions, other factors such as resource requirements and feasibility (including stable electrical power supply, temperature control, and maintenance of the cartridge modules) will also be important considerations.

\section{Application of the meta-analysis to a hypothetical cohort}

Summary of findings 1 and Summary of findings 2 summarize the findings of the review by applying the results to a hypothetical cohort of 1000 individuals with presumptive PTB or rifampicin resistance. We present several different scenarios. For Xpert MTB/RIF and Xpert Ultra for detection of PTB, we used prevalences of tuberculosis of $1 \%, 10 \%$, and $30 \%$. For detection of rifampicin resistance, we used prevalences of rifampicin resistance of $5 \%, 10 \%$, and $15 \%$ (5\% is estimated to be equivalent to the upper limit for rifampicin resistance prevalence in new cases; $15 \%$ is estimated to be the lower limit for rifampicin resistance prevalence among previously-treated cases). The consequences of false-positive results are patient anxiety, morbidity from additional testing and unnecessary treatment, and possible delay in further diagnostic evaluation. The consequences of false-negative results are increased risk of patient morbidity and mortality, and continued risk of community transmission of tuberculosis.

\section{Strengths and weaknesses of the review}

\section{Completeness of evidence}

The findings in this review are based on comprehensive searching, strict inclusion criteria, and standardized data extraction. This review includes a total of 95 studies. For Xpert MTB/RIF for detection of PTB, we included 86 studies involving 42,091 participants. For Xpert MTB/RIF for detection of rifampicin resistance, we included 57 studies involving 8287 participants. For the diagnostic accuracy of Xpert Ultra, we identified only one study. We had repeated correspondence with study authors to obtain additional data and information that was missing from the papers. The search strategy included studies published in all languages. Although we may have missed some studies despite the comprehensive search, as this was a large review, it is unlikely that the findings would have changed.

\section{Accuracy of the reference standards used}

Culture is regarded as the best available reference standard for active tuberculosis disease and was the reference standard for tuberculosis in this review. We considered the type of culture used in the included studies because liquid culture is more sensitive than solid culture (American Thoracic Society 2000). Most studies did use liquid culture or a combination of solid and liquid culture; only 13 of the 70 studies with unselected participants (19\%) exclusively used solid culture. Phenotypic culture-based DST methods using WHOrecommended critical concentrations (WHO Policy DST 2008) and MTBDRplus, a WHO-recommended test, were the reference standards for rifampicin resistance. Concerning the former, the WHO is currently reviewing the critical concentration to recommend for rifampicin resistance testing. Concerning the latter, only four of the 57 studies (7\%) used MTBDRplus alone as the reference standard.

\section{Quality and quality of reporting of the included studies}

Most studies used consecutive selection of participants and interpreted the reference standard results without knowledge of index test results. Xpert MTB/RIF and Xpert Ultra results are generated automatically, without requiring subjective interpretation. In general, studies were fairly well reported, although we corresponded with many authors for additional data and missing information. We encourage authors of future studies to follow the recommendations in the STARD statement to improve the quality of reporting (Bossuyt 2015).

\section{Interpretability of subgroup analyses}

We investigated potential sources of heterogeneity in different subgroups and settings. For tuberculosis detection, the test had higher sensitivity in smear-positive and HIV-negative participants. Generally, we found increased sensitivity in settings with higher tuberculosis prevalence (culture-confirmed tuberculosis cases in the study) and similar or slightly lower specificity.

\section{Comparison with other systematic reviews}

We are aware of 10 systematic reviews previously published that estimated diagnostic accuracy of Xpert MTB/RIF for PTB and rifampicin resistance in adults (Table 5). In these reviews, summa- 
ry sensitivities ranged from $67 \%$ (limited to smear-negative specimens) to $90 \%$ (in our review: $85 \%$ ) and summary specificities $97 \%$ to $99 \%$ (in our review: $98 \%$ ).

Compared with previous systematic reviews, our review extended the date of the search for potential studies for inclusion. Our strict inclusion criteria, for example, including only studies that used culture as the reference standard and excluding case-control studies, meant that some of the studies included in other reviews were excluded from our review.

\section{Completeness and relevance of the review}

This review included studies using all four generations of Xpert ( $G 1$, G2, G3, G4 cartridges) and the newest version, Xpert Ultra, although we identified only one study with Xpert Ultra. A Cochrane Review on Xpert MTB/RIF for extrapulmonary tuberculosis (including one study with Xpert Ultra) was recently published (Kohli 2018). This review found that in people with presumptive extrapulmonary tuberculosis, Xpert MTB/RIF may be helpful in confirming the diagnosis. Xpert MTB/RIF sensitivity varied across different extrapulmonary specimens, while for most specimens specificity was high. In addition, Xpert MTB/RIF was accurate for detection of rifampicin resistance (Kohli 2018). A Cochrane Review on Xpert MTB/RIF and Xpert Ultra for active tuberculosis in children is underway.

\section{Applicability of findings to the review question}

For detection of PTB, most studies evaluated sputum specimens submitted by participants with presumptive tuberculosis, and ran the test in primary care facilities and local hospitals. Hence, for most studies, the participant characteristics and settings matched our review question. For detection of rifampicin resistance, we had low concern in $46 \%$ of studies and high concern in only $7 \%$ of studies. However, in nearly half of the studies (47\%) the clinical setting was not reported or there was insufficient information to make a decision.

\section{AUTHORS' CONCLUSIONS}

\section{Implications for practice}

We found Xpert MTB/RIF to be sensitive and specific for detection of PTB and rifampicin resistance, findings which are consistent with those reported previously. Xpert MTB/RIF was more sensitive for tuberculosis in smear-positive than smear-negative participants, and HIV-negative than HIV-positive participants. Compared with Xpert MTB/RIF, Xpert Ultra had higher sensitivity and lower specificity for tuberculosis detection and similar sensitivity and specificity for rifampicin resistance detection (one study). Xpert MTB/RIF and Xpert Ultra provide accurate results and can allow rapid initiation of treatment for multidrug-resistant tuberculosis. The ongoing use of Xpert MTB/RIF or Xpert Ultra in tuberculosis programmes in high tuberculosis burden settings, as well as use in primary care clinics where the test provides the opportunity to begin treatment promptly, will contribute evidence on whether its use leads to improvements in patient health.

\section{Implications for research}

Future studies should assess the diagnostic accuracy of Xpert UItra compared with other rapid tests for tuberculosis and drug resistance, especially in difficult-to-diagnose groups, i.e. children, people living with HIV, and those with extrapulmonary tuberculosis. Understanding the impact of Xpert Ultra in settings with differing prevalences of tuberculosis, in previously-treated individuals, with varying strategies for the classification of trace calls, and its impact on patient health outcomes will be important.

\section{ACK N OWLEDGEMENTS}

The Academic Editors are Professor Gerry Davies and Dr Mariska Leeflang.

The editorial base of the Cochrane Infectious Diseases Group (CIDG) is funded by UK aid from the UK government for the benefit of lowand middle-income countries (project number 300342-104). The views expressed do not necessarily reflect the UK government's official policies.

We thank all authors of the included studies for answering our questions and providing additional data. We are grateful to Vittoria Lutje, CIDG Information Specialist, for help with the search strategy. We thank Hojoon Sohn and Lorie Kloda who contributed to the original review version. We also acknowledge Edward Desmond, California State Department of Health, for his comments on alternative tuberculosis tests, and Ellen Jo Baron, Cepheid, for her comments on the index test. In addition, we thank Chris Gilpin for assisting with the laboratory classification scheme and Matteo Zignol for providing the prevalence rates for MDR-TB; both experts are with the Global TB Programme, WHO, Geneva; and Selcan Alptekin, Rothamsted Research, for translation assistance. Study data were collected and managed using REDCap electronic data capture tools hosted at University of Washington (supported by UL1 TR002319, KL2 TR002317, and TL1 TR002318 from NCATS/NIH). 


\section{R E F E R E N C E S}

\section{References to studies included in this review}

Adelman 2015 \{published data only\}

Adelman MW, Tsegaye M, Kempker RR, Alebachew T, Haile K, Tesfaye A, et al. Intensified tuberculosis case finding among HIV-infected persons using a WHO symptom screen and Xpert ${ }^{\circledR}$ MTB/RIF. International Journal of Tuberculosis and Lung Disease 2015;19(10):1197-203.

\section{Al-Darraji 2013 \{published data only\}}

Al-Darraji HA, Abd Razak H, Ng KP, Altice FL, Kamarulzaman A. The diagnostic performance of a single GeneXpert MTB/RIF assay in an intensified tuberculosis case finding survey among HIV-infected prisoners in Malaysia. PLoS One 2013;8(9):e73717.

\section{Ali 2017 \{published data only\}}

Ali RH, Ibrahim NY, Elegail AM, Eltohami NA, Ebraheem RS, Ahmed SF, et al. Evaluation of GeneXpert MTB/RIF and line probe assay for rapid diagnosis of Mycobacterium tuberculosis in Sudanese pulmonary TB patients. Asian Pacific Journal of Tropical Disease 2017;7(7):426-9.

\section{Atwebembeire 2016 \{published data only\}}

Atwebembeire J, Orikiriza P, Bonnet M, Atwine D, Katawera V, Nansumba M, et al. Xpert ${ }^{\circledR}$ MTB/RIF for detection of Mycobacterium tuberculosis from frozen string and induced sputum sediments. International Journal of Tuberculosis and Lung Disease 2016;20(8):1113-7.

\section{Balcells 2012 \{published data only\}}

Balcells ME, García P, Chanqueo L, Bahamondes L, Lasso M, Gallardo AM, et al. Rapid molecular detection of pulmonary tuberculosis in HIV-infected patients in Santiago, Chile. International Journal of Tuberculosis and Lung Disease 2012;16(10):1349-53.

\section{Balcha 2014 \{published data only\}}

Balcha TT, Sturegard E, Winqvist N, Skogmar S, Reepalu A, Jemal ZH, et al. Intensified tuberculosis case-finding in HIVpositive adults managed at Ethiopian health centers: diagnostic yield of Xpert MTB/RIF compared with smear microscopy and liquid culture. PLoS One 2014;9(1):e85478.

\section{Barmankulova 2015 \{published data only\}}

Barmankulova A, Higuchi M, Sarker MA, Alim MA, Hamajima N. Tuberculosis and rifampicin resistance among migrants in Kyrgyzstan: detection by a new diagnostic test. Nagoya Journal of Medical Science 2015;77(1-2):41-9.

\section{Barnard 2015 \{published data only\}}

Barnard DA, Irusen EM, Bruwer JW, Plekker D, Whitelaw AC, Deetlefs JD, et al. The utility of Xpert MTB/RIF performed on bronchial washings obtained in patients with suspected pulmonary tuberculosis in a high prevalence setting. $B M C$ Pulmonary Medicine 2015;15:103.

\section{Bates 2013a \{published data only\}}

Bates M, Ahmed Y, Chilukutu L, Tembo J, Cheelo B, Sinyangwe S, et al. Use of the Xpert ${ }^{\circledR}$ MTB/RIF assay for diagnosing pulmonary tuberculosis comorbidity and multidrug-resistant TB in obstetrics and gynaecology inpatient wards at the University Teaching Hospital, Lusaka, Zambia. Tropical Medicine \& International Health 2013;18(9):1134-40.

\section{Bjerrum 2016 \{published data only\}}

Bjerrum S, Oliver-Commey J, Kenu E, Lartey M, Newman MJ, Addo KK, et al. Tuberculosis and non-tuberculous mycobacteria among HIV-infected individuals in Ghana. Tropical Medicine \& International Health 2016;21(9):1181-90.

\section{Boehme 2010 \{published data only\}}

Boehme CC, Nabeta P, Hillemann D, Nicol MP, Shenai S, Krapp F, et al. Rapid molecular detection of tuberculosis and rifampin resistance. New England Journal of Medicine 2010;363(11):1005-15

\section{Boehme 2011 \{published data only\}}

Boehme CC, Nicol MP, Nabeta P, Michael JS, Gotuzzo E, Tahirli R, et al. Feasibility, diagnostic accuracy, and effectiveness of decentralised use of the Xpert MTB/RIF test for diagnosis of tuberculosis and multidrug resistance: a multicentre implementation study. Lancet 2011;377(9776):1495-505.

\section{Boum 2016 \{published data only\}}

Boum Y, Kim S, Orikiriza P, Acuna-Villaorduna C, Vinhas S, Bonnet $\mathrm{M}$, et al. Diagnostic accuracy of the small membrane filtration method for diagnosis of pulmonary tuberculosis in a high-HIV-prevalence setting. Journal of Clinical Microbiology 2016;54(6):1520-7.

\section{Calligaro 2015 \{published data only\}}

Calligaro GL, Theron G, Khalfey H, Peter J, Meldau R, Matinyenya $B$, et al. Burden of tuberculosis in intensive care units in Cape Town, South Africa, and assessment of the accuracy and effect on patient outcomes of the Xpert MTB/RIF test on tracheal aspirate samples for diagnosis of pulmonary tuberculosis: a prospective burden of disease study with a nested randomised controlled trial. Lancet Respiratory Medicine 2015;3(8):621-30.

\section{Calligaro 2017 \{published data only\}}

Calligaro GL, Zijenah LS, Peter JG, Theron G, Buser V, McNerney R, et al. Effect of new tuberculosis diagnostic technologies on community-based intensified case finding: a multicentre randomised controlled trial. Lancet Infectious Diseases 2017;17(4):441-50.

\section{Carriquiry 2012 \{published data only\}}

Carriquiry G, Otero L, González-Lagos E, Zamudio C, Sánchez E, Nabeta $P$, et al. A diagnostic accuracy study of Xpert ${ }^{\circledR}$ MTB/RIF in HIV-positive patients with high clinical suspicion of pulmonary tuberculosis in Lima, Peru. PLoS One 2012;7(9):e44626.

\section{Chaisson 2014 \{published data only\}}

Chaisson LH, Roemer M, Cantu D, Haller B, Millman AJ, Cattamanchi A, et al. Impact of GeneXpert MTB/RIF assay on triage of respiratory isolation rooms for inpatients with 
presumed tuberculosis: a hypothetical trial. Clinical Infectious Diseases 2014;59(10):1353-60.

\section{Chen 2017 \{published data only\}}

Chen C, Yang CG, Gao X, Lu ZZ, Tang FX, Cheng J, et al. Community-based active case finding for tuberculosis in rural western China: a cross-sectional study. International Journal of Tuberculosis and Lung Disease 2017;21(11):1134-9.

\section{Chew 2016 \{published data only\}}

Chew MY, Ng J, Cai HM, Lim TG, Lim TK. The clinical utility of Xpert MTB/RIF testing in induced sputum. International Journal of Tuberculosis and Lung Disease 2016;20(12):1668-70.

\section{Chikaonda 2017 \{published data only\}}

Chikaonda T, Nguluwe N, Barnett B, Gokhale RH, Krysiak R, Thengolose I, et al. Performance of Xpert ${ }^{\circledR}$ MTB/RIF among tuberculosis outpatients in Lilongwe, Malawi. African Journal of Laboratory Medicine 2017;6(2):464.

\section{Cowan 2017 \{published data only\}}

Cowan JF, Chandler AS, Kracen E, Park DR, Wallis CK, Liu E, et al. Clinical impact and cost-effectiveness of Xpert MTB/RIF testing in hospitalized patients with presumptive pulmonary tuberculosis in the United States. Clinical Infectious Diseases 2017;64(4):482-9.

\section{Davis 2014 \{published data only\}}

Davis JL, Kawamura LM, Chaisson LH, Grinsdale J, Benhammou J, Ho C, et al. Impact of GeneXpert MTB/RIF on patients and tuberculosis programs in a low-burden setting. a hypothetical trial. American Journal of Respiratory and Critical Care Medicine 2014;189(12):1551-9.

\section{Dorman 2018 \{published data only\}}

Dorman SE, Schumacher SG, Alland D, Nabeta P, Armstrong DT, King B, et al. Xpert MTB/RIF Ultra for detection of Mycobacterium tuberculosis and rifampicin resistance: a prospective multicentre diagnostic accuracy study. Lancet Infectious Diseases 2018;18(1):76-84.

\section{Friedrich 2011 \{published data only\}}

Friedrich SO, Venter A, Kayigire XA, Dawson R, Donald PR, Diacon AH. Suitability of Xpert MTB/RIF and genotype MTBDRplus for patient selection for a tuberculosis clinical trial. Journal of Clinical Microbiology 2011;49(8):2827-31.

\section{Geleta 2015 \{published data only\}}

Geleta DA, Megerssa YC, Gudeta AN, Akalu GT, Debele MT, Tulu K D. Xpert MTB/RIF assay for diagnosis of pulmonary tuberculosis in sputum specimens in remote health care facility. BMC Microbiology 2015;15:220.

\section{Hanif 2011 \{published data only\}}

Hanif SN, Eldeen HS, Ahmad S, Mokaddas E. GeneXpert ${ }^{\circledast}$ MTB/RIF for rapid detection of Mycobacterium tuberculosis in pulmonary and extra-pulmonary samples. International Journal of Tuberculosis and Lung Disease 2011;15(9):1274-5.

\section{Hanrahan 2013 \{published data only\}}

Hanrahan CF, Selibas K, Deery CB, Dansey H, Clouse K, Bassett J, et al. Time to treatment and patient outcomes among TB suspects screened by a single point-of-Care Xpert MTB/RIF at a primary care clinic in Johannesburg, South Africa. PLoS One 2013;8(6):e65421.

\section{Hanrahan 2014 \{published data only\}}

Hanrahan CF, Theron G, Bassett J, Dheda K, Scott L, Stevens W, et al. Xpert MTB/RIF as a measure of sputum bacillary burden. Variation by HIV status and immunosuppression. American Journal of Respiratory and Critical Care Medicine 2014;189(11):1426-34

\section{Helb 2010 \{published data only\}}

Helb D, Jones M, Story E, Boehme C, Wallace E, Ho K, et al. Rapid detection of Mycobacterium tuberculosis and rifampin resistance by use of on-demand, near-patient technology. Journal of Clinical Microbiology 2010;48(1):229-37.

\section{Henostroza 2016 \{published data only\}}

Henostroza G, Harris JB, Chitambi R, Siyambango M Turnbull ER, Maggard KR, et al. High prevalence of tuberculosis in newly enrolled HIV patients in Zambia: need for enhanced screening approach. International Journal of Tuberculosis and Lung Disease 2016;20(8):1033-9.

\section{Huang 2015 \{published data only\}}

Huang F, Dang L, Sun H, Yang H, Wu X. A study of the value of three molecular diagnostic techniques in the diagnosis of tuberculosis. Zhonghua Jie He He Hu Xi Za Zhi 2015;38(9):680-5.

Huh 2014 \{published data only\}

Huh HJ, Jeong BH, Jeon K, Koh WJ, Ki CS, Lee NY. Performance evaluation of the Xpert MTB/RIF assay according to its clinical application. BMC Infectious Diseases 2014;14:589.

\section{Jo 2016 \{published data only\}}

Jo YS, Park JH, Lee JK, Heo EY, Chung HS, Kim DK. Discordance between MTB/RIF and real-time tuberculosisspecific polymerase chain reaction assay in bronchial washing specimen and its clinical implications. PLoS One 2016;11(10):e0164923.

\section{Kawkitinarong 2017 \{published data only\}}

Kawkitinarong K, Suwanpimolkul G, Kateruttanakul P, Manosuthi W, Ubolyam S, Sophonphan J, et al. Real-life clinical practice of using the Xpert MTB/RIF assay in Thailand. Clinical Infectious Diseases 2017;64(suppl_2):S171-8.

\section{Kim CH 2015 \{published data only\}}

Kim CH, Hyun IG, Hwang YI, Kim DG, Lee CY, Lee MG, et al. Identification of Mycobacterium tuberculosis and rifampin resistance in clinical specimens using the Xpert MTB/RIF assay. Annals of Clinical and Laboratory Science 2015;45(1):32-8.

\section{Ko 2016 \{published data only\}}

Ko Y, Lee HK, Lee YS, Kim MY, Shin JH, Shim EJ, et al. Accuracy of Xpert $^{\circledR}$ MTB/RIF assay compared with AdvanSure TB/NTM realtime PCR using bronchoscopy specimens. International Journal of Tuberculosis and Lung Disease 2016;20(1):115-20. 
Kurbaniyazova 2017 \{published data only\}

Kurbaniyazova G, Joncevska M, Kalon S, Kalmambetova G, Mohr T, Toktogonova A, et al. Results of Xpert ${ }^{\circledR}$ MTB/RIF implementation in Kyrgyzstan. International Journal of Tuberculosis and Lung Disease 2017;21(3):333-7.

Kurbatova 2013 \{published data only\}

Kurbatova EV, Kaminski DA, Erokhin VV, Volchenkov GV, Andreevskaya SN, Chernousova LN, et al. Performance of Cepheid ${ }^{\circledR}$ Xpert MTB/RIF ${ }^{\circledR}$ and TB-Biochip ${ }^{\circledR}$ MDR in two regions of Russia with a high prevalence of drug-resistant tuberculosis. European Journal of Clinical Microbiology and Infectious Disease 2013;32(6):735-43.

\section{Kwak 2013 \{published data only\}}

Kwak N, Choi SM, Lee J, Park YS, Lee CH, Lee SM, et al. Diagnostic accuracy and turnaround time of the Xpert MTB/RIF assay in routine clinical practice. PloS One 2013;8(10):e77456.

\section{LaCourse 2016 \{published data only\}}

LaCourse SM, Cranmer LM, Matemo D, Kinuthia J, Richardson BA, John-Stewart G, et al. Tuberculosis case finding in HIV-Infected pregnant women in Kenya reveals poor performance of symptom screening and rapid diagnostic tests. Journal of Acquired Immune Deficiency Syndromes 2016;71(2):219-27.

\section{Lawn 2011 \{published data only\}}

Lawn SD, Brooks SV, Kranzer K, Nicol MP, Whitelaw A, Vogt M, et al. Screening for HIV-associated tuberculosis and rifampicin resistance before antiretroviral therapy using the Xpert MTB/RIF assay: a prospective study. PLoS Medicine 2011;8(7):e1001067.

\section{Lee 2013 \{published data only\}}

Lee HY, Seong MW, Park SS, Hwang SS, Lee J, Park YS, et al. Diagnostic accuracy of Xpert ${ }^{\circledast}$ MTB/RIF on bronchoscopy specimens in patients with suspected pulmonary tuberculosis. International Journal of Tuberculosis and Lung Disease 2013;17(7):917-21.

\section{Le Palud 2014 \{published data only\}}

Le Palud P, Cattoir V, Malbruny B, Magnier R, Campbell K, Oulkhouir $Y$, et al. Retrospective observational study of diagnostic accuracy of the Xpert ${ }^{\circledR}$ MTB/RIF assay on fiberoptic bronchoscopy sampling for early diagnosis of smear-negative or sputum-scarce patients with suspected tuberculosis. BMC Pulmonary Medicine 2014;14:137.

\section{Lippincott 2014 \{published data only\}}

Lippincott CK, Miller MB, Popowitch EB, Hanrahan CF, Van Rie A. Xpert MTB/RIF assay shortens airborne isolation for hospitalized patients with presumptive tuberculosis in the United States. Clinical Infectious Diseases 2014;59(2):186-92.

\section{Liu 2017 \{published data only\}}

Liu Z, Pan A, Wu B, Zhou L, He H, Meng Q, et al. Feasibility of a new model for early detection of patients with multidrugresistant tuberculosis in a developed setting of eastern China. Tropical Medicine \& International Health 2017;22(10):1328-33.

\section{Lorent 2015 \{published data only\}}

Lorent N, Kong C, Kim T, Sam S, Thai S, Colebunders R, et al. Systematic screening for drug-resistant tuberculosis with Xpert ${ }^{\circledR}$ MTB/RIF in a referral hospital in Cambodia. International Journal of Tuberculosis and Lung Disease 2015;19(12):1528-35.

\section{Luetkemeyer 2016 \{published data only\}}

Luetkemeyer AF, Firnhaber C, Kendall MA, Wu X, Mazurek GH, Benator DA, et al. Evaluation of Xpert MTB/RIF versus AFB smear and culture to identify pulmonary tuberculosis in patients with suspected tuberculosis from low and higher prevalence settings. Clinical Infectious Diseases 2016;62(9):1081-8.

\section{Makamure 2017 \{published data only\}}

Makamure B, Makumbirofa S, Bandason T, Leccese P, Mutetwa R, Robertson V, et al. A suggested algorithm for detection of multi drug-resistant tuberculosis in Zimbabwe. Journal of Infection in Developing Countries 2017;11(8):611-8.

\section{Mbelele 2017 \{published data only\}}

Mbelele PM, Aboud S, Mpagama SG, Matee MI. Improved performance of Xpert MTB/RIF assay on sputum sediment samples obtained from presumptive pulmonary tuberculosis cases at Kibong'oto infectious diseases hospital in Tanzania. BMC Infectious Diseases 2017;17(1):808.

\section{Meawed 2016 \{published data only\}}

Meawed TE, Shaker A. Assessment of diagnostic accuracy of Gene Xpert MTB/RIF in diagnosis of suspected retreatment pulmonary tuberculosis patients. Egyptian Journal of Chest Diseases and Tuberculosis 2016;65(3):637-41.

\section{Metcalfe 2015 \{published data only\}}

Metcalfe JZ, Makumbirofa S, Makamure B, Mutetwa R, Peñaloza RA, Sandy C, et al. Suboptimal specificity of Xpert MTB/RIF among treatment-experienced patients. European Respiratory Journal 2015;45(5):1504-6.

\section{Metcalfe 2016 \{published data only\}}

Metcalfe JZ, Makumbirofa S, Makamure B, Sandy C, Bara W, Mason P, et al. Xpert MTB/RIF detection of rifampin resistance and time to treatment initiation in Harare, Zimbabwe. International Journal of Tuberculosis and Lung Disease 2016;20(7):882-9.

\section{Meyer 2017 \{published data only\}}

Meyer AJ, Atuheire C, Worodria W, Kizito S, Katamba A, Sanyu I, et al. Sputum quality and diagnostic performance of GeneXpert MTB/RIF among smear-negative adults with presumed tuberculosis in Uganda. PLoS One 2017;12(7):e0180572.

\section{Mok 2016 \{published data only\}}

Mok Y, Tan TY, Tay TR, Wong HS, Tiew PY, Kam JW, et al. Do we need transbronchial lung biopsy if we have bronchoalveolar lavage Xpert ${ }^{\circledR}$ MTB/RIF?. International Journal of Tuberculosis and Lung Disease 2016;20(5):619-24.

\section{Mokaddas 2015 \{published data only\}}

Mokaddas E, Ahmad S, Eldeen HS, Al-Mutairi N. Discordance between Xpert MTB/RIF assay and Bactec MGIT 960 Culture System for detection of rifampin-resistant Mycobacterium 
tuberculosis isolates in a country with a low tuberculosis (TB) incidence. Journal of Clinical Microbiology 2015;53(4):1351-4.

\section{Mollel 2017 \{published data only\}}

Mollel EW, Chilongola JO, Mpagama SG, Kibiki GS. Evaluation of XpertMTB/RIF performance for diagnosis of tuberculosis among HIV positive patients in northern Tanzania. Tanzania Journal of Health Research 2017;19(1):1. [DOI: dx.doi.org/10.4314/ thrb.v19i1.1]

\section{Moure 2011 \{published data only\}}

Moure R, Muñoz L, Torres M, Santin M, Martín R, Alcaide F. Rapid detection of Mycobacterium tuberculosis complex and rifampin resistance in smear-negative clinical samples by use of an integrated real-time PCR method. Journal of Clinical Microbiology 2011;49(3):1137-9.

\section{Moussa 2016 \{published data only\}}

Moussa HS, Bayoumi FS, Ali AM. Evaluation of GeneXpert MTB/ RIF assay for direct diagnosis of pulmonary tuberculosis. Saudi Medical Journal 2016;37(10):1076-81.

\section{Mutingwende 2015 \{published data only\}}

Mutingwende I, Vermeulen U, Steyn F, Viljoen H, Grobler A. Development and evaluation of a rapid multiplex-PCR based system for Mycobacterium tuberculosis diagnosis using sputum samples. Journal of Microbiological Methods 2015;116:37-43.

\section{N'Guessan 2016 \{published data only\}}

N'Guessan Kouassi K, Riccardo A, Dutoziet Christian C, Andre G, Ferilaha C, Hortense SA, et al. Genotyping of mutations detected with GeneXpert. International Journal of Mycobacteriology 2016;5(2):142-7.

\section{Ngabonziza 2016 \{published data only\}}

Ngabonziza JC, Ssengooba W, Mutua F, Torrea G, Dushime A, Gasana M, et al. Diagnostic performance of smear microscopy and incremental yield of Xpert in detection of pulmonary tuberculosis in Rwanda. BMC Infectious Diseases 2016;16(1):660.

\section{Nikam 2014 \{published data only\}}

Nikam C, Kazi M, Nair C, Jaggannath M, Minoj M, Vinaya R, et al. Evaluation of the Indian TrueNAT micro RT-PCR device with GeneXpert for case detection of pulmonary tuberculosis. International Journal of Mycobacteriology 2014;3(3):205-10.

\section{Nliwasa 2016 \{published data only\}}

Nliwasa M, MacPherson P, Chisala P, Kamdolozi M, Khundi M, Kaswaswa K, et al. The sensitivity and specificity of LoopMediated Isothermal Amplification (LAMP) assay for tuberculosis diagnosis in adults with chronic cough in Malawi. PLoS One 2016;11(5):e0155101.

\section{Nosova 2013a \{published data only\}}

Nosova EY, Krasnova MA, Galkina KY, Makarova MV, Litvinov VI, Moroz AM. Comparative analysis of TB-Biochip, Xpert MTB/RIF, and GenoType MTBDRplus test systems for rapid determination of mutations responsible for drug resistance of M-tuberculosis complex (in sputum from patients in Moscow region). Molecular Biology (Moscow) 2013;47(2):236-41.

\section{O'Donnell 2015 \{published data only\}}

O'Donnell MR, Pym A, Jain P, Munsamy V, Wolf A, Karim F, et al. A novel reporter phage to detect tuberculosis and rifampin resistance in a high-HIV-burden population. Journal of Clinical Microbiology 2015;53(7):2188-94.

Park 2013 \{published data only\}

Park KS, Kim JY, Lee JW, Hwang YY, Jeon K, Koh WJ, et al. Comparison of the Xpert MTB/RIF and Cobas TaqMan MTB assays for detection of Mycobacterium tuberculosis in respiratory specimens. Journal of Clinical Microbiology 2013;51(10):3225-7.

Pimkina 2015 \{published data only\}

Pimkina E, Zablockis R, Nikolayevskyy V, Danila E, Davidaviciene $E$. The Xpert $^{\circledR}$ MTB/RIF assay in routine diagnosis of pulmonary tuberculosis: a multicentre study in Lithuania. Respiratory Medicine 2015;109(11):1484-9.

\section{Pinyopornpanish 2015 \{published data only\}}

Pinyopornpanish K, Chaiwarith R, Pantip C, Keawvichit R, Wongworapat K, Khamnoi P, et al. Comparison of Xpert MTB/ RIF assay and the conventional sputum microscopy in detecting Mycobacterium tuberculosis in northern Thailand. Tuberculosis Research and Treatment 2015;2015:571782.

\section{Rachow 2011 \{published data only\}}

Rachow A, Zumla A, Heinrich N, Rojas-Ponce G, Mtafya B, Reither K, et al. Rapid and accurate detection of Mycobacterium tuberculosis in sputum samples by Cepheid Xpert MTB/RIF assay--a clinical validation study. PLoS One 2011;6(6):e20458.

\section{Reddy 2017 \{published data only\}}

Reddy S, Ntoyanto S, Sakadavan Y, Reddy T, Mahomed S, Dlamini M, et al. Detecting Mycobacterium tuberculosis using the loop-mediated isothermal amplification test in South Africa. International Journal of Tuberculosis and Lung Disease 2017;21(10):1154-60.

\section{Reechaipichitkul 2017 \{published data only\}}

Reechaipichitkul W, Suleesathira T, Chaimanee P. Comparison of GeneXpert MTB/RIF assay with conventional AFB smear for diagnosis of pulmonary tuberculosis in northeastern Thailand. Southeast Asian Journal of Tropical Medicine and Public Health 2017;48(2):313-21.

\section{Rice 2017 \{published data only\}}

Rice JP, Seifert M, Moser KS, Rodwell TC. Performance of the Xpert MTB/RIF assay for the diagnosis of pulmonary tuberculosis and rifampin resistance in a low-incidence, highresource setting. PLoS One 2017;12(10):e0186139.

\section{Safianowska 2012 \{published data only\}}

Safianowska A, Walkiewicz R, Nejman-Gryz P, GrubekJaworska $\mathrm{H}$. Two selected commercially based nucleic acid amplification tests for the diagnosis of tuberculosis. Pneumonologia Alergologia Polska 2012;80(1):6-12.

\section{Sah 2017 \{published data only\}}

Sah AK, Joshi B, Khadka D, Gupta BP, Adhikari A, Singh SK, et al. Comparative study of GeneXpert MTB/RIF assay and 
Multiplex PCR assay for direct detection of Mycobacterium tuberculosis in suspected pulmonary tuberculosis patients. Current Microbiology 2017;74(9):1026-32.

\section{Scott 2011 \{published data only\}}

Scott LE, McCarthy K, Gous N, Nduna M, Van Rie A, Sanne I, et al. Comparison of Xpert MTB/RIF with other nucleic acid technologies for diagnosing pulmonary tuberculosis in a high HIV prevalence setting: a prospective study. PLoS Medicine 2011;8(7):e1001061.

\section{Scott 2017 \{published data only\}}

Scott L, David A, Noble L, Nduna M, Da Silva P, Black A, et al. Performance of the Abbott RealTime MTB and MTB RIF/INH assays in a setting of high tuberculosis and HIV coinfection in South Africa. Journal of Clinical Microbiology 2017;55(8):2491-501.

\section{Shao 2017 \{published data only\}}

Shao Y, Peng H, Chen C, Zhu T, Ji M, Jiang W, et al. Evaluation of GeneXpert MTB/RIF for detection of pulmonary tuberculosis at peripheral tuberculosis clinics. Microbial Pathogenesis 2017;105:260-3.

\section{Sharma 2015 \{published data only\}}

Sharma SK, Kohli M, Yadav RN, Chaubey J, Bhasin D, Sreenivas V, et al. Evaluating the diagnostic accuracy of Xpert MTB/RIF assay in pulmonary tuberculosis. PLoS One 2015;10(10):e0141011.

\section{Shenai 2016 \{published data only\}}

Shenai S, Armstrong DT, Valli E, Dolinger DL, Nakiyingi L, Dietze R, et al. Analytical and clinical evaluation of the Epistem Genedrive assay for detection of Mycobacterium tuberculosis. Journal of Clinical Microbiology 2016;54(4):1051-7.

\section{Singh 2016 \{published data only\}}

Singh UB, Pandey P, Mehta G, Bhatnagar AK, Mohan A, Goyal V, et al. Genotypic, phenotypic and clinical validation of GeneXpert in extra-pulmonary and pulmonary tuberculosis in India. PLoS One 2016;11(2):e0149258.

\section{Sohn 2014 \{published data only\}}

Sohn H, Aero AD, Menzies D, Behr M, Schwartzman K, Alvarez GG, et al. Xpert MTB/RIF testing in a low tuberculosis incidence, high-resource setting: limitations in accuracy and clinical impact. Clinical Infectious Diseases 2014;58(7):970-6.

\section{Ssengooba 2014 \{published data only\}}

Ssengooba W, Nakiyingi L, Armstrong DT, Cobelens FG, Alland D, Manabe Y C, et al. Clinical utility of a novel molecular assay in various combination strategies with existing methods for diagnosis of HIV-related tuberculosis in Uganda. PLoS One 2014;9(9):e107595.

\section{Tadesse 2016 \{published data only\}}

Tadesse M, Aragaw D, Rigouts L, Abebe G. Increased detection of smear-negative pulmonary tuberculosis by GeneXpert MTB/ $\mathrm{RIF}^{\oplus}$ assay after bleach concentration. International Journal of Mycobacteriology 2016;5(2):211-8.

\section{Tang 2017 \{published data only\}}

Tang T, Liu F, Lu X, Huang Q. Evaluation of GeneXpert MTB/RIF for detecting Mycobacterium tuberculosis in a hospital in China. Journal of International Medical Research 2017;45(2):816-22.

\section{Theron 2011 \{published data only\}}

Theron G, Peter J, Van Zyl-Smit R, Mishra H, Streicher E, Murray S, et al. Evaluation of the Xpert MTB/RIF assay for the diagnosis of pulmonary tuberculosis in a high HIV prevalence setting. American Journal of Respiratory and Critical Care Medicine 2011;184(1):132-40.

\section{Theron 2013 \{published data only\}}

Theron G, Peter J, Meldau R, Khalfey H, Gina P, Matinyena B, et al. Accuracy and impact of Xpert MTB/RIF for the diagnosis of smear-negative or sputum-scarce tuberculosis using bronchoalveolar lavage fluid. Thorax 2013;68(11):1043-51.

\section{Theron 2014a \{published data only\}}

Theron G, Zijenah L, Chanda D, Clowes P, Rachow A, Lesosky M, et al. Feasibility, accuracy, and clinical effect of point-of-care Xpert MTB/RIF testing for tuberculosis in primary-care settings in Africa: a multicentre, randomised, controlled trial. Lancet 2014;383(9915):424-35.

\section{Tsuyuguchi 2017 \{published data only\}}

Tsuyuguchi K, Nagai H, Ogawa K, Matsumoto T, Morimoto K, Takaki A, et al. Performance evaluation of Xpert MTB/RIF in a moderate tuberculosis incidence compared with TaqMan MTB and TRCRapid M.TB. Journal of Infection and Chemotherapy 2017;23(2):101-6.

\section{Van Rie 2013 \{published data only\}}

Van Rie A, Page-Shipp L, Hanrahan CF, Schnippel K, Dansey H, Bassett J, et al. Point-of-care Xpert ${ }^{\circledR}$ MTB/RIF for smearnegative tuberculosis suspects at a primary care clinic in South Africa. International Journal of Tuberculosis and Lung Disease 2013;17(3):368-72.

\section{Walusimbi 2013a \{published data only\}}

Walusimbi S, Bwanga F, Costa AD, Haile M, Hoffner S, Joloba M. Evaluation of the Xpert MTB/Rif test, microscopic observation drug susceptibility test and nitrate reductase assay, for rapid and accurate diagnosis of smear-negative tuberculosis in HIV patients. International Journal of Mycobacteriology 2013;2(3):148-55.

\section{Williamson 2012 \{published data only\}}

Williamson DA, Basu I, Bower J, Freeman JT, Henderson G, Roberts SA. An evaluation of the Xpert MTB/RIF assay and detection of false-positive rifampicin resistance in Mycobacterium tuberculosis. Diagnostic Microbiology and Infectious Disease 2012;74(2):207-9.

\section{Yoon 2017 \{published data only\}}

Yoon C, Semitala FC, Atuhumuza E, Katende J, Mwebe S, Asege $L$, et al. Point-of-care C-reactive protein-based tuberculosis screening for people living with HIV: a diagnostic accuracy study. Lancet Infectious Diseases 2017;17(12):1285-92. 
Zeka 2011 \{published data only\}

Zeka AN, Tasbakan S, Cavusoglu C. Evaluation of the GeneXpert MTB/RIF assay for the rapid diagnosis of tuberculosis and detection of RIF-resistance in pulmonary and extrapulmonary specimens. Journal of Clinical Micobiology 2011;49(12):4138-41.

Zetola 2014 \{published data only\}

Zetola NM, Shin SS, Tumedi KA, Moeti K, Ncube R, Nicol M, et al. Mixed Mycobacterium tuberculosis complex infections and false-negative results for rifampin resistance by GeneXpert MTB/RIF are associated with poor clinical outcomes. Journal of Clinical Microbiology 2014;52(7):2422-9.

Zmak 2013 \{published data only\}

Zmak L, Jankovic M, Jankovic VK. Evaluation of Xpert MTB/RIF assay for rapid molecular diagnosis of tuberculosis in a twoyear period in Croatia. International Journal of Mycobacteriology 2013;2(3):179-82.

\section{References to studies excluded from this review}

\section{Acuna-Villaorduna 2017 \{published data only\}}

Acuna-Villaorduna C, Orikiriza P, Nyehangane D, White LF, Mwanga-Amumpaire J, Kim S, et al. Effect of previous treatment and sputum quality on diagnostic accuracy of Xpert ${ }^{\circledR}$ MTB/ RIF. International Journal of Tuberculosis and Lung Disease 2017;21(4):389-97.

\section{Ade 2016 \{published data only\}}

Ade S, Adjibode O, Wachinou P, Toundoh N, Awanou B, Agodokpessi G, et al. Characteristics and treatment outcomes of retreatment tuberculosis patients in Benin. Tuberculosis Research and Treatment 2016;2016:1468631. [DOI: $10.1155 / 2016 / 1468631]$

\section{Adelman 2014 \{published data only\}}

Adelman MW, Tsegaye M, Kempker R, Abeje T, Tesfaye A, Aseffa A, et al. Enhanced active TB case finding among people living with hiv: impact of a rapid molecular test (XPERT MTB/ RIF). Journal of Investigative Medicine 2014;62(2):570.

\section{Agizew 2017 \{published data only\}}

Agizew T, Basotli J, Alexander H, Boyd R, Letsibogo G, Auld A, et al. Higher-than-expected prevalence of non-tuberculous mycobacteria in HIV setting in Botswana: implications for diagnostic algorithms using Xpert MTB/RIF assay. PLoS One 2017;12(12):e0189981. [DOI: 10.1371/journal.pone.0189981]

\section{Agrawal 2016 \{published data only\}}

Agrawal M, Bajaj A, Bhatia V, Dutt S. Comparative study of GeneXpert with ZN stain and culture in samples of suspected pulmonary tuberculosis. Journal of Clinical and Diagnostic Research 2016;10(5):DC09-12.

\section{Alame-Emane 2017 \{published data only\}}

Alame-Emane AK, Pierre-Audigier C, Aboumegone-Biyogo OC, Nzoghe-Mveang A, Cadet-Daniel V, Sola C, et al. Use of GeneXpert remnants for drug resistance profiling and molecular epidemiology of tuberculosis in Libreville, Gabon. Journal of Clinical Microbiology 2017;55(7):2105-15.

\section{Al-Ateah 2012 \{published data only\}}

Al-Ateah SM, Al-Dowaidi MM, El-Khizzi NA. Evaluation of direct detection of Mycobacterium tuberculosis complex in respiratory and non-respiratory clinical specimens using the Cepheid Gene Xpert ${ }^{\circledR}$ system. Saudi Medical Journal 2012;33(10):1100-5.

Albay 2016 \{published data only\}

Albay A, Guney M, Tekin K, Kisa O, Sig AK. Evaluation of the GeneXpert MTB/RIF assay for early diagnosis of tuberculosis and detection of rifampicin resistance in pulmonary and extrapulmonary specimens. Cukurova Medical Journal 2016;41(3):548-53.

\section{Al-Darraji 2016 \{published data only\}}

Al-Darraji HA, Altice FL, Kamarulzaman A. Undiagnosed pulmonary tuberculosis among prisoners in Malaysia: An overlooked risk for tuberculosis in the community. Tropical Medicine \& International Health 2016;21(8):1049-58.

\section{Ali 2016 \{published data only\}}

Ali RM, Alsudani AA. Discordance between GeneXpert assay and conventional drug-susceptibility testing in detecting rifampicin-resistant tuberculosis: a perspective of the line probe assay. International Journal of Mycobacteriology 2016;5 Suppl 1:S193-4.

\section{Alland 2015 \{published data only\}}

Alland D, Rowneki M, Smith L, Ryan J, Chancellor M, Marie Simmons A, et al. Xpert MTB/RIF Ultra: A new near-patient TB test with sensitivity equal to culture. Topics in Antiviral Medicine 2015;23(E-1):37.

\section{Alnimr 2014 \{published data only\}}

Alnimr AM, Hassan MI. Potential of two nucleic acid amplification assays for quantifying mycobacterial load in respiratory and non-respiratory specimens: a prospective study. Diagnostic Microbiology and Infectious Disease 2014;78(3):237-41.

\section{Alvarez 2015 \{published data only\}}

Alvarez GG, Dyk DD, Desjardlns M, Yasseen AS 3rd, Aaron SD, Cameron DW, et al. The feasibility, accuracy, and impact of Xpert MTB/RIF testing in a remote aboriginal community in Canada. Chest 2015;148(3):767-73.

\section{Alvarez-Uria 2012 \{published data only\}}

Alvarez-Uria G, Azcona JM, Midde M, Naik PK, Reddy S, Reddy R. Rapid diagnosis of pulmonary and extrapulmonary tuberculosis in HIV-infected patients. Comparison of LED fluorescent microscopy and the GeneXpert MTB/RIF assay in a district hospital in India. Tuberculosis Research and Treatment 2012;2012:932862. [DOI: 10.1155/2012/932862]

\section{Alvis-Zakzuk 2017 \{published data only\}}

Alvis-Zakzuk NJ, Carrasquilla ML, Gomez VJ, Robledo J, Alvis-Guzman NR, Hernandez JM. [Diagnostic accuracy of three technologies for the diagnosis of multi-drug resistant tuberculosis] [Precisión diagnóstica de tres pruebas moleculares para detectar la tuberculosis multirresistente]. Biomedica 2017;37(3):397-407. 
Andriani 2016 \{published data only\}

Andriani R, Burhan E, Isbaniah F, Atas Asri SD. Preliminary study of Xpert MTB/RIF assay for mycobacterium tuberculosis detection in new presumptive tuberculosis patients with negative sputum acid-fast bacilli. Respirology 2016;21 Suppl 3:197.

\section{Antonenka 2013 \{published data only\}}

Antonenka U, Hofmann-Thiel S, Turaev L, Esenalieva A, Abdulloeva M, Sahalchyk E, et al. Comparison of Xpert MTB/ RIF with ProbeTec ET DTB and COBAS TaqMan MTB for direct detection of $\mathrm{M}$. tuberculosis complex in respiratory specimens. BMC Infectious Diseases 2013;13:280. [DOI: 10.1186/1471-2334-13-280]

\section{Armand 2011 \{published data only\}}

Armand S, Vanhuls P, Delcroix G, Courcol R, Lemaître N. Comparison of the Xpert MTB/RIF test with an IS6110-TaqMan real-time PCR assay for direct detection of Mycobacterium tuberculosis in respiratory and nonrespiratory specimens. Journal of Clinical Microbiology 2011;49(5):1772-6.

\section{Asencio 2013 \{published data only\}}

Asencio Egea MA, Vaquero MH, Carranza Gonzalez R, Castellanos Monedero J, Franco Huerta M, Bravo Nieto JM, et al. Economic impact of the introduction of a technique for early detection of Mycobacterium tuberculosis Complex in clinical samples in a Spanish hospital. Revista Española de Salud Pública 2013;87(4):419-25.

\section{Aston 2016 \{published data only\}}

Aston SJ, Ho A, Jary H, Everett D, Mwandumba H, Heyderman RS, et al. Aetiology and outcome of communityacquired pneumonia in HIV-infected Malawian adults. Topics in Antiviral Medicine 2016;24(E-1):322.

\section{Atashi 2017 \{published data only\}}

Atashi S, Izadi B, Jalilian S, Madani SH, Farahani A, Mohajeri P. Evaluation of GeneXpert MTB/RIF for determination of rifampicin resistance among new tuberculosis cases in west and northwest Iran. New Microbes and New Infections 2017;19:117-20.

\section{Atehortua 2015 \{published data only\}}

Atehortua S, Ramirez F, Echeverri LM, Penata A, Ospina S. Xpert MTB/RIF test performance assay in respiratory samples at real work settings in a developing country. Biomedica 2015;35(1):125-30

\section{Atuhumuza 2016 \{published data only\}}

Atuhumuza E, Yoon C, Katende J, Asege L, Mwebe S, Andama A, et al. Intensified tuberculosis case-finding among people living with HIV: diagnostic yield of Xpert MTB/RIF, urine lipoarabinomannan and liquid culture. Journal of the International AIDS Society 2016;19:WEAB0202.

\section{Atwine 2015 \{published data only\}}

Atwine D, Nansumba M, Orikiriza P, Riera M, Nackers F, Kamara N, et al. Intra-gastric string test: an effective tool for diagnosing tuberculosis in adults unable to produce sputum. International Journal of Tuberculosis and Lung Disease 2015;19(5):558-64

Auld 2016b \{published data only\}

Auld SC, Moore BK, Kyle RP, Eng B, Nong K, Pevzner ES, et al. Mixed impact of Xpert ${ }^{\circledR}$ MTB/RIF on tuberculosis diagnosis in Cambodia. Public Health Action 2016;6(2):129-35.

\section{Aurin 2014 \{published data only\}}

Aurin TH, Munshi SK, Kamal SM, Rahman MM, Hossain MS, Marma T, et al. Molecular approaches for detection of the multidrug resistant tuberculosis (MDR-TB) in Bangladesh. PLoS One 2014;9(6):e99810.

\section{Avashia 2016 \{published data only\}}

Avashia S, Choubey S, Mishra S, Kharate A. To study the usefulness of CBNAAT (cartridge based nuclear acid amplification test) in BAL (bronchoalveolar lavage) samples in the diagnosis of smear-negative/non sputum producing patients with suspected tuberculosis. Journal of Evolution of Medical and Dental Sciences-JEMDS 2016;5(1):55-9.

\section{Ayala 2016 \{published data only\}}

Ayala G, Garay J, Aragon M, Decroo T, Zachariah R. Trends in tuberculosis notification and treatment outcomes in prisons: a country-wide assessment in El Salvador from 2009-2014. Revista Panamericana de Salud Pública 2016;39(1):38-43.

\section{Bablishvili 2015 \{published data only\}}

Bablishvili N, Tukvadze N, Avaliani Z, Blumberg HM, Kempker RR. A comparison of the Xpert ${ }^{\circledR}$ MTB/RIF and GenoType ${ }^{\circledR}$ MTBDRplus assays in Georgia. International Journal of Tuberculosis and Lung Disease 2015;19(6):676-8.

\section{Badal-Faesen 2017 \{published data only\}}

Badal-Faesen S, Firnhaber C, Kendall MA, Wu X, Grinsztejn B, Escada RO, et al. Impact of larger sputum volume on Xpert $^{\circledR}$ MTB/RIF assay detection of Mycobacterium tuberculosis in smear-negative individuals with suspected tuberculosis. Journal of Clinical Medicine 2017;6(8):pii: e78. [DOI: 10.3390/ jcm6080078]

\section{Bajrami 2016 \{published data only\}}

Bajrami R, Mulliqi G, Kurti A, Lila G, Raka L. Comparison of GeneXpert MTB/RIF and conventional methods for the diagnosis of tuberculosis in Kosovo. Journal of Infection in Developing Countries 2016;10(4):418-22.

Balcha 2014a \{published data only\}

Balcha TT, Winqvist N, Sturegard E, Skogmar S, Reepalu A, Jemal ZH, et al. Detection of lipoarabinomannan in urine for identification of active tuberculosis among HIV-positive adults in Ethiopian health centres. Tropical Medicine \& International Health 2014;19(6):734-42.

Banu 2014 \{published data only\}

Banu S, Rahman SM, Khan MS, Ferdous SS, Ahmed S, Gratz J, et al. Discordance across several methods for drug susceptibility testing of drug-resistant Mycobacterium tuberculosis isolates in a single laboratory. Journal of Clinical Microbiology 2014;52(1):156-63. 


\section{Barkham 2016 \{published data only\}}

Barkham T, Tang WY. GeneXpert-a state of the art commercial PCR assay, misses a fifth of tuberculosis cases. Annals of the Academy of Medicine Singapore 2016;45 (9 Supplement 1):S53.

\section{Barnard 2012 \{published data only\}}

Barnard M, Gey van Pittius NC, Van Helden PD, Bosman M, Coetzee $\mathrm{G}$, Warren RM. The diagnostic performance of the GenoType MTBDRplus version 2 line probe assay is equivalent to that of the Xpert MTB/RIF assay. Journal of Clinical Microbiology 2012;50(11):3712-6.

\section{Bates 2013b \{published data only\}}

Bates M, O'Grady J, Maeurer M, Tembo J, Chilukutu L, Chabala C, et al. Assessment of the Xpert MTB/RIF assay for diagnosis of tuberculosis with gastric lavage aspirates in children in sub-Saharan Africa: a prospective descriptive study. Lancet Infectious Diseases 2013;13(1):36-42.

\section{Biadglegne 2014 \{published data only\}}

Biadglegne F, Rodloff AC, Sack U. A first Insight into high prevalence of undiagnosed smear-negative pulmonary tuberculosis in northern Ethiopian prisons: implications for greater investment and quality control. PLOS One 2014;9(9):e106869.

\section{Bilgin 2016 \{published data only\}}

Bilgin K, Yanik K, Karadag A, Odabasi H, Tas H, Gunaydin M. Comparison of a real-time polymerase chain reaction-based system and Erlich-Ziehl-Neelsen method with culture in the identification of Mycobacterium tuberculosis. Turkısh Journal of Medical Sciences 2016;46(1):203-6.

\section{Bisognin 2018 \{published data only\}}

Bisognin F, Lombardi G, Lombardo D, Re MC, Dal Monte P. Improvement of Mycobacterium tuberculosis detection by Xpert MTB/RIF Ultra: A head-to-head comparison on Xpert-negative samples. PLoS One 2018;13(8):e0201934.

\section{Bjerrum 2015 \{published data only\}}

Bjerrum S, Kenu E, Lartey M, Newman M J, Addo KK, Andersen $A B$, et al. Diagnostic accuracy of the rapid urine lipoarabinomannan test for pulmonary tuberculosis among HIV-infected adults in Ghana-findings from the DETECT HIV-TB study. BMC Infectious Diseases 2015;15:407.

\section{Boakye-Appiah 2016 \{published data only\}}

Boakye-Appiah JK, Steinmetz AR, Pupulampu P, OforiYirenkyi S, Tetteh I, Frimpong M, et al. High prevalence of multidrug-resistant tuberculosis among patients with rifampicin resistance using GeneXpert Mycobacterium tuberculosis/rifampicin in Ghana. International Journal of Mycobacteriology 2016;5(2):226-30.

\section{Bojang 2016 \{published data only\}}

Bojang AL, Mendy FS, Tientcheu LD, Otu J, Antonio M, Kampmann B, et al. Comparison of TB-LAMP, GeneXpert MTB/ RIF and culture for diagnosis of pulmonary tuberculosis in The Gambia. Journal of Infection 2016;72(3):332-7.
Bonnet 2017 \{published data only\}

Bonnet M, San KC, Pho Y, Sok C, Dousset JP, Brant W, et al. Nontuberculous mycobacteria infections at a provincial reference hospital, Cambodia. Emerging Infectious Diseases 2017;23(7):1139-47.

\section{Bowles 2011 \{published data only\}}

Bowles EC, Freyée B, Van Ingen J, Mulder B, Boeree M J, Van Soolingen D. Xpert MTB/RIF ${ }^{\circledR}$, a novel automated polymerase chain reaction-based tool for the diagnosis of tuberculosis. International Journal of Tuberculosis and Lung Disease 2011;15(7):988-9.

\section{Bunsow 2014a \{published data only\}}

Bunsow E, Ruiz-Serrano MJ, Lopez Roa P, Kestler M, Viedma DG, Bouza E. Evaluation of GeneXpert MTB/RIF for the detection of Mycobacterium tuberculosis and resistance to rifampin in clinical specimens. Journal of Infection 2014;68(4):338-43.

\section{Capocci 2016 \{published data only\}}

Capocci S, Sewell J, Smith C, Cropley I, Bhagani S, Morris S, et al. Testing for TB in a contemporary UK HIV clinic-is it really worth it?. HIV Medicine 2016;17(Supplement 1):38-9.

\section{Causse 2011 \{published data only\}}

Causse M, Ruiz P, Gutiérrez-Aroca JB, Casal M. Comparison of two molecular methods for rapid diagnosis of extrapulmonary tuberculosis. Journal of Clinical Microbiology 2011;49(8):3065-7.

\section{Cavanaugh 2016 \{published data only\}}

Cavanaugh JS, Modi S, Musau S, McCarthy K, Alexander H, Burmen B, et al. Comparative yield of different diagnostic tests for tuberculosis among people living with HIV in western Kenya. PLoS One 2016;11(3):e0152364.

\section{Cayci 2017 \{published data only\}}

Cayci YT, Bilgin K, Coban AY, Birinci A, Durupinar B. An evaluation of false-positive rifampicin resistance on the Xpert MTB/RIF. Memórias do Instituto Oswaldo Cruz 2017;112(11):756-9.

\section{Celik 2015 \{published data only\}}

Celik C, Gozel MG, Bakici MZ, Berk S, Ozsahin SL, Gulturk E. Applicability of Xpert MTB/RIF assay for routine diagnosis of tuberculosis: a four-year single-center experience. Turkısh Journal of Medical Sciences 2015;45(6):1329-34.

\section{Chakravorty 2017 \{published data only\}}

Chakravorty S, Simmons AM, Rowneki M, Parmar H, Cao Y, Ryan J, et al. The new Xpert MTB/RIF Ultra: improving detection of Mycobacterium tuberculosis and resistance to rifampin in an assay suitable for point-of-care testing. Molecular Biology 2017;8(4):e00812-17. [DOI: 10.1128/mBio.00812-17]

\section{Chishty 2016 \{published data only\}}

Chishty S, Farooqi J, Shafqat Y, Shafiq S, Jabeen K, Hasan R. Performance of Xpert MTB/RIF assay from fluorescent acid fast stained slides. European Respiratory Journal. European Respiratory Society Annual Congress 2016;48(Suppl 60):PA2781. 
Ciftçi 2011 \{published data only\}

Ciftçi IH, Aslan MH, Aşik G. Evaluation of Xpert MTB/RIF results for the detection of Mycobacterium tuberculosis in clinical samples. Mikrobiyoloji bülteni 2011;45(1):43-7.

\section{Clouse 2012 \{published data only\}}

Clouse K, Page-Shipp L, Dansey H, Moatlhodi B, Scott L, Bassett J, et al. Implementation of Xpert MTB/RIF for routine point-of-care diagnosis of tuberculosis at the primary care level. South African Medical Journal 2012;102(10):805-7.

\section{Cross 2014 \{published data only\}}

Cross GB, Coles K, Nikpour M, Moore OA, Denholm J, $\mathrm{McBryde} E S$, et al. TB incidence and characteristics in the remote gulf province of Papua New Guinea: a prospective study. BMC Infectious Diseases 2014;14:93.

\section{Cross 2015 \{published data only\}}

Cross LJ, Anscombe C, McHugh TD, Abubakar I, Shorten RJ, Thorne N, et al. A rapid and sensitive diagnostic screening assay for detection of mycobacteria including Mycobacterium tuberculosis directly from sputum without extraction. International Journal of Bacteriology 2015;2015:593745.

\section{Dagnra 2015 \{published data only\}}

Dagnra AY, Mlaga KD, Adjoh K, Kadanga E, Disse K, Adekambi T. Prevalence of multidrug-resistant tuberculosis cases among HIV-positive and HIV-negative patients eligible for retreatment regimen in Togo using GeneXpert MTB/RIF. New Microbes and New Infections 2015;8:24-7.

\section{Daum 2015 \{published data only\}}

Daum LT, Peters RP, Fourie PB, Jonkman K, Worthy SA, Rodriguez JD, et al. Molecular detection of Mycobacterium tuberculosis from sputum transported in PrimeStore ${ }^{\circledR}$ from rural settings. International Journal of Tuberculosis and Lung Disease 2015;19(5):552-7.

\section{Deggim 2013 \{published data only\}}

Deggim V, Somoskovi A, Voit A, Bottger EC, Bloemberg GV. Integrating the Xpert MTB/RIF assay into a diagnostic workflow for rapid detection of Mycobacterium tuberculosis in a low-prevalence area. Journal of Clinical Microbiology 2013;51(7):2396-9.

\section{Dierberg 2016 \{published data only\}}

Dierberg KL, Dorjee K, Salvo F, Cronin WA, Boddy J, Cirillo D, et al. Improved detection of tuberculosis and multidrug-resistant tuberculosis among Tibetan refugees, India. Emerging Infectious Diseases 2016;22(3):463-8.

\section{Dorjee 2012 \{published data only\}}

Dorjee K, Salvo F, Dierberg KL. Xpert ${ }^{\circledR}$ MTB/RIF diagnosed disseminated smear-negative MDR-TB in a sub-district hospital in India. International Journal of Tuberculosis and Lung Disease 2012;16(11):1560-1.

\section{Dorman 2012 \{published data only\}}

Dorman SE, Chihota VN, Lewis JJ, Shah M, Clark D, Grant AD, et al. Performance characteristics of the Cepheid Xpert
MTB/RIF test in a tuberculosis prevalence survey. PLoS One 2012; 7(8):e43307.

Dowdy 2011 \{published data only\}

Dowdy DW, Cattamanchi A, Steingart KR, Pai M. Is scale-up worth it? Challenges in economic analysis of diagnostic tests for tuberculosis. PLoS Medicine 2011;8(7):e1001063.

Feasey 2013 \{published data only\}

Feasey NA, Banada PP, Howson W, Sloan DJ, Mdolo A, Boehme $\mathrm{C}$, et al. Evaluation of Xpert MTB/RIF for detection of tuberculosis from blood samples of HIV-infected adults confirms Mycobacterium tuberculosis bacteremia as an indicator of poor prognosis. Journal of Clinical Microbiology 2013;51(7):2311-6.

\section{Fernandez 2017 \{published data only\}}

Fernandez Sanchez M, Lasso JI, Canas A, Morantes Ariza C, Cortes G, Sanchez Duran L, et al. Evaluation of the operating characteristics of GeneXpert MTB/RIF at a national reference center: Hospital Universitario San Ignacio, Bogota, Colombia. American Journal of Respiratory and Critical Care Medicine. American Thoracic Society International Conference 2017;195:A2084.

\section{FIND 2011 \{published data only\}}

Foundation for Innovative Diagnostics. Performance of Xpert MTB/RIF Version G4 assay, Version and date: 1.0/30 Nov 2011, Project: 7210. www.stoptb.org/wg/gli/assets/documents/map/ findg4cartridge.pdf 2011 (accessed 8 May 2019):1-8.

\section{Fong 2017 \{published data only\}}

Fong A, Wei C, Chang AH, Kerndt PR, Shulman IA, Butler-Wu S. Evaluation of the Xpert MTB/RIF assay for the detection of tuberculosis in patients being evaluated for tuberculosis in a large public hospital in the United States. Laboratory Investigation 2017;97 Suppl 1:390A.

\section{Friedrich 2011a \{published data only\}}

Friedrich SO, Von Groote-Bidlingmaier F, Diacon AH. Xpert MTB/ RIF assay for the diagnosis of pleural tuberculosis. Journal of Clinical Microbiology 2011;49(12):4341-2.

\section{Gama de Andrade 2017 \{published data only\}}

Gama de Andrade TL, Gouget Ferreira Silvano RG, Pombo March MF, Coelho Soares EC, Couto Sant'anna C, Baroni Aurilio R. The Xpert MTB-RIF to diagnose tuberculosis in adolescents from Rio de Janeiro, Brazil. Pediatric Pulmonology 2017;52(Suppl 46):S164-5.

\section{Gelalcha 2017 \{published data only\}}

Gelalcha AG, Kebede A, Mamo H. Light-emitting diode fluorescent microscopy and Xpert MTB/RIF(R) assay for diagnosis of pulmonary tuberculosis among patients attending Ambo hospital, west-central Ethiopia. BMC Infectious Diseases 2017;17(1):613

\section{Gounder 2014 \{published data only\}}

Gounder A, Gounder S, Reid SA. Evaluation of the implementation of the Xpert(R) MTB/RIF assay in Fiji. Public Health Action 2014:4(3):179-83. 


\section{Griesel 2016 \{published data only\}}

Griesel R, Stewart A, Van Der Plas H, Sikhondze W, Rangaka M, Maartens $\mathrm{G}$, et al. A clinical prediction rule for the diagnosis of tuberculosis in seriously ill adults. Topics in Antiviral Medicine 2016;24 (E-1):309-10.

\section{Griesel 2017 \{published data only\}}

Griesel R, Stewart A, Van der Plas H, Sikhondze W, Rangaka MX, Nicol MP, et al. Optimizing tuberculosis diagnosis in HIV-infected inpatients meeting the criteria of seriously ill in the WHO algorithm. Clinical Infectious Diseases 2017;66(9):1419-26.

\section{Guenaoui 2016 \{published data only\}}

Guenaoui K, Harir N, Ouardi A, Zeggai S, Sellam F, Bekri F, et al. Use of GeneXpert Mycobacterium tuberculosis/rifampicin for rapid detection of rifampicin resistant Mycobacterium tuberculosis strains of clinically suspected multi-drug resistance tuberculosis cases. Annals of Translational Medicine 2016;4(9):168.

\section{Gupta 2014 \{published data only\}}

Gupta RK, Lawn SD, Booth H, Morris-Jones S. What is the role for Xpert ${ }^{\circledR}$ MTB/RIF in high-resource settings? Experience from a central London hospital. International Journal of Tuberculosis and Lung Disease 2014;18(11):1323-6.

\section{Gurbanova 2016 \{published data only\}}

Gurbanova E, Mehdiyev R, Blondal K, Tahirli R, Mirzayev F, Hillemann $D$, et al. Interpretation of indeterminate RIFsusceptibility results obtained by rapid molecular diagnostics test. European Respiratory Journal. European Respiratory Society Annual Congress 2016;48(Suppl 60):PA1907.

\section{Gurbanova 2017 \{published data only\}}

Gurbanova E, Mehdiyev R, Blondal K, Tahirli R, Mirzayev F, Hillemann $D$, et al. Mitigation of discordant rifampicinsusceptibility results obtained by Xpert Mycobacterium tuberculosis/Rifampicin and Mycobacterium Growth Indicator Tube. Microbial Drug Resistance 2017;23(8):1045-1052.

\section{Gursoy 2016 \{published data only\}}

Gursoy NC, Yakupogullari Y, Tekerekoglu MS, Otlu B. Evaluation of the diagnostic performance of Xpert MTB/RIF test for the detection of Mycobacterium tuberculosis and rifampin resistance in clinical samples. Mikrobiyoloji bülteni 2016;50(2):196-204.

\section{Habeenzu 2017 \{published data only\}}

Habeenzu C, Nakajima C, Solo E, Bwalya P, Kajino K, Miller M, et al. Evaluation of in-house loop-mediated isothermal amplification for tuberculosis diagnosis compared with Xpert MTB/RIF. Journal of Infection in Developing Countries 2017;11(6):440-4.

\section{Hanifa 2016 \{published data only\}}

Hanifa Y, Fielding KL, Chihota VN, Adonis L, Charalambous S, Karstaedt A, et al. Diagnostic accuracy of lateral flow urine LAM assay for TB screening of adults with advanced immunosuppression attending routine HIV care in South Africa. PLoS One 2016;11(6):e0156866.

\section{Heidebrecht 2016 \{published data only\}}

Heidebrecht CL, Podewils LJ, Pym AS, Cohen T, Mthiyane T, Wilson D. Assessing the utility of Xpert ${ }^{\circledR}$ MTB/RIF as a screening tool for patients admitted to medical wards in South Africa. Scientific Reports 2016;6:19391.

\section{Hillemann 2011 \{published data only\}}

Hillemann D, Rüsch-Gerdes S, Boehme C, Richter E. Rapid molecular detection of extrapulmonary tuberculosis by the automated GeneXpert MTB/RIF system. Journal of Clinical Microbiology 2011;49(4):1202-5.

\section{Hiza 2017 \{published data only\}}

Hiza H, Doulla B, Sasamalo M, Hella J, Kamwela L, Mhimbira F, et al. Preservation of sputum samples with cetylpyridinium chloride (CPC) for tuberculosis cultures and Xpert MTB/RIF in a low-income country. BMC Infectious Diseases 2017;17(1):542.

\section{Ho 2016 \{published data only\}}

Ho J, Nguyen PT, Nguyen TA, Tran KH, Nguyen S, Nguyen NV, et al. Reassessment of the positive predictive value and specificity of Xpert MTB/RIF: a diagnostic accuracy study in the context of community-wide screening for tuberculosis. Lancet Infectious Diseases 2016;16(9):1045-51.

\section{Horo 2017 \{published data only\}}

Horo K, N'Guessan R, Koffi MO, Kouame-N'Takpe N, Kone A, Samake K, et al. Use of the Xpert ${ }^{\circledR}$ MTB/RIF test in routine screening of new cases of pulmonary tuberculosis in an endemic area. Revue des Maladies Respiratoires 2017;34(7):749-57.

\section{Hu 2014 \{published data only\}}

Hu P, Bai L, Liu F, Ou X, Zhang Z, Yi S, et al. Evaluation of the Xpert MTB/RIF assay for diagnosis of tuberculosis and rifampin resistance in county-level laboratories in Hunan province, China. Chinese Medical Journal 2014;127(21):3744-50.

\section{Huang 2018 \{published data only\}}

Huang H, Zhang Y, Li S, Wang J, Chen J, Pan Z, et al. Rifampicin resistance and multidrug-resistant tuberculosis detection using Xpert MTB/RIF in Wuhan, China: a retrospective study. Microbial Drug Resistance 2018;24(5):675-9. [DOI: 10.1089/mdr.2017.0114]

\section{Huerga 2017 \{published data only\}}

Huerga H, Ferlazzo G, Bevilacqua P, Kirubi B, Ardizzoni E, Wanjala $S$, et al. Incremental yield of including determineTB LAM assay in diagnostic algorithms for hospitalized and ambulatory HIV-positive patients in Kenya. PLoS One 2017;12(1):e0170976. [DOI: 10.1371/journal.pone.0170976]

\section{Ioannidis 2010 \{published data only\}}

Ioannidis P, Papaventsis D, Nikolaou S, Karabela S, Konstantinidou E, Marinou I, et al. Tuberculosis resistance detection rate to the two main anti-TB drugs, isoniazid and rifampicin, using molecular techniques: Experience of the Hellenic National Reference Center for Mycobacteria. Acta Microbiologica Hellenica 2010;55:175-82. 
Ioannidis 2011 \{published data only\}

loannidis P, Papaventsis D, Karabela S, Nikolaou S, Panagi M, Raftopoulou E, et al. Cepheid GeneXpert MTB/RIF assay for Mycobacterium tuberculosis detection and rifampin resistance identification in patients with substantial clinical indications of tuberculosis and smear-negative microscopy results. Journal of Clinical Microbiology 2011;49(8):3068-70.

\section{Iram 2015 \{published data only\}}

Iram S, Zeenat A, Hussain S, Wasim Yusuf N, Aslam M. Rapid diagnosis of tuberculosis using Xpert MTB/RIF assay - report from a developing country. Pakistan Journal of Medical Sciences 2015;31(1):105-10.

\section{Jafari 2013 \{published data only\}}

Jafari C, Ernst M, Kalsdorf B, Lange C. Comparison of molecular and immunological methods for the rapid diagnosis of smearnegative tuberculosis. International Journal of Tuberculosis and Lung Disease 2013;17(11):1459-65.

\section{Jing 2017 \{published data only\}}

Jing H, Lu ZM, Deng YF, Gao DC, Li L, Graviss EA, et al. Evaluation of Xpert MTB/RIF in detection of pulmonary and extrapulmonary tuberculosis cases in China. International Journal of Clinical and Experimental Pathology 2017;10(4):4847-51.

\section{Jipa 2016 \{published data only\}}

Jipa R, Manea E, Cernat R, Iringo K, Vat AA, Arbune M, et al. Drug-resistant tuberculosis in HIV infected patients. BMC Infectious Diseases. 12th Scientific Days of the National Institute for Infectious Diseases "Prof. Dr. Matei Bals" and the 12th National Infectious Diseases Conference. Bucharest 2016;16(4):A107.

\section{Jones-Lopez 2014 \{published data only\}}

Jones-Lopez E, Manabe YC, Palaci M, Kayiza C, Armstrong D, Nakiyingi L, et al. Prospective cross-sectional evaluation of the small membrane filtration method for diagnosis of pulmonary tuberculosis. Journal of Clinical Microbiology 2014;52(7):2513-20.

\section{Kang 2016 \{published data only\}}

Kang JY, Hyung Woo K, Sanghoon J, Jaeha L, Shinyoung K, Chan Kwon $P$, et al. Clinical features of discordant result between molecular and phenotypic susceptibility tests in tuberculosis patients. Respirology 2016;21(Suppl 3):200.

\section{Kaur 2016 \{published data only\}}

Kaur R, Kachroo K, Sharma JK, Vatturi SM, Dang A. Diagnostic accuracy of Xpert test in tuberculosis detection: a systematic review and meta-analysis. Journal of Global Infectious Diseases 2016;8(1):32-40.

\section{Kayigire 2013 \{published data only\}}

Kayigire XA, Friedrich SO, Venter A, Dawson R, Gillespie SH, Boeree MJ, et al. Direct comparison of Xpert MTB/RIF assay with liquid and solid mycobacterial culture for quantification of early bactericidal activity. Journal of Clinical Microbiology 2013;51(6):1894-8.
Kelly-Cirino 2017 \{published data only\}

Kelly-Cirino CD, Musisi E, Byanyima P, Kaswabuli S, Andama A, Sessolo A, et al. Investigation of OMNIgene.SPUTUM performance in delayed tuberculosis testing by smear, culture, and Xpert MTB/RIF assays in Uganda. Journal of Epidemiology and Global Health 2017;7(2):103-9.

Kerkhoff 2013 \{published data only\}

Kerkhoff AD, Wood R, Lowe D M, Vogt M, Lawn SD. Blood neutrophil counts in HIV-infected patients with pulmonary tuberculosis: association with sputum mycobacterial load. PLOS ONE 2013;8(7):e67956.

\section{Kerkhoff 2014 \{published data only\}}

Kerkhoff AD, Wood R, Vogt M, Lawn SD. Predictive value of anemia for tuberculosis in HIV-infected patients in Sub-Saharan Africa: an indication for routine microbiological investigation using new rapid assays. Journal of Acquired Immune Deficiency Syndromes 2014;66(1):33-40.

\section{Khalil 2015 \{published data only\}}

Khalil KF, Butt T. Diagnostic yield of bronchoalveolar lavage gene Xpert in smear-negative and sputum-scarce pulmonary tuberculosis. Journal of the College of Physicians and Surgeons Pakistan 2015;25(2):115-8.

\section{Khan 2016 \{published data only\}}

Khan SU, Rahman H, Ayaz S, Qasim M, Jabbar A, Khurshid M, et al. GeneXpert assay for rapid detection of Mycobacterium tuberculosis complex in respiratory specimens from a high TB endemic area of Pakistan. Microbial Pathogenesis 2016;95:82-5.

Kim 2012 \{published data only\}

Kim SY, Kim H, Kim SY, Ra EK, Joo SI, Shin S, et al. The Xpert ${ }^{\circledR}$ MTB/RIF assay evaluation in South Korea, a country with an intermediate tuberculosis burden. International Journal of Tuberculosis and Lung Disease 2012;16(11):1471-6.

\section{Kim CH 2014 \{published data only\}}

Kim CH, Woo H, Hyun IG, Kim C, Choi JH, Jang SH, et al. A comparison between the efficiency of the Xpert MTB/RIF assay and nested PCR in identifying Mycobacterium tuberculosis during routine clinical practice. Journal of Thoracic Disease 2014;6(6):625-31.

Kim MJ 2015 \{published data only\}

Kim MJ, Nam YS, Cho SY, Park TS, Lee HJ. Comparison of the Xpert MTB/RIF Assay and real-time PCR for the detection of Mycobacterium tuberculosis. Annals of Clinical and Laboratory Science 2015;45(3):327-32.

Kim YW 2015 \{published data only\}

Kim YW, Seong MW, Kim TS, Yoo CG, Han SK, Yim JJ. Evaluation of Xpert ${ }^{\circledR}$ MTB/RIF assay: diagnosis and treatment outcomes in rifampicin-resistant tuberculosis. International Journal of Tuberculosis and Lung Disease 2015;19(10):1216-21.

\section{Lange 2017 \{published data only\}}

Lange B, Khan P, Kalmambetova G, Al-Darraji HA, Alland D, Antonenka $U$, et al. Diagnostic accuracy of the Xpert ${ }^{\circledR}$ MTB/ RIF cycle threshold level to predict smear positivity: a meta- 
analysis. International Journal of Tuberculosis and Lung Disease 2017;21(5):493-502.

\section{Laskar 2017 \{published data only\}}

Laskar N, Hossain MA, Nasreen SA, Kamal SM, Roy S, Nahar F, et al. Comparative yielding of BACTEC MGIT 960 and GeneXpert MTB/RIF assay for rapid diagnosis of drug resistance tuberculosis from sputum specimen. Mymensingh Medical Journal 2017;26(4):885-91.

\section{Lawn 2012a \{published data only\}}

Lawn SD, Kerkhoff AD, Vogt M, Ghebrekristos Y, Whitelaw A, Wood R. Characteristics and early outcomes of patients with Xpert MTB/RIF-negative pulmonary tuberculosis diagnosed during screening before antiretroviral therapy. Clinical Infectious Diseases 2012;54(8):1071-9.

\section{Lawn 2012b \{published data only\}}

Lawn SD, Kerkhoff AD, Vogt, M, Wood R. High diagnostic yield of tuberculosis from screening urine samples from HIV-infected patients with advanced immunodeficiency using the Xpert MTB/ RIF assay. Journal of Acquired Immune Deficiency Syndromes 2012;60(3):289-94

\section{Lawn 2012c \{published data only\}}

Lawn SD, Kerkhoff AD, Vogt M, Wood R. Clinical significance of lipoarabinomannan detection in urine using a low-cost pointof-care diagnostic assay for HIV-associated tuberculosis. AIDS 2012;26(13):1635-43.

\section{Lawn 2013 \{published data only\}}

Lawn SD, Kerkhoff AD, Vogt M, Wood R. HIV-associated tuberculosis: relationship between disease severity and the sensitivity of new sputum-based and urine-based diagnostic assays. BMC Medicine 2013;11:231.

\section{Lawn 2015 \{published data only\}}

Lawn SD, Kerkhoff AD, Burton R, Schutz C, Van Wyk G, Vogt M, et al. Rapid microbiological screening for tuberculosis in HIVpositive patients on the first day of acute hospital admission by systematic testing of urine samples using Xpert MTB/RIF: a prospective cohort in South Africa. BMC Medicine 2015;13:192.

\section{Lawn 2017 \{published data only\}}

Lawn SD, Kerkhoff AD, Burton R, Schutz C, Boulle A, Vogt M, et al. Diagnostic accuracy, incremental yield and prognostic value of Determine TB-LAM for routine diagnostic testing for tuberculosis in HIV-infected patients requiring acute hospital admission in South Africa: a prospective cohort. BMC Medicine 2017;15(1):67.

\section{Lebina 2016 \{published data only\}}

Lebina L, Fuller N, Osoba T, Scott L, Motlhaoleng K, Rakgokong M, et al. The use of Xpert MTB/RIFfor active case finding among TB contacts in North West Province, South Africa. Tuberculosis Research and Treatment 2016;2016:4282313.

\section{Lessells 2017 \{published data only\}}

Lessells RJ, Cooke GS, McGrath N, Nicol MP, Newell ML, Godfrey-Faussett P. Impact of point-of-care Xpert MTB/RIF on tuberculosis treatment initiation. A cluster-randomized trial.
American Journal of Respiratory and Critical Care Medicine 2017;196(7):901-10.

\section{Li 2016 \{published data only\}}

Li Q, Bao XD, Liu Y, Ou XC, Pang Y, Zhao YL. Comparison of two molecular assays for detecting smear negative pulmonary tuberculosis. Biomedical and Environmental Sciences 2016;29(4):248-53.

\section{Li 2017 \{published data only\}}

Li S, Liu B, Peng M, Chen M, Yin W, Tang H, et al. Diagnostic accuracy of Xpert MTB/RIF for tuberculosis detection in different regions with different endemic burden: a systematic review and meta-analysis. PLoS One 2017;12(7):e0180725.

Ligthelm 2011 \{published data only\}

Ligthelm LJ, Nicol MP, Hoek KG, Jacobson R, Van Helden PD, Marais BJ, et al. Xpert MTB/RIF for rapid diagnosis of tuberculous lymphadenitis from fine-needle-aspiration biopsy specimens. Journal of Clinical Microbiology 2011;49(11):3967-70.

\section{Lombardi 2017 \{published data only\}}

Lombardi G, Di Gregori V, Girometti N, Tadolini M, Bisognin F, Dal Monte P. Diagnosis of smear-negative tuberculosis is greatly improved by Xpert MTB/RIF. PLoS One 2017;12(4):e0176186.

\section{Mafort 2017 \{published data only\}}

Mafort TT, Rodrigues LS, Santos A, ReisL VT, Faria LF, Brito GMX, et al. Bronchoalveolar lavage GeneXpert MTB/RIF performance in smear-negative pulmonary tuberculosis-a tertiary care experience in Rio De Janeiro, Brazil. American Journal of Respiratory and Critical Care Medicine. American Thoracic Society International Conference 2017;195:A2085.

\section{Malbruny 2011 \{published data only\}}

Malbruny B, Le Marrec G, Courageux K, Leclercq R, Cattoir V. Rapid and efficient detection of Mycobacterium tuberculosis in respiratory and non-respiratory samples. International Journal of Tuberculosis and Lung Disease 2011;15(4):553-5.

Marlowe 2011 \{published data only\} Marlowe EM, Novak-Weekley SM, Cumpio J, Sharp SE, Momeny MA, Babst A, et al. Evaluation of the Cepheid Xpert MTB/RIF assay for direct detection of Mycobacterium tuberculosis complex in respiratory specimens. Journal of Clinical Microbiology 2011;49(4):1621-3.

Matabane 2015 \{published data only\}

Matabane MM, Ismail F, Strydom KA, Onwuegbuna O, Omar SV, Ismail N. Performance evaluation of three commercial molecular assays for the detection of Mycobacterium tuberculosis from clinical specimens in a high TB-HIV-burden setting. BMC Infectious Diseases 2015;15:508.

\section{Mave 2017 \{published data only\}}

Mave V, Nimkar S, Prasad H, Kadam D, Meshram S, Lokhande R, et al. Tuberculosis screening among persons with diabetes mellitus in Pune, India. BMC Infectious Diseases 2017;17(1):388. 
Maynard-Smith 2014 \{published data only\}

Maynard-Smith L, Larke N, Peters JA, Lawn SD. Diagnostic accuracy of the Xpert MTB/RIF assay for extrapulmonary and pulmonary tuberculosis when testing non-respiratory samples: a systematic review. BMC Infectious Diseases 2014;14:709.

Miller 2011 \{published data only\} Miller MB, Popowitch EB, Backlund MG, Ager EP. Performance of Xpert MTB/RIF RUO Assay and IS6110 Real-Time PCR for Mycobacterium tuberculosis detection in clinical samples. Journal of Clinical Microbiology 2011;49(10):3458-62.

Miotto 2012 \{published data only\}

Miotto P, Bigoni S, Migliori GB, Matteelli A, Cirillo DM. Early tuberculosis treatment monitoring by Xpert(R) MTB/RIF. European Respiratory Journal 2012; Vol. 39, issue 5:1269-71.

\section{Mntonintshi 2017 \{published data only\}}

Mntonintshi M, O'Mahony D, Mabunda S, Namugenyi KA. Undiagnosed tuberculosis in patients with HIV infection who present with severe anaemia at a district hospital. African Journal of Primary Health Care and Family Medicine 2017;9(1):e1-6.

\section{Modi 2016 \{published data only\}}

Modi S, Cavanaugh JS, Shiraishi RW, Alexander HL, McCarthy KD, Burmen B, et al. Performance of clinical screening algorithms for tuberculosis intensified case finding among people living with HIV in Western Kenya. PLoS One 2016; Vol. 11, issue 12:e0167685. [DOI: 10.1371/journal.pone.0167685]

\section{Mokaddas 2016 \{published data only\}}

Mokaddas EM, Saadaldeen H, Ahmad S. Comparison of two molecular methods and an automated liquid culture system for the early detection of Mycobacterium tuberculosis from both pulmonary and extrapulmonary specimens in Kuwait. International Journal of Mycobacteriology 2016;5 Suppl 1:S74-5.

\section{More 2017 \{published data only\}}

More SW, Parande MA, Kamble SW, Kamble MS. Profile of drugresistant tuberculosis in Western Maharashtra. Journal of Family Medicine and Primary Care 2017;6(1):29-33.

\section{Morozova 2016 \{published data only\}}

Morozova TI, Salina T. The results of drug susceptibility testing of Mycobacterium tuberculosis to rifampicin by Xpert MTB/RIF BACTEC MGIT 960 as compared with the method of seeding on solid nutrient media. European Respiratory Journal. European Respiratory Society Annual Congress 2016;48(Suppl 60):PA2783.

\section{Moure 2012 \{published data only\}}

Moure R, Martin R, Alcaide F. Effectiveness of an integrated real-time PCR method for detection of the Mycobacterium tuberculosis complex in smear-negative extrapulmonary samples in an area of low tuberculosis prevalence. Journal of Clinical Microbiology 2012;50(2):513-5.

\section{Mukherjee 2017 \{published data only\}}

Mukherjee S, Biswas D, Begum S, Ghosh P, Paul A, Sarkar S. Evaluation of cartridge based nucleic acid amplification test in diagnosis of pulmonary tuberculosis. Journal of Evolution of Medical and Dental Sciences-JEMDS 2017;6(74):5281-6.

Mulder 2017 \{published data only\}

Mulder C, Mgode GF, Ellis H, Valverde E, Beyene N, Cox C, et al. Accuracy of giant African pouched rats for diagnosing tuberculosis: comparison with culture and Xpert ${ }^{\circledast}$ MTB/ RIF. International Journal of Tuberculosis and Lung Disease 2017;21(11):1127-33.

Muñoz 2013 \{published data only\}

Muñoz L, Moure R, Porta N, Gonzalez L, Guerra R, Alcaide F, et al. GeneXpert ${ }^{\circledast}$ for smear-negative pulmonary tuberculosis: does it play a role in low-burden countries?. Diagnostic Microbiology Infectious Disease 2013;75(3):325-6.

Myneedu 2014 \{published data only\}

Myneedu VP, Behera D, Verma AK, Bhalla M, Singh N, Arora J, et al. Xpert ${ }^{\circledR}$ MTB/RIF assay for tuberculosis diagnosis: evaluation in an Indian setting. International Journal of Tuberculosis and Lung Disease 2014;18(8):958-60.

Naidoo 2016 \{published data only\}

Naidoo P, Dunbar R, Lombard C, Du Toit E, Caldwell J, Detjen A, et al. Comparing tuberculosis diagnostic yield in smear/culture and Xpert1 MTB/RIF-based algorithms using a non-randomised stepped-wedge design. PLoS One 2016;11(3):e0150487.

\section{Narasimooloo 2012 \{published data only\}}

Narasimooloo R, Ross A. Delay in commencing treatment for MDR TB at a specialised TB treatment centre in KwaZulu-Natal. South African Medical Journal 2012;102(6 Pt 2):360-2.

\section{Ng 2018 \{published data only\}}

Ng KC, Van Deun A, Meehan CJ, Torrea G, Driesen M, Gabriels S, et al. Xpert Ultra can unambiguously identify specific rifampin resistance-conferring mutations. Journal of Clinical Microbiology 2018;56(9):e00686-18. [DOI: 10.1128/JCM.00686-18]

\section{Nguyen 2018 \{published data only\}}

Nguyen VA, Nguyen HV, Dinh TV, Du HH, Do CN, Marks GB, et al. Evaluation of Loopamp ${ }^{\mathrm{TM}}$ MTBC detection kit for diagnosis of pulmonary tuberculosis at a peripheral laboratory in a high burden setting. Diagnostic Microbiology and Infectious Disease 2017;90(3):190-5.

\section{Ngwira 2017 \{published data only\}}

Ngwira LG, Khundi M, Barnes GL, Nkhoma A, Murowa M, Cohn S, et al. Screening for tuberculosis with Xpert MTB/RIF versus fluorescent microscopy among people newly diagnosed with HIV in rural Malawi: a cluster-randomized trial. Journal of the International AIDS Society 2017;20:93-4.

\section{Nhu 2013 \{published data only\}}

Nhu NT, Ha DT, Anh ND, Thu DD, Duong TN, Quang ND, et al. Evaluation of Xpert MTB/RIF and MODS assay for the diagnosis of pediatric tuberculosis. BMC Infectious Diseases 2013;13:31

\section{Nicol 2011 \{published data only\}}

Nicol MP, Workman L, Isaacs W, Munro J, Black F, Eley B, et al. Accuracy of the Xpert MTB/RIF test for the diagnosis of 
pulmonary tuberculosis in children admitted to hospital in Cape Town, South Africa: a descriptive study. Lancet Infectious Diseases 2011;11(11):819-24.

\section{Ninan 2016 \{published data only\}}

Ninan MM, Gowri M, Christopher DJ, Rupali P, Michael JS. The diagnostic utility of line probe assays for multidrug-resistant tuberculosis. Pathogens and Global Health 2016;110(4-5):194-9.

\section{Nosova 2013b $\{$ published data only\}}

Nosova EY, Krasnova MA, Galkina KY, Makarova MV, Litvinov VI, Moroz AM. Comparing performance of "TB-BIOCHIP", "Xpert MTB/RIF" and "genotype MTBDRplus" assays for fast identification of mutations in the Mycobacterium tuberculosis complex in sputum from TB patients. Molekuliarnaia Biologiia (Mosk) 2013;47(2):267-74.

\section{Ntinginya 2012 \{published data only\}}

Ntinginya EN, Squire SB, Millington KA, Mtafya B, Saathoff E, Heinrich N, et al. Performance of the Xpert ${ }^{\circledR}$ MTB/RIF assay in an active case-finding strategy: a pilot study from Tanzania. International Journal of Tuberculosis and Lung Disease 2012;16(11):1468-70.

\section{O'Grady 2012 \{published data only\}}

O'Grady J, Bates M, Chilukutu L, Mzyece J, Cheelo B, Chilufya M, et al. Evaluation of the Xpert MTB/RIF assay at a tertiary care referral hospital in a setting where tuberculosis and $\mathrm{HIV}$ infection are highly endemic. Clinical Infectious Diseases 2012;55(9):1171-8.

\section{Omrani 2014 \{published data only\}}

Omrani AS, Al-Otaibi MF, Al-Ateah SM, Al-Onazi FM, Baig K, ElKhizzi NA, et al. GeneXpert MTB/RIF testing in the management of patients with active tuberculosis; a real life experience from Saudi Arabia. Infection and Chemotherapy 2014;46(1):30-4.

\section{Opota 2016 \{published data only\}}

Opota O, Senn L, Prod'hom G, Mazza-Stalder J, Tissot F, Greub G, et al. Added value of molecular assay Xpert MTB/RIF compared to sputum smear microscopy to assess the risk of tuberculosis transmission in a low-prevalence country. Clinical Microbiology and Infection 2016;22(7):613-9.

\section{Osman 2014 \{published data only\}}

Osman M, Simpson JA, Caldwell J, Bosman M, Nicol MP. GeneXpert MTB/RIF version G4 for identification of rifampinresistant tuberculosis in a programmatic setting. Journal of Clinical Microbiology 2014;52(2):635-7.

\section{Ou 2015 \{published data only\}}

Ou X, Xia H, Li Q, Pang Y, Wang S, Zhao B, et al. A feasibility study of the Xpert MTB/RIF test at the peripheral level laboratory in China. International Journal of Infectious Diseases 2015;31:41-6.

\section{Ozkutuk 2014 \{published data only\}}

Ozkutuk N, Surucüoglu S. Evaluation of the Xpert MTB/RIF assay for the diagnosis of pulmonary and extrapulmonary tuberculosis in an intermediate-prevalence setting. Mikrobiyoloji Bulteni 2014;48(2):223-32.

\section{Pandey P 2017 \{published data only\}}

Pandey P, Pant ND, Rijal KR, Shrestha B, Kattel S, Banjara MR, et al. Diagnostic accuracy of GeneXpert MTB/RIF assay in comparison to conventional drug susceptibility testing method for the diagnosis of multidrug-resistant tuberculosis. PLoS One 2017;12(1):e0169798.

\section{Pandey S 2017 \{published data only\}}

Pandey S, Congdon J, McInnes B, Pop A, Coulter C. Evaluation of the GeneXpert MTB/RIF assay on extrapulmonary and respiratory samples other than sputum: a low burden country experience. Pathology 2017;49(1):70-4.

\section{Parcell 2017 \{published data only\}}

Parcell BJ, Jarchow-MacDonald AA, Seagar AL, Laurenson IF, Prescott GJ, Lockhart M. Three year evaluation of Xpert MTB/RIF in a low prevalence tuberculosis setting: A Scottish perspective. Journal of Infection 2017;74(5):466-72.

\section{Patil 2014 \{published data only\}}

Patil N, Saba H, Marco A, Samant R, Mukasa L. Initial experience with GeneXpert MTB/RIF assay in the Arkansas Tuberculosis Control Program. Australasian Medical Journal 2014;7(5):203-7.

\section{Patil 2017 \{published data only\}}

Patil S, Narwade S, Mirza M. Bronchial wash Gene Xpert MTB/RIF in lower lung field tuberculosis: sensitive, superior, and rapid in comparison with conventional diagnostic techniques. Journal of Translational Internal Medicine 2017;5(3):174-81.

\section{Peter 2012 \{published data only\}}

Peter JG, Theron G, Muchinga TE, Govender U, Dheda K. The diagnostic accuracy of urine-based Xpert MTB/RIF in HIVinfected hospitalized patients who are smear-negative or sputum scarce. PLoS One 2012;7(7):e39966.

\section{Peter 2013 \{published data only\}}

Peter JG, Theron G, Pooran A, Thomas J, Pascoe M, Dheda K. Comparison of two methods for acquisition of sputum samples for diagnosis of suspected tuberculosis in smear-negative or sputum-scarce people: a randomised controlled trial. Lancet Respiratory Medicine 2013;1(6):471-8.

\section{Peter 2015 \{published data only\}}

Peter J, Theron G, Chanda D, Clowes P, Rachow A, Lesosky M, et al. Test characteristics and potential impact of the urine LAM lateral flow assay in HIV-infected outpatients under investigation for TB and able to self-expectorate sputum for diagnostic testing. BMC Infectious Diseases 2015;15:262.

\section{Rachow 2012 \{published data only\}}

Rachow A, Clowes P, Saathoff E, Mtafya B, Michael E, Ntinginya $E N$, et al. Increased and expedited case detection by Xpert MTB/RIF assay in childhood tuberculosis: a prospective cohort study. Clinical Infectious Diseases 2012;54(10):1388-96.

\section{Rahman 2016 \{published data only\}}

Rahman A, Sahrin M, Afrin S, Earley K, Ahmed S, Rahman SM, et al. Comparison of Xpert MTB/RIF assay and GenoType MTBDRplus DNA Pprobes for detection of mutations associated 
with rifampicin resistance in Mycobacterium tuberculosis. PLOS One 2016;11(4):e0152694.

\section{Raizada 2015 \{published data only\}}

Raizada N, Sachdeva KS, Sreenivas A, Kulsange S, Gupta RS, Thakur R, et al. Catching the missing million: experiences in enhancing TB \& DR-TB detection by providing upfront Xpert MTB/RIF testing for people living with HIV in India. PLoS One 2015;10(2):e0116721.

\section{Ramamurthy 2016 \{published data only\}}

Ramamurthy K, Bhat S, Shenoy S, Rangnekar A. Xpert Mycobacterium tuberculosis/rifampicin assay: a boon in tuberculosis diagnostics. Asian Journal of Pharmaceutical and Clinical Research 2016;9(5):225-7.

\section{Ramirez 2014 \{published data only\}}

Ramirez HL, Garcia-Clemente MM, Alvarez-Alvarez C, PalacioGutierrez JJ, Pando-Sandoval A, Gagatek S, et al. Impact of the Xpert ${ }^{\circledR}$ MTB/RIF molecular test on the late diagnosis of pulmonary tuberculosis. International Journal of Tuberculosis and Lung Disease 2014;18(4):435-7.

\section{Reechaipichitkul 2016 \{published data only\}}

Reechaipichitkul W, Phetsuriyawong A, Chaimanee P, Ananta P. Diagnostic test of sputum GeneXpert MTB/RIF for smear negative pulmonary tuberculosis. Southeast Asian Journal of Tropical Medicine and Public Health 2016;47(3):457-66.

\section{Reed 2016 \{published data only\}}

Reed JL, Walker ZJ, Basu D, Allen V, Nicol MP, Kelso DM, et al. Highly sensitive sequence specific qPCR detection of Mycobacterium tuberculosis complex in respiratory specimens. Tuberculosis (Edinb) 2016;101:114-24.

\section{Rees 2018 \{published data only\}}

Rees K, Muditambi N, Maswanganyi M, Railton J, Mclntyre JA, Struthers HE, et al. The impact of implementing a Xpert MTB/ RIF algorithm on drug-sensitive pulmonary tuberculosis: a retrospective analysis. Epidemiology \& Infection 2018;146:246255.

\section{Rossato 2018 \{published data only\}}

Rossato Silva D, Sotgiu G, D'Ambrosio L, Rodrigues Pereira G, Silva Barbosa M, Dutra Dias NJ, et al. Diagnostic performances of the Xpert MTB/RIF in Brazil. Respiratory Medicine 2018;134:12-5.

\section{Rufai 2014 \{published data only\}}

Rufai SB, Kumar P, Singh A, Prajapati S, Balooni V, Singh S. Comparison of Xpert MTB/RIF with line probe assay for detection of rifampin-monoresistant Mycobacterium tuberculosis. Journal of Clinical Microbiology 2014; Vol. 52, issue 6:1846-52.

\section{Ruiz 2017 \{published data only\}}

Ruiz P, Causse M, Vaquero M, Gutierrez JB, Casal M. Evaluation of a new automated Abbott RealTime MTB RIF/INH assay for qualitative detection of rifampicin/isoniazid resistance in pulmonary and extra-pulmonary clinical samples of
Mycobacterium tuberculosis. Infection and Drug Resistance 2017;10:463-7.

\section{Sachdeva 2015 \{published data only\}}

Sachdeva KS, Raizada N, Sreenivas A, Van't Hoog AH, Van den Hof S, Dewan PK, et al. Use of Xpert MTB/RIF in decentralized public health settings and Its effect on pulmonary TB and DR-TB case finding in India. PLoS One 2015;10(5):e0126065.

\section{Saeed 2017 \{published data only\}}

Saeed M, Iram S, Hussain S, Ahmed A, Akbar M, Aslam M. GeneXpert: A new tool for the rapid detection of rifampicin resistance in mycobacterium tuberculosis. Journal of the Pakistan Medical Association 2017;67(2):270-4.

\section{Sanchez-Padilla 2015 \{published data only\}}

Sanchez-Padilla E, Merker M, Beckert P, Jochims F, Dlamini T, Kahn P, et al. Detection of drug-resistant tuberculosis by Xpert MTB/RIF in Swaziland. New England Journal of Medicine 2015;372(12):1181-2.

\section{Sauzullo 2016 \{published data only\}}

Sauzullo I, Rodio DM, Facchinetti S, Puggioni G, De Angelis M, Goldoni P, et al. Diagnostic accuracy of Xpert MTB/RIF versus smear microscopy in the early diagnosis tuberculosis in the real life of "Umberto I" Hospital Rome. New Microbiologica 2016;39(4):304-6.

\section{Shah 2014 \{published data only\}}

Shah M, Ssengooba W, Armstrong D, Nakiyingi L, Holshouser M, Ellner JJ, et al. Comparative performance of urinary lipoarabinomannan assays and Xpert MTB/RIF in HIV-infected individuals. AIDS 2014;28(9):1307-14.

\section{Shenai 2013 \{published data only\}}

Shenai S, Amisano D, Ronacher K, Kriel M, Banada PP, Song T, et al. Exploring alternative biomaterials for diagnosis of pulmonary tuberculosis in HIV-negative patients by use of the GeneXpert MTB/RIF assay. Journal of Clinical Microbiology 2013;51(12):4161-6.

\section{Shilpa 2017 \{published data only\}}

Shilpa, Nadagir SD, Jnaneshwara KB, Patil AB, Pendari AG, Chikkaraddi U. Detection of rifampicin resistance in HIV seropositive individuals with suspected pulmonary tuberculosis by using CBNAAT. Journal of Pure and Applied Microbiology 2017;11(1):387-92.

\section{Smith 2014 \{published data only\}}

Smith P, Van Esch A, Wallace M, Wood R, Bekker LG. GeneXpert TB 8: A point-of-care diagnostic pilot. South African Medical Journal 2014;104(8):524.

\section{Somashekar 2014 \{published data only\}}

Somashekar N, Chadha VK, Praseeja P, Sharada MA, Chandrakala GR, Srivastava R, et al. Role of pre-Xpert(R) screening using chest $X$-ray in early diagnosis of smear-negative pulmonary tuberculosis. International Journal of Tuberculosis and Lung Disease 2014;18(10):1243-4. 
Somily 2016 \{published data only\}

Somily AM, Barry MA, Habib HA, Alotaibi FE, Al-Zamil FA, Khan MA, et al. Evaluation of GeneXpert MTB/RIF for detection of Mycobacterium tuberculosis complex and rpo $B$ gene in respiratory and non-respiratory clinical specimens at a tertiary care teaching hospital in Saudi Arabia. Saudi Medical Journal 2016;37(12):1404-7.

\section{Strydom 2015 \{published data only\}}

Strydom K, Ismail F, Matabane MM, Onwuegbuna O, Omar SV, Ismail N. Comparison of three commercial molecular assays for detection of rifampin and isoniazid resistance among Mycobacterium tuberculosis isolates in a high-HIV-prevalence setting. Journal of Clinical Microbiology 2015;53(9):3032-4.

\section{Sureshbabu 2016 \{published data only\}}

Sureshbabu R, Lakshmi Murali A, Palaniswamy M. Molecular diagnosis of drug resistance tuberculosis In the districts of Tamilnadu. International Journal of Pharma and Bio Sciences 2016;7(4):B42-6.

\section{Tadesse 2016b \{published data only\}}

Tadesse M, Aragaw D, Dimah B, Efa F, Abebe G. Xpert MTB/ $\mathrm{RIF}$ for rapid detection of rifampicin-resistant Mycobacterium tuberculosis from pulmonary tuberculosis patients in southwest Ethiopia. International Journal of Mycobacteriology 2016;5 Suppl 1:S48-9.

\section{Tahseen 2016 \{published data only\}}

Tahseen S, Qadeer E, Khanzada FM, Rizvi AH, Dean A, Van Deun A, et al. Use of Xpert ${ }^{\circledR}$ MTB/RIF assay in the first national anti-tuberculosis drug resistance survey in Pakistan. International Journal Tuberculoisis Lung Disease 2016;20(4):448-55

\section{Tan 2017 \{published data only\}}

Tan Y, Li Q, Wang Q, Sun H, Chen J, Cai X, et al. Evaluation of the MTBDRplus 2.0 assay for the detection of multidrug resistance among persons with presumptive pulmonary TB in China. Science Reports 2017;7(1):3364.

\section{Taylor 2012 \{published data only\}}

Taylor N, Gaur RL, Baron EJ, Banaei N. Can a simple flotation method lower the limit of detection of Mycobacterium tuberculosis in extrapulmonary samples analyzed by the GeneXpert MTB/RIF assay?. Journal of Clinical Microbiology 2012;50(7):2272-6.

\section{Teo 2011 \{published data only\}}

Teo J, Jureen R, Chiang D, Chan D, Lin R. Comparison of two nucleic acid amplification assays, the Xpert MTB/RIF and the amplified Mycobacterium tuberculosis Direct assay, for the detection of Mycobacterium tuberculosis in respiratory and non-respiratory specimens. Journal of Clinical Microbiology 2011;49(10):3659-62.

\section{Theron 2012 \{published data only\}}

Theron G, Peter J, Lenders L, Van Zyl-Smit R, Meldau R, Govender U, et al. Correlation of mycobacterium tuberculosis specific and non-specific quantitative Th1 T-cell responses with bacillary load in a high burden setting. PLoS One 2012; 7(5):e37436.

\section{Theron 2014b \{published data only\}}

Theron G, Peter J, Calligaro G, Meldau R, Hanrahan C, Khalfey H, et al. Determinants of PCR performance (Xpert MTB/RIF), including bacterial load and inhibition, for TB diagnosis using specimens from different body compartments. Science Reports 2014;4:5658.

\section{Theron 2016 \{published data only\}}

Theron G, Venter R, Calligaro G, Smith L, Limberis J, Meldau R, et al. Xpert MTB/RIF results in patients with previous tuberculosis: can we distinguish true from false positive results?. Clinical Infectious Diseases 2016;62(8):995-1001.

\section{Theron 2018 \{published data only\}}

Theron G, Venter R, Smith L, Esmail A, Randall P, Sood V, et al. False positive Xpert MTB/RIF results in re-tested patients with previous tuberculosis: frequency, profile, and prospective clinical outcomes. Journal of Clinical Microbiology 2018;56(3):pii: e01696-17.

\section{Thibbadee 2016 \{published data only\}}

Thibbadee C. Evaluation of decentralised use of the Xpert MTB/ RRIF test for diagnosis of tuberculosis and multidrug resistance in Rayong hospital, Thailand. Respirology 2016;21 (Supplement 3):198.

\section{Thit 2017 \{published data only\}}

Thit SS, Aung NM, Htet ZW, Boyd MA, Saw HA, Anstey NM, et al. The clinical utility of the urine-based lateral flow lipoarabinomannan assay in HIV-infected adults in Myanmar: an observational study. BMC Medicine 2017;15(1):145.

\section{To 2017 \{published data only\}}

To KW, Kam KM, Lee SS, Chan KP, Yip T, Lo R, et al. Clinical application of GeneXpert on BAL samples in management of TB in intermediate burden area. Chest 2017;152 (4 Supplement 1):A194.

\section{Tortoli 2012 \{published data only\}}

Tortoli E, Russo C, Piersimoni C, Mazzola E, Dal Monte P, Pascarella M, et al. Clinical validation of Xpert MTB/RIF for the diagnosis of extrapulmonary tuberculosis. European Respiratory Journal 2012;40(2):442-7.

\section{Ullah 2016 \{published data only\}}

Ullah I, Shah AA, Basit A, Ali M, Khan A, Ullah U, et al. Rifampicin resistance mutations in the $81 \mathrm{bp}$ RRDR of rpoB gene in Mycobacterium tuberculosis clinical isolates using Xpert MTB/ RIF in Khyber Pakhtunkhwa, Pakistan: a retrospective study. BMC Infectious Diseases 2016;16:413.

\section{Ullah 2017 \{published data only\}}

Ullah I, Javaid A, Masud H, Ali M, Basit A, Ahmad W, et al. Rapid detection of Mycobacterium tuberculosis and rifampicin resistance in extrapulmonary tuberculosis and sputum smearnegative pulmonary suspects using Xpert MTB/RIF. Journal of Medical Microbiology 2017;66(4):412-8. 
Vadwai 2011 \{published data only\}

Vadwai V, Boehme C, Nabeta P, Shetty A, Alland D, Rodrigues C. Xpert MTB/RIF: a new pillar in diagnosis of extrapulmonary tuberculosis?. Journal of Clinical Microbiology 2011;49(7):2540-5.

\section{Van Kampen 2015 \{published data only\}}

Van Kampen SC, Tursynbayeva A, Koptleuova A, Murzakhmetova Z, Bigalieva L, Aubakirova M, et al. Effect of introducing Xpert MTB/RIF to test and treat individuals at risk of multidrug-resistant tuberculosis in Kazakhstan: a prospective cohort study. PLoS One 2015;10(7):e0132514.

\section{Van Rie 2011 \{published data only\}}

Van Rie A. A single Xpert MTB/RIF test of sputum for diagnosis of tuberculosis and multidrug resistance shows high sensitivity and specificity and reduces diagnosis and treatment delays. Evidence Based Medicine 2011;16(6):174-5.

\section{Walters 2012 \{published data only\}}

Walters E, Gie RP, Hesseling AC, Friedrich SO, Diacon AH. Rapid diagnosis of pediatric intrathoracic tuberculosis from stool samples using the Xpert MTB/RIF Assay: a pilot study. Pediatric Infectious Disease Journal 2012;31(12):1316.

\section{Walusimbi 2013b $\{$ published data only\}}

Walusimbi S, Bwanga F, De Costa A, Haile M, Joloba M, Hoffner S. Meta-analysis to compare the accuracy of GeneXpert, MODS and the WHO 2007 algorithm for diagnosis of smearnegative pulmonary tuberculosis. BMC Infectious Diseases 2013;13:507.

\section{Wang 2015 \{published data only\}}

Wang XW, Pappoe F, Huang Y, Cheng XW, Xu DF, Wang H, et al. Xpert MTB/RIF assay for pulmonary tuberculosis and rifampicin resistance in children: a meta-analysis. Clinical Laboratory 2015;61(11):1775-85.

\section{Wang 2016 \{published data only\}}

Wang SF, Ou XC, Li Q, Zheng HW, Wang YF, Zhao YL. The Abbott RealTime MTB assay and the Cepheid GeneXpert assay show comparable performance for the detection of Mycobacterium tuberculosis in sputum specimens. International Journal of Infectious Diseases 2016;45:78-80.

\section{Williamson 2012a $\{$ published data only\}}

Williamson DA, Roberts SA, Bower JE, Vaughan R, Newton S, Lowe $\mathrm{O}$, et al. Clinical failures associated with rpoB mutations in phenotypically occult multidrug-resistant Mycobacterium tuberculosis. International Journal of Tuberculosis and Lung Disease 2012;16(2):216-20.

\section{Wood 2012 \{published data only\}}

Wood R, Racow K, Bekker LG, Middelkoop K, Vogt M, Kreiswirth BN, et al. Lipoarabinomannan in urine during tuberculosis treatment: association with host and pathogen factors and mycobacteriuria. BMC Infectious Diseases 2012;12:47.

\section{Xie 2017 \{published data only\}}

Xie YL, Chakravorty S, Armstrong DT, Hall SL, Via LE, Song T, et al. Evaluation of a rapid molecular drug-susceptibility test for tuberculosis. New England Journal of Medicine 2017;377(11):1043-54

\section{Yadav 2017 \{published data only\}}

Yadav R, Sharma N, Khaneja R, Agarwal P, Kanga A, Behera D, et al. Evaluation of the TB-LAMP assay for the rapid diagnosis of pulmonary tuberculosis in northern India. International Journal of Tuberculosis and Lung Disease 2017;21(10):1150-3.

Yan 2016 \{published data only\} Yan L, Xiao H, Zhang Q. Systematic review: Comparison of Xpert MTB/RIF, LAMP and SAT methods for the diagnosis of pulmonary tuberculosis. Tuberculosis (Edinb) 2016;96:75-86.

\section{Zar 2012 \{published data only\}}

Zar HJ, Workman L, Isaacs W, Munro J, Black F, Eley B, et al. Rapid molecular diagnosis of pulmonary tuberculosis in children using nasopharyngeal specimens. Clinical Infectious Diseases 2012;55(8):1088-95.

\section{Zemlyansky 2016 \{published data only\}}

Zemlyansky OA, Tyurina EB, Bashkirev AA, Kalyuzhnaya EV, Zemlyanskaya LO. Experience and efficiency of laboratory diagnosis of tuberculosis with PCR detector system GeneXpert in Belgorod region. International Journal of Pharmacy and Technology 2016;8(4):27072-9.

\section{References to ongoing studies}

Koenig 2018 \{published data only\}

A trial of same-day testing and treatment to improve outcomes among symptomatic patients newly diagnosed with HIV. Ongoing study 16 May 2017

\section{Reid 2018 \{published data only\}}

Achieving tuberculosis control In Zambia. Ongoing study 13 April 2018.

\section{Theron 2018a $\{$ published data only\}}

Improving tuberculosis diagnosis and treatment through Basic, Applied and health systems Research (BAR). Ongoing study 29 November 2017

Theron 2018b \{published data only\}

Xpert Ultra and Xpert HIV-VL in people living with HIV (UltraHIV). Ongoing study 15 June 2017.

\section{Zhang 2018 \{published data only\}}

Diagnostic accuracy of Xpert MTB/RIF Ultra for tuberculous bronchoalveolar lavage fluid in HIV-infected adults: a prospective cohort study. Ongoing study 12 February 2018.

\section{Additional references}

\section{American Thoracic Society 2000}

American Thoracic Society, the Centers for Disease Control and Prevention, Infectious Disease Society of America. Diagnostic 
Standards and Classification of Tuberculosis in Adults and Children. This official statement of the American Thoracic Society and the Centers for Disease Control and Prevention was adopted by the ATS Board of Directors, July 1999. This statement was endorsed by the Council of the Infectious Disease Society of America, September 1999. American Journal Respiratory and Critical Care Medicine 2000;161(4 Pt 1):1376-95.

\section{Auld 2016a}

Auld AF, Fielding KL, Gupta-Wright A, Lawn SD. Xpert MTB/RIF why the lack of morbidity and mortality impact in intervention trials?. Transactions of the Royal Society of Tropical Medicine and Hygiene 2016;110(8):432-44.

\section{Balshem 2011}

Balshem H, Helfand M, Schünemann HJ, Oxman AD, Kunz R, Brozek J, et al. GRADE guidelines: 3. Rating the quality of evidence. Journal of Clinical Epidemiology 2011;64(4):401-6.

\section{Banada 2010}

Banada PP, Sivasubramani SK, Blakemore R, Boehme C, Perkins MD, Fennelly K, et al. Containment of bioaerosol infection risk by the Xpert MTB/RIF assay and its applicability to point-of-care settings. Journal of Clinical Microbiology 2010;48(10):3551-7.

\section{Berhanu 2018}

Berhanu RH, David A, Da Silva P, Shearer K, Sanne I, Stevens W, et al. Performance of Xpert MTB/RIF, Xpert Ultra, and Abbott RealTime MTB for diagnosis of pulmonary tuberculosis in a high-HIV-burden setting. Journal of Clinical Microbiology 2018;56(12):e00560-18.

\section{Beynon 2018}

Beynon F, Theron G, Respeito D, Mambuque E, Saavedra B, Bulo $\mathrm{H}$, et al. Correlation of Xpert MTB/RIF with measures to assess Mycobacterium tuberculosis bacillary burden in high HIV burden areas of Southern Africa. Scientific Reports 2018;8(1):5201.

\section{Blakemore 2010}

Blakemore R, Story E, Helb D, Kop J, Banada P, Owens MR, et al. Evaluation of the analytical performance of the Xpert MTB/RIF assay. Journal of Clinical Microbiology 2010;48(7):2495-501.

\section{Bossuyt 2008}

Bossuyt PM, Leeflang MM. Chapter 6: Developing criteria for including studies. In: Cochrane Handbook for Systematic Reviews of Diagnstic Test Accuracy, Version 0.4 [updated September 2008]. The Cochrane Collaboration, 2008. Available at methods.cochrane.org/sites/methods.cochrane.org.sdt/ files/public/uploads/Chapter06-Including-Studies \%20\%28September-2008\%29.pdf.

\section{Bossuyt 2015}

Bossuyt PM, Reitsma JB, Bruns DE, Gatsonis CA, Glasziou PP, Irwig L, et al. STARD 2015: an updated list of essential items for reporting diagnostic accuracy studies. BMJ 2015;351:h5527. [DOI: 10.1136/bmj.h5527]

\section{Boyles 2014}

Boyles TH, Hughes J, Cox V, Burton R, Meintjes G, Mendelson M. False-positive Xpert ${ }^{\circledR} \mathrm{MTB} / \mathrm{RIF}$ assays in previously treated patients: need for caution in interpreting results. The International Journal of Tuberculosis and Lung Disease 2014;18(7):876-8.

\section{Boyles 2017}

Boyles TH. Why do clinical trials of Xpert ${ }^{\circledR}$ MTB/RIF fail to show an effect on patient relevant outcomes?. International Journal of Tuberculosis and Lung Disease 2017;21(3):249-50.

\section{Broger 2018}

Broger T, Sosse B, Du Toit E, Kerkhoff AD, Schutz C, Reipold El, et al. Novel high sensitivity tuberculosis point-of-care test for people living with HIV. Preprints with The Lancet. ssrn.com/ abstract=3254479 2018 .

\section{Buzoianu 2008}

Buzoianu M, Kadane JB. Adjusting for verification bias in diagnostic test evaluation: a Bayesian approach. Statististics in Medicine 2008;27(13):2453-73.

\section{Cazabon 2018}

Cazabon D, Pande T, Kik S, Van Gemert W, Sohn H, Denkinger C, et al. Market penetration of Xpert MTB/RIF in high tuberculosis burden countries: A trend analysis from 2014 - 2016 [version 2; referees: 4 approved]. Gates Open Research 2018;2:35. [DOI: 10.12688/gatesopenres.12842.1]

\section{Cepheid 2009}

Cepheid. Brochure: Xpert ${ }^{\circledR}$ MTB/RIF. Two-hour detection of MTB and resistance to rifampicin. cepheid.com/ administrator/components/com_productcatalog/ library-files/8dbd1dd8b83a9780dd88a0d9852ffd98a464e0bea6122c5c648ccdf617ecbd0c-Xpert-MTBRIF-BrochureEU-0089-02-LOR.pdf. Sunnyvale, (Accessed 16 May 2019).

\section{Chang 2012}

Chang K, Lu W, Wang J, Zhang K, Jia S, Li F, et al. Rapid and effective diagnosis of tuberculosis and rifampicin resistance with Xpert MTB/RIF assay: a meta-analysis. Journal of Infection 2012;64(6):580-8.

\section{Chu 2006}

Chu H, Cole SR. Bivariate meta-analysis of sensitivity and specificity with sparse data: a generalized linear mixed model approach. Journal of Clinical Epidemiology 2006;59(12):1331-2.

\section{Chu 2009}

Chu H, Chen S, Louis TA. Random effects models in a metaanalysis of the accuracy of two diagnostic tests without a gold standard. Journal of the American Statistical Association 2009;104(486):512-23.

\section{Churchyard 2015}

Churchyard GJ, Stevens WS, Mametja LD, McCarthy KM, Chihota V, Nicol MP, et al. Xpert MTB/RIF versus sputum microscopy as the initial diagnostic test for tuberculosis: a cluster-randomised trial embedded in South African roll-out of Xpert MTB/RIF. Lancet Global Health 2015;3(8):e450-7. 


\section{Covidence 2017 [Computer program]}

Veritas Health Innovation. Covidence systematic review software. Available at www.covidence.org. Melbourne: Veritas Health Innovation, 2017.

\section{Cox 2014}

Cox HS, Mbhele S, Mohess N, Whitelaw A, Muller O, Zemanay W, et al. Impact of Xpert MTB/RIF for TB diagnosis in a primary care clinic with high TB and HIV prevalence in South Africa: a pragmatic randomised trial. PLoS Medicine 2014;11(11):e1001760.

\section{Flores 2005}

Flores LL, Pai M, Colford JM Jr, Riley LW. In-house nucleic acid amplification tests for the detection of Mycobacterium tuberculosis in sputum specimens: meta-analysis and metaregression. BMC Microbiology 2005;5:55.

\section{Friedrich 2013}

Friedrich SO, Rachow A, Saathoff E, Singh K, Mangu CD, Dawson R, et al. Pan African Consortium for the Evaluation of Anti-tuberculosis Antibiotics (PanACEA). Assessment of the sensitivity and specificity of Xpert MTB/RIF assay as an early sputum biomarker of response to tuberculosis treatment. Lancet Respiratory Medicine 2013;1(6):462-70.

\section{Getahun 2007}

Getahun H, Harrington M, O'Brien R, Nunn P. Diagnosis of smear-negative pulmonary tuberculosis in people with HIV infection or AIDS in resource-constrained settings: informing urgent policy changes. Lancet 2007;369(9578):2042-9.

\section{Getahun 2010}

Getahun H, Gunneberg C, Granich R, Nunn P. HIV infectionassociated tuberculosis: the epidemiology and the response. Clinical Infectious Diseases 2010;50(Suppl 3):201-7.

\section{GLI 2015}

Global Laboratory Initiative. Guide for providing technical support to TB laboratories in low- and middle-income countries. 2015. stoptb.org/wg/gli/assets/documents/ guideforprovidingtechnicalsupport_gb_web.pdf (accessed 23 May 2019).

\section{GLI 2018}

Global Laboratory Initiative. GLI model TB diagnostic algorithms, 2018. www.stoptb.org/wg/gli/gat.asp (accessed 11 August 2018).

\section{Gopinath 2010}

Gopinath K, Singh S. Non-tuberculous mycobacteria in TBendemic countries: Are we neglecting the danger?. PLOS Neglected Tropical Diseases 2010;4(4):e615.

\section{GRADEpro GDT 2015 [Computer program]}

McMaster University (developed by Evidence Prime). GRADEpro GDT. Version accessed 29 October 2016. Hamilton (ON): McMaster University (developed by Evidence Prime), 2015.

\section{Gupta-Wright 2018}

Gupta-Wright A, Corbett EL, Van Oosterhout JJ, Wilson D, Grint D, Alufandika-Moyo M, et al. Rapid urine-based screening for tuberculosis in HIV-positive patients admitted to hospital in Africa (STAMP): a pragmatic, multicentre, parallelgroup, double-blind, randomised controlled trial. Lancet 2018;392(10144):292-301. [PUBMED: 30032978]

\section{Hadgu 2005}

Hadgu A, Dendukuri N, Hilden J. Evaluation of nucleic acid amplification tests in the absence of a perfect gold-standard test: a review of the statistical and epidemiologic issues. Epidemiology 2005;16(5):604-12.

\section{Haraka 2018}

Haraka F, Nathavitharana RR, Schumacher SG, Kakolwa M, Denkinger CM, Gagneux S, et al. Impact of diagnostic test Xpert MTB/RIF ${ }^{\circledR}$ on health outcomes for tuberculosis. Cochrane Database of Systematic Reviews 2018, Issue 2. [DOI: 10.1002/14651858.CD012972]

\section{Harris 2009}

Harris PA, Taylor R, Thielke R, Payne J, Gonzalez N, Conde JG. Research electronic data capture (REDCap) - A metadata-driven methodology and workflow process for providing translational research informatics support. Journal of Biomedical Informatics 2009;42(2):377-81.

\section{Hartmann 1967}

Hartmann G, Honikel KO, Knüsel F, Nüesch J. The specific inhibition of the DNA-directed RNA synthesis by rifamycin. Biochimica et Biophysica Acta 1967;145(3):843-4.

\section{Kendall 2017}

Kendall EA, Schumacher SG, Denkinger CM, Dowdy DW. Estimated clinical impact of the Xpert MTB/RIF Ultra cartridge for diagnosis of pulmonary tuberculosis: a modeling study. PLOS Medicine 2017;14(12):e1002472.

\section{Kohli 2018}

Kohli M, Schiller I, Dendukuri N, Dheda K, Denkinger CM, Schumacher SG, et al. Xpert ${ }^{\circledR}$ MTB/RIF assay for extrapulmonary tuberculosis and rifampicin resistance. Cochrane Database of Systematic Reviews 2018, Issue 8. [DOI: 10.1002/14651858.CD012768.pub2]

\section{Leeflang 2013}

Leeflang MM, Rutjes AW, Reitsma JB, Hooft L, Bossuyt PM. Variation of a test's sensitivity and specificity with disease prevalence. Canadian Medical Association Journal 2013;185(11):E537-44. [DOI: 10.1503/cmaj.121286]

\section{Lewinsohn 2017}

Lewinsohn DM, Leonard MK, LoBue PA, Cohn DL, Daley CL, Desmond E, et al. Official American Thoracic Society/Infectious Diseases Society of America/Centers for Disease Control and Prevention Clinical Practice Guidelines: Diagnosis of Tuberculosis in Adults and Children. Clinical Infectious Diseases 2017;64(2):e1-e33. [PUBMED: 27932390] 


\section{Lunn 2009}

Lunn D, Spiegelhalter D, Thomas A, Best N. The BUGS project: evolution, critique, and future directions. Statistics in Medicine 2009;28(25):3049-67.

\section{Macaskill 2010}

Macaskill P, Gatsonis C, Deeks JJ, Harbord RM, Takwoingi Y. Chapter 10: Analysing and presenting results. In: Deeks JJ, Bossuyt PM, Gatsonis C, editor(s). Cochrane Handbook for Systematic Reviews of Diagnostic Test Accuracy Version 1.0. The Cochrane Collaboration, 2010. Available from: srdta.cochrane.org/.

\section{Mupfumi 2014}

Mupfumi L, Makamure B, Chirehwa M, Sagonda T, Zinyowera S, Mason P, et al. Impact of Xpert MTB/RIF on antiretroviral therapy-associated tuberculosis and mortality: a pragmatic randomized controlled trial. Open Forum Infectious Diseases 2014;1(1):ofu038.

\section{Nakiyingi 2014}

Nakiyingi L, Moodley VM, Manabe YC, Nicol MP, Holshouser M, Armstrong DT, et al. Diagnostic accuracy of a rapid urine lipoarabinomannan test for tuberculosis in HIV-infected adults. Journal of Acquired Immune Deficiency Syndromes 2014;66:270-9.

\section{Nathavitharana 2017}

Nathavitharana RR, Cudahy PG, Schumacher SG, Steingart KR, Pai M, Denkinger CM. Accuracy of line probe assays for the diagnosis of pulmonary and multidrug-resistant tuberculosis: a systematic review and meta-analysis. European Respiratory Journal 2017;49(1):1601075. [DOI: 10.1183/13993003.01075-2016]

\section{Ngwira 2019}

Ngwira LG, Corbett EL, Khundi M, Barnes GL, Nkhoma A, Murowa M, et al. Screening for tuberculosis with Xpert MTB/RIF versus fluorescent microscopy among adults newly diagnosed with HIV in rural Malawi: a cluster randomized trial (CHEPETSA). Clinical Infectious Diseases 2019;68(7):1176-83. [DOI: 10.1093/ cid/ciy590]

\section{Pai 2018}

Pai M, Schumacher SG, Abimbola S. Surrogate endpoints in global health research: still searching for killer apps and silver bullets?. BMJ Global Health 2018;3(2):e000755.

\section{Perkins 2007}

Perkins MD, Cunningham J. Facing the crisis: improving the diagnosis of tuberculosis in the HIV era. Journal of Infectious Diseases 2007;196(Suppl 1):S15-27.

\section{Peter 2016}

Peter JG, Zijenah LS, Chanda D, Clowes P, Lesosky M, Gina P, et al. Effect on mortality of point-of-care, urine-based lipoarabinomannan testing to guide tuberculosis treatment initiation in HIV-positive hospital inpatients: a pragmatic, parallel-group, multicountry, open-label, randomised controlled trial. Lancet 2016;387(10024):1187-97. [PUBMED: 26970721]

\section{R Core Team 2016 [Computer program]}

$\mathrm{R}$ Core Team. R: A language and environment for statistical computing. www.R-project.org. Vienna: R Foundation for Statistical Computing, 2016.

\section{Reitsma 2005}

Reitsma JB, Glas AS, Rutjes AW, Scholten RJ, Bossuyt PM, Zwinderman $\mathrm{AH}$. Bivariate analysis of sensitivity and specificity produces informative summary measures in diagnostic reviews. Journal of Clinical Epidemiology 2005;58(10):982-90.

\section{Review Manager 2014 [Computer program]}

The Nordic Cochrane Centre, The Cochrane Collaboration. Review Manager (RevMan) [Computer program]. Version 5.3. Copenhagen: The Nordic Cochrane Centre, The Cochrane Collaboration, 2014.

\section{Schumacher 2016}

Schumacher SG, Sohn H, Qin ZZ, Gore G, Davis JL, Denkinger CM, et al. Impact of molecular diagnostics for tuberculosis on patient-important outcomes: a systematic review of study methodologies. PLoS One 2016;11(3):e015107.

\section{Schünemann 2008}

Schünemann HJ, Oxman AD, Brozek J, Glasziou P, Jaeschke R, Vist GE, et al. Grading quality of evidence and strength of recommendations for diagnostic tests and strategies. $B M J$ 2008;336(7653):1106-10.

\section{Schünemann 2016}

Schünemann HJ, Mustafa R, Brozek J, Santesso N, AlonsoCoello P, Guyatt G, et al. GRADE Working Group. GRADE Guidelines: 16. GRADE evidence to decision frameworks for tests in clinical practice and public health. Journal of Clinical Epidemiology 2016;76:89-98. [DOI: 10.1016/ j.jclinepi.2016.01.032]

\section{Shah 2016}

Shah M, Hanrahan C, Wang ZY, Dendukuri N, Lawn SD, Denkinger CM, et al. Lateral flow urine lipoarabinomannan assay for detecting active tuberculosis in HIV-positive adults. Cochrane Database of Systematic Reviews 2016, Issue 5. [DOI: 10.1002/14651858.CD011420.pub2]

\section{Small 2011}

Small PM, Pai M. Tuberculosis diagnosis - time for a game change. New England Journal of Medicine 2011;363(111):1070-1.

\section{Stata 2017 [Computer program]}

StataCorp. StataCorp LP. Stata Statistical Software Release 15. College Station, Texas: StataCorp. StataCorp LP, 2017.

\section{Steingart 2006a}

Steingart KR, Henry M, Ng V, Hopewell PC, Ramsay A, Cunningham J, et al. Fluorescence versus conventional sputum smear microscopy for tuberculosis: a systematic review. Lancet Infectious Diseases 2006;6(9):570-81.

\section{Steingart 2006b}

Steingart KR, Ng V, Henry M, Hopewell PC, Ramsay A, Cunningham J, et al. Sputum processing methods to improve

Xpert MTB/RIF and Xpert MTB/RIF Ultra for pulmonary tuberculosis and rifampicin resistance in adults (Review) 
the sensitivity of smear microscopy for tuberculosis: a systematic review. Lancet Infectious Diseases 2006;6(10):664-74.

\section{Steingart 2015}

Steingart KR, Schiller I, Dendukuri N, Lalli M, Houben R, Churchyard G, et al. In reply to 'False-positive Xpert ${ }^{\circledR}$ MTB/RIF assays in previously treated patients'. International Journal of Tuberculosis and Lung Disease 2015;19(3):366-7.

\section{Takwoingi 2013}

Takwoingi Y, Leeflang MM, Deeks JJ. Empirical evidence of the importance of comparative studies of diagnostic test accuracy. Annals of Internal Medicine 2013;158(7):544-54.

\section{Telenti 1993}

Telenti A, Imboden P, Marchesi F, Lowrie D, Cole S, Colston MJ, et al. Detection of rifampicin-resistance mutations in Mycobacterium tuberculosis. Lancet 1993;341(8846):647-50.

\section{Theron 2014c}

Theron G, Peter J, Dowdy D, Langley I, Squire SB, Dheda K. Do high rates of empirical treatment undermine the potential effect of new diagnostic tests for tuberculosis in high-burden settings?. Lancet Infectious Diseases 2014;14(6):527-32.

\section{Toman 2004a}

Toman K. How many bacilli are present in a sputum specimen found positive by smear microscopy?. In: Frieden T editor(s). Toman's Tuberculosis: Case Detection, Treatment, and Monitoring - Questions and Answers. WHO/HTM/TB/2004.334. 2nd Edition. Geneva: World Health Organization, 2004:11-13.

\section{Toman 2004b}

Toman K. What is the probability of obtaining a negative culture from a sputum specimen found positive by smear microscopy?. In: Frieden T editor(s). Toman's Tuberculosis: Case Detection, Treatment, and Monitoring - Questions and Answers. WHO/HTM/TB/2004.33. 2nd Edition. Geneva: World Health Organization, 2004:44-5.

\section{Trajman 2015}

Trajman A, Durovni B, Saraceni V, Menezes A, CordeiroSantos M, Cobelens F, et al. Impact on patients' treatment outcomes of Xpert MTB/RIF implementation for the diagnosis of tuberculosis: follow-up of a stepped-wedge randomized clinical trial. PLoS One 2015;10(4):e0123252.

\section{Unitaid 2017}

Boyle D. Tuberculosis Diagnostics Technology and Market Landscape. 5th Edition. Vernier: World Health Organization Unitaid Secretariat, 2017.

\section{Walzl 2018}

Walzl G, McNerney R, Du Plessis N, Bates M, McHugh TD, Chegou NN, et al. Tuberculosis: advances and challenges in development of new diagnostics and biomarkers. Lancet Infectious Diseases 2018;18(7):e199-e210.

\section{Whiting 2011}

Whiting PF, Rutjes AW, Westwood ME, Mallett S, Deeks JJ, Reitsma JB, et al. QUADAS-2: a revised tool for the quality assessment of diagnostic accuracy studies. Annals of Internal Medicine 2011;155(8):529-36.

\section{WHO 2016a}

World Health Organization. Xpert MTB/RIF assay for the diagnosis of TB: meeting report. apps.who.int/iris/ handle/10665/250383 (accessed 30 November 2018).

\section{WHO 2016b}

World Health Organization. WHO Treatment guidelines for drugresistant tuberculosis, 2016 update. WHO/HTM/TB/2016.04. Geneva: World Health Organization October 2016.

\section{WHO Compendium 2018}

WHO. Compendium of WHO Guidelines and Associated Standards: Ensuring Optimum Delivery of the Cascade of Care for Patients with Tuberculosis. 2nd Edition. Geneva: World Health Organization, 2018.

\section{WHO Global TB Report 2018}

World Health Organization. Global Tuberculosis Report 2018. Licence: CC BY-NC-SA 3.0 IGO. Geneva: World Health Organization, 2018.

\section{WHO LAM 2015}

World Health Organization. The use of lateral flow urine lipoarabinomannan assay (LF-LAM) for the diagnosis and screening of active tuberculosis in people living with HIV. Policy guidance. WHO/HTM/TB/2015.25. Geneva: World Health Organization 2015.

\section{WHO LPA 2016}

World Health Organization. The use of molecular line probe assays for the detection of resistance to isoniazid and rifampicin: policy update. WHO/HTM/TB/2016.12. Geneva: World Health Organization 2016.

\section{WHO Policy DST 2008}

World Health Organization. Policy Guidance on DrugSusceptibility Testing (DST) of Second-Line Antituberculosis Drugs. WHO/HTM/TB/2008.392. Geneva: World Health Organization, 2008.

\section{WHO Policy Framework 2015}

World Health Organization. Implementing tuberculosis diagnostics: A policy framework. WHO/HTM/TB/2015.11. Geneva: World Health Organization, 2015.

\section{WHO Policy Smear-positive TB Case 2007}

World Health Organization. Definition of a new sputum smear-positive TB case. www.who.int/tb/laboratory/ policy_sputum_smearpositive_tb_case/en/index.html 2007 (accessed 8 May 2019).

\section{WHO Policy Xpert MTB/RIF 2011}

World Health Organization. Policy Statement: Automated Real-Time Nucleic Acid Amplification Technology for Rapid and Simultaneous Detection of Tuberculosis and Rifampicin Resistance: Xpert MTB/RIF system. WHO/HTM/TB/2011.4. Geneva: World Health Organization, 2011. 


\section{WHO Rapid Implementation 2011}

World Health Organization. Rapid Implementation of the Xpert MTB/RIF Diagnostic Test. Technical and Operational 'How-to'. Practical Considerations. WHO/HTM/TB/2011.2. Geneva: World Health Organization, 2011.

\section{WHO Xpert MTB/RIF Policy Update 2013}

World Health Organization. Automated Real-Time Nucleic Acid Amplification Technology for Rapid and Simultaneous Detection of Tuberculosis and Rifampicin Resistance: Xpert MTB/RIF System for the Diagnosis of Pulmonary and Extrapulmonary TB in Adults and Children: Policy Update. WHO/ HTM/TB/2013.14. Geneva: World Health Organization, 2013.

\section{WHO Xpert Ultra 2017}

World Health Organization. WHO meeting report of a technical expert consultation: non-inferiority analysis of Xpert MTB/ RIF Ultra compared to Xpert MTB/RIF. WHO/HTM/TB/2017.04. Geneva: WHO 2017.

\section{World Bank 2017}

World Bank. World Bank List of Economies. Washington (District of Columbia): World Bank, 2017.

\section{CHARACTERISTICS OF STUDIES}

Characteristics of included studies [ordered by study ID]

\section{Zumla 2012}

Zumla A, Abubakar I, Raviglione M, Hoelscher M, Ditiu L, McHugh TD, et al. Drug-resistant tuberculosis--current dilemmas, unanswered questions, challenges, and priority needs. Journal of Infectious Diseases 2012;205(Suppl 2):S228-40.

\section{References to other published versions of this review \\ Sohn 2012}

Sohn H, Pai M, Dendukuri N, Kloda LA, Boehme CC, Steingart KR. Xpert MTB/RIF test for detection of pulmonary tuberculosis and rifampicin resistance. Cochrane Database of Systematic Reviews 2012, Issue 1. [DOI: 10.1002/14651858.CD009593]

\section{Steingart 2013}

Steingart KR, Sohn H, Schiller I, Kloda LA, Boehme CC, Pai M, et al. Xpert ${ }^{\circledR}$ MTB/RIF assay for pulmonary tuberculosis and rifampicin resistance in adults. Cochrane Database of Systematic Reviews 2013, Issue 1. [DOI: 10.1002/14651858.CD009593.pub2]

\section{Steingart 2014}

Steingart KR, Schiller I, Horne DJ, Pai M, Boehme CC, Dendukuri N. Xpert ${ }^{\circledR}$ MTB/RIF assay for pulmonary tuberculosis and rifampicin resistance in adults. Cochrane Database of Systematic Reviews 2014, Issue 1. [DOI: 10.1002/14651858.CD009593.pub3]

Adelman 2015

\section{Study characteristics}

Patient sampling

Cross-sectional design, consecutive enrolment, prospective data collection

Patient characteristics and setting

Presenting signs and symptoms: people with at least one of the following: cough, fever, night sweats, and weight loss

Age: 18 years and older

Sex, female: not reported

HIV infection: $100 \%$

History of TB: $36 \%$

Sample size: 212

Clinical setting: outpatient

Laboratory level: intermediate

Country: Ethiopia

World Bank Income Classification: low income

High TB burden country: yes

High MDR-TB burden country: yes 
Adelman 2015 (Continued)

High TB/HIV burden country: yes

Prevalence of TB cases in the study: $2.8 \%$

\begin{tabular}{ll}
\hline Index tests & Index: Xpert MTB/RIF \\
\hline Target condition and reference standard(s) & Target condition: pulmonary TB \\
& Reference standard for pulmonary TB: LJ
\end{tabular}

Flow and timing

Comparative

$$
\text { Notes }
$$

\section{Methodological quality}

\begin{tabular}{lll}
\hline Item & $\begin{array}{l}\text { Authors' judge- } \\
\text { ment }\end{array}$ & $\begin{array}{c}\text { Risk of bias } \\
\text { Applicability } \\
\text { concerns }\end{array}$ \\
\hline DOMAIN 1: Patient Selection & Yes & Yes \\
\hline Was a consecutive or random sample of patients enrolled? & Yes & Low \\
\hline Was a case-control design avoided? & Low
\end{tabular}

DOMAIN 2: Index Test Xpert MTB/RIF

Were the index test results interpreted without knowledge of the results Yes of the reference standard?

If a threshold was used, was it pre-specified?

Yes

\begin{tabular}{ll}
\hline & Low \\
\hline DOMAIN 3: Reference Standard & Low \\
\hline $\begin{array}{l}\text { Is the reference standards likely to correctly classify the target condi- } \\
\text { tion? }\end{array}$ & Yes
\end{tabular}

Were the reference standard results for TB detection interpreted with- Unclear out knowledge of the results of the index test?

Were the reference standard results for rifampicin resistance detection interpreted without knowledge of the results of the index test?

Unclear Unclear

DOMAIN 4: Flow and Timing

Was there an appropriate interval between index test and reference Yes standard? 
Adelman 2015 (Continued)

Did all patients receive the same reference standard?

Yes

Were all patients included in the analysis?

Yes

Low

Al-Darraji 2013

\section{Study characteristics}

Patient sampling

Cross-sectional design, consecutive enrolment, prospective data collection

\section{Patient characteristics and setting}

Presenting signs and symptoms: not reported; HIV-positive prisoners were screened

Age: mean 37 years (standard deviation (SD) 6.6)

Sex, female: $10 \%$

HIV infection: $100 \%$

History of TB: $29 \%$

Sample size: 125

Clinical setting: outpatient, point of care

Laboratory level: other, prison

Country: Malaysia

World Bank Income Classification: middle income

High TB burden country: no

High MDR-TB burden country: no

High TB/HIV burden country: no

Prevalence of TB cases in the study: $12.0 \%$

Index tests Index: Xpert MTB/RIF

Target condition and reference standard(s)

Target condition: pulmonary TB

Reference standard for pulmonary TB: MGIT 960

Target condition: rifampicin resistance

Reference standard for rifampicin resistance: MGIT 960, MTBDRplus for confirmation

Flow and timing

Comparative

Notes 
Al-Darraji 2013 (Continued)

Methodological quality

\begin{tabular}{|c|c|c|c|}
\hline Item & $\begin{array}{l}\text { Authors' judge- } \\
\text { ment }\end{array}$ & Risk of bias & $\begin{array}{l}\text { Applicability con- } \\
\text { cerns }\end{array}$ \\
\hline
\end{tabular}

\section{DOMAIN 1: Patient Selection}

\begin{tabular}{lcc}
\hline Was a consecutive or random sample of patients enrolled? & Yes \\
\hline Was a case-control design avoided? & Yes & \\
\hline Did the study avoid inappropriate exclusions? & Yes & Low \\
\hline
\end{tabular}

DOMAIN 2: Index Test Xpert MTB/RIF

Were the index test results interpreted without knowledge of the Yes results of the reference standard?

If a threshold was used, was it pre-specified? Yes

\begin{tabular}{|c|c|c|}
\hline & Low & Low \\
\hline \multicolumn{3}{|l|}{ DOMAIN 3: Reference Standard } \\
\hline $\begin{array}{l}\text { Is the reference standards likely to correctly classify the target } \\
\text { condition? }\end{array}$ & Yes & \\
\hline $\begin{array}{l}\text { Were the reference standard results for TB detection interpreted } \\
\text { without knowledge of the results of the index test? }\end{array}$ & Yes & \\
\hline \multirow{2}{*}{$\begin{array}{l}\text { Were the reference standard results for rifampicin resistance de- } \\
\text { tection interpreted without knowledge of the results of the index } \\
\text { test? }\end{array}$} & Yes & \\
\hline & Low & Low \\
\hline \multicolumn{3}{|l|}{ DOMAIN 4: Flow and Timing } \\
\hline $\begin{array}{l}\text { Was there an appropriate interval between index test and refer- } \\
\text { ence standard? }\end{array}$ & Yes & \\
\hline Did all patients receive the same reference standard? & Yes & \\
\hline \multirow[t]{2}{*}{ Were all patients included in the analysis? } & Yes & \\
\hline & Low & \\
\hline
\end{tabular}

\section{Ali 2017}

\section{Study characteristics}




\section{Ali 2017 (Continued)}

Patient sampling

Patient characteristics and setting

Cross-sectional design, unclear manner of enrolment, retrospective data collection

Presenting signs and symptoms: people with pulmonary TB, recently found to have smear-positive sputum

Age:

$\leq 15$ years $1(0.8 \%)$

16 to $3081(64.3 \%)$

31 to $4523(18.2 \%)$

46 to $6015(11.9 \%)$

$\geq 606(4.8 \%)$

Sex, female: $33 \%$

HIV infection: not reported

History of TB: $57 \%$

Sample size: 126

Clinical setting: laboratory-based

Laboratory level: central

Country: Sudan

World Bank Income Classification: middle income

High TB burden country: no

High MDR-TB burden country: no

High TB/HIV burden country: no Index: Xpert MTB/RIF

\section{Target condition: rifampicin resistance}

Reference standard for rifampicin resistance: LJ

Flow and timing

Comparative

Notes

Participants were recruited from random geographical clusters during a one-year period

\section{Methodological quality}

\begin{tabular}{|c|}
\hline Item \\
\hline
\end{tabular}

\section{DOMAIN 1: Patient Selection}

\begin{tabular}{ll}
\hline Was a consecutive or random sample of patients enrolled? & Unclear \\
\hline Was a case-control design avoided? & Yes \\
\hline Did the study avoid inappropriate exclusions? & No \\
\hline
\end{tabular}

Xpert MTB/RIF and Xpert MTB/RIF Ultra for pulmonary tuberculosis and rifampicin resistance in adults (Review) 79

Copyright ( 2019 The Authors. Cochrane Database of Systematic Reviews published by John Wiley \& Sons, Ltd. on behalf of The Cochrane

Collaboration. 
Ali 2017 (Continued)

High

Unclear

\section{DOMAIN 2: Index Test Xpert MTB/RIF}

Were the index test results interpreted without knowledge of the Yes results of the reference standard?

If a threshold was used, was it pre-specified?

Yes

\begin{tabular}{llll}
\hline Low & Low \\
\hline
\end{tabular}

\section{DOMAIN 3: Reference Standard}

Is the reference standards likely to correctly classify the target Yes condition?

Were the reference standard results for TB detection interpreted Unclear without knowledge of the results of the index test?

Were the reference standard results for rifampicin resistance detection interpreted without knowledge of the results of the index Unclear test?

\section{DOMAIN 4: Flow and Timing}

Was there an appropriate interval between index test and refer- Yes ence standard?

Did all patients receive the same reference standard? Yes

Were all patients included in the analysis? Yes

\section{Atwebembeire 2016}

\section{Study characteristics}

Patient sampling

Patient characteristics and setting
Cross-sectional design, unclear manner of enrolment, prospective data collection

\author{
Presenting signs and symptoms: people who were unable to produce \\ sputum with a clinical suspicion of TB (presence of at least 1 of the fol- \\ lowing signs: cough of at least 2 weeks, chronic unexplained weight \\ loss, fever, or recent chest $x$-ray showing radiological features compati- \\ ble with TB); specimens were frozen \\ Age: adults, mean or median age not reported \\ Sex, female: $46 \%$ \\ HIV infection: $31 \%$ \\ History of TB: not reported
}


Sample size: 104

Clinical setting: laboratory-based

Laboratory level: central

Country: Uganda

World Bank Income Classification: low income

High TB burden country: no

High MDR-TB burden country: no

High TB/HIV burden country: yes

Prevalence of TB cases in the study: $31.7 \%$

\begin{tabular}{ll}
\hline Index tests & Index: Xpert MTB/RIF \\
\hline Target condition and reference standard(s) & Target condition: pulmonary TB \\
& Reference standard for pulmonary TB: LJ and MGIT 960
\end{tabular}

Flow and timing

\section{Comparative}

Notes

Frozen sediments of sputum specimens previously evaluated using MGIT and LJ were used in this study.

\section{Methodological quality}

\begin{tabular}{llll}
\hline Item & Authors' judgement & Risk of bias & $\begin{array}{l}\text { Applicability con- } \\
\text { cerns }\end{array}$
\end{tabular}

\section{DOMAIN 1: Patient Selection}

\begin{tabular}{llll}
\hline $\begin{array}{l}\text { Was a consecutive or random sample of patients en- } \\
\text { rolled? }\end{array}$ & Unclear \\
\hline Was a case-control design avoided? & Yes & Yes & Unclear \\
\hline Did the study avoid inappropriate exclusions? & & Unclear
\end{tabular}

\section{DOMAIN 2: Index Test Xpert MTB/RIF}

Were the index test results interpreted without knowledge Yes

of the results of the reference standard?

If a threshold was used, was it pre-specified? Yes

Low

Low

\section{DOMAIN 3: Reference Standard}

Is the reference standards likely to correctly classify the

Yes

target condition?

Xpert MTB/RIF and Xpert MTB/RIF Ultra for pulmonary tuberculosis and rifampicin resistance in adults (Review) 
Atwebembeire 2016 (Continued)

Were the reference standard results for TB detection inter- Unclear preted without knowledge of the results of the index test?

Were the reference standard results for rifampicin resis-

tance detection interpreted without knowledge of the re-

sults of the index test?

Unclear

Low

\section{DOMAIN 4: Flow and Timing}

Was there an appropriate interval between index test and Yes reference standard?

Did all patients receive the same reference standard? Yes

Were all patients included in the analysis? Yes

Low

\section{Balcells 2012}

\section{Study characteristics}

Patient sampling

Cross-sectional design, consecutive enrolment, prospective data collection

\section{Patient characteristics and setting}

Presenting signs and symptoms: people who fulfilled at least 1 of the following criteria: cough (> 10 days), bloody sputum, pneumonia unresponsive to previous antibiotics, fever ( $>10$ days), abnormal CXR or weight loss

Age: mean 37.4 years, range 19 - 65 years

Sex, female: $20.6 \%$

HIV infection: $100 \%$

History of TB: $11.8 \%$

Sample size: 160

Clinical setting: 5 hospitals and their respective HIV clinics

Laboratory level: central

Country: Chile

World Bank Income Classification: middle income

High TB burden country: no

High MDR-TB burden country: no

High TB/HIV burden country: no

TB incidence rate: 18 per 100,000 
Balcells 2012 (Continued)

MDR-TB prevalence: percentage MDR-TB among new TB cases $=0.7 \%$

(Source: nationwide survey 2001) and among retreatment cases $=3.2 \%$

(Source: nationwide surveillance 2011)

Prevalence of TB cases in the study: $7.5 \%$

\section{Index tests}

Index: Xpert MTB/RIF

Target condition and reference standard(s)

Target condition: pulmonary TB

Reference standard for pulmonary TB: LJ and MGIT 960

Target condition: rifampicin resistance

Reference standard for rifampicin resistance: proportion method on LJ media

\section{Flow and timing}

Comparative

\section{Notes}

\section{Methodological quality}

\begin{tabular}{llll}
\hline Item & Authors' judgement & Risk of bias & $\begin{array}{l}\text { Applicability con- } \\
\text { cerns }\end{array}$ \\
\hline
\end{tabular}

\section{DOMAIN 1: Patient Selection}

Was a consecutive or random sample of patients en- Yes rolled?

\begin{tabular}{lc}
\hline Was a case-control design avoided? & Yes \\
\hline Did the study avoid inappropriate exclusions? & Yes
\end{tabular}

\begin{tabular}{lll}
\hline Low & Low \\
\hline
\end{tabular}

\section{DOMAIN 2: Index Test Xpert MTB/RIF}

Were the index test results interpreted without knowl- Yes edge of the results of the reference standard?

If a threshold was used, was it pre-specified? Yes

\begin{tabular}{lll}
\hline Low & Low \\
\hline
\end{tabular}

\section{DOMAIN 3: Reference Standard}

Is the reference standards likely to correctly classify the Yes target condition?

Were the reference standard results for TB detection in- Yes terpreted without knowledge of the results of the index test? 
Balcells 2012 (Continued)

Were the reference standard results for rifampicin resistance detection interpreted without knowledge of the results of the index test?

\section{Low}

Low

\section{DOMAIN 4: Flow and Timing}

Was there an appropriate interval between index test Yes and reference standard?

Did all patients receive the same reference standard? Yes

Were all patients included in the analysis? Yes

Low

\section{Balcha 2014}

\section{Study characteristics}

Patient sampling

Cross-sectional design, consecutive enrolment, prospective data collection

Patient characteristics and setting

Presenting signs and symptoms: HIV-positive people screened for TB irrespective of symptoms

Age: 18 years and older, median 32 years (IQR 28 to 40 )

Sex, female: $59 \%$

HIV infection: $100 \%$

History of TB: $6 \%$

Sample size: 810

Clinical setting: outpatient

Laboratory level: intermediate

Country: Ethiopia

World Bank Income Classification: low income

High TB burden country: yes

High MDR-TB burden country: yes

High TB/HIV burden country: yes

Prevalence of TB cases in the study: $15.0 \%$

Index tests Index: Xpert MTB/RIF

Target condition and reference standard(s)

Target condition: pulmonary TB

Reference standard for pulmonary TB: MGIT 960 
Balcha 2014 (Continued)

Flow and timing

\section{Comparative}

Notes

$2 \%$ of participants were on anti-TB treatment for up to 2 weeks

\section{Methodological quality}

\begin{tabular}{|c|c|c|c|}
\hline Item & $\begin{array}{l}\text { Authors' judge- } \\
\text { ment }\end{array}$ & Risk of bias & $\begin{array}{l}\text { Applicability con- } \\
\text { cerns }\end{array}$ \\
\hline
\end{tabular}

\section{DOMAIN 1: Patient Selection}

\begin{tabular}{lcc}
\hline Was a consecutive or random sample of patients enrolled? & Yes & Yes \\
\hline Was a case-control design avoided? & Yes & Low L L L L L L
\end{tabular}

\section{DOMAIN 2: Index Test Xpert MTB/RIF}

Were the index test results interpreted without knowledge of the re- Yes sults of the reference standard?

\begin{tabular}{lccc}
\hline If a threshold was used, was it pre-specified? & Yes & Low & Unclear \\
\hline
\end{tabular}

\section{DOMAIN 3: Reference Standard}

Is the reference standards likely to correctly classify the target condi- Yes tion?

Were the reference standard results for TB detection interpreted Yes without knowledge of the results of the index test?

Were the reference standard results for rifampicin resistance detec- Yes tion interpreted without knowledge of the results of the index test?

Low Low

\section{DOMAIN 4: Flow and Timing}

Was there an appropriate interval between index test and reference Yes standard?

\begin{tabular}{ll}
\hline Did all patients receive the same reference standard? & Yes \\
\hline Were all patients included in the analysis? & Yes \\
\hline
\end{tabular}


Barmankulova 2015

\section{Study characteristics}

\section{Patient sampling}

Patient characteristics and setting

Cross-sectional design, unknown manner of enrolment, direction of data collection unclear

Presenting signs and symptoms: at least 2 weeks of cough, accompanied with loss of weight, night sweats and fever in labour migrants

Age: median 34 years (IQR 25 to 45 )

Sex, female: $43 \%$

HIV infection: not reported

History of TB: $25 \%$

Sample size: 291

Clinical setting: outpatient

Laboratory level: intermediate and central

Country: Kyrgyzstan

World Bank Income Classification: middle income

High TB burden country: no

High MDR-TB burden country: yes

High TB/HIV burden country: no

Prevalence of TB cases in the study: $80.8 \%$

\begin{tabular}{l} 
Index tests \\
\hline Target condition and reference standard(s)
\end{tabular}

Index: Xpert MTB/RIF

Target condition: pulmonary TB

Reference standard for pulmonary TB: LJ and MGIT 960

Target condition: rifampicin resistance

Reference standard for rifampicin resistance LJ and MGIT 960

\section{Flow and timing}

43 participants without microscopy results and 3415 participants without culture results were not included

\section{Comparative}

Notes

"Migrants in the TB REACH project are defined as labour migrants who registered in one region but are working and living permanently in another region without registration and any access to primary healthcare facilities."

\section{Methodological quality}

\section{Item}

Authors' judgement

Risk of bias

Applicability con-

\section{cerns}

\section{DOMAIN 1: Patient Selection}


Barmankulova 2015 (Continued)

Was a consecutive or random sample of patients en- Unclear rolled?

\begin{tabular}{lc}
\hline Was a case-control design avoided? & Yes \\
\hline Did the study avoid inappropriate exclusions? & Yes \\
\hline
\end{tabular}

\begin{tabular}{llll}
\hline DOMAIN 2: Index Test Xpert MTB/RIF & Unclear & Low \\
\hline $\begin{array}{l}\text { Were the index test results interpreted without knowl- } \\
\text { edge of the results of the reference standard? }\end{array}$ & & \\
\hline If a threshold was used, was it pre-specified? & Yes & Low & Low
\end{tabular}

\section{DOMAIN 3: Reference Standard}

Is the reference standards likely to correctly classify Yes the target condition?

Were the reference standard results for TB detection Yes interpreted without knowledge of the results of the index test?

Were the reference standard results for rifampicin resistance detection interpreted without knowledge of the results of the index test?

Yes

\begin{tabular}{llll}
\hline DOMAIN 4: Flow and Timing & Low & Unclear \\
\hline $\begin{array}{l}\text { Was there an appropriate interval between index test } \\
\text { and reference standard? }\end{array}$ & Yes & \\
\hline Did all patients receive the same reference standard? & Yes & High \\
\hline Were all patients included in the analysis? & No & & Hig \\
\hline
\end{tabular}

\section{Barnard 2015}

\section{Study characteristics}

$\begin{array}{ll}\text { Patient sampling } & \begin{array}{l}\text { Cross-sectional design, consecutive enrolment, retrospective data collec- } \\ \text { tion }\end{array}\end{array}$

Patient characteristics and setting

Presenting signs and symptoms: presumed pulmonary TB defined as 2 of the following: HIV infection, persistent cough lasting $>3$ weeks, haemoptysis, weight loss $>4 \mathrm{~kg}$, intermittent fever $>3$ weeks or drenching night sweats $>2$ weeks. In addition, at least 1 of the following radiological cri- 
Barnard 2015 (Continued)

teria had to be present: cavitation, diffuse infiltrates, hilar or mediastinal adenopathy, primarily smear-negative

Age: 44 years (SD 16)

Sex, female: $52 \%$

HIV infection: not reported

History of TB: yes, \% not reported

Sample size: 112

Clinical setting: not reported

Laboratory level: central

Country: South Africa

World Bank Income Classification: middle income

High TB burden country: yes

High MDR-TB burden country: yes

High TB/HIV burden country: yes

Prevalence of TB cases in the study: $34.8 \%$

Index tests
Target condition and reference standard(s)

Index: Xpert MTB/RIF

Target condition: pulmonary TB

Reference standard for pulmonary TB: MGIT 960

Target condition: rifampicin resistance

Reference standard for rifampicin resistance: MTBDRplus

Flow and timing

72 participants were excluded due to incomplete data

Comparative

Notes

\section{Methodological quality}

\begin{tabular}{llll}
\hline Item & Authors' judgement & Risk of bias & $\begin{array}{l}\text { Applicability con- } \\
\text { cerns }\end{array}$ \\
\hline DOMAIN 1: Patient Selection & & \\
\hline $\begin{array}{l}\text { Was a consecutive or random sample of patients en- } \\
\text { rolled? }\end{array}$ & Yes & & \\
\hline Was a case-control design avoided? & Yes & High & Unclear \\
\hline Did the study avoid inappropriate exclusions? & No & \\
\hline
\end{tabular}

\section{DOMAIN 2: Index Test Xpert MTB/RIF}

Xpert MTB/RIF and Xpert MTB/RIF Ultra for pulmonary tuberculosis and rifampicin resistance in adults (Review) 
Barnard 2015 (Continued)

Were the index test results interpreted without knowl- Yes edge of the results of the reference standard?

If a threshold was used, was it pre-specified?

Yes

Low

Low

\section{DOMAIN 3: Reference Standard}

Is the reference standards likely to correctly classify Yes

the target condition?

Were the reference standard results for TB detection Yes

interpreted without knowledge of the results of the

index test?

Were the reference standard results for rifampicin resistance detection interpreted without knowledge of

Unclear the results of the index test?

\begin{tabular}{lll}
\hline Unclear & Low \\
\hline
\end{tabular}

DOMAIN 4: Flow and Timing

Was there an appropriate interval between index test Yes and reference standard?

Did all patients receive the same reference standard? Yes

Were all patients included in the analysis? No

High

\section{Bates 2013a}

\section{Study characteristics}

Patient sampling

Cross-sectional design, unknown manner of enrolment, prospective data collection

Patient characteristics and setting

Presenting signs and symptoms: people with cough and ability to produce a sputum sample who presented to obstetrics or gynaecology wards

Age: median 28 years (IQR 24 to 32 )

Sex, female: $100 \%$

HIV infection: $66 \%$

History of TB: 12\%

Sample size: 94

Clinical setting: inpatient

Laboratory level: central 
Bates 2013a (Continued)

Country: Zambia

World Bank Income Classification: middle income

High TB burden country: yes

High MDR-TB burden country: no

High TB/HIV burden country: yes

Prevalence of TB cases in the study: $27.7 \%$

\begin{tabular}{ll}
\hline Index tests & Index: Xpert MTB/RIF \\
\hline Target condition and reference standard(s) & Target condition: pulmonary TB \\
& $\begin{array}{l}\text { Reference standard for pulmonary TB: MGIT 960 } \\
\text { Target condition: rifampicin resistance }\end{array}$ \\
\hline Flow and timing & Reference standard for rifampicin resistance: MGIT 960 \\
\hline Comparative & \\
\hline Notes & $\begin{array}{l}\text { Risk of bias } \\
\text { Methodological quality }\end{array}$ \\
\hline Item & $\begin{array}{l}\text { Applicability con- } \\
\text { cerns }\end{array}$
\end{tabular}

\section{DOMAIN 1: Patient Selection}

\begin{tabular}{llll}
\hline Was a consecutive or random sample of patients enrolled? & Unclear \\
\hline Was a case-control design avoided? & Yes & Yes & Unclear \\
\hline Did the study avoid inappropriate exclusions? & & High
\end{tabular}

\section{DOMAIN 2: Index Test Xpert MTB/RIF}

Were the index test results interpreted without knowledge of the Yes results of the reference standard?

\begin{tabular}{lcc}
\hline If a threshold was used, was it pre-specified? & Yes \\
\hline Low & Low \\
\hline
\end{tabular}

\section{DOMAIN 3: Reference Standard}

Is the reference standards likely to correctly classify the target Yes condition?

Were the reference standard results for TB detection interpreted Yes without knowledge of the results of the index test? 
Bates 2013a (Continued)

Were the reference standard results for rifampicin resistance detection interpreted without knowledge of the results of the index test?

Low

Low

\section{DOMAIN 4: Flow and Timing}

Was there an appropriate interval between index test and refer- Yes ence standard?

Did all patients receive the same reference standard? Yes

Were all patients included in the analysis? Yes

Low

\section{Bjerrum 2016}

\section{Study characteristics}

Patient sampling

Cross-sectional design, consecutive enrolment, prospective data collection

Patient characteristics and setting

Presenting signs and symptoms: HIV-infected adults screened for pulmonary TB irrespective of symptoms

Age: 18 years and older, median 38 years (IQR 31 to 45 )

Sex, female: $64 \%$

HIV infection: $100 \%$

History of TB: $6 \%$

Sample size: 195

Clinical setting: both outpatient and inpatient

Laboratory level: central

Country: Ghana

World Bank Income Classification: middle income

High TB burden country: no

High MDR-TB burden country: no

High TB/HIV burden country: yes

Prevalence of TB cases in the study: 17.9\%

\begin{tabular}{ll}
\hline Index tests & Index: Xpert MTB/RIF \\
\hline Target condition and reference standard(s) & Target condition: pulmonary TB \\
& Reference standard for pulmonary TB: LJ and MGIT 960
\end{tabular}


Bjerrum 2016 (Continued)

Flow and timing

\section{Comparative}

Notes Screening study

\section{Methodological quality}

\begin{tabular}{|c|c|c|c|}
\hline Item & $\begin{array}{l}\text { Authors' judge- } \\
\text { ment }\end{array}$ & Risk of bias & $\begin{array}{l}\text { Applicability } \\
\text { concerns }\end{array}$ \\
\hline
\end{tabular}

\section{DOMAIN 1: Patient Selection}

\begin{tabular}{lcc} 
Was a consecutive or random sample of patients enrolled? & Yes & Yes \\
\hline Was a case-control design avoided? & Yes & Low \\
\hline Did the study avoid inappropriate exclusions? & Low \\
\hline
\end{tabular}

\section{DOMAIN 2: Index Test Xpert MTB/RIF}

Were the index test results interpreted without knowledge of the re- Yes sults of the reference standard?

\section{DOMAIN 3: Reference Standard}

Is the reference standards likely to correctly classify the target condi- Yes tion?

Were the reference standard results for TB detection interpreted with- Yes out knowledge of the results of the index test?

Were the reference standard results for rifampicin resistance detection interpreted without knowledge of the results of the index test?

\begin{tabular}{lll}
\hline & Low & Low \\
\hline DOMAIN 4: Flow and Timing & Yes \\
\hline $\begin{array}{l}\text { Was there an appropriate interval between index test and reference } \\
\text { standard? }\end{array}$ & Yes \\
\hline Did all patients receive the same reference standard? & Yes \\
\hline Were all patients included in the analysis? &
\end{tabular}


Boehme 2010

\section{Study characteristics}

Patient sampling

Cross-sectional design, consecutive enrolment, prospective data collection, site in a multicentre study

Patient characteristics and setting

Presenting signs and symptoms: persistent productive cough for $\geq 2$ weeks

Age: median 34 years, range 17 to 88 years

Sex, female: $37 \%$

HIV infection: $40 \%$

History of TB: $46 \%$

Sample size: 1730

Clinical setting: special facility for prisoners (Azerbaijan); primary health care DOTS (directly observed treatment, short-course) centres in shanty towns (Peru); clinic (South Africa, Cape Town); TB clinics (South Africa, Durban); tertiary hospital (India)

Laboratory level: central

Country: Azerbaijan, India, Peru, South Africa

World Bank Income Classification: middle income

High TB burden country: yes (India, South Africa)

High MDR-TB burden country: yes (Azerbaijan, India, Peru, South Africa)

High TB/HIV burden country: yes (India, South Africa)

Prevalence of TB cases in study: 50.9\%

\begin{tabular}{ll}
\hline Index tests & Index: Xpert MTB/RIF \\
\hline Target condition and reference standard(s) & Target condition: pulmonary TB \\
& $\begin{array}{l}\text { Reference standard for pulmonary TB: LJ culture. 7H11 culture, and MGIT } \\
960\end{array}$ \\
& Target condition: rifampicin resistance \\
& $\begin{array}{l}\text { Reference standard for rifampicin resistance: proportion method on LJ me- } \\
\text { dia, MGIT, MTBDRplus }\end{array}$ \\
&
\end{tabular}

Flow and timing

Comparative

Notes

Methodological quality

\begin{tabular}{llll}
\hline Item Authors' judgement & Risk of bias & $\begin{array}{l}\text { Applicability con- } \\
\text { cerns }\end{array}$ \\
\hline
\end{tabular}

DOMAIN 1: Patient Selection 
Boehme 2010 (Continued)

Was a consecutive or random sample of patients en- Yes rolled?

\begin{tabular}{lc}
\hline Was a case-control design avoided? & Yes \\
\hline Did the study avoid inappropriate exclusions? & Yes \\
\hline
\end{tabular}

\begin{tabular}{llll}
\hline DOMAIN 2: Index Test Xpert MTB/RIF & Low & Low \\
\hline $\begin{array}{l}\text { Were the index test results interpreted without knowl- } \\
\text { edge of the results of the reference standard? }\end{array}$ & & \\
\hline If a threshold was used, was it pre-specified? & Yes & Low & Low
\end{tabular}

\section{DOMAIN 3: Reference Standard}

Is the reference standards likely to correctly classify Yes the target condition?

Were the reference standard results for TB detection Yes interpreted without knowledge of the results of the index test?

Were the reference standard results for rifampicin resistance detection interpreted without knowledge of the results of the index test?

Yes

Low Low L L L L

\section{DOMAIN 4: Flow and Timing}

Was there an appropriate interval between index test Yes and reference standard?

Did all patients receive the same reference standard? Yes

Were all patients included in the analysis?

Yes

Boehme 2011

\section{Study characteristics}

Patient sampling Cross-sectional design, consecutive enrolment, prospective data collection, site in a multicentre study

Patient characteristics and setting Presenting signs and symptoms: cough lasting at least 2 weeks

Age: median 38 years (IQR 29 to 50)

Sex, female: $39 \%$ 
Boehme 2011 (Continued)

HIV infection: $19 \%$

History of TB: not reported

Sample size: 6648

Clinical setting: special facility for prisoners (Azerbaijan); 2 health centres and 1 district hospital (Peru); 1 health centre and 1 provincial hospital (South Africa, Cape Town); emergency unit of referral hospital (Uganda); health centre (India); MDR-TB evaluation facility (Philippines)

Laboratory level: central (Azerbaijan, Peru, Philippines, South Africa, Uganda); intermediate (India)

Country: Azerbaijan, India, Peru, Philippines, South Africa, Uganda

World Bank Income Classification: middle income (Azerbaijan, India, South Africa, Philippines); low income (Uganda)

High TB burden country: yes (India, Philippines, South Africa)

High MDR-TB burden country: yes (Azerbaijan, India, Peru, Philippines, South Africa)

High TB/HIV burden country: yes (India, South Africa, Uganda)

Prevalence of TB cases in the study: $26.4 \%$

\begin{tabular}{ll}
\hline Index tests & Index: Xpert MTB/RIF \\
\hline $\begin{array}{l}\text { Target condition and reference stan- } \\
\text { dard(s) }\end{array}$ & Target condition: pulmonary TB \\
& Reference standard for pulmonary TB: LJ, Ogawa, MGIT 960 \\
& Target condition: rifampicin resistance \\
& $\begin{array}{l}\text { Reference standard for rifampicin resistance: LJ proportion method; MGIT 960; MTBDR- } \\
\text { plus }\end{array}$ \\
\hline Flow and timing & $\begin{array}{l}\text { Participants who were smear-negative and culture-negative but treated for TB on the } \\
\text { basis of clinical and radiological findings (clinical tuberculosis) were not included in de- } \\
\text { termination of specificity }\end{array}$ \\
\hline Comparative & \\
\hline Notes & $\begin{array}{l}\text { Follow-up reported for all sites combined: 24/153 participants with culture-negative, } \\
\text { clinically-diagnosed TB had positive results on MTB/RIF testing. 20/24 participants had } \\
\text { follow-up, and all 20 improved on TB treatment }\end{array}$ \\
\hline
\end{tabular}

\section{Methodological quality}

\begin{tabular}{llll}
\hline Item & Authors' judgement & Risk of bias & Applicability concerns \\
\hline
\end{tabular}

\section{DOMAIN 1: Patient Selection}

Was a consecutive or random sample of Yes patients enrolled?

\begin{tabular}{ll}
\hline Was a case-control design avoided? & Yes \\
\hline $\begin{array}{l}\text { Did the study avoid inappropriate exclu- } \\
\text { sions? }\end{array}$ & Yes \\
\hline
\end{tabular}


Boehme 2011 (Continued)

\section{DOMAIN 2: Index Test Xpert MTB/RIF}

Were the index test results interpreted reference standard?

If a threshold was used, was it pre-speci- Yes

fied?

Low

Low

\section{DOMAIN 3: Reference Standard}

Is the reference standards likely to cor- Yes rectly classify the target condition?

Were the reference standard results for Yes

TB detection interpreted without knowl-

edge of the results of the index test?

Were the reference standard results for rifampicin resistance detection interpreted without knowledge of the results of the index test?

\begin{tabular}{llll}
\hline DOMAIN 4: Flow and Timing & Low & Low \\
\hline $\begin{array}{l}\text { Was there an appropriate interval be- } \\
\text { tween index test and reference standard? }\end{array}$ & Yes & \\
\hline $\begin{array}{l}\text { Did all patients receive the same refer- } \\
\text { ence standard? }\end{array}$ & Yes \\
\hline Were all patients included in the analysis? & No & High \\
\hline
\end{tabular}

Boum 2016

\section{Study characteristics}

Patient sampling

Patient characteristics and setting
Cross-sectional design, unknown manner of enrolment, prospective data collection

Presenting signs and symptoms: presumed pulmonary TB with cough for 2 weeks and at least 1 additional TB symptom (fever, weight loss, or night sweats)

Age: 18 years and older, median 35 years (IQR 29 to 43) for HIV-positive participants; median 46 years (IQR 30 to 60 ) for HIV-negative participants 
Boum 2016 (Continued)

\author{
Sex, female: $50 \%$ \\ HIV infection: $70 \%$
}

History of TB: $12 \%$

Sample size: 887

Clinical setting: both outpatient and inpatient

Laboratory level: biosafety level 3 laboratory of Epicentre/Médecins sans Frontières Mbarara Research Centre

Country: Uganda

World Bank Income Classification: low income

High TB burden country: no

High MDR-TB burden country: no

High TB/HIV burden country: yes

Prevalence of TB cases in the study: $23.8 \%$

\begin{tabular}{ll}
\hline Index tests & Index: Xpert MTB/RIF \\
\hline Target condition and reference standard(s) & Target condition: pulmonary TB \\
& Reference standard for pulmonary TB: MGIT 960 \\
\hline Flow and timing & Could not account for all patients \\
\hline Comparative & \\
\hline Notes &
\end{tabular}

\title{
Methodological quality
}

\begin{tabular}{|c|c|c|c|}
\hline Item & Authors' judgement & Risk of bias & $\begin{array}{l}\text { Applicability con- } \\
\text { cerns }\end{array}$ \\
\hline
\end{tabular}

\section{DOMAIN 1: Patient Selection}

\begin{tabular}{llll}
\hline Was a consecutive or random sample of patients enrolled? & Unclear \\
\hline Was a case-control design avoided? & Yes & \\
\hline Did the study avoid inappropriate exclusions? & Yes & Unclear & Low \\
\hline
\end{tabular}

DOMAIN 2: Index Test Xpert MTB/RIF

Were the index test results interpreted without knowledge of Yes the results of the reference standard?

If a threshold was used, was it pre-specified? Yes


Boum 2016 (Continued)

\section{DOMAIN 3: Reference Standard}

Is the reference standards likely to correctly classify the target Yes condition?

Were the reference standard results for TB detection interYes preted without knowledge of the results of the index test?

Were the reference standard results for rifampicin resistance detection interpreted without knowledge of the results of the index test?

\section{DOMAIN 4: Flow and Timing}

Was there an appropriate interval between index test and ref- Yes erence standard?

Did all patients receive the same reference standard? Yes

Were all patients included in the analysis?

No

High

\section{Calligaro 2015}

\section{Study characteristics}

Patient sampling

Cross-sectional design, consecutive enrolment, prospective data collection

Patient characteristics and setting

Presenting signs and symptoms: presumed pulmonary TB (based on suggestive pulmonary infiltrates, a history of constitutional symptoms preceding the ICU admission, or people known or suspected to be infected with HIV, irrespective of the reason for admission to the ICU)

Age: 18 years and older, median 38 (IQR 28 to 51 )

Sex, female: $40 \%$

HIV infection: $27 \%$

History of TB: yes, \% not reported

Sample size: 91

Clinical setting: inpatient

Laboratory level: central

Country: South Africa

World Bank Income Classification: middle income

High TB burden country: yes

High MDR-TB burden country: yes 
Prevalence of TB cases in the study: $12.1 \%$

\begin{tabular}{ll}
\hline Index tests & Index: Xpert MTB/RIF \\
\hline Target condition and reference standard(s) & Target condition: pulmonary TB \\
& Reference standard for pulmonary TB: MGIT 960 \\
& Target condition: rifampicin resistance \\
& Reference standard for rifampicin resistance: MGIT 960
\end{tabular}

Flow and timing

Comparative

Notes

\section{Methodological quality}

\begin{tabular}{llll}
\hline Item Authors' judgement & Risk of bias $\begin{array}{l}\text { Applicability con- } \\
\text { cerns }\end{array}$ \\
\hline
\end{tabular}

\section{DOMAIN 1: Patient Selection}

\begin{tabular}{llll}
\hline Was a consecutive or random sample of patients enrolled? & Yes \\
\hline Was a case-control design avoided? & Yes & \\
\hline Did the study avoid inappropriate exclusions? & Yes & Low & High \\
\hline
\end{tabular}

\section{DOMAIN 2: Index Test Xpert MTB/RIF}

Were the index test results interpreted without knowledge of Yes the results of the reference standard?

\begin{tabular}{llll}
\hline If a threshold was used, was it pre-specified? & Yes & \\
\hline & Low & Low \\
\hline
\end{tabular}

\section{DOMAIN 3: Reference Standard}

Is the reference standards likely to correctly classify the target Yes condition?

Were the reference standard results for TB detection interpreted without knowledge of the results of the index test?

Yes

Were the reference standard results for rifampicin resistance detection interpreted without knowledge of the results of the index test? 
Calligaro 2015 (Continued)

DOMAIN 4: Flow and Timing

Was there an appropriate interval between index test and ref- Yes erence standard?

Did all patients receive the same reference standard? Yes

Were all patients included in the analysis?

Yes

\section{Calligaro 2017}

\section{Study characteristics}

Patient sampling Randomized trial, consecutive enrolment, prospective data collection

Patient characteristics and setting

Presenting signs and symptoms: HIV-positive patients with at least one TB symptom according to predefined WHO criteria and HIV-positive patients irrespective of symptoms (in line with the WHO recommendation to screen all HIV-positive individuals for TB)

Age: 18 years or older, median 38 (IQR 32 to 47 )

Sex, female: $55 \%$

HIV infection: $58 \%$

History of TB: yes, per cent not reported

Sample size: 403

Clinical setting: outpatient

Laboratory level: in South Africa, diagnostic tests were done at the point-of-contact at the mobile van, whereas in Zimbabwe, screened and eligible participants were transported to Mabvuku Clinic and the investigations were done there

Country: Zimbabwe, South Africa

World Bank Income Classification: low and middle income

High TB burden country: yes

High MDR-TB burden country: yes

High TB/HIV burden country: yes

Prevalence of TB cases in the study: $10.4 \%$

Index tests Index: Xpert MTB/RIF

\footnotetext{
Target condition and reference standard(s)
}

Target condition: pulmonary TB

Reference standard for pulmonary TB: MGIT 960

Flow and timing 
Calligaro 2017 (Continued)

Comparative

$$
\text { Notes }
$$

\section{Methodological quality}

\begin{tabular}{llll}
\hline Item Authors' judgement & Risk of bias & $\begin{array}{l}\text { Applicability con- } \\
\text { cerns }\end{array}$ \\
\hline
\end{tabular}

\section{DOMAIN 1: Patient Selection}

Was a consecutive or random sample of patients en-

rolled?

\begin{tabular}{lll}
\hline Was a case-control design avoided? & Yes \\
\hline Did the study avoid inappropriate exclusions? & Yes & Low Low
\end{tabular}

\section{DOMAIN 2: Index Test Xpert MTB/RIF}

Were the index test results interpreted without knowledge Yes

of the results of the reference standard?

\begin{tabular}{lcl}
\hline If a threshold was used, was it pre-specified? & Yes \\
\hline Low & Low
\end{tabular}

\section{DOMAIN 3: Reference Standard}

Is the reference standards likely to correctly classify the Yes target condition?

Were the reference standard results for TB detection inter- Yes preted without knowledge of the results of the index test?

Were the reference standard results for rifampicin resistance detection interpreted without knowledge of the results of the index test?

Low

Low

\section{DOMAIN 4: Flow and Timing}

Was there an appropriate interval between index test and Yes reference standard?

Did all patients receive the same reference standard? Yes

Were all patients included in the analysis? Yes 


\section{Carriquiry 2012}

\section{Study characteristics}

Patient sampling

Cross-sectional design, consecutive enrolment, prospective data collection

Patient characteristics and setting

Presenting signs and symptoms: cough for $>10$ days with abnormal chest $x$-ray and at least 1 of the following symptoms: fever, fatigue, night sweats, haemoptysis, chest pain, or weight loss

Age: 18 years or older, median 35 years (IQR 29 to 42)

Sex, female: $27.5 \%$

HIV infection: $100 \%$

History of TB: $25 \%$

Sample size: 131

Clinical setting: both inpatient and outpatient

Laboratory level: central

Country: Peru

World Bank Income Classification: middle income

High TB burden country: no

High MDR-TB burden country: yes

High TB/HIV burden country: no

TB incidence rate: 101 per 100,000

MDR-TB prevalence: percentage MDR-TB among new TB cases $=5.3 \%$

(Source: nationwide survey 2006) and among retreatment cases $=24 \%$

(Source: nationwide survey 2006)

Prevalence of TB cases in the study: $34.4 \%$

Index tests Index: Xpert MTB/RIF

\footnotetext{
Target condition and reference standard(s)
}

\section{Target condition: pulmonary TB}

Reference standard for pulmonary TB: LJ culture and MGIT 960

Target condition: rifampicin resistance

Reference standard for rifampicin resistance: proportion method on LJ media

\section{Flow and timing}

Comparative

\section{Notes}

\section{Methodological quality}

\section{Item}


Carriquiry 2012 (Continued)

\section{DOMAIN 1: Patient Selection}

Was a consecutive or random sample of patients en- Yes rolled?

\begin{tabular}{llll}
\hline Was a case-control design avoided? & Yes & \\
\hline Did the study avoid inappropriate exclusions? & Yes & Low \\
\hline
\end{tabular}

\section{DOMAIN 2: Index Test Xpert MTB/RIF}

Were the index test results interpreted without knowl- Yes edge of the results of the reference standard?

If a threshold was used, was it pre-specified? Yes

Low Low L L L L L

\section{DOMAIN 3: Reference Standard} Is the reference standards likely to correctly classify the Yes
target condition?

Were the reference standard results for TB detection interpreted without knowledge of the results of the index test?

Were the reference standard results for rifampicin resistance detection interpreted without knowledge of the results of the index test?

\section{Yes}

Yes

\begin{tabular}{ll}
\hline LOMAIN 4: Flow and Timing & Low \\
\hline $\begin{array}{l}\text { Was there an appropriate interval between index test } \\
\text { and reference standard? }\end{array}$ & Yes \\
\hline Did all patients receive the same reference standard? & Yes \\
\hline Were all patients included in the analysis? & Yes \\
\hline
\end{tabular}

Low

Chaisson 2014

\section{Study characteristics}

Patient sampling Cross-sectional design, consecutive enrolment, prospective data collection

Patient characteristics and setting

Presenting signs and symptoms: presumed pulmonary TB 
Chaisson 2014 (Continued)

Age: adults, median 54 years (IQR 43 to 60 )

Sex, female: $23 \%$

HIV infection: $30 \%$

History of TB: not reported

Sample size: 142

Clinical setting: inpatient

Laboratory level: central

Country: USA

World Bank Income Classification: high income

High TB burden country: no

High MDR-TB burden country: no

High TB/HIV burden country: no

Prevalence of TB cases in the study: $6.3 \%$

\begin{tabular}{ll}
\hline Index tests & Index: Xpert MTB/RIF \\
\hline Target condition and reference standard(s) & $\begin{array}{l}\text { Target condition: pulmonary TB } \\
\text { Reference standard for pulmonary TB: } 7 \mathrm{H} 11 \text { and BacT/Alert MP }\end{array}$ \\
\hline Flow and timing & $\begin{array}{l}59 \text { participants ( } 25 \% \text { of eligible patients) were not tested, } 46 \text { owing to } \\
\text { insufficient quantity and } 13 \text { for the following reasons: } 6 \text { samples reject- } \\
\text { ed for culture because }>3 \text { days had elapsed since collection, } 4 \text { samples } \\
\text { that were not tested for reasons that were not documented, } 2 \text { speci- } \\
\text { mens that arrived when the Xpert machine was not operating because it } \\
\text { was undergoing routine maintenance, and } 1 \text { specimen that was not } 1 \text { of } \\
\text { the first } 2 \text { samples collected }\end{array}$
\end{tabular}

\section{Comparative}

\section{Notes}

\section{Methodological quality}

\begin{tabular}{llll}
\hline Item & Authors' judgement & Risk of bias & $\begin{array}{l}\text { Applicability con- } \\
\text { cerns }\end{array}$
\end{tabular}

\section{DOMAIN 1: Patient Selection}

Was a consecutive or random sample of patients enrolled?

Yes

\begin{tabular}{llll}
\hline Was a case-control design avoided? & Yes & \\
\hline Did the study avoid inappropriate exclusions? & Yes & Low & High \\
\hline
\end{tabular}

\section{DOMAIN 2: Index Test Xpert MTB/RIF}


Chaisson 2014 (Continued)

Were the index test results interpreted without knowledge Yes of the results of the reference standard?

\begin{tabular}{lll}
\hline If a threshold was used, was it pre-specified? & Yes & \\
\hline & Low Low L L L L
\end{tabular}

\section{DOMAIN 3: Reference Standard}

Is the reference standards likely to correctly classify the Yes target condition?

Were the reference standard results for TB detection inter- No preted without knowledge of the results of the index test?

Were the reference standard results for rifampicin resis-

tance detection interpreted without knowledge of the re-

sults of the index test?

High

Low

\section{DOMAIN 4: Flow and Timing}

Was there an appropriate interval between index test and Yes reference standard?

Did all patients receive the same reference standard? Yes

Were all patients included in the analysis? No

Unclear

\section{Chen 2017}

\section{Study characteristics}

Patient sampling

Cross-sectional design, unknown manner of enrolment, prospective data collection

Patient characteristics and setting

Presenting signs and symptoms: TB symptoms

Age: 15 years and older, median 64 years (IQR 58 to 71 )

Sex, female: $42 \%$

HIV infection: not reported

History of TB: not reported

Sample size: 733

Clinical setting: outpatient, health workers went door-todoor to identify individuals with TB symptoms and send them to the clinic

Laboratory level: intermediate

Country: China

Xpert MTB/RIF and Xpert MTB/RIF Ultra for pulmonary tuberculosis and rifampicin resistance in adults (Review) 
Chen 2017 (Continued)

World Bank Income Classification: middle income

High TB burden country: yes

High MDR-TB burden country: yes

High TB/HIV burden country: yes

Prevalence of TB cases in the study: $0.8 \%$

\begin{tabular}{ll}
\hline Index tests & Index: Xpert MTB/RIF \\
\hline Target condition and reference standard(s) & Target condition: pulmonary TB \\
& Reference standard for pulmonary TB: LJ
\end{tabular}

Flow and timing

Comparative

Notes

Methodological quality

\begin{tabular}{lll}
\hline Item & $\begin{array}{l}\text { Authors' judge- } \\
\text { ment }\end{array}$ & $\begin{array}{c}\text { Risk of bias } \\
\text { concerns }\end{array}$ \\
\hline DOMAIN 1: Patient Selection & Unclear \\
\hline Was a consecutive or random sample of patients enrolled? & Yes & Yes \\
\hline Was a case-control design avoided? & & Unclear \\
\hline Did the study avoid inappropriate exclusions? & & Low \\
\hline
\end{tabular}

\section{DOMAIN 2: Index Test Xpert MTB/RIF}

Were the index test results interpreted without knowledge of the re- Yes sults of the reference standard?

If a threshold was used, was it pre-specified? Yes

\begin{tabular}{llll} 
& Low & Low \\
\hline
\end{tabular}

\section{DOMAIN 3: Reference Standard}

Is the reference standards likely to correctly classify the target condi- Yes tion?

Were the reference standard results for TB detection interpreted without knowledge of the results of the index test?

Were the reference standard results for rifampicin resistance detection interpreted without knowledge of the results of the index test?

\begin{tabular}{ll} 
Low $\quad$ Low \\
\hline
\end{tabular}


Chen 2017 (Continued)

\section{DOMAIN 4: Flow and Timing}

Was there an appropriate interval between index test and reference Yes standard?

\begin{tabular}{ll} 
Did all patients receive the same reference standard? Yes \\
\hline
\end{tabular}

Were all patients included in the analysis?

Yes

Low

\section{Chew 2016}

\section{Study characteristics}

Patient sampling

Cross-sectional design, consecutive enrolment, retrospective data collection

Patient characteristics and setting

Presenting signs and symptoms: presumed pulmonary TB

Age: adults

Sex, female: not reported

HIV infection: not reported

History of TB: not reported

Sample size: 238

Clinical setting: inpatient

Laboratory level: central

Country: Singapore

World Bank Income Classification: high income

High TB burden country: no

High MDR-TB burden country: no

High TB/HIV burden country: no

Prevalence of TB cases in the study: $16.8 \%$

Index tests

Target condition and reference standard(s)
Index: Xpert MTB/RIF

Target condition: pulmonary TB

Reference standard for pulmonary TB: LJ and MGIT 960

Flow and timing

Comparative

Notes

Xpert MTB/RIF and Xpert MTB/RIF Ultra for pulmonary tuberculosis and rifampicin resistance in adults (Review) 
Chew 2016 (Continued)

Methodological quality

\begin{tabular}{lll}
\hline Item & $\begin{array}{l}\text { Authors' judge- } \\
\text { ment }\end{array}$ & $\begin{array}{c}\text { Risk of bias } \\
\text { Concerns }\end{array}$ \\
\hline DOMAIN 1: Patient Selection & Yes \\
\hline Was a consecutive or random sample of patients enrolled? & Yes & Yes \\
\hline Was a case-control design avoided? & & Low \\
\hline Did the study avoid inappropriate exclusions?
\end{tabular}

DOMAIN 2: Index Test Xpert MTB/RIF

Were the index test results interpreted without knowledge of the results of Yes the reference standard?

If a threshold was used, was it pre-specified? Yes

Low

Low

\section{DOMAIN 3: Reference Standard}

Is the reference standards likely to correctly classify the target condition? Yes

Were the reference standard results for TB detection interpreted without Yes

knowledge of the results of the index test?

Were the reference standard results for rifampicin resistance detection in-

terpreted without knowledge of the results of the index test?

\begin{tabular}{lll}
\hline & Low & Low \\
\hline DOMAIN 4: Flow and Timing & Yes \\
\hline $\begin{array}{l}\text { Was there an appropriate interval between index test and reference stan- } \\
\text { dard? }\end{array}$ & Yes \\
\hline Did all patients receive the same reference standard? & Yes \\
\hline Were all patients included in the analysis?
\end{tabular}

Low

\section{Chikaonda 2017}

\section{Study characteristics}

Patient sampling Cross-sectional design, random enrolment, prospective data collection 
Chikaonda 2017 (Continued)

\begin{tabular}{lll}
\hline & Low & Low \\
\hline
\end{tabular}

DOMAIN 3: Reference Standard

Is the reference standards likely to correctly classify the target condi- Yes tion?

Were the reference standard results for TB detection interpreted Unclear without knowledge of the results of the index test?

Were the reference standard results for rifampicin resistance detection interpreted without knowledge of the results of the index test?

\begin{tabular}{lll}
\hline DOMAIN 4: Flow and Timing & Unclear & Low \\
\hline $\begin{array}{l}\text { Was there an appropriate interval between index test and reference } \\
\text { standard? }\end{array}$ & Yes \\
\hline Did all patients receive the same reference standard? & Yes \\
\hline Were all patients included in the analysis? & \\
\hline
\end{tabular}

Low

\section{Cowan 2017}

\section{Study characteristics}

\section{Patient sampling}

Cross-sectional design, consecutive enrolment, both prospective and retrospective data collection

\section{Patient characteristics and setting}

Presenting signs and symptoms: presumed TB

Age: mean 50 years, range 18 - 88 years

Sex, female: $22 \%$

HIV infection: $24 \%$

History of TB: not reported

Sample size: 318

Clinical setting: inpatient

Laboratory level: central

Country: USA

World Bank Income Classification: high income

High TB burden country: no

High MDR-TB burden country: no 
Cowan 2017 (Continued)

High TB/HIV burden country: no

Prevalence of TB cases in the study: $6.3 \%$

\begin{tabular}{ll}
\hline Index tests & Index: Xpert MTB/RIF \\
\hline Target condition and reference standard(s) & Target condition: pulmonary TB \\
& Reference standard for pulmonary TB: 7H11 and MGIT 960 \\
& Target condition: rifampicin resistance \\
& Reference standard for rifampicin resistance: 7H11 and \\
& MGIT 960
\end{tabular}

Flow and timing

Comparative

Notes

\section{Methodological quality}

\begin{tabular}{|c|c|c|c|}
\hline Item & $\begin{array}{l}\text { Authors' judge- } \\
\text { ment }\end{array}$ & Risk of bias & $\begin{array}{l}\text { Applicability } \\
\text { concerns }\end{array}$ \\
\hline
\end{tabular}

DOMAIN 1: Patient Selection

\begin{tabular}{lcc}
\hline Was a consecutive or random sample of patients enrolled? & Yes & Yes \\
\hline Was a case-control design avoided? & Yes & Low \\
\hline Did the study avoid inappropriate exclusions? & High
\end{tabular}

\section{DOMAIN 2: Index Test Xpert MTB/RIF}

Were the index test results interpreted without knowledge of the re- Yes

sults of the reference standard?

If a threshold was used, was it pre-specified? Yes

\begin{tabular}{lll}
\hline & Low & Low \\
\hline DOMAIN 3: Reference Standard &
\end{tabular}

Is the reference standards likely to correctly classify the target condi- Yes tion?

Were the reference standard results for TB detection interpreted with- Yes out knowledge of the results of the index test?

Were the reference standard results for rifampicin resistance detection interpreted without knowledge of the results of the index test?

\begin{tabular}{llll} 
& Low & Low \\
\hline
\end{tabular}


Cowan 2017 (Continued)

DOMAIN 4: Flow and Timing

Was there an appropriate interval between index test and reference Yes standard?

Did all patients receive the same reference standard? Yes

Were all patients included in the analysis?

Yes

Low

\section{Davis 2014}

\section{Study characteristics}

Patient sampling

Cross-sectional design, consecutive enrolment, prospective data collection

Patient characteristics and setting

Presenting signs and symptoms: presumed pulmonary TB

Age: adults, median 52 years (IQR 39 to 60)

Sex, female: $35 \%$

HIV infection: $8 \%$

History of TB: yes, \% not reported

Sample size: 156

Clinical setting: inpatient

Laboratory level: central

Country: USA

World Bank Income Classification: high income

High TB burden country: no

High MDR-TB burden country: no

High TB/HIV burden country: no

Prevalence of TB cases in the study: $8.3 \%$

Index tests $\quad$ Index: Xpert MTB/RIF

Target condition and reference standard(s)

Target condition: pulmonary TB

Reference standard for pulmonary TB: LJ, 7H11, and MGIT 960

Target condition: rifampicin resistance

Reference standard for rifampicin resistance: $\mathrm{LJ}$ and $7 \mathrm{H} 11$ by proportion method and MGIT 960

Flow and timing

Of 227 eligible patients, 71 (31\%) were excluded because they were not tested 
Davis 2014 (Continued)

Comparative

$$
\text { Notes }
$$

\section{Methodological quality}

\begin{tabular}{|c|c|c|c|}
\hline Item & $\begin{array}{l}\text { Authors' judge- } \\
\text { ment }\end{array}$ & Risk of bias & $\begin{array}{l}\text { Applicability con- } \\
\text { cerns }\end{array}$ \\
\hline
\end{tabular}

\section{DOMAIN 1: Patient Selection}

\begin{tabular}{lcc}
\hline Was a consecutive or random sample of patients enrolled? & Yes & \\
\hline Was a case-control design avoided? & Yes & Yes \\
\hline Did the study avoid inappropriate exclusions? & Low & High
\end{tabular}

DOMAIN 2: Index Test Xpert MTB/RIF

Were the index test results interpreted without knowledge of the Yes results of the reference standard?

If a threshold was used, was it pre-specified?

Yes

\begin{tabular}{|c|c|c|}
\hline & Low & Low \\
\hline \multicolumn{3}{|l|}{ DOMAIN 3: Reference Standard } \\
\hline $\begin{array}{l}\text { Is the reference standards likely to correctly classify the target } \\
\text { condition? }\end{array}$ & Yes & \\
\hline $\begin{array}{l}\text { Were the reference standard results for TB detection interpreted } \\
\text { without knowledge of the results of the index test? }\end{array}$ & Yes & \\
\hline \multirow{2}{*}{$\begin{array}{l}\text { Were the reference standard results for rifampicin resistance de- } \\
\text { tection interpreted without knowledge of the results of the index } \\
\text { test? }\end{array}$} & Yes & \\
\hline & Low & Low \\
\hline \multicolumn{3}{|l|}{ DOMAIN 4: Flow and Timing } \\
\hline $\begin{array}{l}\text { Was there an appropriate interval between index test and refer- } \\
\text { ence standard? }\end{array}$ & Yes & \\
\hline Did all patients receive the same reference standard? & Yes & \\
\hline Were all patients included in the analysis? & No & \\
\hline
\end{tabular}

\section{High}


Dorman 2018

\section{Study characteristics}

Patient sampling

Cross-sectional design, consecutive enrolment, prospective data collection, multicentre study

Patient characteristics and setting

Presenting signs and symptoms: presumed pulmonary TB

Age: adults, median 28 years (IQR 28 to 50 )

Sex, female: $40 \%$

HIV infection: $44 \%$

History of TB: $21 \%$

Sample size: 1439 for detection of MTB, 551 for rifampicin resistance

Clinical setting: both outpatient and inpatient

Laboratory level: central (reference)

Country: Belarus, Brazil, China, Georgia, India, Kenya, South Africa, Uganda

World Bank Income Classification: low and middle income

High TB burden country: yes (Brazil, China, India, Kenya, South Africa)

High MDR-TB burden country: yes (Belarus, China, India, Kenya, South Africa)

High TB/HIV burden country: yes (Brazil, China, India, Kenya, South Africa, Uganda)

Prevalence of TB cases in the study: $32.1 \%$

Index tests Index: Xpert MTB/RIF and Xpert Ultra

Target condition and reference standard(s)

Target condition: pulmonary TB

Reference standard for pulmonary TB: LJ and MGIT 960

Target condition: rifampicin resistance

Reference standard for rifampicin resistance: MGIT 960

Flow and timing

Comparative

25 participants (3\%) who were smear-positive but in whom all cultures were negative were excluded from the analysis

\section{Methodological quality}

\begin{tabular}{llll}
\hline Item Authors' judgement & Risk of bias & $\begin{array}{l}\text { Applicability con- } \\
\text { cerns }\end{array}$
\end{tabular}

\section{DOMAIN 1: Patient Selection}

Was a consecutive or random sample of patients en- Yes rolled? 
Dorman 2018 (Continued)

Was a case-control design avoided? Yes

Did the study avoid inappropriate exclusions? Yes

\begin{tabular}{lcc}
\hline Low & Low \\
\hline
\end{tabular}

\section{DOMAIN 2: Index Test Xpert MTB/RIF}

Were the index test results interpreted without knowl- Yes

edge of the results of the reference standard?

If a threshold was used, was it pre-specified?

Yes

\begin{tabular}{lll}
\hline Low & Low \\
\hline
\end{tabular}

DOMAIN 3: Reference Standard

Is the reference standards likely to correctly classify Yes

the target condition?

Were the reference standard results for TB detection Yes

interpreted without knowledge of the results of the

index test?

Were the reference standard results for rifampicin resistance detection interpreted without knowledge of the results of the index test?

\begin{tabular}{lll}
\hline & Low Low \\
\hline
\end{tabular}

\section{DOMAIN 4: Flow and Timing}

Was there an appropriate interval between index test Yes and reference standard?

Did all patients receive the same reference standard? Yes

Were all patients included in the analysis?

No

\section{Unclear}

Friedrich 2011

\section{Study characteristics}

Patient sampling

Patient characteristics and setting
Cross-sectional design, consecutive enrolment, prospective data collection

Presenting signs and symptoms: people recently diagnosed with smear-positive first time TB, untreated

Age: 18 to 65 years

Sex, female: not reported

HIV infection: not reported 
Friedrich 2011 (Continued)

History of TB: not reported

Sample size: 126

Clinical setting: smear examination at TB clinic and referred to inpatient settings

Laboratory level: central

Country: South Africa, Cape Town

World Bank Income Classification: middle income

High TB burden country: yes

High MDR-TB burden country: yes

High TB/HIV burden country: yes

TB incidence rate: 993 per 100,000

MDR-TB prevalence: $\%$ MDR-TB among new TB cases $=0.9 \%$ (Source: survey in Western Cape Province, 2002) and among retreatment cases $=4.0 \%$ (Source: survey in Western Cape Province, 2002)

Prevalence of TB cases in the study: $100.0 \%$

\begin{tabular}{ll}
\hline Index tests & Index: Xpert MTB/RIF \\
\hline Target condition and reference standard(s) & Target condition: pulmonary TB \\
& Reference standard for pulmonary TB: MGIT 960 \\
& Target condition: rifampicin resistance \\
& Reference standard for rifampicin resistance: MGIT 960
\end{tabular}

Flow and timing

Comparative

\section{Notes}

The aim of this study was to assess NAATs for selecting participants for clinical trials of anti-TB medication. People with severe co-morbidities were excluded. This study was used only for determination of sensitivity because all enrolled participants were predetermined to have TB disease

\section{Methodological quality}

\begin{tabular}{llll}
\hline Item & Authors' judgement & Risk of bias & Applicability concerns \\
\hline DOMAIN 1: Patient Selection & & \\
\hline $\begin{array}{l}\text { Was a consecutive or random sample of patients } \\
\text { enrolled? }\end{array}$ & Yes & \\
\hline Was a case-control design avoided? & Yes & High & Low \\
\hline Did the study avoid inappropriate exclusions? & No & & Ho
\end{tabular}


Friedrich 2011 (Continued)

\section{DOMAIN 2: Index Test Xpert MTB/RIF}

Were the index test results interpreted without knowledge of the results of the reference standard?

If a threshold was used, was it pre-specified? Yes
Low
Unclear

\section{DOMAIN 3: Reference Standard}

Is the reference standards likely to correctly clas- Yes sify the target condition?

Were the reference standard results for TB detec- Yes tion interpreted without knowledge of the results of the index test?

Were the reference standard results for rifampicin Yes resistance detection interpreted without knowledge of the results of the index test?

\begin{tabular}{lll}
\hline Low & Low \\
\hline
\end{tabular}

\section{DOMAIN 4: Flow and Timing}

Was there an appropriate interval between index Yes test and reference standard?

Did all patients receive the same reference stan- Yes dard?

Were all patients included in the analysis? Yes

Low

\section{Geleta 2015}

\section{Study characteristics}

\section{Patient sampling}

\section{Patient characteristics and setting}

Cross-sectional design, consecutive enrolment, direction of data collection unclear

Presenting signs and symptoms: signs, symptoms, or chest $x$-ray suggestive of TB

Age: median 35 years, range 18 to 82 years

Sex, female: $37 \%$

HIV infection: not reported

History of TB: not reported

Sample size: 220 
Geleta 2015 (Continued)

Clinical setting: not reported

Laboratory level: central

Country: Ethiopia

World Bank Income Classification: low income

High TB burden country: yes

High MDR-TB burden country: yes

High TB/HIV burden country: yes

Prevalence of TB cases in the study: $26.4 \%$

\begin{tabular}{ll}
\hline Index tests & Index: Xpert MTB/RIF \\
\hline Target condition and reference standard(s) & Target condition: pulmonary TB \\
& Reference standard for pulmonary TB: LJ and MGIT 960 \\
\hline
\end{tabular}

Flow and timing

Comparative

Notes

\section{Methodological quality}

\begin{tabular}{lll}
\hline Item & $\begin{array}{l}\text { Authors' judge- } \\
\text { ment }\end{array}$ & $\begin{array}{l}\text { Risk of bias } \\
\begin{array}{l}\text { Applicability } \\
\text { concerns }\end{array}\end{array}$ \\
\hline
\end{tabular}

\section{DOMAIN 1: Patient Selection}

\begin{tabular}{lcc}
\hline Was a consecutive or random sample of patients enrolled? & Yes & Yes \\
\hline Was a case-control design avoided? & Yes & Low
\end{tabular}

\section{DOMAIN 2: Index Test Xpert MTB/RIF}

Were the index test results interpreted without knowledge of the results Yes of the reference standard?

\begin{tabular}{lcc}
\hline If a threshold was used, was it pre-specified? & Yes \\
\hline & Low Low \\
\hline
\end{tabular}

\section{DOMAIN 3: Reference Standard}

Is the reference standards likely to correctly classify the target condi- Yes tion?

Were the reference standard results for TB detection interpreted with- Yes out knowledge of the results of the index test? 
Geleta 2015 (Continued)

Were the reference standard results for rifampicin resistance detection interpreted without knowledge of the results of the index test?

Low

Low

\section{DOMAIN 4: Flow and Timing}

Was there an appropriate interval between index test and reference

Yes

standard?

\begin{tabular}{ll}
\hline Did all patients receive the same reference standard? Yes \\
\hline
\end{tabular}

Were all patients included in the analysis? Yes

\section{Low}

\section{Hanif 2011}

\section{Study characteristics}

Patient sampling
Patient characteristics and setting

Cross-sectional design, consecutive enrolment, prospective data collection

Presenting signs and symptoms: presumed TB based on presence of cough and radiographic findings

Age: range 20 to 57 years

Sex, female: not reported

HIV infection: not reported

History of TB: not reported

Sample size: 206

Clinical setting: laboratory-based

Laboratory level: central

Country: Kuwait

World Bank Income Classification: high income

High TB burden country: no

High MDR-TB burden country: no

High TB/HIV burden country: no

TB incidence rate: 36 per 100,000

MDR-TB prevalence: $\%$ MDR-TB among new TB cases $=0 \%$ and among retreatment cases $=12 \%$ (Source: nationwide surveillance, 2011)

Prevalence of TB cases in the study: $29.1 \%$ 
Hanif 2011 (Continued)

Target condition: rifampicin resistance

Reference standard for rifampicin resistance: BACTEC 460

Flow and timing

Comparative

Notes No participants were found to have rifampicin resistance

Methodological quality

\begin{tabular}{|c|c|c|c|}
\hline Item & Authors' judgement & Risk of bias & $\begin{array}{l}\text { Applicability con- } \\
\text { cerns }\end{array}$ \\
\hline
\end{tabular}

\section{DOMAIN 1: Patient Selection}

\begin{tabular}{llll}
\hline Was a consecutive or random sample of patients enrolled? & Yes \\
\hline Was a case-control design avoided? & Yes & \\
\hline Did the study avoid inappropriate exclusions? & Yes & Low & Unclear \\
\hline
\end{tabular}

DOMAIN 2: Index Test Xpert MTB/RIF

Were the index test results interpreted without knowledge Yes of the results of the reference standard?

If a threshold was used, was it pre-specified? Yes

\begin{tabular}{l} 
Low Lw L L L L L L \\
\hline
\end{tabular}

\section{DOMAIN 3: Reference Standard}

Is the reference standards likely to correctly classify the tar- Yes get condition?

Were the reference standard results for TB detection inter- No preted without knowledge of the results of the index test?

Were the reference standard results for rifampicin resis- Yes tance detection interpreted without knowledge of the results of the index test?

\begin{tabular}{lll}
\hline & High & Low \\
\hline DOMAIN 4: Flow and Timing & \\
\hline $\begin{array}{l}\text { Was there an appropriate interval between index test and } \\
\text { reference standard? }\end{array}$ & Yes \\
\hline Did all patients receive the same reference standard? & Yes \\
\hline Were all patients included in the analysis? &
\end{tabular}


Hanrahan 2013

\section{Study characteristics}

Patient sampling

Cross-sectional design, consecutive enrolment, prospective data collection

\section{Patient characteristics and setting}

Presenting signs and symptoms: prolonged ( $>2$ weeks) cough and/or other TB symptoms

Age: 18 years and older, median 35 years (IQR 29 to 44)

Sex, female: $65 \%$

HIV infection: $69 \%$

History of TB: $10 \%$

Sample size: 553

Clinical setting: outpatient

Laboratory level: peripheral

Country: South Africa, Johannesburg

World Bank Income Classification: middle income

High TB burden country: yes

High MDR-TB burden country: yes

High TB/HIV burden country: yes

TB incidence rate: 993 per 100,000

MDR-TB prevalence: \% MDR-TB among new TB cases $=1.4 \%$

(Source: survey in Gauteng province, 2002) and among retreatment cases $=5.5 \%$ (Source: survey in Gauteng province, 2002)

Prevalence of TB cases in the study: $11.6 \%$

Index tests Index: Xpert MTB/RIF

Target condition and reference standard(s)

Target condition: pulmonary TB

Reference standard for pulmonary TB: MGIT 960

Flow and timing

Comparative

\section{Notes}

\section{Methodological quality}


Hanrahan 2013 (Continued)

Item

Authors' judgement Risk of bias

Applicability con-

cerns

DOMAIN 1: Patient Selection

Was a consecutive or random sample of patients enrolled? Y

\begin{tabular}{ll}
\hline Was a case-control design avoided? Yes \\
\hline
\end{tabular}

Did the study avoid inappropriate exclusions? Yes

\begin{tabular}{llcl}
\hline DOMAIN 2: Index Test Xpert MTB/RIF & Low & Low \\
\hline $\begin{array}{l}\text { Were the index test results interpreted without knowledge of } \\
\text { the results of the reference standard? }\end{array}$ & Yes & Low & Low \\
\hline If a threshold was used, was it pre-specified? & & Yow
\end{tabular}

\section{DOMAIN 3: Reference Standard}

Is the reference standards likely to correctly classify the target Yes condition?

Were the reference standard results for TB detection interpret- Yes ed without knowledge of the results of the index test?

Were the reference standard results for rifampicin resistance detection interpreted without knowledge of the results of the index test?

\begin{tabular}{lll}
\hline LOMAIN 4: Flow and Timing & Low \\
\hline $\begin{array}{l}\text { Was there an appropriate interval between index test and refer- } \\
\text { ence standard? }\end{array}$ & Yes \\
\hline Did all patients receive the same reference standard? & Yes \\
\hline Were all patients included in the analysis? & & Low \\
\hline
\end{tabular}

Hanrahan 2014

\section{Study characteristics}

Patient sampling

Cross-sectional design, consecutive enrolment, retrospective data collection

Patient characteristics and setting

Presenting signs and symptoms: presumed pulmonary TB

Xpert MTB/RIF and Xpert MTB/RIF Ultra for pulmonary tuberculosis and rifampicin resistance in adults (Review)

Copyright (C 2019 The Authors. Cochrane Database of Systematic Reviews published by John Wiley \& Sons, Ltd. on behalf of The Cochrane

Collaboration. 
Sex, female: $62 \%$

HIV infection: $58 \%$

History of TB: not reported

Sample size: 2082

Clinical setting: outpatient

Laboratory level: central

Country: South Africa

World Bank Income Classification: middle income

High TB burden country: yes

High MDR-TB burden country: yes

High TB/HIV burden country: yes

Prevalence of TB cases in the study: $19.5 \%$

\begin{tabular}{ll}
\hline Index tests $\quad$ Index: Xpert MTB/RIF &
\end{tabular}

Target condition and reference standard(s)

Target condition: Pulmonary TB

Reference standard for pulmonary TB: MGIT 960

Flow and timing

Comparative

Notes

This study focused on drug-susceptible TB and therefore excluded 10 people found to have rifampicin resistance on Xpert

\section{Methodological quality}

\begin{tabular}{lll}
\hline Item & $\begin{array}{l}\text { Authors' judge- } \\
\text { ment }\end{array}$ & $\begin{array}{l}\text { Risk of bias } \\
\text { Applicability con- } \\
\text { cerns }\end{array}$ \\
\hline
\end{tabular}

\section{DOMAIN 1: Patient Selection}

\begin{tabular}{lcc}
\hline Was a consecutive or random sample of patients enrolled? & Yes \\
\hline Was a case-control design avoided? & Yes & Yes \\
\hline Did the study avoid inappropriate exclusions? & Low Low
\end{tabular}

\section{DOMAIN 2: Index Test Xpert MTB/RIF}

Were the index test results interpreted without knowledge of the re- Yes sults of the reference standard? 
Hanrahan 2014 (Continued)

Low

\section{DOMAIN 3: Reference Standard}

Is the reference standards likely to correctly classify the target con- Yes dition?

Were the reference standard results for TB detection interpreted Yes without knowledge of the results of the index test?

Were the reference standard results for rifampicin resistance detec-

tion interpreted without knowledge of the results of the index test?

\begin{tabular}{lll}
\hline LOMAIN 4: Flow and Timing & Low & \\
\hline $\begin{array}{l}\text { Was there an appropriate interval between index test and reference } \\
\text { standard? }\end{array}$ & Yes Yes \\
\hline Did all patients receive the same reference standard? & Unclear & Unclear \\
\hline Were all patients included in the analysis? & &
\end{tabular}

\section{Helb 2010}

\section{Study characteristics}

Patient sampling

Cross-sectional design, consecutive enrolment, retrospective data collection

Patient characteristics and setting

Presenting signs and symptoms: cough lasting at least 2 weeks

Age: median 34 years, range 18 to 76 years

Sex, female: $30.8 \%$

HIV infection: $0.9 \%$

History of TB: $1.9 \%$

Sample size: 107

Clinical setting: TB hospital, unclear whether inpatient or outpatient or both

Laboratory level: central

Country: Vietnam

World Bank Income Classification: middle income

High TB burden country: yes

High MDR-TB burden country: yes

High TB/HIV burden country: no 
Helb 2010 (Continued)

TB incidence rate: 199 per 100,000

MDR-TB prevalence: Percent MDR-TB among new TB cases $=2.7 \%$

(Source: nationwide survey, 2006) and among retreatment cases =

19\% (Source: nationwide survey, 2006)

Proportion of TB cases in the study: $76.6 \%$

Index tests

Target condition and reference standard(s)
Index: Xpert MTB/RIF assay

Target condition: pulmonary TB

Reference standard: LJ culture and MGIT 960

Flow and timing

Comparative

$\begin{array}{ll}\text { Notes } & \text { Rifampicin resistance data were not reported }\end{array}$

Methodological quality

\begin{tabular}{llll}
\hline Item Authors' judgement & Risk of bias & \multicolumn{1}{l}{$\begin{array}{l}\text { Applicability con- } \\
\text { cerns }\end{array}$}
\end{tabular}

\section{DOMAIN 1: Patient Selection}

\begin{tabular}{llll}
\hline Was a consecutive or random sample of patients enrolled? & Yes \\
\hline Was a case-control design avoided? & Yes & \\
\hline Did the study avoid inappropriate exclusions? & Yes & Low & Unclear \\
\hline
\end{tabular}

\section{DOMAIN 2: Index Test Xpert MTB/RIF}

Were the index test results interpreted without knowledge of Yes

the results of the reference standard?

\begin{tabular}{lll}
\hline If a threshold was used, was it pre-specified? & Yes & Low Lw L L L L \\
\hline
\end{tabular}

\section{DOMAIN 3: Reference Standard}

Is the reference standards likely to correctly classify the target Yes condition?

Were the reference standard results for TB detection inter- Yes preted without knowledge of the results of the index test?

Were the reference standard results for rifampicin resistance detection interpreted without knowledge of the results of the index test?

\begin{tabular}{l} 
Low L L L L \\
\hline
\end{tabular}


Helb 2010 (Continued)

\section{DOMAIN 4: Flow and Timing}

Was there an appropriate interval between index test and ref- Yes erence standard?

Did all patients receive the same reference standard? Yes

Were all patients included in the analysis? Unclear

\section{Unclear}

\section{Henostroza 2016}

\section{Study characteristics}

\begin{tabular}{|c|c|}
\hline Patient sampling & $\begin{array}{l}\text { Cross-sectional design, consecutive enrolment, prospective } \\
\text { data collection }\end{array}$ \\
\hline \multirow[t]{14}{*}{ Patient characteristics and setting } & $\begin{array}{l}\text { Presenting signs and symptoms: ART-naïve people presenting } \\
\text { for initiation of HIV care }\end{array}$ \\
\hline & Age: 16 years and older, median 34 years (IQR 29 to 40) \\
\hline & Sex, female: $49 \%$ \\
\hline & HIV infection: $100 \%$ \\
\hline & History of TB: not reported \\
\hline & Sample size: 332 \\
\hline & Clinical setting: outpatient \\
\hline & Laboratory level: central \\
\hline & Country: Zambia \\
\hline & World Bank Income Classification: middle income \\
\hline & High TB burden country: yes \\
\hline & High MDR-TB burden country: no \\
\hline & High TB/HIV burden country: yes \\
\hline & Prevalence of TB cases in the study: $18.6 \%$ \\
\hline
\end{tabular}

\begin{tabular}{ll}
\hline Index tests $\quad$ Index: Xpert MTB/RIF \\
\hline
\end{tabular}

Target condition and reference standard(s)

Target condition: pulmonary TB

Reference standard for pulmonary TB: LJ and MGIT 960

\section{Flow and timing}

Comparative

Notes

The paper states that outpatients in this cohort were likely to have been less ill than hospitalized patients 
Henostroza 2016 (Continued)

Methodological quality

\begin{tabular}{lll}
\hline Item & $\begin{array}{l}\text { Authors' judge- } \\
\text { ment }\end{array}$ & $\begin{array}{l}\text { Risk of bias } \\
\text { Applicability con- } \\
\text { cerns }\end{array}$ \\
\hline
\end{tabular}

\section{DOMAIN 1: Patient Selection}

\begin{tabular}{lcc}
\hline Was a consecutive or random sample of patients enrolled? & Yes & \\
\hline Was a case-control design avoided? & Yes & Yes \\
\hline Did the study avoid inappropriate exclusions? & Low Low \\
\hline
\end{tabular}

DOMAIN 2: Index Test Xpert MTB/RIF

Were the index test results interpreted without knowledge of the re- Yes sults of the reference standard?

If a threshold was used, was it pre-specified? Yes

\begin{tabular}{llll}
\hline Low & Low \\
\hline
\end{tabular}

DOMAIN 3: Reference Standard

Is the reference standards likely to correctly classify the target con- Yes dition?

Were the reference standard results for TB detection interpreted Yes without knowledge of the results of the index test?

Were the reference standard results for rifampicin resistance detection interpreted without knowledge of the results of the index test?

\begin{tabular}{lll}
\hline & Low & Low \\
\hline DOMAIN 4: Flow and Timing & & \\
\hline $\begin{array}{l}\text { Was there an appropriate interval between index test and reference } \\
\text { standard? }\end{array}$ & Yes & Yes \\
\hline Did all patients receive the same reference standard? & & Low \\
\hline Were all patients included in the analysis? & & \\
\hline
\end{tabular}

Huang 2015

\section{Study characteristics}

Patient sampling

Cross-sectional design, manner of enrolment unclear, prospective data collection 
Huang 2015 (Continued)

Patient characteristics and setting
Presenting signs and symptoms: not reported

Age: mean 42 years, range 15 to 55 years

Sex, female: $44 \%$

HIV infection: not reported

History of TB: not reported

Sample size: 378

Clinical setting: laboratory-based

Laboratory level: peripheral

Country: China

World Bank Income Classification: middle income

High TB burden country: yes

High MDR-TB burden country: yes

High TB/HIV burden country: yes

Prevalence of TB cases in the study: $49.7 \%$

Index: Xpert MTB/RIF

Target condition: pulmonary TB

Reference standard for pulmonary TB: MGIT 960

Target condition: Rifampicin resistance

Reference standard for rifampicin resistance: MGIT 960

Flow and timing

Comparative

Notes

Methodological quality

\begin{tabular}{|c|c|c|c|}
\hline Item & $\begin{array}{l}\text { Authors' judge- } \\
\text { ment }\end{array}$ & Risk of bias & $\begin{array}{l}\text { Applicability } \\
\text { concerns }\end{array}$ \\
\hline \multicolumn{4}{|l|}{ DOMAIN 1: Patient Selection } \\
\hline Was a consecutive or random sample of patients enrolled? & Unclear & & \\
\hline Was a case-control design avoided? & Yes & & \\
\hline \multirow[t]{2}{*}{ Did the study avoid inappropriate exclusions? } & Yes & & \\
\hline & & Unclear & Low \\
\hline
\end{tabular}

DOMAIN 2: Index Test Xpert MTB/RIF 
Huang 2015 (Continued)

Were the index test results interpreted without knowledge of the re- Yes sults of the reference standard?

If a threshold was used, was it pre-specified?

Yes

\begin{tabular}{llll}
\hline & Low & Low \\
\hline
\end{tabular}

\section{DOMAIN 3: Reference Standard}

Is the reference standards likely to correctly classify the target condi- Yes tion?

Were the reference standard results for TB detection interpreted without knowledge of the results of the index test?

Were the reference standard results for rifampicin resistance detecYes tion interpreted without knowledge of the results of the index test?

\begin{tabular}{lll}
\hline & Low & Low \\
\hline DOMAIN 4: Flow and Timing & & \\
\hline $\begin{array}{l}\text { Was there an appropriate interval between index test and reference } \\
\text { standard? }\end{array}$ & Yes \\
\hline Did all patients receive the same reference standard? & Yes \\
\hline Were all patients included in the analysis?
\end{tabular}

Low

Huh 2014

\section{Study characteristics}

Patient sampling

Patient characteristics and setting
Cross-sectional design, consecutive enrolment, retrospective data collection

Presenting signs and symptoms: presumptive pulmonary TB as defined by the presence of the clinical symptoms (cough, fever, night sweats, or weight loss) and radiologic findings compatible with TB, in either a chest $x$-ray or a computed tomography scan

Age: median 58 years, range 18 to 93 years

Sex, female: $34 \%$

HIV infection: $0.3 \%$

History of TB: not reported

Sample size: 271

Clinical setting: tertiary care hospital, unclear if outpatient, inpatient, or both

Laboratory level: central 
Huh 2014 (Continued)

Country: Republic of Korea

World Bank Income Classification: high income

High TB burden country: no

High MDR-TB burden country: no

High TB/HIV burden country: no

Prevalence of TB cases in the study: $38.4 \%$

\begin{tabular}{|c|c|c|c|}
\hline Index tests & \multicolumn{3}{|l|}{ Index: Xpert MTB/RIF } \\
\hline \multirow[t]{4}{*}{ Target condition and reference standard(s) } & \multicolumn{3}{|c|}{ Target condition: pulmonary TB } \\
\hline & \multicolumn{3}{|c|}{ Reference standard for pulmonary TB: MGIT 960, Ogawa culture } \\
\hline & \multicolumn{3}{|c|}{ Target condition: rifampicin resistance } \\
\hline & \multicolumn{3}{|c|}{ Reference standard for rifampicin resistance: MGIT 960, LJ-DST } \\
\hline \multicolumn{4}{|l|}{ Flow and timing } \\
\hline \multicolumn{4}{|l|}{ Comparative } \\
\hline \multicolumn{4}{|l|}{ Notes } \\
\hline \multicolumn{4}{|l|}{ Methodological quality } \\
\hline Item & Authors' judgement & Risk of bias & $\begin{array}{l}\text { Applicability con- } \\
\text { cerns }\end{array}$ \\
\hline
\end{tabular}

\section{DOMAIN 1: Patient Selection}

\begin{tabular}{lll}
\hline Was a consecutive or random sample of patients enrolled? & Yes \\
\hline Was a case-control design avoided? & Yes & \\
\hline Did the study avoid inappropriate exclusions? & Yes & Low \\
\hline
\end{tabular}

\section{DOMAIN 2: Index Test Xpert MTB/RIF}

Were the index test results interpreted without knowledge Yes

of the results of the reference standard?

\begin{tabular}{llc}
\hline If a threshold was used, was it pre-specified? & Yes & Low Low \\
\hline
\end{tabular}

\section{DOMAIN 3: Reference Standard}

Is the reference standards likely to correctly classify the tar- Yes get condition?

Were the reference standard results for TB detection inter- $\quad$ Yes preted without knowledge of the results of the index test? 
Huh 2014 (Continued)

Were the reference standard results for rifampicin resistance detection interpreted without knowledge of the results of the index test?

Low

Low

\section{DOMAIN 4: Flow and Timing}

Was there an appropriate interval between index test and Yes reference standard?

Did all patients receive the same reference standard? Yes

Were all patients included in the analysis? Yes

Low

Jo 2016

\section{Study characteristics}

Patient sampling

Cross-sectional design, consecutive enrolment, retrospective data collection

Patient characteristics and setting

Presenting signs and symptoms: not reported, included patients from bronchoscopy registry, primarily smear-negative.

Age: adults, mean 63 years (SD 17)

Sex, female: $34 \%$

HIV infection: $0.3 \%$

History of TB: $15 \%$

Sample size: 320

Clinical setting: not reported

Laboratory level: central

Country: Republic of Korea

World Bank Income Classification: high income

High TB burden country: no

High MDR-TB burden country: no

High TB/HIV burden country: no

Prevalence of TB cases in the study: $20.0 \%$

Index tests Index: Xpert MTB/RIF

Target condition and reference standard(s)

Target condition: pulmonary TB

Reference standard for pulmonary TB: Ogawa and MGIT 960 
Jo 2016 (Continued)

Flow and timing

\section{Comparative}

Notes

Only 10 bronchoscopically obtained specimens (7.69\%) were smear-positive

\section{Methodological quality}

\begin{tabular}{|c|c|c|c|}
\hline Item & $\begin{array}{l}\text { Authors' judge- } \\
\text { ment }\end{array}$ & Risk of bias & $\begin{array}{l}\text { Applicability con- } \\
\text { cerns }\end{array}$ \\
\hline
\end{tabular}

\section{DOMAIN 1: Patient Selection}

\begin{tabular}{lcc}
\hline Was a consecutive or random sample of patients enrolled? & Yes & \\
\hline Was a case-control design avoided? & Yes & \\
\hline Did the study avoid inappropriate exclusions? & No & High \\
\hline
\end{tabular}

\section{DOMAIN 2: Index Test Xpert MTB/RIF}

Were the index test results interpreted without knowledge of the re- Yes sults of the reference standard?

\begin{tabular}{lcc}
\hline If a threshold was used, was it pre-specified? & Yes \\
\hline & Low Low \\
\hline
\end{tabular}

\section{DOMAIN 3: Reference Standard}

Is the reference standards likely to correctly classify the target con- Yes dition?

Were the reference standard results for TB detection interpreted Yes without knowledge of the results of the index test?

Were the reference standard results for rifampicin resistance detec-

tion interpreted without knowledge of the results of the index test?

\begin{tabular}{lll}
\hline & Low & Low \\
\hline DOMAIN 4: Flow and Timing & Yes \\
\hline $\begin{array}{l}\text { Was there an appropriate interval between index test and reference } \\
\text { standard? }\end{array}$ & Yes \\
\hline Did all patients receive the same reference standard? & & Low \\
\hline Were all patients included in the analysis? &
\end{tabular}


Kawkitinarong 2017

\section{Study characteristics}

\section{Patient sampling}

Cross-sectional design, random enrolment for 2 sites, consecutive enrolment for 1 site, prospective data collection

\section{Patient characteristics and setting}

Presenting signs and symptoms: presumed pulmonary TB

Age: adults, median 41 years (IQR 30.8 to 54.3 )

Sex, female: $42.5 \%$

HIV infection: $25.9 \%$

History of TB: not reported

Sample size: 389

Clinical setting: not reported

Laboratory level: central

Country: Thailand

World Bank Income Classification: middle income

High TB burden country: yes

High MDR-TB burden country: yes

High TB/HIV burden country: yes

Prevalence of TB cases in the study: $64.3 \%$ Index: Xpert MTB/RIF

Target condition and reference standard(s)

Target condition: Pulmonary TB

Reference standard for pulmonary TB: Ogawa and MGIT 960

Target condition: Rifampicin resistance

Reference standard for rifampicin resistance: MGIT 960

Flow and timing

Comparative

\section{Notes}

\section{Methodological quality}

\begin{tabular}{|c|c|c|c|}
\hline Item & $\begin{array}{l}\text { Authors' judge- } \\
\text { ment }\end{array}$ & Risk of bias & $\begin{array}{l}\text { Applicability con- } \\
\text { cerns }\end{array}$ \\
\hline
\end{tabular}

DOMAIN 1: Patient Selection

Was a consecutive or random sample of patients enrolled? Yes

Was a case-control design avoided?

Yes 
Kawkitinarong 2017 (Continued)

Did the study avoid inappropriate exclusions?

Yes

\begin{tabular}{lll}
\hline & Low & Unclear \\
\hline
\end{tabular}

DOMAIN 2: Index Test Xpert MTB/RIF

Were the index test results interpreted without knowledge of the re- Yes sults of the reference standard?

If a threshold was used, was it pre-specified? Yes

\begin{tabular}{lll}
\hline Low & Low \\
\hline
\end{tabular}

\section{DOMAIN 3: Reference Standard}

Is the reference standards likely to correctly classify the target con- Yes dition?

Were the reference standard results for TB detection interpreted Yes without knowledge of the results of the index test?

Were the reference standard results for rifampicin resistance detec- Yes tion interpreted without knowledge of the results of the index test?

Low

DOMAIN 4: Flow and Timing

Was there an appropriate interval between index test and reference Yes standard?

\begin{tabular}{ll} 
Did all patients receive the same reference standard? Yes \\
\hline
\end{tabular}

Were all patients included in the analysis?

Yes

Low

\section{Kim CH 2015}

\section{Study characteristics}

Patient sampling

Cross-sectional design, manner of participant selection unknown, retrospective data collection

Patient characteristics and setting

Presenting signs and symptoms: presumed TB

Age: mean 56 years (SD 18)

Sex, female: $43 \%$

HIV infection: $0.1 \%$

History of TB: not reported

Sample size: 405

Clinical setting: inpatient 
Kim CH 2015 (Continued)

\author{
Laboratory level: central \\ Country: Republic of Korea \\ World Bank Income Classification: high income \\ High TB burden country: no \\ High MDR-TB burden country: no \\ High TB/HIV burden country: no \\ Prevalence of TB cases in the study: $12.8 \%$
}

Index tests

Target condition and reference standard(s)
Index: Xpert MTB/RIF

Target condition: pulmonary TB

Reference standard for pulmonary TB: Ogawa and MGIT 960

Target condition: rifampicin resistance

Reference standard for rifampicin resistance: LJ, concentration method

Flow and timing

Comparative

Notes

Methodological quality

\begin{tabular}{llll}
\hline Item & $\begin{array}{l}\text { Authors' judge- } \\
\text { ment }\end{array}$ & $\begin{array}{l}\text { Risk of bias } \\
\text { concerns }\end{array}$ \\
\hline
\end{tabular}

\title{
DOMAIN 1: Patient Selection
}

\begin{tabular}{lccl}
\hline Was a consecutive or random sample of patients enrolled? & Unclear & \\
\hline Was a case-control design avoided? & Yes & Yes & Unclear High \\
\hline Did the study avoid inappropriate exclusions? & & \\
\hline
\end{tabular}

Were the index test results interpreted without knowledge of the re- Yes sults of the reference standard?

\begin{tabular}{lcc}
\hline If a threshold was used, was it pre-specified? & Yes & Low \\
\hline
\end{tabular}

\section{DOMAIN 3: Reference Standard}

Is the reference standards likely to correctly classify the target condi- Yes tion? 
Kim CH 2015 (Continued)

Were the reference standard results for TB detection interpreted without knowledge of the results of the index test?

Were the reference standard results for rifampicin resistance detection interpreted without knowledge of the results of the index test?
Yes

Unclear

\begin{tabular}{lll}
\hline & Low & Low \\
\hline DOMAIN 4: Flow and Timing & Yes \\
\hline $\begin{array}{l}\text { Was there an appropriate interval between index test and reference } \\
\text { standard? }\end{array}$ & Yes Yes \\
\hline Did all patients receive the same reference standard? & Low \\
\hline Were all patients included in the analysis? &
\end{tabular}

\section{Ko 2016}

\section{Study characteristics}

Patient sampling

Patient characteristics and setting
Cross-sectional design, consecutive enrolment, retrospective data collection

Presenting signs and symptoms: presumed pulmonary TB

Age: adults (range 17 - 87 years), median 58 years (IQR 43 to 71 )

Sex, female: $42 \%$

HIV infection: $0.4 \%$

History of TB: not reported

Sample size: 249

Clinical setting: not reported

Laboratory level: central

Country: Republic of Korea

World Bank Income Classification: high income

High TB burden country: no

High MDR-TB burden country: no

High TB/HIV burden country: no

Prevalence of TB cases in the study: $42.2 \%$ Index: Xpert MTB/RIF
Target condition: Pulmonary TB 
Ko 2016 (Continued)

Flow and timing

Comparative

Notes

\section{Methodological quality}

\begin{tabular}{|c|c|c|c|}
\hline Item & $\begin{array}{l}\text { Authors' judge- } \\
\text { ment }\end{array}$ & Risk of bias & $\begin{array}{l}\text { Applicability } \\
\text { concerns }\end{array}$ \\
\hline
\end{tabular}

\section{DOMAIN 1: Patient Selection}

\begin{tabular}{lc}
\hline Was a consecutive or random sample of patients enrolled? & Yes \\
\hline Was a case-control design avoided? & Yes \\
\hline Did the study avoid inappropriate exclusions? & Yes \\
\hline
\end{tabular}

\begin{tabular}{lll}
\hline & Low & Unclear \\
\hline DOMAIN 2: Index Test Xpert MTB/RIF &
\end{tabular}

\section{DOMAIN 2: Index Test Xpert MTB/RIF}

Were the index test results interpreted without knowledge of the results Yes of the reference standard?

\begin{tabular}{lll}
\hline If a threshold was used, was it pre-specified? & Yes & Low Low \\
\hline
\end{tabular}

\section{DOMAIN 3: Reference Standard}

Is the reference standards likely to correctly classify the target condi- Yes tion?

Were the reference standard results for TB detection interpreted with- Unclear out knowledge of the results of the index test?

Were the reference standard results for rifampicin resistance detection interpreted without knowledge of the results of the index test?

\begin{tabular}{lcc} 
& Unclear & Low \\
\hline DOMAIN 4: Flow and Timing & Yes \\
\hline $\begin{array}{l}\text { Was there an appropriate interval between index test and reference } \\
\text { standard? }\end{array}$ & Yes \\
\hline Did all patients receive the same reference standard? & Yes \\
\hline Were all patients included in the analysis? &
\end{tabular}


Kurbaniyazova 2017

\section{Study characteristics}

Patient sampling

Cross-sectional design, consecutive enrolment, retrospective data collection

Patient characteristics and setting

Presenting signs and symptoms: people with cough of 2 weeks, fever, night sweats and weight loss; TB patients with positive smear results or a sputum smear-negative result but radiographic abnormalities suggestive of TB; retreatment cases; contacts of TB or MDR-TB patients; patients with severe clinical condition; and HIV-positive patients or those with unknown HIV status in high-risk settings such as migrants or prisoners; according to the diagnostic algorithm of Kyrgyzstan's National Tuberculosis Programme's clinical protocol

Age: adults $>18$

Sex, female: not reported

HIV infection: not reported

History of TB: not reported

Sample size: 2734

Clinical setting: outpatient

Laboratory level: central

Country: Kyrgyzstan

World Bank Income Classification: middle income

High TB burden country: no

High MDR-TB burden country: yes

High TB/HIV burden country: no

Prevalence of TB cases in the study: $62.2 \%$

Index tests Index: Xpert MTB/RIF

Target condition and reference standard(s)

Target condition: pulmonary TB

Reference standard for pulmonary TB: LJ and MGIT

Target condition: Rifampicin resistance

Reference standard for rifampicin resistance: LJ and MGIT

\section{Flow and timing}

Comparative

Notes

\section{Methodological quality}

Item Authors' judgement $\quad$ Risk of bias $\quad \begin{aligned} & \text { Applicability con- } \\ & \text { cerns }\end{aligned}$


Kurbaniyazova 2017 (Continued)

DOMAIN 1: Patient Selection

Was a consecutive or random sample of patients en- Yes rolled?

\begin{tabular}{llll}
\hline Was a case-control design avoided? & Yes & \\
\hline Did the study avoid inappropriate exclusions? & Yes & Low & Low \\
\hline
\end{tabular}

\section{DOMAIN 2: Index Test Xpert MTB/RIF}

Were the index test results interpreted without knowl- Yes edge of the results of the reference standard?

If a threshold was used, was it pre-specified? Yes

Low Low

\section{DOMAIN 3: Reference Standard}

Is the reference standards likely to correctly classify Yes the target condition?

Were the reference standard results for TB detection Yes interpreted without knowledge of the results of the index test?

Were the reference standard results for rifampicin resistance detection interpreted without knowledge of the results of the index test?

Yes

\begin{tabular}{llll}
\hline & Low & Low \\
\hline DOMAIN 4: Flow and Timing & \\
\hline $\begin{array}{l}\text { Was there an appropriate interval between index test } \\
\text { and reference standard? }\end{array}$ & Yes \\
\hline Did all patients receive the same reference standard? & Yes \\
\hline Were all patients included in the analysis? & Yes
\end{tabular}

Low

\section{Kurbatova 2013}

\section{Study characteristics}

Patient sampling Cross-sectional design, consecutive enrolment, prospective data collection 
Kurbatova 2013 (Continued)

Age: 18 years and older

Sex, female: not reported

HIV infection: estimated $<5 \%$

History of TB: not reported

Sample size: 228

Clinical setting: outpatient and inpatient

Laboratory level: central

Country: Russia

World Bank Income Classification: middle income

High TB burden country: yes

High MDR-TB burden country: no

High TB/HIV burden country: yes

TB incidence rate: 97 per 100,000

MDR-TB prevalence: Percent MDR-TB among new TB cases $=20 \%$

(Source: Surveillance in 20 Oblasts 2010) and among retreatment cases

$=46 \%$ (Source: Surveillance in 20 Oblasts 2008)

Prevalence of TB cases in the study: $46.9 \%$

\begin{tabular}{ll}
\hline Index tests & Index: Xpert MTB/RIF \\
\hline Target condition and reference standard(s) & Target condition: pulmonary TB \\
& Reference standard for pulmonary TB: MGIT 960 \\
& Target condition: rifampicin resistance \\
& Reference standard for rifampicin resistance: MGIT 960
\end{tabular}

Flow and timing

\section{Comparative}

Notes

Fresh, unconcentrated sputum was initially homogenized using a vortex with glass beads

\section{Methodological quality}

\begin{tabular}{llll}
\hline Item & Authors' judgement & Risk of bias & $\begin{array}{l}\text { Applicability con- } \\
\text { cerns }\end{array}$ \\
\hline
\end{tabular}

\section{DOMAIN 1: Patient Selection}

Was a consecutive or random sample of patients en- Yes rolled?

\begin{tabular}{ll}
\hline Was a case-control design avoided? & Yes \\
\hline Did the study avoid inappropriate exclusions? & Yes \\
\hline
\end{tabular}


Kurbatova 2013 (Continued)

Low

Low

\title{
DOMAIN 2: Index Test Xpert MTB/RIF
}

Were the index test results interpreted without knowledge Yes

of the results of the reference standard?

\begin{tabular}{lll}
\hline If a threshold was used, was it pre-specified? & Yes & \\
\hline Low & Low
\end{tabular}

\section{DOMAIN 3: Reference Standard}

Is the reference standards likely to correctly classify the Yes target condition?

Were the reference standard results for TB detection inter- Yes preted without knowledge of the results of the index test?

Were the reference standard results for rifampicin resis- $\quad$ Yes tance detection interpreted without knowledge of the results of the index test?

\section{DOMAIN 4: Flow and Timing}

\section{Was there an appropriate interval between index test and Yes} reference standard?

Did all patients receive the same reference standard? Yes

Were all patients included in the analysis? Yes

\section{Low}

\section{Kwak 2013}

\section{Study characteristics}

Patient sampling

Cross-sectional design, consecutive enrolment, retrospective data collection

Patient characteristics and setting

\author{
Presenting signs and symptoms: people presumed to have pul- \\ monary TB \\ Age: adults > 15 years, median 61 years (IQR 47.5 to 73 ) \\ Sex, female: $37 \%$ \\ HIV infection: $0.7 \%$ \\ History of TB: not reported \\ Sample size: 681 \\ Clinical setting: both outpatient and inpatient
}


Kwak 2013 (Continued)

Laboratory level: central

Country: Republic of Korea

World Bank Income Classification: high income

High TB burden country: no

High MDR-TB burden country: no

High TB/HIV burden country: no

Prevalence of TB cases in the study: $22.9 \%$

Index tests

Target condition and reference standard(s)
Index: Xpert MTB/RIF

Target condition: pulmonary TB

Reference standard for pulmonary TB: Ogawa and MGIT 960

Target condition: rifampicin resistance

Reference standard for rifampicin resistance: LJ by method of absolute concentration

Flow and timing

Comparative

Notes

\section{Methodological quality}

\begin{tabular}{llll}
\hline Item & $\begin{array}{l}\text { Authors' judge- } \\
\text { ment }\end{array}$ & Risk of bias & $\begin{array}{l}\text { Applicability con- } \\
\text { cerns }\end{array}$
\end{tabular}

\section{DOMAIN 1: Patient Selection}

\begin{tabular}{lcc}
\hline Was a consecutive or random sample of patients enrolled? & Yes \\
\hline Was a case-control design avoided? & Yes & \\
\hline Did the study avoid inappropriate exclusions? & Yes & Low \\
\hline
\end{tabular}

\section{DOMAIN 2: Index Test Xpert MTB/RIF}

Were the index test results interpreted without knowledge of the Yes results of the reference standard?

\begin{tabular}{lcc}
\hline If a threshold was used, was it pre-specified? & Yes & Low \\
\hline
\end{tabular}

\section{DOMAIN 3: Reference Standard}

Is the reference standards likely to correctly classify the target Yes condition? 
Kwak 2013 (Continued)

Were the reference standard results for TB detection interpreted Yes without knowledge of the results of the index test?

Were the reference standard results for rifampicin resistance detection interpreted without knowledge of the results of the index test?

Yes

\begin{tabular}{lcc}
\hline LomAIN 4: Flow and Timing & Low \\
\hline Was there an appropriate interval between index test and refer- & Yes \\
ence standard? & Yes & Yes \\
\hline Did all patients receive the same reference standard? & & Low \\
\hline Were all patients included in the analysis? &
\end{tabular}

\section{LaCourse 2016}

\section{Study characteristics}

Patient sampling

\section{Patient characteristics and setting}

Cross-sectional design, consecutive enrolment, prospective data collection

Presenting signs and symptoms: none reported. HIV-infected women accessing prevention of mother-to-child transmission services as part of antenatal care were eligible

Age: 16 years and older, median 25 years (IQR 22 to 30 )

Sex, female: $100 \%$

HIV infection: $100 \%$

History of TB: $9 \%$

Sample size: 288

Clinical setting: outpatient

Laboratory level: central

Country: Kenya

World Bank Income Classification: middle income

High TB burden country: yes

High MDR-TB burden country: yes

High TB/HIV burden country: yes

Prevalence of TB cases in the study: $2.4 \%$ 
LaCourse 2016 (Continued)
Target condition and reference standard(s)
Target condition: pulmonary TB
Reference standard for pulmonary TB: MGIT 960

Flow and timing

Comparative

Notes

Methodological quality

\begin{tabular}{lll}
\hline Item & $\begin{array}{l}\text { Authors' judge- } \\
\text { ment }\end{array}$ & $\begin{array}{c}\text { Risk of bias } \\
\text { concerns }\end{array}$ \\
\hline DOMAIN 1: Patient Selection & Yes \\
\hline Was a consecutive or random sample of patients enrolled? & Yes \\
\hline Was a case-control design avoided? & Yes & Low \\
\hline Did the study avoid inappropriate exclusions? & & Low \\
\hline
\end{tabular}

DOMAIN 2: Index Test Xpert MTB/RIF

Were the index test results interpreted without knowledge of the re- Yes sults of the reference standard?

If a threshold was used, was it pre-specified? Yes

\begin{tabular}{|c|c|c|}
\hline & Low & Low \\
\hline \multicolumn{3}{|l|}{ DOMAIN 3: Reference Standard } \\
\hline $\begin{array}{l}\text { Is the reference standards likely to correctly classify the target condi- } \\
\text { tion? }\end{array}$ & Yes & \\
\hline $\begin{array}{l}\text { Were the reference standard results for TB detection interpreted } \\
\text { without knowledge of the results of the index test? }\end{array}$ & Yes & \\
\hline \multirow[t]{2}{*}{$\begin{array}{l}\text { Were the reference standard results for rifampicin resistance detec- } \\
\text { tion interpreted without knowledge of the results of the index test? }\end{array}$} & Yes & \\
\hline & Low & Low \\
\hline
\end{tabular}

\section{DOMAIN 4: Flow and Timing}

Was there an appropriate interval between index test and reference Yes standard?

Did all patients receive the same reference standard? Yes

Were all patients included in the analysis? Yes 


\section{Lawn 2011}

\section{Study characteristics}

\begin{tabular}{|c|c|}
\hline Patient sampling & Cross-sectional design, consecutive enrolment, prospective data collection \\
\hline \multirow[t]{16}{*}{ Patient characteristics and setting } & $\begin{array}{l}\text { Presenting signs and symptoms: HIV-infected people with advanced immunode- } \\
\text { ficiency; most had } 1 \text { or more of the following TB symptoms: current cough, fever, } \\
\text { night sweats, or weight loss }\end{array}$ \\
\hline & Age: median 34 years (IQR 28 to 41 ) \\
\hline & Sex, female: $65.4 \%$ \\
\hline & HIV infection: $100 \%$ \\
\hline & History of TB: $26.5 \%$ \\
\hline & Sample size: 394 \\
\hline & Clinical setting: HIV anti-retroviral clinic; all participants were screened for TB \\
\hline & Laboratory level: central \\
\hline & Country: South Africa, Cape Town \\
\hline & World Bank Income Classification: middle income \\
\hline & High TB burden country: yes \\
\hline & High MDR-TB burden country: yes \\
\hline & High TB/HIV burden country: yes \\
\hline & TB incidence rate: 993 per 100,000 \\
\hline & $\begin{array}{l}\text { MDR-TB prevalence: } \% \text { MDR-TB among new TB cases }=0.9 \% \text { (Source: survey in West- } \\
\text { ern Cape Province, 2002) and among retreatment cases }=4.0 \% \text { (Source: survey in } \\
\text { Western Cape Province, 2002) }\end{array}$ \\
\hline & Prevalence of TB cases in the study: $18.3 \%$ \\
\hline
\end{tabular}

\begin{tabular}{ll}
\hline Index tests & Index: Xpert MTB/RIF \\
\hline
\end{tabular}

Target condition and reference standard(s) Target condition: pulmonary TB

Reference standard for pulmonary TB: MGIT 960

Target condition: rifampicin resistance

Reference standard for rifampicin resistance: MGIT 960

Flow and timing

Comparative

This study evaluated the use of Xpert to screen HIV-infected people with advanced immunodeficiency enrolling in antiretroviral therapy services regardless of symptoms, although most participants in the study had TB symptoms. Of 3 participants with apparent false-positive Xpert MTB/RIF results, on follow-up 2 had overt pulmonary and systemic symptoms suggestive of TB and improved on anti-TB treatment. The 3rd participant was lost to follow-up.

Median CD4 cell count, 171 cells/ml; IQR 102 to 236 
Lawn 2011 (Continued)

Methodological quality

\begin{tabular}{llll}
\hline Item & Authors' judgement & Risk of bias & Applicability concerns \\
\hline
\end{tabular}

DOMAIN 1: Patient Selection

Was a consecutive or random sample of pa- Yes tients enrolled?

Was a case-control design avoided? Yes

Did the study avoid inappropriate exclusions? Yes

Low Low

\section{DOMAIN 2: Index Test Xpert MTB/RIF}

Were the index test results interpreted with- Yes out knowledge of the results of the reference standard?

If a threshold was used, was it pre-specified? Yes

\begin{tabular}{lll}
\hline Low & Low \\
\hline DOMAIN 3: Reference Standard &
\end{tabular}

Is the reference standards likely to correctly Yes

classify the target condition?

Were the reference standard results for TB de- Yes

tection interpreted without knowledge of the

results of the index test?

Were the reference standard results for ri- Yes

fampicin resistance detection interpreted

without knowledge of the results of the index

test?

\begin{tabular}{lll}
\hline Low & Low \\
\hline DOMAIN 4: Flow and Timing & & \\
\hline $\begin{array}{l}\text { Was there an appropriate interval between in- } \\
\text { dex test and reference standard? }\end{array}$ & Yes \\
\hline $\begin{array}{l}\text { Did all patients receive the same reference } \\
\text { standard? }\end{array}$ & Yes \\
\hline Were all patients included in the analysis? & Low \\
\hline
\end{tabular}




\section{Le Palud 2014}

\section{Study characteristics}

Patient sampling

Cross-sectional design, consecutive enrolment, retrospective data collection

Patient characteristics and setting

Presenting signs and symptoms: presumptive pulmonary TB based on clinical features (e.g. cough, haemoptysis, fever, asthenia, loss of weight, and night sweats) or radiological features (e.g. nodule, pneumonia, cavitation, and pleurisy), smear-negative

Age: median 54 years (IQR 34 to 74 )

Sex, female: $37 \%$

HIV infection: $4 \%$

History of TB: not reported

Sample size: 162

Clinical setting: not reported

Laboratory level: central

Country: France

World Bank Income Classification: high income

High TB burden country: no

High MDR-TB burden country: no

High TB/HIV burden country: no

Prevalence of TB cases in the study: $12.3 \%$

Target condition and reference standard(s)

Target condition: pulmonary TB

Reference standard for pulmonary TB: Colestos slant and MGIT 960

Target condition: rifampicin resistance

Reference standard for rifampicin resistance: MGIT 960

Flow and timing

Comparative

Notes

\section{Methodological quality}

\section{Item}

Authors' judgement Risk of bias

Applicability con-

cerns

\section{DOMAIN 1: Patient Selection}

Was a consecutive or random sample of patients enrolled?

Yes 
Le Palud 2014 (Continued)

Was a case-control design avoided?

Yes

Did the study avoid inappropriate exclusions?

No

\begin{tabular}{llll}
\hline DOMAIN 2: Index Test Xpert MTB/RIF & High & Unclear \\
\hline $\begin{array}{l}\text { Were the index test results interpreted without knowledge of } \\
\text { the results of the reference standard? }\end{array}$ & Yes & \\
\hline If a threshold was used, was it pre-specified? & & Low \\
\hline
\end{tabular}

\section{DOMAIN 3: Reference Standard}

Is the reference standards likely to correctly classify the target Yes condition?

Were the reference standard results for TB detection interpreted without knowledge of the results of the index test?

Were the reference standard results for rifampicin resistance detection interpreted without knowledge of the results of the index test?

\section{Yes}

\section{DOMAIN 4: Flow and Timing}

Was there an appropriate interval between index test and ref- Yes erence standard?

Did all patients receive the same reference standard? Yes

Were all patients included in the analysis? Yes

Low

\section{Lee 2013}

\section{Study characteristics}

Patient sampling

Cross-sectional design, consecutive enrolment, retrospective data collection

Patient characteristics and setting

Presenting signs and symptoms: presumed pulmonary TB, smear-negative

Age: median: 54 years, range 18 to 90 years

Sex, female: $41 \%$

HIV infection: $1 \%$ 
Lee 2013 (Continued)

History of TB: $21 \%$

Sample size: 132

Clinical setting: not reported

Laboratory level: central

Country: Republic of Korea

World Bank Income Classification: high income

High TB burden country: no

High MDR-TB burden country: no

High TB/HIV burden country: no

Prevalence of TB cases in the study: $28.8 \%$

\begin{tabular}{ll}
\hline Index tests & Index: Xpert MTB/RIF \\
\hline Target condition and reference standard(s) & Target condition: pulmonary TB \\
& $\begin{array}{l}\text { Reference standard for pulmonary TB: Ogawa medium and } \\
\text { MGIT } 960\end{array}$ \\
& Target condition: rifampicin resistance \\
& $\begin{array}{l}\text { Reference standard for rifampicin resistance: Ogawa medium, } \\
\text { proportion method }\end{array}$
\end{tabular}

Flow and timing

Comparative

Notes

\section{Methodological quality}

\begin{tabular}{lll}
\hline Item & $\begin{array}{l}\text { Authors' judge- } \\
\text { ment }\end{array}$ & Risk of bias $\begin{array}{l}\text { Applicability con- } \\
\text { cerns }\end{array}$ \\
\hline
\end{tabular}

\section{DOMAIN 1: Patient Selection}

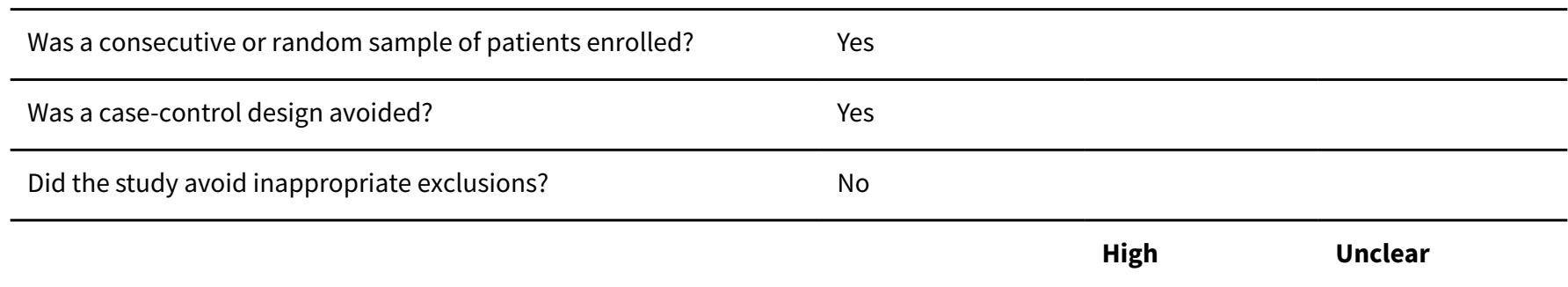

\section{DOMAIN 2: Index Test Xpert MTB/RIF}

Were the index test results interpreted without knowledge of the re- Yes sults of the reference standard?

\begin{tabular}{ll}
\hline If a threshold was used, was it pre-specified? & Yes \\
\hline Low \\
\hline \hline
\end{tabular}

Xpert MTB/RIF and Xpert MTB/RIF Ultra for pulmonary tuberculosis and rifampicin resistance in adults (Review) 
Lee 2013 (Continued)

DOMAIN 3: Reference Standard

Is the reference standards likely to correctly classify the target con- Yes dition?

Were the reference standard results for TB detection interpreted Unclear without knowledge of the results of the index test?

Were the reference standard results for rifampicin resistance detection interpreted without knowledge of the results of the index test?

\begin{tabular}{lll}
\hline & Unclear & Low \\
\hline DOMAIN 4: Flow and Timing & & \\
\hline Was there an appropriate interval between index test and reference & Yes \\
standard? & Yes \\
\hline Did all patients receive the same reference standard? & Yes \\
\hline Were all patients included in the analysis?
\end{tabular}

Low

\section{Lippincott 2014}

\section{Study characteristics}

Patient sampling

Cross-sectional design, consecutive enrolment, retrospective data collection

Patient characteristics and setting

Presenting signs and symptoms: presumptive pulmonary TB

Age: median: 51 years (IQR 39 to 63 )

Sex, female: $36 \%$

HIV infection: $24 \%$

History of TB: not reported

Sample size: 499

Clinical setting: inpatient

Laboratory level: central

Country: USA

World Bank Income Classification: high income

High TB burden country: no

High MDR-TB burden country: no

High TB/HIV burden country: no 
Lippincott 2014 (Continued)

Prevalence of TB cases in the study: $3.0 \%$

Index tests

Target condition and reference standard(s)
Index: Xpert MTB/RIF

Target condition: pulmonary TB

Reference standard for pulmonary TB: LJ and MGIT 960

Target condition: rifampicin resistance

Reference standard for rifampicin resistance: MGIT 960

Flow and timing

Comparative

Notes

Methodological quality

\begin{tabular}{lll}
\hline Item & $\begin{array}{l}\text { Authors' judge- } \\
\text { ment }\end{array}$ & $\begin{array}{c}\text { Risk of bias } \\
\text { concerns }\end{array}$ \\
\hline DOMAIN 1: Patient Selection & Yes \\
\hline Was a consecutive or random sample of patients enrolled? & Yes \\
\hline Was a case-control design avoided? & Yes & Low \\
\hline Did the study avoid inappropriate exclusions? & & High
\end{tabular}

DOMAIN 2: Index Test Xpert MTB/RIF

Were the index test results interpreted without knowledge of the re- Yes sults of the reference standard?

If a threshold was used, was it pre-specified? Yes

\begin{tabular}{llll}
\hline DOMAIN 3: Reference Standard & Low & \\
\hline Is the reference standards likely to correctly classify the target condi- & Yes \\
tion? & & Yes \\
\hline $\begin{array}{l}\text { Were the reference standard results for TB detection interpreted with- } \\
\text { out knowledge of the results of the index test? }\end{array}$ & Yes \\
\hline $\begin{array}{l}\text { Were the reference standard results for rifampicin resistance detec- } \\
\text { tion interpreted without knowledge of the results of the index test? }\end{array}$ & Low \\
\hline
\end{tabular}

DOMAIN 4: Flow and Timing 
Lippincott 2014 (Continued)

Was there an appropriate interval between index test and reference Yes standard?

\begin{tabular}{ll}
\hline Did all patients receive the same reference standard? & Yes \\
\hline
\end{tabular}

Were all patients included in the analysis?

Yes

Low

\section{Liu 2017}

\section{Study characteristics}

\section{Patient sampling}

\section{Patient characteristics and setting}

Cross-sectional design, consecutive enrolment, prospective data collection

Presenting signs and symptoms: people presumed to have pulmonary TB, who had cough, expectoration or haemoptysis for more than 2 weeks were enrolled

Age: 15 years and older

Sex, female: not reported

HIV infection: not reported

History of TB: not reported

Sample size: 3096

Clinical setting: not reported

Laboratory level: intermediate

Country: China

World Bank Income Classification: middle income

High TB burden country: yes

High MDR-TB burden country: yes

High TB/HIV burden country: yes

Prevalence of TB cases in the study: 14.1\%

Index tests

Index: Xpert MTB/RIF

Target condition and reference standard(s)

Target condition: pulmonary TB

Reference standard for pulmonary TB: LJ

Target condition: rifampicin resistance

Reference standard for rifampicin resistance: LJ

\section{Flow and timing}


Liu 2017 (Continued)

Notes

\section{Methodological quality}

\begin{tabular}{llll}
\hline Item & $\begin{array}{l}\text { Authors' judge- } \\
\text { ment }\end{array}$ & Risk of bias & $\begin{array}{l}\text { Applicability con- } \\
\text { cerns }\end{array}$
\end{tabular}

\section{DOMAIN 1: Patient Selection}

\begin{tabular}{lcc}
\hline Was a consecutive or random sample of patients enrolled? & Yes \\
\hline Was a case-control design avoided? & Yes & \\
\hline Did the study avoid inappropriate exclusions? & Yes & Low \\
\hline
\end{tabular}

\section{DOMAIN 2: Index Test Xpert MTB/RIF}

Were the index test results interpreted without knowledge of the Yes results of the reference standard?

If a threshold was used, was it pre-specified? Yes

\begin{tabular}{lcc}
\hline & Low & Low \\
\hline DOMAIN 3: Reference Standard &
\end{tabular}

\section{DOMAIN 3: Reference Standard}

Is the reference standards likely to correctly classify the target Yes condition?

Were the reference standard results for TB detection interpreted Yes without knowledge of the results of the index test?

Were the reference standard results for rifampicin resistance detection interpreted without knowledge of the results of the index test?

\section{DOMAIN 4: Flow and Timing}

Was there an appropriate interval between index test and refer- Yes ence standard?

Did all patients receive the same reference standard? Yes

Were all patients included in the analysis? Yes

Low

\section{Study characteristics}


Lorent 2015 (Continued)

Patient sampling

Patient characteristics and setting

Cross-sectional design, consecutive enrolment, prospective data collection

Presenting signs and symptoms: presumptive drug-resistant TB, including previously treated people (failure, relapse, return after default); symptomatic close contacts of known MDR-TB cases; new TB patients with delayed smear conversion at month 2 or 3 of first-line treatment; and all HIV-infected people, regardless of smear results

Age: median: 43 years (IQR 34 to 52)

Sex, female: $47 \%$

HIV infection: $65 \%$

History of TB: $46 \%$

Sample size: 274

Clinical setting: outpatient

Laboratory level: central

Country: Cambodia

World Bank Income Classification: middle income

High TB burden country: yes

High MDR-TB burden country: no

High TB/HIV burden country: no

\begin{tabular}{ll}
\hline Index tests & Index: Xpert MTB/RIF \\
\hline Target condition and reference standard(s) & Target condition: rifampicin resistance \\
& $\begin{array}{l}\text { Reference standard for rifampicin resistance: LJ proportion } \\
\text { method }\end{array}$
\end{tabular}

Flow and timing

Comparative

Notes

\section{Methodological quality}

Item

Authors' judgement Risk of bias

\section{Applicability con-} cerns

DOMAIN 1: Patient Selection

Was a consecutive or random sample of patients enrolled?

Was a case-control design avoided?

Did the study avoid inappropriate exclusions?
Yes

Yes

Yes 
Lorent 2015 (Continued)

\section{DOMAIN 2: Index Test Xpert MTB/RIF}

Were the index test results interpreted without knowledge of Yes the results of the reference standard?

If a threshold was used, was it pre-specified? Yes

Low

Low

\section{DOMAIN 3: Reference Standard}

Is the reference standards likely to correctly classify the target Yes condition?

Were the reference standard results for TB detection interpret- No ed without knowledge of the results of the index test?

Were the reference standard results for rifampicin resistance No detection interpreted without knowledge of the results of the index test?

\begin{tabular}{lll}
\hline & High & Low \\
\hline DOMAIN 4: Flow and Timing & & \\
\hline $\begin{array}{l}\text { Was there an appropriate interval between index test and refer- } \\
\text { ence standard? }\end{array}$ & Yes \\
\hline Did all patients receive the same reference standard? & Yes \\
\hline Were all patients included in the analysis? & & Low \\
\hline
\end{tabular}

\section{Luetkemeyer 2016}

\section{Study characteristics}

Patient sampling

Cross-sectional design, unknown manner of enrolment, prospective data collection

Patient characteristics and setting

Presenting signs and symptoms: cough, fever, night sweats, or weight loss

Age: 18 years and older, median 46 years (IQR 35 to 54 )

Sex, female: $38 \%$

HIV infection: $45 \%$

History of TB: $13 \%$

Sample size: 992

Clinical setting: inpatient and outpatient

Laboratory level: central 
Country: Brazil, South Africa and USA

World Bank Income Classification: high and middle income

High TB burden country: yes (South Africa), no (USA)

High MDR-TB burden country: yes (South Africa), no (USA)

High TB/HIV burden country: yes (South Africa), no (USA)

Prevalence of TB cases in the study: 22.4

\begin{tabular}{ll}
\hline Index tests & Index: Xpert MTB/RIF \\
\hline Target condition and reference standard(s) & Target condition: pulmonary TB \\
& Reference standard for pulmonary TB: solid media and MGIT 960 \\
& Target condition: rifampicin resistance \\
& Reference standard for rifampicin resistance: Middlebrook agar
\end{tabular}

Flow and timing

Comparative

Notes

\section{Methodological quality}

\begin{tabular}{llll}
\hline Item Authors' judgement & Risk of bias & $\begin{array}{l}\text { Applicability con- } \\
\text { cerns }\end{array}$
\end{tabular}

\section{DOMAIN 1: Patient Selection}

\begin{tabular}{llll}
\hline Was a consecutive or random sample of patients enrolled? & Unclear \\
\hline Was a case-control design avoided? & Yes & \\
\hline Did the study avoid inappropriate exclusions? & Yes & Unclear & Low \\
\hline
\end{tabular}

\section{DOMAIN 2: Index Test Xpert MTB/RIF}

Were the index test results interpreted without knowledge of Yes the results of the reference standard?

\begin{tabular}{lcc}
\hline If a threshold was used, was it pre-specified? & Yes & \\
\hline Low & Low \\
\hline
\end{tabular}

\section{DOMAIN 3: Reference Standard}

Is the reference standards likely to correctly classify the target Yes condition?

Were the reference standard results for TB detection interYes preted without knowledge of the results of the index test? 
Luetkemeyer 2016 (Continued)

Were the reference standard results for rifampicin resistance detection interpreted without knowledge of the results of the index test?

\begin{tabular}{lcc}
\hline & Low & Low \\
\hline DOMAIN 4: Flow and Timing & &
\end{tabular}

Was there an appropriate interval between index test and ref- Yes erence standard?

\begin{tabular}{ll}
\hline Did all patients receive the same reference standard? & Yes \\
\hline Were all patients included in the analysis? & Yes \\
\hline
\end{tabular}

\section{Makamure 2017}

\section{Study characteristics}

Patient sampling

Cross-sectional design, enrolment by convenience, prospective data collection

Patient characteristics and setting

Presenting signs and symptoms: MDR-TB high-risk patients (TB symptoms with at least 1 of the following: previously confirmed MDR-TB, failure to convert after at least 2 months therapy, treatment failure, return after default, relapse after completion of treatment or contacts of known MDR-TB cases)

Age: 15 years and older, median: 38 years (IQR 30 to 47 )

Sex, female: $42 \%$

HIV infection: $63 \%$

History of TB: $78 \%$

Sample size: 210

Clinical setting: not reported

Laboratory level: central

Country: Zimbabwe

World Bank Income Classification: low income

High TB burden country: yes

High MDR-TB burden country: yes

High TB/HIV burden country: yes

Index tests Index: Xpert MTB/RIF

Target condition and reference standard(s)

Target condition: rifampicin resistance

Reference standard for rifampicin resistance: LJ 
Makamure 2017 (Continued)

Flow and timing

\section{Comparative}

Notes

\section{Methodological quality}

\begin{tabular}{llll}
\hline Item & $\begin{array}{l}\text { Authors' judge- } \\
\text { ment }\end{array}$ & Risk of bias & $\begin{array}{l}\text { Applicability con- } \\
\text { cerns }\end{array}$
\end{tabular}

\section{DOMAIN 1: Patient Selection}

\begin{tabular}{ll}
\hline Was a consecutive or random sample of patients enrolled? & No \\
\hline Was a case-control design avoided? & Yes \\
\hline Did the study avoid inappropriate exclusions? & Yes \\
\hline
\end{tabular}

\begin{tabular}{lccc}
\hline DOMAIN 2: Index Test Xpert MTB/RIF & High & Unclear \\
\hline $\begin{array}{l}\text { Were the index test results interpreted without knowledge of the } \\
\text { results of the reference standard? }\end{array}$ & Yes & \\
\hline If a threshold was used, was it pre-specified? & Low & Low \\
\hline
\end{tabular}

\section{DOMAIN 3: Reference Standard}

Is the reference standards likely to correctly classify the target Yes condition?

Were the reference standard results for TB detection interpreted without knowledge of the results of the index test?

Were the reference standard results for rifampicin resistance detection interpreted without knowledge of the results of the index test?

\begin{tabular}{llll}
\hline DOMAIN 4: Flow and Timing & Low & Low \\
\hline $\begin{array}{l}\text { Was there an appropriate interval between index test and refer- } \\
\text { ence standard? }\end{array}$ & Yes & Yes \\
\hline Did all patients receive the same reference standard? & Yes & Low
\end{tabular}


Mbelele 2017

\section{Study characteristics}

\section{Patient sampling}

\section{Patient characteristics and setting}

Cross-sectional design, unknown manner of enrolment, prospective data collection

Presenting signs and symptoms: presumed pulmonary TB

Age: 18 years and older, mean 43 years (SD 15)

Sex, female: $66 \%$

HIV infection: $15 \%$

History of TB: $14 \%$

Sample size: 262

Clinical setting: not reported

Laboratory level: central

Country: Tanzania

World Bank Income Classification: low income

High TB burden country: yes

High MDR-TB burden country: no

High TB/HIV burden country: yes

Prevalence of TB cases in the study: $32.4 \%$

Index: Xpert MTB/RIF

Target condition: pulmonary TB

Reference standard for pulmonary TB: LJ

Flow and timing

Comparative

$$
\text { Notes }
$$

\section{Methodological quality}

\begin{tabular}{lll}
\hline Item & $\begin{array}{l}\text { Authors' judge- } \\
\text { ment }\end{array}$ & $\begin{array}{l}\text { Risk of bias } \\
\text { Applicability } \\
\text { concerns }\end{array}$ \\
\hline
\end{tabular}

\section{DOMAIN 1: Patient Selection}

\begin{tabular}{lccl}
\hline Was a consecutive or random sample of patients enrolled? & Unclear \\
\hline Was a case-control design avoided? & Yes & Yes & Unclear \\
\hline Did the study avoid inappropriate exclusions? & & Unclear \\
\hline
\end{tabular}

Xpert MTB/RIF and Xpert MTB/RIF Ultra for pulmonary tuberculosis and rifampicin resistance in adults (Review) 
Mbelele 2017 (Continued)

\section{DOMAIN 2: Index Test Xpert MTB/RIF}

Were the index test results interpreted without knowledge of the results Yes of the reference standard?

If a threshold was used, was it pre-specified? Yes

\begin{tabular}{lll}
\hline DOMAIN 3: Reference Standard & Low & \\
\hline Is the reference standards likely to correctly classify the target condition? Yes & Unclear & Unclear \\
\hline $\begin{array}{l}\text { Were the reference standard results for TB detection interpreted without } \\
\text { knowledge of the results of the index test? }\end{array}$ & Yes \\
\hline $\begin{array}{l}\text { Were the reference standard results for rifampicin resistance detection } \\
\text { interpreted without knowledge of the results of the index test? }\end{array}$ & Low \\
\hline DomAIN 4: Flow and Timing & Yes \\
\hline Was there an appropriate interval between index test and reference stan- \\
\hline dard?
\end{tabular}

Meawed 2016

\section{Study characteristics}

Patient sampling

Patient characteristics and setting
Cross-sectional design, unknown manner of enrolment, prospective data collection

Presenting signs and symptoms: recurrence of general or local chest symptoms, suspected retreatment TB

Age: mean 33 years (SD 19), age range 21 - 67 years

Sex, female: $33 \%$

HIV infection: not reported

History of TB: $100 \%$

Sample size: 58

Clinical setting: outpatient

Laboratory level: central

Country: Egypt 
High TB burden country: no

High MDR-TB burden country: no

High TB/HIV burden country: no

Prevalence of TB cases in the study: $93.1 \%$

\begin{tabular}{ll}
\hline Index tests & Index: Xpert MTB/RIF \\
\hline Target condition and reference standard(s) & Target condition: pulmonary TB \\
& Reference standard for pulmonary TB: LJ \\
& Target condition: rifampicin resistance \\
& Reference standard for rifampicin resistance: MGIT 960
\end{tabular}

Flow and timing

Comparative

Notes

\section{Methodological quality}

\begin{tabular}{|c|c|c|c|}
\hline Item & $\begin{array}{l}\text { Authors' judge- } \\
\text { ment }\end{array}$ & Risk of bias & $\begin{array}{l}\text { Applicability con- } \\
\text { cerns }\end{array}$ \\
\hline
\end{tabular}

\section{DOMAIN 1: Patient Selection}

\begin{tabular}{lccl}
\hline Was a consecutive or random sample of patients enrolled? & Unclear \\
\hline Was a case-control design avoided? & Yes & No & High \\
\hline Did the study avoid inappropriate exclusions? & Low \\
\hline
\end{tabular}

\section{DOMAIN 2: Index Test Xpert MTB/RIF}

Were the index test results interpreted without knowledge of the Yes results of the reference standard?

\begin{tabular}{lll}
\hline If a threshold was used, was it pre-specified? & Yes & Low \\
\hline DOMAIN 3: Reference Standard & Yes \\
\hline $\begin{array}{l}\text { Is the reference standards likely to correctly classify the target } \\
\text { condition? }\end{array}$ & Unclear \\
\hline $\begin{array}{l}\text { Were the reference standard results for TB detection interpreted } \\
\text { without knowledge of the results of the index test? }\end{array}$
\end{tabular}


Meawed 2016 (Continued)

Were the reference standard results for rifampicin resistance detection interpreted without knowledge of the results of the index test?

Unclear

Low

\section{DOMAIN 4: Flow and Timing}

Was there an appropriate interval between index test and refer- Yes ence standard?

Did all patients receive the same reference standard? Yes

Were all patients included in the analysis? Yes

Low

\section{Metcalfe 2015}

\section{Study characteristics}

Patient sampling

Cross-sectional design, consecutive enrolment, prospective data collection

Patient characteristics and setting

Presenting signs and symptoms: recurrent TB (TB following cure or completion of treatment of a previous TB episode), or prevalent retreatment TB (treatment failure, i.e. sputum smear-positivity at month 5 or later)

Age: 15 years and older

Sex, female: not reported

HIV infection: $75 \%$

History of TB: $100 \%$

Sample size: 149

Clinical setting: outpatient

Laboratory level: central

Country: Zimbabwe

World Bank Income Classification: low income

High TB burden country: yes

High MDR-TB burden country: yes

High TB/HIV burden country: yes

Prevalence of TB cases in the study: 59.7

\begin{tabular}{ll}
\hline Index tests $\quad$ Index: Xpert MTB/RIF &
\end{tabular}

\footnotetext{
Target condition and reference standard(s)
}

Target condition: pulmonary TB 
Metcalfe 2015 (Continued)

Flow and timing

Comparative

Notes

\section{Methodological quality}

\begin{tabular}{lll}
\hline Item & $\begin{array}{l}\text { Authors' judge- } \\
\text { ment }\end{array}$ & Risk of bias $\begin{array}{l}\text { Applicability con- } \\
\text { cerns }\end{array}$
\end{tabular}

\section{DOMAIN 1: Patient Selection}

\begin{tabular}{lc}
\hline Was a consecutive or random sample of patients enrolled? & Yes \\
\hline Was a case-control design avoided? & Yes \\
\hline
\end{tabular}

Did the study avoid inappropriate exclusions?

No

High Low

\section{DOMAIN 2: Index Test Xpert MTB/RIF}

Were the index test results interpreted without knowledge of the re- Yes sults of the reference standard?

\begin{tabular}{lcc}
\hline If a threshold was used, was it pre-specified? & Yes \\
\hline Low & Low \\
\hline
\end{tabular}

\section{DOMAIN 3: Reference Standard}

Is the reference standards likely to correctly classify the target con- Yes dition?

Were the reference standard results for TB detection interpreted Yes without knowledge of the results of the index test?

Were the reference standard results for rifampicin resistance detec- Yes tion interpreted without knowledge of the results of the index test?

Low Low

\section{DOMAIN 4: Flow and Timing}

Was there an appropriate interval between index test and reference Yes standard?

\begin{tabular}{lc}
\hline Did all patients receive the same reference standard? & Yes \\
\hline Were all patients included in the analysis? & Yes
\end{tabular}


Metcalfe 2016

\section{Study characteristics}

\section{Patient sampling}

\section{Patient characteristics and setting}

Cross-sectional design, consecutive enrolment, prospective data collection

Presenting signs and symptoms: cough (any duration), fever, night sweats, or weight loss, with a history of prior TB

Age: 15 years and older

Sex, female: not reported

HIV infection: $68 \%$

History of TB: $100 \%$

Sample size: 352

Clinical setting: outpatient

Laboratory level: central

Country: Zimbabwe

World Bank Income Classification: low income

High TB burden country: yes

High MDR-TB burden country: yes

High TB/HIV burden country: yes

\begin{tabular}{ll}
\hline Index tests & Index: Xpert MTB/RIF \\
\hline Target condition and reference standard(s) & Target condition: rifampicin resistance \\
& Reference standard for rifampicin resistance: LJ and \\
& MODS
\end{tabular}

Flow and timing

Comparative

Notes

\section{Methodological quality}

\begin{tabular}{lll}
\hline Item & $\begin{array}{l}\text { Authors' judge- } \\
\text { ment }\end{array}$ & Risk of bias \\
\hline DOMAIN 1: Patient Selection & $\begin{array}{c}\text { Applicability } \\
\text { concerns }\end{array}$ \\
\hline Was a consecutive or random sample of patients enrolled? & Yes \\
\hline Was a case-control design avoided? & Yes \\
\hline Did the study avoid inappropriate exclusions? & Yes \\
\hline
\end{tabular}


Metcalfe 2016 (Continued)

Low

Low

\section{DOMAIN 2: Index Test Xpert MTB/RIF}

Were the index test results interpreted without knowledge of the results of Yes the reference standard?

\begin{tabular}{lcl}
\hline If a threshold was used, was it pre-specified? & Yes & Low Low \\
\hline
\end{tabular}

\section{DOMAIN 3: Reference Standard}

Is the reference standards likely to correctly classify the target condition? Yes

Were the reference standard results for TB detection interpreted without knowledge of the results of the index test?

Were the reference standard results for rifampicin resistance detection in- Yes terpreted without knowledge of the results of the index test?

Low Low

\section{DOMAIN 4: Flow and Timing}

Was there an appropriate interval between index test and reference stan- Yes dard?

\begin{tabular}{lc}
\hline Did all patients receive the same reference standard? & Yes \\
\hline Were all patients included in the analysis? & Yes \\
\hline
\end{tabular}

\section{Meyer 2017}

\section{Study characteristics}

Patient sampling

Cross-sectional design, consecutive enrolment, prospective data collection

Patient characteristics and setting

Presenting signs and symptoms: presumptive TB with cough $\geq 2$ weeks but $<6$ months, smear-negative

Age: 18 years and older, median 34 years (IQR 28 to 44 )

Sex, female: $49 \%$

HIV infection: $66 \%$

History of TB: $12 \%$

Sample size: 1782

Clinical setting: inpatient

Laboratory level: central 
Meyer 2017 (Continued)

Country: Uganda

World Bank Income Classification: low income

High TB burden country: no

High MDR-TB burden country: no

High TB/HIV burden country: yes

Prevalence of TB cases in the study: $22 \%$

\begin{tabular}{ll}
\hline Index tests & Index: Xpert MTB/RIF \\
\hline Target condition and reference standard(s) & Target condition: pulmonary TB \\
& Reference standard for pulmonary TB: LJ \\
\hline
\end{tabular}

Flow and timing

Comparative

Notes

\section{Methodological quality}

\begin{tabular}{lll}
\hline Item & $\begin{array}{l}\text { Authors' judge- } \\
\text { ment }\end{array}$ & $\begin{array}{l}\text { Risk of bias } \\
\text { concerns }\end{array}$
\end{tabular}

\section{DOMAIN 1: Patient Selection}

\begin{tabular}{lcc}
\hline Was a consecutive or random sample of patients enrolled? & Yes & \\
\hline Was a case-control design avoided? & Yes & No \\
\hline Did the study avoid inappropriate exclusions? & High & High \\
\hline
\end{tabular}

\section{DOMAIN 2: Index Test Xpert MTB/RIF}

Were the index test results interpreted without knowledge of the results Yes of the reference standard?

If a threshold was used, was it pre-specified? Yes

\begin{tabular}{lll} 
& Low & Low \\
\hline DOMAIN 3: Reference Standard &
\end{tabular}

Is the reference standards likely to correctly classify the target condi- Yes tion?

Were the reference standard results for TB detection interpreted with- Yes out knowledge of the results of the index test?

Were the reference standard results for rifampicin resistance detection Yes interpreted without knowledge of the results of the index test? 
Meyer 2017 (Continued)

Low

Low

\section{DOMAIN 4: Flow and Timing}

Was there an appropriate interval between index test and reference

Yes

standard?

\begin{tabular}{ll}
\hline Did all patients receive the same reference standard? Yes \\
\hline
\end{tabular}

Were all patients included in the analysis? Yes

Low

\section{Mok 2016}

\section{Study characteristics}

\section{Patient sampling}

Cross-sectional design, consecutive enrolment, retrospective data collection

\section{Patient characteristics and setting}

Presenting signs and symptoms: presumptive pulmonary TB, sputum scarce or sputum smear-negative

Age: 21 years and older, median 59 years (IQR 43 to 66)

Sex, female: $29 \%$

HIV infection: not reported

History of TB: not reported

Sample size: 158

Clinical setting: inpatient

Laboratory level: central

Country: Singapore

World Bank Income Classification: high income

High TB burden country: no

High MDR-TB burden country: no

High TB/HIV burden country: no

Prevalence of TB cases in the study: $28 \%$

Index tests Index: Xpert MTB/RIF

Target condition and reference standard(s)

Target condition: pulmonary TB

Reference standard for pulmonary TB: MGIT 960

Flow and timing

\section{Comparative}


Mok 2016 (Continued)

Notes

\section{Methodological quality}

\begin{tabular}{|c|c|c|c|}
\hline Item & $\begin{array}{l}\text { Authors' judge- } \\
\text { ment }\end{array}$ & Risk of bias & $\begin{array}{l}\text { Applicability } \\
\text { concerns }\end{array}$ \\
\hline
\end{tabular}

\section{DOMAIN 1: Patient Selection}

\begin{tabular}{lc}
\hline Was a consecutive or random sample of patients enrolled? & Yes \\
\hline Was a case-control design avoided? & Yes \\
\hline Did the study avoid inappropriate exclusions? & No
\end{tabular}

\begin{tabular}{lcc}
\hline DOMAIN 2: Index Test Xpert MTB/RIF & High & High \\
\hline $\begin{array}{l}\text { Were the index test results interpreted without knowledge of the results } \\
\text { of the reference standard? }\end{array}$ & Yes & Low \\
\hline If a threshold was used, was it pre-specified? & Unclear
\end{tabular}

\section{DOMAIN 3: Reference Standard}

Is the reference standards likely to correctly classify the target condi- Yes tion?

Were the reference standard results for TB detection interpreted with- Yes out knowledge of the results of the index test?

Were the reference standard results for rifampicin resistance detection Yes interpreted without knowledge of the results of the index test?

\begin{tabular}{lll}
\hline Low & Low & \\
\hline DOMAIN 4: Flow and Timing & Yes & \\
\hline $\begin{array}{l}\text { Was there an appropriate interval between index test and reference } \\
\text { standard? }\end{array}$ & Yes \\
\hline Did all patients receive the same reference standard? & Yes \\
\hline Were all patients included in the analysis? &
\end{tabular}

\section{Low}

\section{Mokaddas 2015}

\section{Study characteristics}




\section{Mokaddas 2015 (Continued)}

Patient sampling

Cross-sectional design, consecutive enrolment, prospective data collection

Patient characteristics and setting

Presenting signs and symptoms: presumptive TB

Age: 14 years and older

Sex, female: not reported

HIV infection: not reported

History of TB: not reported

Sample size: 287

Clinical setting: laboratory-based

Laboratory level: central

Country: Kuwait

World Bank Income Classification: high income

High TB burden country: no

High MDR-TB burden country: no

High TB/HIV burden country: no

Prevalence of TB cases in the study: $21.9 \%$

Index: Xpert MTB/RIF

Target condition: rifampicin resistance

Reference standard for rifampicin resistance: MGIT 960

Flow and timing

Comparative

Notes

\section{Methodological quality}

\begin{tabular}{lll}
\hline Item & $\begin{array}{l}\text { Authors' judge- } \\
\text { ment }\end{array}$ & $\begin{array}{l}\text { Risk of bias } \\
\text { Applicability } \\
\text { concerns }\end{array}$ \\
\hline
\end{tabular}

\section{DOMAIN 1: Patient Selection}

\begin{tabular}{lcc}
\hline Was a consecutive or random sample of patients enrolled? & Yes \\
\hline Was a case-control design avoided? & Yes & Yes \\
\hline Did the study avoid inappropriate exclusions? & Low Unclear
\end{tabular}

\section{DOMAIN 2: Index Test Xpert MTB/RIF}


Mokaddas 2015 (Continued)

Were the index test results interpreted without knowledge of the results of Yes the reference standard?

\begin{tabular}{lcl}
\hline If a threshold was used, was it pre-specified? & Yes & \\
\hline & Low L L L L L \\
\hline
\end{tabular}

\section{DOMAIN 3: Reference Standard}

Is the reference standards likely to correctly classify the target condition? Yes

Were the reference standard results for TB detection interpreted without knowledge of the results of the index test?

Were the reference standard results for rifampicin resistance detection in- Yes terpreted without knowledge of the results of the index test?

Low Low

\section{DOMAIN 4: Flow and Timing}

Was there an appropriate interval between index test and reference stan- Yes dard?

\begin{tabular}{ll}
\hline Did all patients receive the same reference standard? & Yes \\
\hline Were all patients included in the analysis? & Yes \\
\hline
\end{tabular}

\section{Mollel 2017}

\section{Study characteristics}

Patient sampling Cross-sectional design, consecutive enrolment, prospective data collection

Patient characteristics and setting

Presenting signs and symptoms: not reported

Age: 16 years and older, mean 42 years

Sex, female: $55 \%$

HIV infection: $100 \%$

History of TB: not reported

Sample size: 69

Clinical setting: outpatient

Laboratory level: intermediate

Country: Tanzania

World Bank Income Classification: low income

High TB burden country: yes 
Mollel 2017 (Continued)

High MDR-TB burden country: no

High TB/HIV burden country: yes

Prevalence of TB cases in the study: $13.0 \%$

\begin{tabular}{ll}
\hline Index tests & Index: Xpert MTB/RIF \\
\hline Target condition and reference standard(s) & Target condition: pulmonary TB \\
& Reference standard for pulmonary TB: LJ
\end{tabular}

Flow and timing

Comparative

Notes

Methodological quality

\begin{tabular}{lll}
\hline Item & $\begin{array}{l}\text { Authors' judge- } \\
\text { ment }\end{array}$ & $\begin{array}{l}\text { Risk of bias } \\
\text { concerns }\end{array}$ \\
\hline
\end{tabular}

\section{DOMAIN 1: Patient Selection}

Was a consecutive or random sample of patients enrolled?

Yes

\begin{tabular}{lc}
\hline Was a case-control design avoided? & Yes \\
\hline Did the study avoid inappropriate exclusions? & Yes \\
\hline & Low Low L L L L
\end{tabular}

DOMAIN 2: Index Test Xpert MTB/RIF

Were the index test results interpreted without knowledge of the results of Yes the reference standard?

If a threshold was used, was it pre-specified?

Yes

\begin{tabular}{lcc}
\hline Low & Low \\
\hline DOMAIN 3: Reference Standard & Low \\
\hline Is the reference standards likely to correctly classify the target condition? $\quad$ Yes &
\end{tabular}

Were the reference standard results for TB detection interpreted without Unclear knowledge of the results of the index test?

Were the reference standard results for rifampicin resistance detection in-

terpreted without knowledge of the results of the index test?

Unclear

High

\section{DOMAIN 4: Flow and Timing}

Was there an appropriate interval between index test and reference stan- Yes dard? 
Mollel 2017 (Continued)

Did all patients receive the same reference standard?

Yes

Were all patients included in the analysis?

Yes

Low

Moure 2011

\section{Study characteristics}

Patient sampling

Patient characteristics and setting
Cross-sectional design, enrolment by convenience, retrospective data collection

Presenting signs and symptoms: not reported; participants found to be smear-negative on microscopy

Age: older than 15 years; mean: 42 years

Sex, female: not reported

HIV infection: not reported

History of TB: not reported

Sample size: 107

Clinical setting: laboratory-based

Laboratory level: central

Country: Spain

World Bank Income Classification: high income

High TB burden country: no

High MDR-TB burden country: no

High TB/HIV burden country: no

TB incidence rate: 15 per 100,000

MDR-TB prevalence: percentage MDR-TB among new TB cases $=0.2 \%$

(Source: Survey in Galicia region, 2005) and among retreatment cases =

$1.5 \%$ (Source: Survey in Galicia region, 2005)

Prevalence of TB cases in the study: $72.9 \%$

Index tests Index: Xpert MTB/RIF

Target condition and reference standard(s)

Target condition: pulmonary TB

Reference standard for pulmonary TB: LJ culture and MGIT 960

Flow and timing

\section{Comparative}

Sample set included 1 pulmonary biopsy specimen 
Moure 2011 (Continued)

Of 85 pulmonary and extrapulmonary specimens tested, 6 were positive by Xpert MTB/RIF for rifampicin resistance, and 7 specimens were positive by the reference standard

\section{Methodological quality}

\begin{tabular}{llll}
\hline Item Authors' judgement & Risk of bias $\begin{array}{l}\text { Applicability con- } \\
\text { cerns }\end{array}$ \\
\hline
\end{tabular}

\section{DOMAIN 1: Patient Selection}

Was a consecutive or random sample of patients en- No rolled?

\begin{tabular}{llll}
\hline Was a case-control design avoided? & Yes & High & Yes \\
\hline Did the study avoid inappropriate exclusions? & & \\
\hline DOMAIN 2: Index Test Xpert MTB/RIF & Yes & Les \\
\hline $\begin{array}{l}\text { Were the index test results interpreted without knowl- } \\
\text { edge of the results of the reference standard? }\end{array}$ & & Unclear \\
\hline If a threshold was used, was it pre-specified? & & Low \\
\hline
\end{tabular}

\section{DOMAIN 3: Reference Standard}

Is the reference standards likely to correctly classify the Yes target condition?

Were the reference standard results for TB detection in- Yes terpreted without knowledge of the results of the index test?

Were the reference standard results for rifampicin resistance detection interpreted without knowledge of the results of the index test?

\begin{tabular}{llll}
\hline LOMAIN 4: Flow and Timing & Low & \\
\hline $\begin{array}{l}\text { Was there an appropriate interval between index test } \\
\text { and reference standard? }\end{array}$ & Yes \\
\hline Did all patients receive the same reference standard? & Yes \\
\hline Were all patients included in the analysis? & Yes \\
\hline
\end{tabular}


Moussa 2016

\section{Study characteristics}

Patient sampling

Cross-sectional design, unknown manner of enrolment, prospective data collection

Patient characteristics and setting

Presenting signs and symptoms: clinical signs of pulmonary TB

Age: 18 to 60 years

Sex, female: not reported

HIV infection: $0 \%$

History of TB: not reported

Sample size: 218

Clinical setting: laboratory-based

Laboratory level: central

Country: Egypt

World Bank Income Classification: middle income

High TB burden country: no

High MDR-TB burden country: no

High TB/HIV burden country: no

Prevalence of TB cases in the study: $32.1 \%$

\begin{tabular}{ll}
\hline Index tests $\quad$ Index: Xpert MTB/RIF &
\end{tabular}

Target condition and reference standard(s)

Target condition: pulmonary TB

Reference standard for pulmonary TB: LJ

Target condition: rifampicin resistance

Reference standard for rifampicin resistance: Middlebrook $7 \mathrm{H} 11$ agar

\section{Flow and timing}

\section{Comparative}

\section{Notes}

Methodological quality

\begin{tabular}{lll}
\hline Item & $\begin{array}{l}\text { Authors' judge- } \\
\text { ment }\end{array}$ & $\begin{array}{c}\text { Risk of bias } \\
\text { Applicability } \\
\text { concerns }\end{array}$ \\
\hline DOMAIN 1: Patient Selection & Unclear \\
\hline Was a consecutive or random sample of patients enrolled? & Yes \\
\hline Was a case-control design avoided? & \\
\hline
\end{tabular}


Moussa 2016 (Continued)

\begin{tabular}{lll}
\hline & Unclear & Unclear \\
\hline
\end{tabular}

\section{DOMAIN 2: Index Test Xpert MTB/RIF}

Were the index test results interpreted without knowledge of the re- Yes sults of the reference standard?

If a threshold was used, was it pre-specified? Yes

\begin{tabular}{|c|c|c|}
\hline & Low & Low \\
\hline \multicolumn{3}{|l|}{ DOMAIN 3: Reference Standard } \\
\hline $\begin{array}{l}\text { Is the reference standards likely to correctly classify the target condi- } \\
\text { tion? }\end{array}$ & Yes & \\
\hline $\begin{array}{l}\text { Were the reference standard results for TB detection interpreted } \\
\text { without knowledge of the results of the index test? }\end{array}$ & Yes & \\
\hline \multirow[t]{2}{*}{$\begin{array}{l}\text { Were the reference standard results for rifampicin resistance detec- } \\
\text { tion interpreted without knowledge of the results of the index test? }\end{array}$} & Yes & \\
\hline & Low & Low \\
\hline
\end{tabular}

DOMAIN 4: Flow and Timing

Was there an appropriate interval between index test and reference Yes standard?

\begin{tabular}{lc}
\hline Did all patients receive the same reference standard? & Yes \\
\hline Were all patients included in the analysis? & Low \\
\hline
\end{tabular}

\section{Mutingwende 2015}

\section{Study characteristics}

\begin{tabular}{ll}
\hline Patient sampling & $\begin{array}{l}\text { Cross-sectional design, unknown manner of enrolment, } \\
\text { prospective data collection }\end{array}$ \\
\hline Patient characteristics and setting & $\begin{array}{l}\text { Age: median } 46 \text { years (IQR } 39 \text { to } 51) \\
\text { Sex, female: } 4 \%\end{array}$ \\
HIV infection: $74 \%$ & History of TB: $57 \%$ \\
Sample size: 306 & Clinical setting: outpatient \\
\hline
\end{tabular}


Laboratory level: central

Country: South Africa

World Bank Income Classification: middle income

High TB burden country: yes

High MDR-TB burden country: yes

High TB/HIV burden country: yes

Prevalence of TB cases in the study: $75.7 \%$

\begin{tabular}{ll}
\hline Index tests & Index: Xpert MTB/RIF \\
\hline Target condition and reference standard(s) & Target condition: pulmonary TB \\
& Reference standard for pulmonary TB: MGIT 960 \\
\hline Flow and timing & $\begin{array}{l}242 \text { test results were missing for Xpert, microscopy and } \\
\text { MGIT }\end{array}$ \\
\hline
\end{tabular}

\section{Comparative}

\section{Notes}

\section{Methodological quality}

\begin{tabular}{lll}
\hline Item & $\begin{array}{l}\text { Authors' judge- } \\
\text { ment }\end{array}$ & $\begin{array}{l}\text { Risk of bias } \\
\text { concerns }\end{array}$ \\
\hline DOMAIN 1: Patient Selection & Unclear \\
\hline Was a consecutive or random sample of patients enrolled? & Yes & Yes \\
\hline Was a case-control design avoided? & & Unclear \\
\hline Did the study avoid inappropriate exclusions? & Low
\end{tabular}

\section{DOMAIN 2: Index Test Xpert MTB/RIF}

Were the index test results interpreted without knowledge of the re- Yes sults of the reference standard?

\begin{tabular}{llll}
\hline & Low & Unclear \\
\hline
\end{tabular}

\section{DOMAIN 3: Reference Standard}

Is the reference standards likely to correctly classify the target condi- Yes tion?

Were the reference standard results for TB detection interpreted with- Yes out knowledge of the results of the index test? 
Mutingwende 2015 (Continued)

Were the reference standard results for rifampicin resistance detection interpreted without knowledge of the results of the index test?

Low

Low

\section{DOMAIN 4: Flow and Timing}

Was there an appropriate interval between index test and reference Yes standard?

Did all patients receive the same reference standard? Yes

Were all patients included in the analysis?

No

\section{High}

\section{N'Guessan 2016}

\section{Study characteristics}

Patient sampling

Cross-sectional design, consecutive enrolment, prospective data collection

Patient characteristics and setting

Presenting signs and symptoms: presumed pulmonary

TB, smear-positive (failure, relapse, default)

Age: mean 33 years (SD 11), range 15 to 73 years

Sex, female: $32 \%$

HIV infection: $18 \%$

History of TB: $100 \%$

Sample size: 63

Clinical setting: not reported

Laboratory level: central

Country: Cote d'Ivoire

World Bank Income Classification: middle income

High TB burden country: no

High MDR-TB burden country: no

High TB/HIV burden country: no 
N'Guessan 2016 (Continued)

Comparative

$$
\text { Notes }
$$

\section{Methodological quality}

\begin{tabular}{lll}
\hline Item & $\begin{array}{l}\text { Authors' judge- } \\
\text { ment }\end{array}$ & $\begin{array}{c}\text { Risk of bias } \\
\text { Applicability } \\
\text { concerns }\end{array}$ \\
\hline DOMAIN 1: Patient Selection & Yes \\
\hline Was a consecutive or random sample of patients enrolled? & Yes & No \\
\hline Was a case-control design avoided? & & High \\
\hline Did the study avoid inappropriate exclusions?
\end{tabular}

DOMAIN 2: Index Test Xpert MTB/RIF

Were the index test results interpreted without knowledge of the results Yes of the reference standard?

If a threshold was used, was it pre-specified?

Yes

\begin{tabular}{l}
\hline Low Low \\
\hline DOMAIN 3: Reference Standard \\
\hline Is the reference standards likely to correctly classify the target condition? Yes \\
\hline $\begin{array}{l}\text { Were the reference standard results for TB detection interpreted without } \\
\text { knowledge of the results of the index test? }\end{array}$ \\
$\begin{array}{l}\text { Were the reference standard results for rifampicin resistance detection } \\
\text { interpreted without knowledge of the results of the index test? }\end{array}$ \\
\hline $\begin{array}{l}\text { DomalN 4: Flow and Timing } \\
\text { Ward? }\end{array}$ \\
\hline \begin{tabular}{l} 
Did all patients receive the same reference standard? \\
\hline Were all patients included in the analysis?
\end{tabular} \\
\hline
\end{tabular}

Low

\section{Ngabonziza 2016}

\section{Study characteristics}


Ngabonziza 2016 (Continued)

Patient sampling

Patient characteristics and setting
Cross-sectional design with consecutive enrolment of participants, prospective data collection

Presenting signs and symptoms: people with presumptive TB

Age: 15 years and older, median 37 years (IQR 28 to 50)

Sex, female: $38 \%$

HIV infection: $27 \%$

History of TB: not reported

Sample size: 600

Clinical setting: outpatient

Laboratory level: central

Country: Rwanda

World Bank Income Classification: low income

High TB burden country: no

High MDR-TB burden country: no

High TB/HIV burden country: no

Prevalence of TB cases in the study: $16.0 \%$

Index tests
Index: Xpert MTB/RIF

Target condition: pulmonary TB

Reference standard for pulmonary TB: LJ and MGIT 960

Flow and timing

Comparative

Notes

\section{Methodological quality}

\begin{tabular}{|c|c|c|c|}
\hline Item & $\begin{array}{l}\text { Authors' judge- } \\
\text { ment }\end{array}$ & Risk of bias & $\begin{array}{l}\text { Applicability } \\
\text { concerns }\end{array}$ \\
\hline
\end{tabular}

\section{DOMAIN 1: Patient Selection}

\begin{tabular}{lcc}
\hline Was a consecutive or random sample of patients enrolled? & Yes & \\
\hline Was a case-control design avoided? & Yes & Yes \\
\hline Did the study avoid inappropriate exclusions? & Low Low
\end{tabular}

DOMAIN 2: Index Test Xpert MTB/RIF 
Ngabonziza 2016 (Continued)

Were the index test results interpreted without knowledge of the results Yes of the reference standard?

If a threshold was used, was it pre-specified?

Yes

\begin{tabular}{lll}
\hline Low & Low & Low \\
\hline
\end{tabular}

\section{DOMAIN 3: Reference Standard}

Is the reference standards likely to correctly classify the target condi- $\quad$ Yes tion?

Were the reference standard results for TB detection interpreted with- Unclear out knowledge of the results of the index test?

Were the reference standard results for rifampicin resistance detection

interpreted without knowledge of the results of the index test?

\begin{tabular}{lll}
\hline & Unclear & Low \\
\hline DOMAIN 4: Flow and Timing & &
\end{tabular}

Was there an appropriate interval between index test and reference Yes standard?

\begin{tabular}{ll}
\hline Did all patients receive the same reference standard? Yes & Y
\end{tabular}

Were all patients included in the analysis? Yes

Low

\section{Nikam 2014}

\section{Study characteristics}

\section{Patient sampling}

Patient characteristics and setting
Cross-sectional design, consecutive enrolment, prospective data collection

Presenting signs and symptoms: symptoms of pulmonary TB

Age: 15 years and older

Sex, female: not reported

HIV infection: not reported

History of TB: not reported

Sample size: 274

Clinical setting: laboratory-based

Laboratory level: central

Country: India

World Bank Income Classification: middle income 
Nikam 2014 (Continued)

High TB burden country: yes

High MDR-TB burden country: yes

High TB/HIV burden country: yes

Prevalence of TB cases in the study: $55.1 \%$

\begin{tabular}{ll}
\hline Index tests & Index: Xpert MTB/RIF \\
\hline Target condition and reference standard(s) & Target condition: pulmonary TB \\
& Reference standard for pulmonary TB: MGIT 960
\end{tabular}

Flow and timing

Comparative

\begin{tabular}{ll}
\hline Notes & $\begin{array}{l}\text { The authors thought that the study may have included par- } \\
\text { ticipants on anti-TB treatment }\end{array}$
\end{tabular}

ticipants on anti-TB treatment

\section{Methodological quality}

\begin{tabular}{lll}
\hline Item & $\begin{array}{l}\text { Authors' judge- } \\
\text { ment }\end{array}$ & $\begin{array}{l}\text { Risk of bias } \\
\text { Applicability } \\
\text { concerns }\end{array}$ \\
\hline
\end{tabular}

\section{DOMAIN 1: Patient Selection}

\begin{tabular}{lccl}
\hline Was a consecutive or random sample of patients enrolled? & Yes & Yes & \\
\hline Was a case-control design avoided? & Yes & Low Unclear \\
\hline Did the study avoid inappropriate exclusions? & & Un \\
\hline
\end{tabular}

\section{DOMAIN 2: Index Test Xpert MTB/RIF}

Were the index test results interpreted without knowledge of the re- Yes

sults of the reference standard?

If a threshold was used, was it pre-specified?

Yes

\begin{tabular}{llll}
\hline & Low & Low \\
\hline DOMAIN 3: Reference Standard &
\end{tabular}

\section{DOMAIN 3: Reference Standard}

Is the reference standards likely to correctly classify the target condi- Yes tion?

Were the reference standard results for TB detection interpreted with- Yes out knowledge of the results of the index test?

Were the reference standard results for rifampicin resistance detection interpreted without knowledge of the results of the index test? 
Nikam 2014 (Continued)

DOMAIN 4: Flow and Timing

Was there an appropriate interval between index test and reference Yes standard?

Did all patients receive the same reference standard? Yes

Were all patients included in the analysis? Yes

Low

\section{Nliwasa 2016}

\section{Study characteristics}

\begin{tabular}{|c|c|}
\hline Patient sampling & $\begin{array}{l}\text { Cross-sectional design, consecutive enrolment, } \\
\text { prospective data collection }\end{array}$ \\
\hline \multirow[t]{14}{*}{ Patient characteristics and setting } & Presenting signs and symptoms: cough for $>2$ weeks \\
\hline & Age: 15 years and older, median: 32 years (IQR 25 to 41 ) \\
\hline & Sex, female: $44 \%$ \\
\hline & HIV infection: $44 \%$ \\
\hline & History of TB: not reported \\
\hline & Sample size: 273 \\
\hline & Clinical setting: outpatient \\
\hline & Laboratory level: central \\
\hline & Country: Malawi \\
\hline & World Bank Income Classification: low income \\
\hline & High TB burden country: no \\
\hline & High MDR-TB burden country: no \\
\hline & High TB/HIV burden country: yes \\
\hline & Prevalence of TB cases in the study: $17.4 \%$ \\
\hline Index tests & Index: Xpert MTB/RIF \\
\hline \multirow[t]{2}{*}{ Target condition and reference standard(s) } & Target condition: pulmonary TB \\
\hline & Reference standard for pulmonary TB: LJ and MGIT 960 \\
\hline \multicolumn{2}{|l|}{ Flow and timing } \\
\hline \multicolumn{2}{|l|}{ Comparative } \\
\hline
\end{tabular}


Nliwasa 2016 (Continued)

Methodological quality

\begin{tabular}{lll}
\hline Item & $\begin{array}{l}\text { Authors' judge- } \\
\text { ment }\end{array}$ & $\begin{array}{c}\text { Risk of bias } \\
\text { Concerns }\end{array}$ \\
\hline DOMAIN 1: Patient Selection & Yes \\
\hline Was a consecutive or random sample of patients enrolled? & Yes & Yes \\
\hline Was a case-control design avoided? & & Low \\
\hline Did the study avoid inappropriate exclusions?
\end{tabular}

DOMAIN 2: Index Test Xpert MTB/RIF

Were the index test results interpreted without knowledge of the results of Yes the reference standard?

If a threshold was used, was it pre-specified? Yes

Low

Low

\section{DOMAIN 3: Reference Standard}

Is the reference standards likely to correctly classify the target condition? Yes

Were the reference standard results for TB detection interpreted without Yes

knowledge of the results of the index test?

Were the reference standard results for rifampicin resistance detection in-

terpreted without knowledge of the results of the index test?

\begin{tabular}{lll}
\hline DOMAIN 4: Flow and Timing & Low & \\
\hline Was there an appropriate interval between index test and reference stan- & Yes \\
dard? & Yes \\
\hline Did all patients receive the same reference standard? & Yes \\
\hline Were all patients included in the analysis?
\end{tabular}

Low

Nosova 2013a

\section{Study characteristics}

\section{Patient sampling}

Cross-sectional design, unknown manner of enrolment, prospective data collection 
Age: adults

Sex, female: not reported

HIV infection: not reported

History of TB: not reported

Sample size: 278

Clinical setting: laboratory-based

Laboratory level: central

Country: Russia

World Bank Income Classification: middle income

High TB burden country: yes

High MDR-TB burden country: yes

High TB/HIV burden country: no

Prevalence of TB cases in the study: $37.2 \%$

Index: Xpert MTB/RIF

Target condition and reference standard(s)

Target condition: pulmonary TB

Reference standard for pulmonary TB: MGIT 960

Reference standard for rifampicin resistance detection: MGIT 960

Flow and timing

Comparative

\section{Notes}

\section{Methodological quality}

\begin{tabular}{lll}
\hline Item & $\begin{array}{l}\text { Authors' judge- } \\
\text { ment }\end{array}$ & $\begin{array}{l}\text { Risk of bias } \\
\text { Applicability } \\
\text { concerns }\end{array}$ \\
\hline
\end{tabular}

\section{DOMAIN 1: Patient Selection}

\begin{tabular}{lccl}
\hline Was a consecutive or random sample of patients enrolled? & Unclear & \\
\hline Was a case-control design avoided? & Yes & \\
\hline Did the study avoid inappropriate exclusions? & Yes & Unclear & Unclear \\
\hline
\end{tabular}

DOMAIN 2: Index Test Xpert MTB/RIF

Were the index test results interpreted without knowledge of the results Yes

of the reference standard? 
Nosova 2013a (Continued)

\section{DOMAIN 3: Reference Standard}

Is the reference standards likely to correctly classify the target condi- Yes tion?

Were the reference standard results for TB detection interpreted with- Yes out knowledge of the results of the index test?

Were the reference standard results for rifampicin resistance detection Yes interpreted without knowledge of the results of the index test?

Low Low

\section{DOMAIN 4: Flow and Timing}

Was there an appropriate interval between index test and reference Yes standard?

Did all patients receive the same reference standard? Yes

Were all patients included in the analysis? Yes

Low

\section{O'Donnell 2015}

\section{Study characteristics}

Patient sampling

Cross-sectional design, consecutive enrolment, prospective data collection

\section{Patient characteristics and setting}

Presenting signs and symptoms: presumed pulmonary TB

Age: median 33 years, range 18 to 63 years

Sex, female: $47 \%$

HIV infection: $51 \%$

History of TB: $28 \%$

Sample size: 173

Clinical setting: outpatient and inpatient

Laboratory level: central

Country: South Africa

World Bank Income Classification: middle income

High TB burden country: yes

High MDR-TB burden country: yes 
O'Donnell 2015 (Continued)

High TB/HIV burden country: yes

Prevalence of TB cases in the study: $76.8 \%$

\begin{tabular}{ll}
\hline Index tests & Index: Xpert MTB/RIF \\
\hline Target condition and reference standard(s) & Target condition: pulmonary TB \\
& $\begin{array}{l}\text { Reference standard for pulmonary TB: } 7 \mathrm{H} 10 \text { agar plates and } \\
\text { MGIT } 960\end{array}$ \\
& $\begin{array}{l}\text { Target condition: rifampicin resistance } \\
\text { Reference standard for rifampicin resistance: } 7 \mathrm{H} 10\end{array}$
\end{tabular}

Flow and timing

Comparative

Notes

\section{Methodological quality}

\begin{tabular}{|c|c|c|c|}
\hline Item & $\begin{array}{l}\text { Authors' judge- } \\
\text { ment }\end{array}$ & Risk of bias & $\begin{array}{l}\text { Applicability } \\
\text { concerns }\end{array}$ \\
\hline
\end{tabular}

\section{DOMAIN 1: Patient Selection}

\begin{tabular}{lccl}
\hline Was a consecutive or random sample of patients enrolled? & Yes & Yes & \\
\hline Was a case-control design avoided? & Yes & Low Low \\
\hline Did the study avoid inappropriate exclusions? & & Low \\
\hline
\end{tabular}

\section{DOMAIN 2: Index Test Xpert MTB/RIF}

Were the index test results interpreted without knowledge of the re- Yes

sults of the reference standard?

If a threshold was used, was it pre-specified?

Yes

\begin{tabular}{lcl}
\hline & Low & Low \\
\hline DOMAIN 3: Reference Standard &
\end{tabular}

\section{DOMAIN 3: Reference Standard}

Is the reference standards likely to correctly classify the target condi- Yes tion?

Were the reference standard results for TB detection interpreted without knowledge of the results of the index test?

Were the reference standard results for rifampicin resistance detection interpreted without knowledge of the results of the index test?

\begin{tabular}{l} 
Low \\
Low \\
\hline
\end{tabular}


O'Donnell 2015 (Continued)

DOMAIN 4: Flow and Timing

Was there an appropriate interval between index test and reference Yes standard?

\begin{tabular}{lc}
\hline Did all patients receive the same reference standard? & Yes \\
\hline Were all patients included in the analysis? & Yes \\
\hline
\end{tabular}

\section{Park 2013}

\section{Study characteristics}

\begin{tabular}{|c|c|}
\hline Patient sampling & $\begin{array}{l}\text { Cross-sectional design, consecutive enrolment, prospective } \\
\text { data collection }\end{array}$ \\
\hline \multirow[t]{14}{*}{ Patient characteristics and setting } & Presenting signs and symptoms: presumed pulmonary TB \\
\hline & Age: 15 years and older \\
\hline & Sex, female: not reported \\
\hline & HIV infection: not reported \\
\hline & History of TB: not reported \\
\hline & Sample size: 320 \\
\hline & Clinical setting: not reported \\
\hline & Laboratory level: central \\
\hline & Country: Republic of Korea \\
\hline & World Bank Income Classification: high income \\
\hline & High TB burden country: no \\
\hline & High MDR-TB burden country: no \\
\hline & High TB/HIV burden country: no \\
\hline & Prevalence of TB cases in the study: $7.2 \%$ \\
\hline Index tests & Index: Xpert MTB/RIF \\
\hline \multirow[t]{4}{*}{ Target condition and reference standard(s) } & Target condition: pulmonary TB \\
\hline & Reference standard for pulmonary TB: LJ and MGIT 960 \\
\hline & Target condition: rifampicin resistance \\
\hline & $\begin{array}{l}\text { Reference standard for rifampicin resistance: LJ and MGIT } \\
960\end{array}$ \\
\hline
\end{tabular}

Flow and timing 
Park 2013 (Continued)

Comparative

$$
\text { Notes }
$$

\section{Methodological quality}

\begin{tabular}{lll}
\hline Item & $\begin{array}{l}\text { Authors' judge- } \\
\text { ment }\end{array}$ & $\begin{array}{l}\text { Risk of bias } \\
\begin{array}{l}\text { Applicability con- } \\
\text { cerns }\end{array}\end{array}$ \\
\hline
\end{tabular}

\section{DOMAIN 1: Patient Selection}

\begin{tabular}{lcc}
\hline Was a consecutive or random sample of patients enrolled? & Yes & \\
\hline Was a case-control design avoided? & Yes & Yes \\
\hline Did the study avoid inappropriate exclusions? & Low Unclear
\end{tabular}

DOMAIN 2: Index Test Xpert MTB/RIF

Were the index test results interpreted without knowledge of the re- Yes sults of the reference standard?

If a threshold was used, was it pre-specified?

Yes

\begin{tabular}{lll}
\hline Low & Low \\
\hline
\end{tabular}

\section{DOMAIN 3: Reference Standard}

Is the reference standards likely to correctly classify the target condi- Yes tion?

Were the reference standard results for TB detection interpreted Yes without knowledge of the results of the index test?

Were the reference standard results for rifampicin resistance detec- Yes tion interpreted without knowledge of the results of the index test?

\section{DOMAIN 4: Flow and Timing}

Was there an appropriate interval between index test and reference Yes standard?

Did all patients receive the same reference standard?

Were all patients included in the analysis?
Yes

Yes 
Pimkina 2015

\section{Study characteristics}

Patient sampling

Cross-sectional design, unknown manner of enrolment, retrospective data collection

Patient characteristics and setting

Presenting signs and symptoms: people with known risk factors for MDR-TB and all retreatment patients including those with extensive lung damage, e.g. cavities

Age: 18 years and older; median 50 years

Sex, female: $29 \%$

HIV infection: not reported

History of TB: $100 \%$

Sample size: 792

Clinical setting: laboratory-based, specimens submitted from local general practitioners and hospitals

Laboratory level: central

Country: Lithuania

World Bank Income Classification: high income

High TB burden country: no

High MDR-TB burden country: no

High TB/HIV burden country: no

Prevalence of TB cases in the study: $48.2 \%$

\begin{tabular}{ll}
\hline Index tests & Index: Xpert MTB/RIF \\
\hline Target condition and reference standard(s) & Target condition: pulmonary TB \\
& Reference standard for pulmonary TB: LJ and MGIT 950 \\
& Target condition: rifampicin resistance \\
& Reference standard for rifampicin resistance: LJ and MGIT 960 \\
\hline
\end{tabular}

Flow and timing

Comparative

Notes

\section{Methodological quality}

\begin{tabular}{llll}
\hline Item Authors' judgement & Risk of bias & $\begin{array}{l}\text { Applicability con- } \\
\text { cerns }\end{array}$
\end{tabular}

\section{DOMAIN 1: Patient Selection}

Was a consecutive or random sample of patients enrolled? Unclear 
Pimkina 2015 (Continued)

Was a case-control design avoided?

Yes

Did the study avoid inappropriate exclusions?

No

\begin{tabular}{lcc}
\hline & High & Low \\
\hline DOMAIN 2: Index Test Xpert MTB/RIF &
\end{tabular}

DOMAIN 2: Index Test Xpert MTB/RIF

Were the index test results interpreted without knowledge of Yes

the results of the reference standard?

\begin{tabular}{llll}
\hline If a threshold was used, was it pre-specified? & Yes & \\
\hline Low & Low \\
\hline
\end{tabular}

\section{DOMAIN 3: Reference Standard}

Is the reference standards likely to correctly classify the tar- Yes get condition?

Were the reference standard results for TB detection inter- Unclear preted without knowledge of the results of the index test?

Were the reference standard results for rifampicin resistance Unclear detection interpreted without knowledge of the results of the index test?

Unclear

Low

\section{DOMAIN 4: Flow and Timing}

Was there an appropriate interval between index test and Yes reference standard?

Did all patients receive the same reference standard? Yes

Were all patients included in the analysis? Yes

Low

Pinyopornpanish 2015

\section{Study characteristics}

Patient sampling

Cross-sectional design, consecutive enrolment, prospective data collection

Patient characteristics and setting

Presenting signs and symptoms: 2 or more of the following symptoms: fever, chronic cough, weight loss, pleuritic chest pain, haemoptysis, and with or without abnormal chest radiograph compatible with pulmonary tuberculosis (e.g. cavitary lesion, infiltration, and miliary pattern)

Age: 15 years and older, mean 56 years (SD 20)

Sex, female: $40 \%$

Xpert MTB/RIF and Xpert MTB/RIF Ultra for pulmonary tuberculosis and rifampicin resistance in adults (Review) 


\author{
HIV infection: $26 \%$ \\ History of TB: not reported \\ Sample size: 109 \\ Clinical setting: not reported \\ Laboratory level: central \\ Country: Thailand
}

World Bank Income Classification: middle income

High TB burden country: yes

High MDR-TB burden country: yes

High TB/HIV burden country: yes

Prevalence of TB cases in the study: $39.4 \%$

\begin{tabular}{ll}
\hline Index tests & Index: Xpert MTB/RIF \\
\hline Target condition and reference standard(s) & Target condition: pulmonary TB \\
& Reference standard for pulmonary TB: MGIT 960 \\
\hline
\end{tabular}

Flow and timing

\section{Comparative}

Notes

\section{Methodological quality}

\begin{tabular}{llll}
\hline Item Authors' judgement & Risk of bias & $\begin{array}{l}\text { Applicability con- } \\
\text { cerns }\end{array}$
\end{tabular}

DOMAIN 1: Patient Selection

\begin{tabular}{llll}
\hline Was a consecutive or random sample of patients enrolled? & Yes \\
\hline Was a case-control design avoided? & Yes & \\
\hline Did the study avoid inappropriate exclusions? & Yes & Low & Unclear \\
\hline
\end{tabular}

\section{DOMAIN 2: Index Test Xpert MTB/RIF}

Were the index test results interpreted without knowledge of

Yes

the results of the reference standard?

If a threshold was used, was it pre-specified?

Yes
Low

\section{DOMAIN 3: Reference Standard}


Pinyopornpanish 2015 (Continued)

Is the reference standards likely to correctly classify the target Yes condition?

Were the reference standard results for TB detection interpret- Yes ed without knowledge of the results of the index test?

Were the reference standard results for rifampicin resistance detection interpreted without knowledge of the results of the index test?

\begin{tabular}{l} 
DOMAIN 4: Flow and Timing \\
\hline $\begin{array}{l}\text { Was there an appropriate interval between index test and refer- Yes } \\
\text { ence standard? }\end{array}$
\end{tabular}
ence standard?

Did all patients receive the same reference standard?

Yes

Were all patients included in the analysis?

Yes

Low

\section{Rachow 2011}

\section{Study characteristics}

\section{Patient sampling}

Patient characteristics and setting
Cross-sectional design, consecutive enrolment, retrospective data collection

Presenting signs and symptoms: presumptive pulmonary TB based on clinical and radiographic findings

Age: mean 39 years (SD 13.8)

Sex, female: $51.7 \%$

HIV infection: $58.9 \%$

History of TB: not reported

Sample size: 249

Clinical setting: referral hospital

Laboratory level: central

Country: Tanzania

World Bank Income Classification: low income

High TB burden country: yes

High MDR-TB burden country: no

High TB/HIV burden country: yes

TB incidence rate: 169 per 100,000 
Rachow 2011 (Continued)

MDR-TB prevalence: percentage MDR-TB among new TB cases $=1.1 \%$

(Source: nationwide survey, 2007) and among retreatment cases $=0 \%$

(Source: Nationwide survey, 2007)

Prevalence of TB cases in the study: $27.7 \%$

\section{Index tests}

Index: Xpert MTB/RIF

Target condition and reference standard(s)
Target condition: pulmonary TB

Reference standard for pulmonary TB: LJ culture and MGIT 960

Target condition: rifampicin resistance

Reference standard for rifampicin resistance: MGIT 960

Flow and timing

Comparative

Notes Participants were followed for a period of 56 days. Among 77 participants classified as smear-negative, culture-negative 'clinical TB', Xpert MTB/RIF was positive in $7(9.1 \%)$ participants

No participants were found to have rifampicin resistance

\section{Methodological quality}

\begin{tabular}{llll}
\hline Item & Authors' judgement & Risk of bias & $\begin{array}{l}\text { Applicability con- } \\
\text { cerns }\end{array}$ \\
\hline
\end{tabular}

DOMAIN 1: Patient Selection

Was a consecutive or random sample of patients en- Yes rolled?

\begin{tabular}{llll}
\hline Was a case-control design avoided? & Yes & \\
\hline Did the study avoid inappropriate exclusions? & Yes & Unclear \\
\hline
\end{tabular}

\section{DOMAIN 2: Index Test Xpert MTB/RIF}

Were the index test results interpreted without knowl- Yes edge of the results of the reference standard?

If a threshold was used, was it pre-specified? Yes

\begin{tabular}{lll}
\hline Low & Low \\
\hline
\end{tabular}

\section{DOMAIN 3: Reference Standard}

Is the reference standards likely to correctly classify Yes the target condition?

Were the reference standard results for TB detection Yes interpreted without knowledge of the results of the index test? 


\section{Rachow 2011 (Continued)}

Were the reference standard results for rifampicin resistance detection interpreted without knowledge of the results of the index test?

\section{Low}

Low

\section{DOMAIN 4: Flow and Timing}

Was there an appropriate interval between index test Yes and reference standard?

Did all patients receive the same reference standard? Yes

Were all patients included in the analysis? Unclear

Unclear

\section{Reddy 2017}

\section{Study characteristics}

Patient sampling

Cross-sectional design, random enrolment, prospective data collection

Patient characteristics and setting

Presenting signs and symptoms: TB symptoms

Age: 18 to 60 years

Sex, female: $47 \%$

HIV infection: not reported

History of TB: $33 \%$

Sample size: 705

Clinical setting: outpatient

Laboratory level: central

Country: South Africa

World Bank Income Classification: middle income

High TB burden country: yes

High MDR-TB burden country: yes

High TB/HIV burden country: yes

Prevalence of TB cases in the study: $23.8 \%$

Index tests Index: Xpert MTB/RIF

Target condition and reference standard(s)

Target condition: pulmonary TB

Reference standard for pulmonary TB: MGIT 960

Flow and timing

Xpert MTB/RIF and Xpert MTB/RIF Ultra for pulmonary tuberculosis and rifampicin resistance in adults (Review) 
Reddy 2017 (Continued)

Comparative

$$
\text { Notes }
$$

\section{Methodological quality}

\begin{tabular}{lll}
\hline Item & $\begin{array}{l}\text { Authors' judge- } \\
\text { ment }\end{array}$ & $\begin{array}{c}\text { Risk of bias Applicability } \\
\text { concerns }\end{array}$ \\
\hline
\end{tabular}

\section{DOMAIN 1: Patient Selection}

Was a consecutive or random sample of patients enrolled?

Was a case-control design avoided?

Did the study avoid inappropriate exclusions?
Yes

Yes

Yes

Low Low

\section{DOMAIN 2: Index Test Xpert MTB/RIF}

Were the index test results interpreted without knowledge of the results of Yes the reference standard?

If a threshold was used, was it pre-specified?

Yes

\section{Low}

Low

\section{DOMAIN 3: Reference Standard}

Is the reference standards likely to correctly classify the target condition?

Were the reference standard results for TB detection interpreted without Yes knowledge of the results of the index test?

Were the reference standard results for rifampicin resistance detection interpreted without knowledge of the results of the index test?

\begin{tabular}{lll}
\hline & Low & Low \\
\hline DOMAIN 4: Flow and Timing & Yes \\
\hline $\begin{array}{l}\text { Was there an appropriate interval between index test and reference stan- } \\
\text { dard? }\end{array}$ & Yes \\
\hline Did all patients receive the same reference standard? & Yes \\
\hline Were all patients included in the analysis? &
\end{tabular}

Low

\section{Reechaipichitkul 2017}

\section{Study characteristics}




\section{Reechaipichitkul 2017 (Continued)}

Patient sampling

Patient characteristics and setting
Cross-sectional design, unknown manner of enrolment, prospective data collection

Presenting signs and symptoms: clinical signs and symptoms of pulmonary TB, including cough and prolonged

fever of $>2$ weeks

Age: 15 years and older, mean 55 years (SD 18)

Sex, female: $34 \%$

HIV infection: $5 \%$

History of TB: $38 \%$

Sample size: 125

Clinical setting: not reported

Laboratory level: intermediate

Country: Thailand

World Bank Income Classification: middle income

High TB burden country: yes

High MDR-TB burden country: yes

High TB/HIV burden country: yes

Prevalence of TB cases in the study: $50.4 \%$

\begin{tabular}{ll}
\hline Index tests & Index: Xpert MTB/RIF \\
\hline Target condition and reference standard(s) & Target condition: pulmonary TB \\
& Reference standard for pulmonary TB: LJ \\
\hline
\end{tabular}

Flow and timing

Comparative

Notes

\section{Methodological quality}

\begin{tabular}{lll}
\hline Item & $\begin{array}{l}\text { Authors' judge- } \\
\text { ment }\end{array}$ & $\begin{array}{c}\text { Risk of bias } \\
\text { Applicability } \\
\text { concerns }\end{array}$ \\
\hline DOMAIN 1: Patient Selection & Unclear \\
\hline Was a consecutive or random sample of patients enrolled? & Yes & Yes \\
\hline Was a case-control design avoided? & & Unclear \\
\hline Did the study avoid inappropriate exclusions?
\end{tabular}

\section{DOMAIN 2: Index Test Xpert MTB/RIF}

Xpert MTB/RIF and Xpert MTB/RIF Ultra for pulmonary tuberculosis and rifampicin resistance in adults (Review) 
Reechaipichitkul 2017 (Continued)

Were the index test results interpreted without knowledge of the re- Yes sults of the reference standard?

\begin{tabular}{lcc}
\hline If a threshold was used, was it pre-specified? & Yes \\
\hline & Low Unclear
\end{tabular}

\title{
DOMAIN 3: Reference Standard
}

Is the reference standards likely to correctly classify the target condi- Yes tion?

Were the reference standard results for TB detection interpreted with- Unclear out knowledge of the results of the index test?

Were the reference standard results for rifampicin resistance detection interpreted without knowledge of the results of the index test?

\begin{tabular}{lll}
\hline & Unclear & Low \\
\hline DOMAIN 4: Flow and Timing & Yes \\
\hline $\begin{array}{l}\text { Was there an appropriate interval between index test and reference } \\
\text { standard? }\end{array}$ & Yes \\
\hline Did all patients receive the same reference standard? & Yes \\
\hline Were all patients included in the analysis?
\end{tabular}

Low

Rice 2017

\section{Study characteristics}

Patient sampling

Cross-sectional design consecutive enrolment, retrospective data collection

\section{Patient characteristics and setting}

\author{
Presenting signs and symptoms: signs and symptoms of pul- \\ monary TB \\ Age: median 50 years (IQR 35 to 60 ) \\ Sex, female: not reported \\ HIV infection: not reported \\ History of TB: not reported \\ Sample size: 751 \\ Clinical setting: outpatient \\ Laboratory level: central \\ Country: USA \\ World Bank Income Classification: high income
}


Rice 2017 (Continued)

High TB burden country: no

High MDR-TB burden country: no

High TB/HIV burden country: no

Prevalence of TB cases in the study: $18.2 \%$

\begin{tabular}{ll}
\hline Index tests & Index: Xpert MTB/RIF \\
\hline Target condition and reference standard(s) & Target condition: pulmonary TB \\
& $\begin{array}{l}\text { Reference standard for pulmonary TB: Middlebrook solid, MGIT } \\
960\end{array}$ \\
& Target condition: rifampicin resistance \\
& Reference standard for rifampicin resistance: MGIT 960
\end{tabular}

Flow and timing

\section{Comparative}

Notes

Participants were also tested with Xpert if the test result would alter case management or TB control activities

\section{Methodological quality}

\begin{tabular}{llll}
\hline Item & Authors' judgement & Risk of bias & $\begin{array}{l}\text { Applicability con- } \\
\text { cerns }\end{array}$
\end{tabular}

\section{DOMAIN 1: Patient Selection}

\begin{tabular}{lll}
\hline Was a consecutive or random sample of patients enrolled? & Yes \\
\hline Was a case-control design avoided? & Yes \\
\hline Did the study avoid inappropriate exclusions? & Yes & Low \\
\hline
\end{tabular}

DOMAIN 2: Index Test Xpert MTB/RIF

Were the index test results interpreted without knowledge of Yes the results of the reference standard?

If a threshold was used, was it pre-specified? Yes

\begin{tabular}{ll}
\hline DOMAIN 3: Reference Standard & Low \\
\hline $\begin{array}{l}\text { Is the reference standards likely to correctly classify the target } \quad \text { Yes } \\
\text { condition? }\end{array}$ \\
\hline $\begin{array}{l}\text { Were the reference standard results for TB detection interpret- } \\
\text { ed without knowledge of the results of the index test? }\end{array}$ \\
\hline
\end{tabular}


Rice 2017 (Continued)

Were the reference standard results for rifampicin resistance detection interpreted without knowledge of the results of the index test?

Unclear

Low

\section{DOMAIN 4: Flow and Timing}

Was there an appropriate interval between index test and refer- Yes ence standard?

Did all patients receive the same reference standard? Yes

Were all patients included in the analysis? Yes

\section{Safianowska 2012}

\section{Study characteristics}

Patient sampling

Cross-sectional design, consecutive enrolment, prospective data collection

Patient characteristics and setting

Presenting signs and symptoms: presumptive TB

Age: mean 61 years, range 20 to 97 years

Sex, female: $36.6 \%$

HIV infection: $0 \%$

History of TB: not reported

Sample size: 145

Clinical setting: laboratory-based

Laboratory level: intermediate

Country: Poland

World Bank Income Classification: high income

TB incidence rate: 23 per 100,000

MDR-TB prevalence: percentage MDR-TB among new TB cases $=0.5 \%$ (Source: nationwide surveillance, 2011) and among retreatment cases $=3.5 \%$ (Source: nationwide surveillance, 2011)

High TB burden country: no

High MDR-TB burden country: no

High TB/HIV burden country: no

Prevalence of TB cases in the study: $11.8 \%$ Index: Xpert MTB/RIF 


\section{Safianowska 2012 (Continued)}

Target condition and reference standard(s)
Target condition: pulmonary TB

Reference standard for pulmonary TB: LJ culture

Target condition: rifampicin resistance

Reference standard for rifampicin resistance: LJ media, method not specified

\begin{tabular}{llll}
\hline Flow and timing & & \\
\hline Comparative & & & \\
\hline Motes & Authors' judgement & Risk of bias & $\begin{array}{c}\text { Applicability con- } \\
\text { cerns }\end{array}$
\end{tabular}

\section{DOMAIN 1: Patient Selection}

\begin{tabular}{llll}
\hline Was a consecutive or random sample of patients enrolled? & Yes & \\
\hline Was a case-control design avoided? & Yes & \\
\hline Did the study avoid inappropriate exclusions? & Yes & Low & Unclear \\
\hline
\end{tabular}

\section{DOMAIN 2: Index Test Xpert MTB/RIF}

Were the index test results interpreted without knowledge of Yes the results of the reference standard?

\begin{tabular}{ll}
\hline If a threshold was used, was it pre-specified? Yes & Ye
\end{tabular}

\begin{tabular}{lll}
\hline Low & Low \\
\hline
\end{tabular}

\section{DOMAIN 3: Reference Standard}

Is the reference standards likely to correctly classify the tar- Yes get condition?

Were the reference standard results for TB detection inter-

No preted without knowledge of the results of the index test?

Were the reference standard results for rifampicin resistance No detection interpreted without knowledge of the results of the index test?

High

Low

\section{DOMAIN 4: Flow and Timing}

Was there an appropriate interval between index test and Yes reference standard? 
Safianowska 2012 (Continued)

Did all patients receive the same reference standard?

Yes

Were all patients included in the analysis?

Yes

Low

Sah 2017

\section{Study characteristics}

Patient sampling

Cross-sectional design, consecutive enrolment, prospective data collection

\section{Patient characteristics and setting}

Presenting signs and symptoms: presumptive TB

Age: 20 to 83 years

Sex, female: not reported

HIV infection: not reported

History of TB: not reported

Sample size: 105

Clinical setting: not reported

Laboratory level: central

Country: Nepal

World Bank Income Classification: low income

High TB burden country: no

High MDR-TB burden country: no

High TB/HIV burden country: no

Prevalence of TB cases in the study: $37.1 \%$

Index tests Index: Xpert MTB/RIF

Target condition and reference standard(s)

Target condition: pulmonary TB

Reference standard for pulmonary TB: LJ

Target condition: rifampicin resistance

Reference standard for rifampicin resistance: LJ

Flow and timing

Comparative

Notes

\section{Methodological quality}


Sah 2017 (Continued)

\begin{tabular}{|c|c|c|c|}
\hline Item & Authors' judge- & Risk of bias & $\begin{array}{l}\text { Applicability } \\
\text { concerns }\end{array}$ \\
\hline
\end{tabular}

\section{DOMAIN 1: Patient Selection}

\begin{tabular}{lc}
\hline Was a consecutive or random sample of patients enrolled? & Yes \\
\hline Was a case-control design avoided? & Yes \\
\hline Did the study avoid inappropriate exclusions? & Yes
\end{tabular}

\begin{tabular}{llc}
\hline & Low & Unclear \\
\hline DOMAIN 2: Index Test Xpert MTB/RIF & & \\
\hline $\begin{array}{l}\text { Were the index test results interpreted without knowledge of the results } \\
\text { of the reference standard? }\end{array}$ & Yes & Low \\
\hline If a threshold was used, was it pre-specified? & Low \\
\hline
\end{tabular}

\section{DOMAIN 3: Reference Standard}

Is the reference standards likely to correctly classify the target condi- Yes tion?

Were the reference standard results for TB detection interpreted with- Yes out knowledge of the results of the index test?

Were the reference standard results for rifampicin resistance detection Yes interpreted without knowledge of the results of the index test?

\begin{tabular}{lll}
\hline & Low & Low \\
\hline DOMAIN 4: Flow and Timing & Yes \\
\hline $\begin{array}{l}\text { Was there an appropriate interval between index test and reference } \\
\text { standard? }\end{array}$ & Yes \\
\hline Did all patients receive the same reference standard? & Yes \\
\hline Were all patients included in the analysis? &
\end{tabular}

\section{Low}

\section{Scott 2011}

\section{Study characteristics}

Patient sampling

Patient characteristics and setting
Cross-sectional design, consecutive enrolment, prospective data collection

Presenting signs and symptoms: presumptive TB presenting with cough, fever, night sweats, and/or weight loss 
Scott 2011 (Continued)

Age: mean 32 years, range 19 to 75 years

Sex, female: $41.1 \%$

HIV infection: $69.0 \%$

History of TB: not reported

Sample size: 177

Clinical setting: primary care clinic

Laboratory level: central

Country: South Africa, Johannesburg

World Bank Income Classification: middle income

High TB burden country: yes

High MDR-TB burden country: yes

High TB/HIV burden country: yes

TB incidence rate: 993 per 100,000

MDR-TB prevalence: percentage MDR-TB among new TB cases $=1.4 \%$

(Source: survey in Gauteng province, 2002) and among retreatment cases = $5.5 \%$ (Source: survey in Gauteng province, 2002)

Prevalence of TB cases in the study: $37.9 \%$

Index tests Index: Xpert MTB/RIF

Target condition and reference standard(s)

Target condition: pulmonary TB

Reference standard for pulmonary TB: MGIT 960

Target condition: rifampicin resistance

Reference standard for rifampicin resistance: MGIT 960

Flow and timing

\section{Comparative}

Notes

1 follow-up visit was performed approximately 60 days after enrolment

Xpert MTB/RIF was performed on frozen specimens while MGIT culture and smear microscopy were performed on fresh specimens

\section{Methodological quality}

\begin{tabular}{llll}
\hline Item & Authors' judgement & Risk of bias & \multicolumn{1}{c}{$\begin{array}{l}\text { Applicability con- } \\
\text { cerns }\end{array}$}
\end{tabular}

\section{DOMAIN 1: Patient Selection}

Was a consecutive or random sample of patients en- Yes rolled?

Was a case-control design avoided?

Yes

Xpert MTB/RIF and Xpert MTB/RIF Ultra for pulmonary tuberculosis and rifampicin resistance in adults (Review) 
Scott 2011 (Continued)

Did the study avoid inappropriate exclusions?

Yes

\begin{tabular}{lll}
\hline Low & Low \\
\hline
\end{tabular}

DOMAIN 2: Index Test Xpert MTB/RIF

Were the index test results interpreted without knowl- Yes

edge of the results of the reference standard?

If a threshold was used, was it pre-specified? Yes

\begin{tabular}{lll}
\hline Low & Unclear \\
\hline
\end{tabular}

\section{DOMAIN 3: Reference Standard}

Is the reference standards likely to correctly classify Yes

the target condition?

Were the reference standard results for TB detection Yes

interpreted without knowledge of the results of the

index test?

Were the reference standard results for rifampicin resistance detection interpreted without knowledge of

Yes the results of the index test?

Low Low

\section{DOMAIN 4: Flow and Timing}

Was there an appropriate interval between index test Yes and reference standard?

Did all patients receive the same reference standard? Yes

Were all patients included in the analysis? Yes

Low

\section{Scott 2017}

\section{Study characteristics}

Patient sampling

Cross-sectional design, consecutive enrolment, prospective data collection

Patient characteristics and setting

Presenting signs and symptoms: presumptive TB, including presence of a cough for 2 weeks, weight loss, night sweats, fever, chest pain

Age: mean 34 years, range 18 to 60 years

Sex, female: $38 \%$

HIV infection: $73 \%$ 
Scott 2017 (Continued)

History of TB: $15 \%$

Sample size: 206

Clinical setting: outpatient

Laboratory level: central

Country: South Africa

World Bank Income Classification: middle income

High TB burden country: yes

High MDR-TB burden country: yes

High TB/HIV burden country: yes

Prevalence of TB cases in the study: $32.1 \%$

\begin{tabular}{ll}
\hline Index tests & Index: Xpert MTB/RIF \\
\hline Target condition and reference standard(s) & Target condition: pulmonary TB \\
& Reference standard for pulmonary TB: MGIT 960 \\
\hline
\end{tabular}

Flow and timing

\section{Comparative}

Notes

\section{Methodological quality}

\begin{tabular}{lll}
\hline Item & $\begin{array}{l}\text { Authors' judge- } \\
\text { ment }\end{array}$ & $\begin{array}{c}\text { Risk of bias } \\
\text { Applicability } \\
\text { concerns }\end{array}$ \\
\hline DOMAIN 1: Patient Selection & Yes \\
\hline Was a consecutive or random sample of patients enrolled? & Yes & Yes \\
\hline Was a case-control design avoided? & & Low \\
\hline Did the study avoid inappropriate exclusions?
\end{tabular}

\section{DOMAIN 2: Index Test Xpert MTB/RIF}

Were the index test results interpreted without knowledge of the re- Yes sults of the reference standard?

\begin{tabular}{lcc}
\hline If a threshold was used, was it pre-specified? & Yes & Low \\
\hline
\end{tabular}

\section{DOMAIN 3: Reference Standard}

Is the reference standards likely to correctly classify the target condi- Yes tion? 
Scott 2017 (Continued)

Were the reference standard results for TB detection interpreted with- Yes out knowledge of the results of the index test?

Were the reference standard results for rifampicin resistance detection interpreted without knowledge of the results of the index test?

\begin{tabular}{lll}
\hline & Low & Low \\
\hline DOMAIN 4: Flow and Timing & Yes \\
\hline $\begin{array}{l}\text { Was there an appropriate interval between index test and reference } \\
\text { standard? }\end{array}$ & Yes Yes \\
\hline Did all patients receive the same reference standard? & Low \\
\hline Were all patients included in the analysis? &
\end{tabular}

\section{Shao 2017}

\section{Study characteristics}

Patient sampling

Cross-sectional design, unknown manner of enrolment, prospective data collection

Patient characteristics and setting

Presenting signs and symptoms: presumptive TB

Age: mean 53 years (SD 19)

Sex, female: $31 \%$

HIV infection: not reported

History of TB: not reported

Sample size: 225

Clinical setting: outpatient

Laboratory level: peripheral

Country: China

World Bank Income Classification: middle income

High TB burden country: yes

High MDR-TB burden country: yes

High TB/HIV burden country: yes

Prevalence of TB cases in the study: $38.1 \%$

\begin{tabular}{ll}
\hline Index tests & Index: Xpert MTB/RIF \\
\hline Target condition and reference standard(s) & Target condition: pulmonary TB \\
& Reference standard for pulmonary TB: LJ
\end{tabular}

Xpert MTB/RIF and Xpert MTB/RIF Ultra for pulmonary tuberculosis and rifampicin resistance in adults (Review) 
Shao 2017 (Continued)

Flow and timing

129 presumed TB patients were excluded

\section{Comparative}

Notes

\section{Methodological quality}

\begin{tabular}{|c|c|c|c|}
\hline Item & $\begin{array}{l}\text { Authors' judge- } \\
\text { ment }\end{array}$ & Risk of bias & $\begin{array}{l}\text { Applicability } \\
\text { concerns }\end{array}$ \\
\hline
\end{tabular}

\section{DOMAIN 1: Patient Selection}

\begin{tabular}{lcc}
\hline Was a consecutive or random sample of patients enrolled? & Unclear \\
\hline Was a case-control design avoided? & Yes & Yes \\
\hline Did the study avoid inappropriate exclusions? & Unclear L Low \\
\hline
\end{tabular}

\section{DOMAIN 2: Index Test Xpert MTB/RIF}

Were the index test results interpreted without knowledge of the results Yes

of the reference standard?

If a threshold was used, was it pre-specified?

Yes

Low

Low

\section{DOMAIN 3: Reference Standard}

Is the reference standards likely to correctly classify the target condition? Yes

Were the reference standard results for TB detection interpreted without Yes

knowledge of the results of the index test?

Were the reference standard results for rifampicin resistance detection

interpreted without knowledge of the results of the index test?

\begin{tabular}{lll}
\hline & Low & Low \\
\hline DOMAIN 4: Flow and Timing & Yes \\
\hline $\begin{array}{l}\text { Was there an appropriate interval between index test and reference stan- } \\
\text { dard? }\end{array}$ & No & High \\
\hline Did all patients receive the same reference standard? & & \\
\hline Were all patients included in the analysis?
\end{tabular}


Sharma 2015

\section{Study characteristics}

Patient sampling

Cross-sectional design, consecutive enrolment, prospective data collection

Patient characteristics and setting

Presenting signs and symptoms: clinical suspicion of TB

Age: adults, mean 37 years (SD 18)

Sex, female: $35 \%$

HIV infection: not reported

History of TB: not reported

Sample size: 1437

Clinical setting: laboratory-based

Laboratory level: central

Country: India

World Bank Income Classification: middle income

High TB burden country: yes

High MDR-TB burden country: yes

High TB/HIV burden country: yes

Prevalence of TB cases in the study: $31.2 \%$

\begin{tabular}{ll}
\hline Index tests $\quad$ Index: Xpert MTB/RIF &
\end{tabular}

Target condition and reference standard(s)

Target condition: pulmonary TB

Reference standard for pulmonary TB: LJ and MGIT 960

Target condition: rifampicin resistance

Reference standard for rifampicin resistance: LJ

Flow and timing

Comparative

Notes

\section{Methodological quality}

\begin{tabular}{lll}
\hline Item & $\begin{array}{l}\text { Authors' judge- } \\
\text { ment }\end{array}$ & $\begin{array}{l}\text { Risk of bias } \\
\text { Applicability } \\
\text { concerns }\end{array}$ \\
\hline
\end{tabular}

\section{DOMAIN 1: Patient Selection}

\begin{tabular}{lc}
\hline Was a consecutive or random sample of patients enrolled? & Yes \\
\hline Was a case-control design avoided? & Yes \\
\hline Did the study avoid inappropriate exclusions? & \\
\hline
\end{tabular}


Sharma 2015 (Continued)

Low

Unclear

\section{DOMAIN 2: Index Test Xpert MTB/RIF}

Were the index test results interpreted without knowledge of the re- Yes sults of the reference standard?

\begin{tabular}{lcl}
\hline If a threshold was used, was it pre-specified? & Yes \\
\hline Low Low
\end{tabular}

\section{DOMAIN 3: Reference Standard}

Is the reference standards likely to correctly classify the target condi- Yes tion?

Were the reference standard results for TB detection interpreted with- Yes out knowledge of the results of the index test?

Were the reference standard results for rifampicin resistance detec- Yes tion interpreted without knowledge of the results of the index test?

\begin{tabular}{lll}
\hline LOMAIN 4: Flow and Timing & Low \\
\hline $\begin{array}{l}\text { Was there an appropriate interval between index test and reference } \\
\text { standard? }\end{array}$ & Yes \\
\hline Did all patients receive the same reference standard? & Yes \\
\hline Were all patients included in the analysis?
\end{tabular}

Low

\section{Shenai 2016}

\section{Study characteristics}

\section{Patient sampling}

Cross-sectional design, unknown manner of enrolment, prospective data collection

\section{Patient characteristics and setting}


Shenai 2016 (Continued)

Laboratory level: central

Country: Brazil, South Africa, Uganda

World Bank Income Classification: low and middle income

High TB burden country: yes (Brazil), yes (South Africa), no (Ugan-

da)

High MDR-TB burden country: no (Brazil), yes (South Africa), no (Uganda)

High TB/HIV burden country: yes (Brazil), yes (South Africa), yes (Uganda)

Prevalence of TB cases in the study: $28.9 \%$

\begin{tabular}{|c|c|c|}
\hline Index tests & Index: Xpert MTB/RIF & \\
\hline \multirow[t]{2}{*}{ Target condition and reference standard(s) } & \multicolumn{2}{|l|}{ Target condition: pulmonary TB } \\
\hline & \multicolumn{2}{|c|}{ Reference standard for pulmonary TB: LJ and MGIT 960} \\
\hline \multicolumn{3}{|l|}{ Flow and timing } \\
\hline \multicolumn{3}{|l|}{ Comparative } \\
\hline \multicolumn{3}{|l|}{ Notes } \\
\hline \multicolumn{3}{|l|}{ Methodological quality } \\
\hline Item & Authors' judgement & $\begin{array}{l}\text { Applicability con- } \\
\text { cerns }\end{array}$ \\
\hline
\end{tabular}

\section{DOMAIN 1: Patient Selection}

\begin{tabular}{llll}
\hline Was a consecutive or random sample of patients enrolled? & Unclear & \\
\hline Was a case-control design avoided? & Yes & & Low \\
\hline Did the study avoid inappropriate exclusions? & Yes & Unclear \\
\hline
\end{tabular}

\section{DOMAIN 2: Index Test Xpert MTB/RIF}

Were the index test results interpreted without knowledge of Yes the results of the reference standard?

\begin{tabular}{lcl}
\hline If a threshold was used, was it pre-specified? & Yes & \\
\hline Low & Low \\
\hline
\end{tabular}

\section{DOMAIN 3: Reference Standard}

Is the reference standards likely to correctly classify the target Yes condition? 
Shenai 2016 (Continued)

Were the reference standard results for TB detection interpret- Yes ed without knowledge of the results of the index test?

Were the reference standard results for rifampicin resistance detection interpreted without knowledge of the results of the index test?

\section{Low}

Low

\section{DOMAIN 4: Flow and Timing}

Was there an appropriate interval between index test and refer- Yes ence standard?

Did all patients receive the same reference standard? Yes

Were all patients included in the analysis? Yes

Low

\section{Singh 2016}

\section{Study characteristics}

Patient sampling

Cross-sectional design, unknown manner of enrolment, prospective data collection

\section{Patient characteristics and setting}

Presenting signs and symptoms: presumptive pulmonary TB

Age: range 15 to 60 years

Sex, female: not reported

HIV infection: $0 \%$

History of TB: not reported

Sample size: 72

Clinical setting: not reported

Laboratory level: central

Country: India

World Bank Income Classification: middle income

High TB burden country: yes

High MDR-TB burden country: yes

High TB/HIV burden country: yes

Index tests Index: Xpert MTB/RIF

\footnotetext{
Target condition and reference standard(s)
}

Target condition: rifampicin resistance 
Singh 2016 (Continued)

Reference standard for rifampicin resistance: MGIT 960

\section{Flow and timing}

\section{Comparative}

\section{Notes}

\section{Methodological quality}

\begin{tabular}{lll}
\hline Item & $\begin{array}{l}\text { Authors' judge- } \\
\text { ment }\end{array}$ & Risk of bias Applicability \\
concerns
\end{tabular}

\section{DOMAIN 1: Patient Selection}

\begin{tabular}{lcc}
\hline Was a consecutive or random sample of patients enrolled? & Unclear \\
\hline Was a case-control design avoided? & Yes & \\
\hline Did the study avoid inappropriate exclusions? & Yes & Unclear \\
\hline
\end{tabular}

\section{DOMAIN 2: Index Test Xpert MTB/RIF}

Were the index test results interpreted without knowledge of the results of Yes the reference standard?

If a threshold was used, was it pre-specified? Yes

\begin{tabular}{lll}
\hline Unclear & Low \\
\hline
\end{tabular}

\section{DOMAIN 3: Reference Standard}

Is the reference standards likely to correctly classify the target condition? Yes

Were the reference standard results for TB detection interpreted without Yes knowledge of the results of the index test?

Were the reference standard results for rifampicin resistance detection in- Yes terpreted without knowledge of the results of the index test?

Low Low

\section{DOMAIN 4: Flow and Timing}

Was there an appropriate interval between index test and reference stan- Yes dard?

Did all patients receive the same reference standard?

Were all patients included in the analysis?
Yes

Yes

Low 


\section{Sohn 2014}

\section{Study characteristics}

\section{Patient sampling}

Cross-sectional design, consecutive enrolment, prospective data collection

\section{Patient characteristics and setting}

Presenting signs and symptoms: presumptive active pulmonary $\mathrm{TB}$, only $18 \%$ of participants were symptomatic

Age: median 44 years (IQR 31 to 61 ), range 18 to $>50$ years

Sex, female: $44 \%$

HIV infection: $2 \%$

History of TB: $22 \%$

Sample size: 501

Clinical setting: outpatient

Laboratory level: central

Country: Canada

World Bank Income Classification: high income

High TB burden country: no

High MDR-TB burden country: no

High TB/HIV burden country: no

Prevalence of TB cases in the study: $5.0 \%$

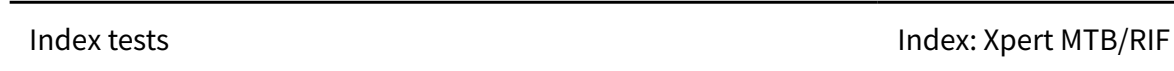

Reference standard for pulmonary TB: MGIT 960

Target condition: rifampicin resistance

Reference standard for rifampicin resistance: MGIT 960

\section{Flow and timing}

\section{Comparative}

\section{Notes}

Only $18 \%$ of the included participants had symptoms suggestive

of active TB (e.g. fever, cough, night sweats, weight loss)

\section{Methodological quality}

\begin{tabular}{lll}
\hline Item Authors' judgement & Risk of bias $\begin{array}{l}\text { Applicability con- } \\
\text { cerns }\end{array}$
\end{tabular}

\section{DOMAIN 1: Patient Selection}

Was a consecutive or random sample of patients enrolled? 
Sohn 2014 (Continued)

Was a case-control design avoided?

Yes

Did the study avoid inappropriate exclusions?

Yes

\begin{tabular}{lcc}
\hline Low & Low \\
\hline
\end{tabular}

\section{DOMAIN 2: Index Test Xpert MTB/RIF}

Were the index test results interpreted without knowledge of Yes the results of the reference standard?

\begin{tabular}{lcc}
\hline If a threshold was used, was it pre-specified? & Yes & \\
\hline Low & Low \\
\hline
\end{tabular}

\section{DOMAIN 3: Reference Standard}

Is the reference standards likely to correctly classify the target Yes condition?

Were the reference standard results for TB detection interpret- Yes ed without knowledge of the results of the index test?

Were the reference standard results for rifampicin resistance detection interpreted without knowledge of the results of the index test?

Low

Low

\section{DOMAIN 4: Flow and Timing}

Was there an appropriate interval between index test and refer- Yes ence standard?

Did all patients receive the same reference standard? Yes

Were all patients included in the analysis? Yes

Low

\section{Ssengooba 2014}

\section{Study characteristics}

Patient sampling

Cross-sectional design, random enrolment, prospective study design

Patient characteristics and setting

Presenting signs and symptoms: clinical TB symptoms

Age: 18 years and older, median 33 years (IQR 29 to 37 )

Sex, female: $63 \%$

HIV infection: $100 \%$

History of TB: not reported 


\section{Sample size: 424}

Clinical setting: inpatient and outpatient

Laboratory level: central

Country: Uganda

World Bank Income Classification: low income

High TB burden country: no

High MDR-TB burden country: no

High TB/HIV burden country: yes

Prevalence of TB cases in the study: $29.0 \%$

\begin{tabular}{ll}
\hline Index tests & Index: Xpert MTB/RIF \\
\hline Target condition and reference standard(s) & Target condition: pulmonary TB \\
& Reference standard for pulmonary TB: LJ and MGIT 960 \\
& Target condition: rifampicin resistance \\
& Reference standard for rifampicin resistance: MGIT 960
\end{tabular}

Flow and timing

Comparative

\begin{tabular}{|c|c|c|c|}
\hline Notes & \multicolumn{3}{|c|}{ Substudy of Nakiyingi 2014} \\
\hline \multicolumn{4}{|c|}{ Methodological quality } \\
\hline Item & $\begin{array}{l}\text { Authors' judge- } \\
\text { ment }\end{array}$ & Risk of bias & $\begin{array}{l}\text { Applicability con- } \\
\text { cerns }\end{array}$ \\
\hline
\end{tabular}

DOMAIN 1: Patient Selection

\begin{tabular}{lcc}
\hline Was a consecutive or random sample of patients enrolled? & Yes & Yes \\
\hline Was a case-control design avoided? & Yes & Low Low \\
\hline Did the study avoid inappropriate exclusions? & & Low \\
\hline
\end{tabular}

\section{DOMAIN 2: Index Test Xpert MTB/RIF}

Were the index test results interpreted without knowledge of the re- Yes sults of the reference standard?

\section{DOMAIN 3: Reference Standard}




\section{Ssengooba 2014 (Continued)}

Is the reference standards likely to correctly classify the target con- Yes dition?

Were the reference standard results for TB detection interpreted Yes without knowledge of the results of the index test?

Were the reference standard results for rifampicin resistance detec- Yes

tion interpreted without knowledge of the results of the index test?

\begin{tabular}{lll}
\hline DOMAIN 4: Flow and Timing & Low & \\
\hline Was there an appropriate interval between index test and reference & Yes \\
standard? & Yes \\
\hline Did all patients receive the same reference standard? & Yes & Low
\end{tabular}

\section{Tadesse 2016}

\section{Study characteristics}

Patient sampling Cross-sectional design, consecutive enrolment of participants, prospective data collection

Patient characteristics and setting

Presenting signs and symptoms: clinical suspicion of TB, smear-negative

Age: 18 years and older, median 38 years (IQR 23 to 55 )

Sex, female: $38 \%$

HIV infection: $0 \%$

History of TB: not reported

Sample size: 185

Clinical setting: not reported

Laboratory level: central

Country: Ethiopia

World Bank Income Classification: low income

High TB burden country: yes

High MDR-TB burden country: yes

High TB/HIV burden country: yes

Prevalence of TB cases in the study: $10.3 \%$ 
Tadesse 2016 (Continued)
Index tests
Index: Xpert MTB/RIF

Target condition and reference standard(s)

Target condition: pulmonary TB

Reference standard for pulmonary TB: LJ and MGIT 960

Target condition: rifampicin resistance

Reference standard for rifampicin resistance: LJ and MGIT 960

\begin{tabular}{ll} 
Flow and timing \\
\hline Comparative \\
\hline Notes \\
$\begin{array}{ll} & \text { "One hundred twenty-four patients were excluded from the study ( } 56 \\
& \text { were HIV-positive/unknown, } 30 \text { were smear positive, } 19 \text { provided a sam- } \\
& \text { ple with inadequate volume, } 13 \text { did not provide three sputa, and six had } \\
& \text { missing acid-fast bacilli-smear results)." }\end{array}$
\end{tabular}

\section{Methodological quality}

\begin{tabular}{llll}
\hline Item Authors' judgement & Risk of bias & $\begin{array}{l}\text { Applicability con- } \\
\text { cerns }\end{array}$
\end{tabular}

\section{DOMAIN 1: Patient Selection} Was a consecutive or random sample of patients en- $\quad$ Yes
rolled?

\begin{tabular}{|c|c|c|}
\hline Was a case-control design avoided? & Yes & \\
\hline \multirow[t]{2}{*}{ Did the study avoid inappropriate exclusions? } & No & \\
\hline & High & Unclear \\
\hline
\end{tabular}

DOMAIN 2: Index Test Xpert MTB/RIF

Were the index test results interpreted without knowledge Yes

of the results of the reference standard?

If a threshold was used, was it pre-specified? Yes

Low
Low L L

\section{DOMAIN 3: Reference Standard}

Is the reference standards likely to correctly classify the Yes

target condition?

Were the reference standard results for TB detection inter- Yes preted without knowledge of the results of the index test?

Were the reference standard results for rifampicin resis- Yes tance detection interpreted without knowledge of the results of the index test? 
Tadesse 2016 (Continued)

DOMAIN 4: Flow and Timing

Was there an appropriate interval between index test and Yes reference standard?

Did all patients receive the same reference standard? Yes

Were all patients included in the analysis? Yes

Low

\section{Tang 2017}

\section{Study characteristics}

$\begin{array}{ll}\text { Patient sampling } & \begin{array}{l}\text { Cross-sectional design, consecutive enrolment, prospective data } \\ \text { collection }\end{array}\end{array}$

Patient characteristics and setting

Presenting signs and symptoms: clinical suspicion of TB

Age: median 36 years, range 16 to 78 years

Sex, female: $47 \%$

HIV infection: not reported

History of TB: not reported

Sample size: 240

Clinical setting: not reported

Laboratory level: central

Country: China

World Bank Income Classification: middle income

High TB burden country: yes

High MDR-TB burden country: yes

High TB/HIV burden country: yes

Prevalence of TB cases in the study: $36.0 \%$

Index tests Index: Xpert MTB/RIF

Target condition and reference standard(s)

Target condition: pulmonary TB

Reference standard for pulmonary TB: MGIT 960

Target condition: rifampicin resistance

Reference standard for rifampicin resistance: MGIT 960

Flow and timing

Comparative

Xpert MTB/RIF and Xpert MTB/RIF Ultra for pulmonary tuberculosis and rifampicin resistance in adults (Review) 
Tang 2017 (Continued)

Notes

Study authors considered the quality of specimens, collection, transport, and testing times as possible explanations for low Xpert

specificity in this study

\section{Methodological quality}

\begin{tabular}{llll}
\hline Item & Authors' judgement & Risk of bias & $\begin{array}{l}\text { Applicability con- } \\
\text { cerns }\end{array}$ \\
\hline
\end{tabular}

\section{DOMAIN 1: Patient Selection}

\begin{tabular}{lcc}
\hline Was a consecutive or random sample of patients enrolled? & Yes \\
\hline Was a case-control design avoided? & Yes & \\
\hline Did the study avoid inappropriate exclusions? & Yes & Low \\
\hline
\end{tabular}

\section{DOMAIN 2: Index Test Xpert MTB/RIF}

Were the index test results interpreted without knowledge of Yes the results of the reference standard?

If a threshold was used, was it pre-specified? Yes

\begin{tabular}{lll}
\hline & Low & Low \\
\hline DOMAIN 3: Reference Standard &
\end{tabular}

\section{DOMAIN 3: Reference Standard}

Is the reference standards likely to correctly classify the target Yes condition?

Were the reference standard results for TB detection interpret- Yes ed without knowledge of the results of the index test?

Were the reference standard results for rifampicin resistance detection interpreted without knowledge of the results of the index test?

\begin{tabular}{lll}
\hline & Low & Low \\
\hline DOMAIN 4: Flow and Timing & & \\
\hline $\begin{array}{l}\text { Was there an appropriate interval between index test and refer- } \\
\text { ence standard? }\end{array}$ & Yes \\
\hline Did all patients receive the same reference standard? & Yes \\
\hline Were all patients included in the analysis? &
\end{tabular}

Low 
Theron 2011

\section{Study characteristics}

Patient sampling

Patient characteristics and setting
Cross-sectional design, consecutive enrolment, retrospective data collection

Presenting signs and symptoms: presumptive TB based on compatible signs and symptoms

Age: median 36 years, range 18 to 83 years

Sex, female: $32.3 \%$

HIV infection: $31.3 \%$

History of TB: $34.3 \%$

Sample size: 480

Clinical setting: 2 primary care clinics in a high HIV prevalence area

Laboratory level: central

Country: South Africa, Cape Town

World Bank Income Classification: middle income

High TB burden country: yes

High MDR-TB burden country: yes

High TB/HIV burden country: yes

TB incidence rate: 993 per 100,000

MDR-TB prevalence: percentage MDR-TB among new TB cases $=0.9 \%$ (Source: survey in Western Cape Province, 2002) and among retreatment cases $=4.0 \%$ (Source: survey in Western Cape Province, 2002)

Prevalence of TB cases in the study: $29.4 \%$

Index tests Index: Xpert MTB/RIF

Target condition and reference standard(s)

Target condition: pulmonary TB

Reference standard for pulmonary TB: MGIT 960

Target condition: rifampicin resistance

Reference standard for rifampicin resistance: MGIT 960

Flow and timing

Comparative

Notes

Short-term follow-up cultures were obtained; 16 of 19 Xpert MTB/RIF-positive culture-negative participants were considered likely to be TB cases based on follow-up cultures, gene sequencing, and the presence of characteristic radiographic features using a standardized scoring system

\section{Methodological quality}

\section{Item}

Authors' judgement

Risk of bias

Applicability concerns

Xpert MTB/RIF and Xpert MTB/RIF Ultra for pulmonary tuberculosis and rifampicin resistance in adults (Review) 
Theron 2011 (Continued)

\section{DOMAIN 1: Patient Selection}

Was a consecutive or random sample of patients Yes enrolled?

\begin{tabular}{lccl}
\hline Was a case-control design avoided? & Yes & \\
\hline Did the study avoid inappropriate exclusions? & Yes & Low & Low \\
\hline
\end{tabular}

DOMAIN 2: Index Test Xpert MTB/RIF

Were the index test results interpreted without dard?

If a threshold was used, was it pre-specified? Yes

\begin{tabular}{lll}
\hline Low & Low \\
\hline DOMAIN 3: Reference Standard & &
\end{tabular}

Is the reference standards likely to correctly clas- Yes sify the target condition?

Were the reference standard results for TB detection interpreted without knowledge of the results of the index test?

Were the reference standard results for rifampicin Yes resistance detection interpreted without knowl-

edge of the results of the index test?

\begin{tabular}{lcc}
\hline & Low & Low \\
\hline DOMAIN 4: Flow and Timing &
\end{tabular}

\section{Was there an appropriate interval between index Yes} test and reference standard?

Did all patients receive the same reference stan- Yes dard?

Were all patients included in the analysis? Yes

\section{Low}

\section{Theron 2013}

\section{Study characteristics}

Patient sampling

Cross-sectional design, consecutive enrolment, prospective data collection 


\section{Theron 2013 (Continued)}

Patient characteristics and setting
Presenting signs and symptoms: presumptive pulmonary TB, sputum scarce or smear-negative

Age: 18 years and older, median 46 years (IQR 33 to 56 )

Sex, female: $46 \%$

HIV infection: $30 \%$

History of TB: $34 \%$

Sample size: 154

Clinical setting: not reported

Laboratory level: central

Country: South Africa

World Bank Income Classification: middle income

High TB burden country: yes

High MDR-TB burden country: yes

High TB/HIV burden country: yes

Prevalence of TB cases in the study: $17.8 \%$

\begin{tabular}{ll}
\hline Index tests & Index: Xpert MTB/RIF \\
\hline Target condition and reference standard(s) & Target condition: pulmonary TB \\
& $\begin{array}{l}\text { Reference standard for pulmonary TB: MGIT 960 } \\
\text { Target condition: rifampicin resistance }\end{array}$ \\
\hline Flow and timing & Reference standard for rifampicin resistance: MGIT 960 \\
\hline Comparative & \\
\hline Notes & $\begin{array}{l}\text { Risk of bias } \\
\text { Methodological quality }\end{array}$ \\
\hline Item & $\begin{array}{l}\text { Applicability con- } \\
\text { cerns }\end{array}$
\end{tabular}

\section{DOMAIN 1: Patient Selection}

\begin{tabular}{lcc}
\hline Was a consecutive or random sample of patients enrolled? & Yes \\
\hline Was a case-control design avoided? & Yes & No \\
\hline Did the study avoid inappropriate exclusions? & High
\end{tabular}

\section{DOMAIN 2: Index Test Xpert MTB/RIF}


Theron 2013 (Continued)

Were the index test results interpreted without knowledge of the re- Yes sults of the reference standard?

\begin{tabular}{lcc}
\hline If a threshold was used, was it pre-specified? & Yes \\
\hline Low & Low \\
\hline
\end{tabular}

\section{DOMAIN 3: Reference Standard}

Is the reference standards likely to correctly classify the target con- Yes dition?

Were the reference standard results for TB detection interpreted Yes without knowledge of the results of the index test?

Were the reference standard results for rifampicin resistance detec- Yes tion interpreted without knowledge of the results of the index test?

\begin{tabular}{lll}
\hline & Low & Low \\
\hline DOMAIN 4: Flow and Timing & Yes & \\
\hline $\begin{array}{l}\text { Was there an appropriate interval between index test and reference } \\
\text { standard? }\end{array}$ & Yes Yes & Low \\
\hline Did all patients receive the same reference standard? & \\
\hline Were all patients included in the analysis?
\end{tabular}

\section{Theron 2014a}

\section{Study characteristics}

Patient sampling

Randomized, parallel-group, multicentre trial, prospective data collection

\section{Patient characteristics and setting}

Presenting signs and symptoms: 1 or more symptoms of pulmonary TB according to predefined WHO criteria

Age: 18 years or older, median 37 years (IQR 30 to 46)

Sex, female: $43 \%$

HIV infection: $69 \%$

History of TB: not reported

Sample size: 729

Clinical setting: outpatient

Laboratory level: peripheral

Country: South Africa, Zimbabwe, Zambia, and Tanzania

World Bank Income Classification: low and middle income 
Theron 2014a (Continued)

High TB burden country: yes (South Africa), yes (Zimbabwe), yes (Zambia), yes (Tanzania)

High MDR-TB burden country: yes (South Africa), yes (Zimbabwe), no (Zambia), no (Tanzania)

High TB/HIV burden country: yes (South Africa), yes (Zimbabwe), yes (Zambia), yes (Tanzania)

Prevalence of TB cases in the study: $25.4 \%$

Index tests

Target condition and reference standard(s)

\section{Index: Xpert MTB/RIF}

Target condition: pulmonary TB

Reference standard for pulmonary TB: MGIT 960

Flow and timing

Comparative

Notes

\section{Methodological quality}

\begin{tabular}{llll}
\hline Item Authors' judgement & Risk of bias & $\begin{array}{l}\text { Applicability con- } \\
\text { cerns }\end{array}$
\end{tabular}

\section{DOMAIN 1: Patient Selection}

\begin{tabular}{llll}
\hline Was a consecutive or random sample of patients enrolled? & Yes \\
\hline Was a case-control design avoided? & Yes & \\
\hline Did the study avoid inappropriate exclusions? & Yes & Low & Low \\
\hline
\end{tabular}

\section{DOMAIN 2: Index Test Xpert MTB/RIF}

Were the index test results interpreted without knowledge of Yes the results of the reference standard?

\begin{tabular}{lll}
\hline If a threshold was used, was it pre-specified? & Yes & \\
\hline Low & Low \\
\hline
\end{tabular}

\section{DOMAIN 3: Reference Standard}

Is the reference standards likely to correctly classify the target Yes condition?

Were the reference standard results for TB detection interpreted without knowledge of the results of the index test?

Were the reference standard results for rifampicin resistance detection interpreted without knowledge of the results of the index test? 
Theron 2014a (Continued)

\section{DOMAIN 4: Flow and Timing}

Was there an appropriate interval between index test and ref- Yes erence standard?

\begin{tabular}{ll}
\hline Did all patients receive the same reference standard? Yes & Y
\end{tabular}

Were all patients included in the analysis? Yes

Low

\section{Tsuyuguchi 2017}

\section{Study characteristics}

\section{Patient sampling}

Cross-sectional design, consecutive enrolment, prospective data collection

\section{Patient characteristics and setting}

Presenting signs and symptoms: presumed TB

Age: mean 65 years (SD 17), range 23 to 94 years

Sex, female: $38 \%$

HIV infection: not reported

History of TB: not reported

Sample size: 417

Clinical setting: not reported

Laboratory level: central

Country: Japan

World Bank Income Classification: high income

High TB burden country: no

High MDR-TB burden country: no

High TB/HIV burden country: no

Prevalence of TB cases in the study: $55.0 \%$

\begin{tabular}{ll}
\hline Index tests & Index: Xpert MTB/RIF \\
\hline Target condition and reference standard(s) & Target condition: pulmonary TB \\
& Reference standard for pulmonary TB: Ogawa and MGIT 960 \\
& Target condition: rifampicin resistance \\
& Reference standard for rifampicin resistance: MGIT 960
\end{tabular}


Tsuyuguchi 2017 (Continued)

Flow and timing

Comparative
A total of 515 sputum specimens were collected; however, 35 were ineligible due to over-testing

\section{Notes}

\section{Methodological quality}

\begin{tabular}{llll}
\hline Item & $\begin{array}{l}\text { Authors' judge- } \\
\text { ment }\end{array}$ & Risk of bias & $\begin{array}{l}\text { Applicability con- } \\
\text { cerns }\end{array}$
\end{tabular}

\section{DOMAIN 1: Patient Selection}

\begin{tabular}{lc}
\hline Was a consecutive or random sample of patients enrolled? & Yes \\
\hline Was a case-control design avoided? & Yes \\
\hline Did the study avoid inappropriate exclusions? & Yes
\end{tabular}

\begin{tabular}{lll}
\hline & Low & Unclear \\
\hline DOMAIN 2: Index Test Xpert MTB/RIF & &
\end{tabular}

\section{DOMAIN 2: Index Test Xpert MTB/RIF}

Were the index test results interpreted without knowledge of the Yes results of the reference standard?

\begin{tabular}{lcc}
\hline If a threshold was used, was it pre-specified? & Yes & Low Low \\
\hline
\end{tabular}

\section{DOMAIN 3: Reference Standard}

Is the reference standards likely to correctly classify the target Yes condition?

Were the reference standard results for TB detection interpreted Yes without knowledge of the results of the index test?

Were the reference standard results for rifampicin resistance de- Yes tection interpreted without knowledge of the results of the index test?

\begin{tabular}{lll}
\hline & Low & Low \\
\hline DOMAIN 4: Flow and Timing & Yes & \\
\hline $\begin{array}{l}\text { Was there an appropriate interval between index test and refer- } \\
\text { ence standard? }\end{array}$ & Yes \\
\hline Did all patients receive the same reference standard? & & Unclear \\
\hline Were all patients included in the analysis? & \\
\hline
\end{tabular}


Van Rie 2013

\section{Study characteristics}

Patient sampling Cross-sectional design, consecutive enrolment, prospective data collection

Patient characteristics and setting

Presenting signs and symptoms: prolonged (> 2 weeks) cough or other TB symptoms, or both, and had 2 prior-negative smear by fluorescence microscopy

Age: median 36 years (IQR 30 to 34 )

Sex, female: $56.8 \%$

HIV infection: $72.4 \%$

History of TB: $17.6 \%$

Sample size: 161

Clinical setting: primary care clinic

Laboratory level: peripheral

Country: South Africa, Johannesburg

World Bank Income Classification: middle income

High TB burden country: yes

High MDR-TB burden country: yes

High TB/HIV burden country: yes

TB incidence rate: 993 per 100,000

MDR-TB prevalence: percentage MDR-TB among new TB cases $=1.4 \%$

(Source: survey in Gauteng province, 2002) and among retreatment cases $=$ $5.5 \%$ (Source: survey in Gauteng province, 2002)

Prevalence of TB cases in the study: 9.3\%

Index tests Index: Xpert MTB/RIF

Target condition and reference standard(s)

Target condition: pulmonary TB

Reference standard for pulmonary TB: MGIT 960

Target condition: rifampicin resistance

Reference standard for rifampicin resistance: MGIT 960

\section{Flow and timing}

Only those participants presumed to have TB who returned for results of the initial smear microscopy examinations were enrolled

\section{Comparative}

Notes

\section{Methodological quality}


Van Rie 2013 (Continued)

Item

Authors' judgement

Risk of bias

Applicability con-

cerns

\section{DOMAIN 1: Patient Selection}

Was a consecutive or random sample of patients en- Yes rolled?

\begin{tabular}{llll}
\hline Was a case-control design avoided? & Yes & \\
\hline Did the study avoid inappropriate exclusions? & No & High & Low \\
\hline
\end{tabular}

\section{DOMAIN 2: Index Test Xpert MTB/RIF}

Were the index test results interpreted without knowl- Yes edge of the results of the reference standard?

\begin{tabular}{lll}
\hline If a threshold was used, was it pre-specified? & Yes & Low \\
\hline
\end{tabular}

\section{DOMAIN 3: Reference Standard}

Is the reference standards likely to correctly classify Yes the target condition?

Were the reference standard results for TB detection Yes interpreted without knowledge of the results of the index test?

Were the reference standard results for rifampicin resistance detection interpreted without knowledge of the results of the index test?

\begin{tabular}{lll}
\hline DOMAIN 4: Flow and Timing & Low & Low \\
\hline $\begin{array}{l}\text { Was there an appropriate interval between index test } \\
\text { and reference standard? }\end{array}$ & Yes \\
\hline Did all patients receive the same reference standard? & Yes \\
\hline Were all patients included in the analysis? & No \\
\hline
\end{tabular}


Walusimbi 2013a (Continued)
Patient sampling
Cross-sectional design, unknown manner of enrolment, prospective data collection

Patient characteristics and setting

Presenting signs and symptoms: cough for $>2$ weeks, with or without fever, night sweats, loss of weight, or blood-stained sputum, smear-negative

Age: adults, median 34 years (IQR 29 to 40)

Sex, female: $56 \%$

HIV infection: $100 \%$

History of TB: not reported

Sample size: 601

Clinical setting: inpatient and outpatient

Laboratory level: central

Country: Uganda

World Bank Income Classification: low income

High TB burden country: no

High MDR-TB burden country: no

High TB/HIV burden country: yes

Prevalence of TB cases in the study: $11.7 \%$

\begin{tabular}{ll}
\hline Index tests & Index: Xpert MTB/RIF \\
\hline Target condition and reference standard(s) & Target condition: pulmonary TB \\
& Reference standard for pulmonary TB: LJ and MGIT 960
\end{tabular}

Flow and timing

\section{Comparative}

Notes

\section{Methodological quality}

\begin{tabular}{|c|c|c|c|}
\hline Item & $\begin{array}{l}\text { Authors' judge- } \\
\text { ment }\end{array}$ & Risk of bias & $\begin{array}{l}\text { Applicability } \\
\text { concerns }\end{array}$ \\
\hline \multicolumn{4}{|l|}{ DOMAIN 1: Patient Selection } \\
\hline Was a consecutive or random sample of patients enrolled? & Unclear & & \\
\hline Was a case-control design avoided? & Yes & & \\
\hline \multirow[t]{2}{*}{ Did the study avoid inappropriate exclusions? } & No & & \\
\hline & & High & Low \\
\hline
\end{tabular}

\section{DOMAIN 2: Index Test Xpert MTB/RIF}

Xpert MTB/RIF and Xpert MTB/RIF Ultra for pulmonary tuberculosis and rifampicin resistance in adults (Review) 
Walusimbi 2013a (Continued)

Were the index test results interpreted without knowledge of the re- Yes sults of the reference standard?

If a threshold was used, was it pre-specified?

Yes

\begin{tabular}{lll}
\hline Low & Low \\
\hline
\end{tabular}

\section{DOMAIN 3: Reference Standard}

Is the reference standards likely to correctly classify the target condi- Yes tion?

Were the reference standard results for TB detection interpreted without knowledge of the results of the index test?

Were the reference standard results for rifampicin resistance detec-

tion interpreted without knowledge of the results of the index test?

\begin{tabular}{lll}
\hline & Low & Low \\
\hline DOMAIN 4: Flow and Timing & Yes \\
\hline $\begin{array}{l}\text { Was there an appropriate interval between index test and reference } \\
\text { standard? }\end{array}$ & Yes \\
\hline Did all patients receive the same reference standard? & Yes \\
\hline Were all patients included in the analysis? &
\end{tabular}

Low

Williamson 2012

\section{Study characteristics}

Patient sampling

Patient characteristics and setting
Cross-sectional design, consecutive enrolment, prospective data collection

Presenting signs and symptoms: not reported: smear-positive specimens

Age: 15 years and older

Sex, female: not reported

HIV infection: estimated $<1 \%$

History of TB: not reported

Sample size: 89

Clinical setting: laboratory-based

Laboratory level: central

Country: New Zealand

World Bank Income Classification: high income 
Williamson 2012 (Continued)

High TB burden country: no

High MDR-TB burden country: no

High TB/HIV burden country: no

TB incidence rate: 7.6 per 100,000

MDR-TB prevalence: percentage MDR-TB among new $\mathrm{TB}$ cases = $2.5 \%$ (Source: nationwide surveillance 2009 ) and among retreatment cases $=13 \%$ (Source: nationwide surveillance 2009)

Prevalence of TB cases in the study: $75.3 \%$

\begin{tabular}{ll}
\hline Index tests & Index: Xpert MTB/RIF \\
\hline Target condition and reference standard(s) & Target condition: pulmonary TB \\
& Reference standard for pulmonary TB: MGIT 960 \\
& Target condition: rifampicin resistance \\
& Reference standard for rifampicin resistance: MGIT 960 \\
\hline
\end{tabular}

Flow and timing

Comparative

Notes

Methodological quality

\begin{tabular}{llll}
\hline Item Authors' judgement & Risk of bias $\begin{array}{l}\text { Applicability con- } \\
\text { cerns }\end{array}$ \\
\hline
\end{tabular}

\section{DOMAIN 1: Patient Selection}

\begin{tabular}{lll}
\hline Was a consecutive or random sample of patients enrolled? & Yes \\
\hline Was a case-control design avoided? & Yes \\
\hline Did the study avoid inappropriate exclusions? & No & High \\
\hline
\end{tabular}

DOMAIN 2: Index Test Xpert MTB/RIF

Were the index test results interpreted without knowledge of Yes the results of the reference standard?

If a threshold was used, was it pre-specified? Yes

\begin{tabular}{llll}
\hline Low & Low \\
\hline
\end{tabular}

\section{DOMAIN 3: Reference Standard}

Is the reference standards likely to correctly classify the target Yes condition? 
Williamson 2012 (Continued)

Were the reference standard results for TB detection inter- Yes preted without knowledge of the results of the index test?

Were the reference standard results for rifampicin resistance

Yes detection interpreted without knowledge of the results of the index test?

Low

Low

\section{DOMAIN 4: Flow and Timing}

Was there an appropriate interval between index test and ref- Yes erence standard?

\begin{tabular}{|ll}
\hline Did all patients receive the same reference standard? Yes & Y
\end{tabular}

Were all patients included in the analysis? Yes

Yoon 2017

\section{Study characteristics}

Patient sampling

\section{Patient characteristics and setting}

Cross-sectional design, consecutive enrolment, prospective data collection

Presenting signs and symptoms: HIV-positive people initiating antiretroviral therapy

Age: 18 years and older, median 33 years (IQR 27 to 40 )

Sex, female: $53 \%$

HIV infection: $100 \%$

History of TB: $4 \%$

Sample size: 1177

Clinical setting: outpatient HIV/AIDS clinics

Laboratory level: central

Country: Uganda

World Bank Income Classification: middle income

High TB burden country: no

High MDR-TB burden country: no

High TB/HIV burden country: yes

Prevalence of TB cases in the study: $13.8 \%$

\section{Index tests}

Index: Xpert MTB/RIF

\footnotetext{
Target condition and reference standard(s)
}

Target condition: pulmonary TB 
Yoon 2017 (Continued)

Flow and timing

Comparative

Notes

\section{Methodological quality}

\begin{tabular}{|c|c|c|c|}
\hline Item & $\begin{array}{l}\text { Authors' judge- } \\
\text { ment }\end{array}$ & Risk of bias & $\begin{array}{l}\text { Applicability } \\
\text { concerns }\end{array}$ \\
\hline
\end{tabular}

\section{DOMAIN 1: Patient Selection}

\begin{tabular}{lc}
\hline Was a consecutive or random sample of patients enrolled? & Yes \\
\hline Was a case-control design avoided? & Yes \\
\hline Did the study avoid inappropriate exclusions? & Yes \\
\hline
\end{tabular}

\begin{tabular}{lll}
\hline & Low & Low \\
\hline DOMAIN 2: Index Test Xpert MTB/RIF &
\end{tabular}

Were the index test results interpreted without knowledge of the results Yes of the reference standard?

\begin{tabular}{lll}
\hline If a threshold was used, was it pre-specified? & Yes & Low Low \\
\hline
\end{tabular}

\section{DOMAIN 3: Reference Standard}

Is the reference standards likely to correctly classify the target condi- Yes tion?

Were the reference standard results for TB detection interpreted with- Yes out knowledge of the results of the index test?

Were the reference standard results for rifampicin resistance detection interpreted without knowledge of the results of the index test?

\begin{tabular}{lll}
\hline DOMAIN 4: Flow and Timing & Low & Low \\
\hline $\begin{array}{l}\text { Was there an appropriate interval between index test and reference } \\
\text { standard? }\end{array}$ & Yes & Yes \\
\hline Did all patients receive the same reference standard? & Yes \\
\hline Were all patients included in the analysis?
\end{tabular}


Zeka 2011

\section{Study characteristics}

Patient sampling

Cross-sectional design, consecutive enrolment, prospective data collection

\section{Patient characteristics and setting}

Presenting signs and symptoms: clinical findings of possible TB

Age: median 48 years, range 25 to 70 years

Sex, female: $42.4 \%$

HIV infection: not reported

History of TB: not reported

Sample size: 103

Clinical setting: laboratory-based

Laboratory level: central

Country: Turkey

World Bank Income Classification: middle income

High TB burden country: no

High MDR-TB burden country: no

High TB/HIV burden country: no

TB incidence rate: 24 per 100,000

MDR-TB prevalence: percentage MDR-TB among new TB cases $=0.9 \%$

(Source: survey in Ankara City 2011) and among retreatment cases $=38 \%$

(Source: survey in Ankara City 2011)

Prevalence of TB cases in the study: $34.0 \%$

Index tests

Target condition and reference standard(s)
Index: Xpert MTB/RIF

Target condition: pulmonary TB

Reference standard for pulmonary TB: LJ culture and MB/MBacT liquid medium

Target condition: rifampicin resistance

Reference standard for rifampicin resistance: proportion method on $7 \mathrm{H} 10$ media

\section{Flow and timing}

\section{Comparative}

\section{Notes}

Only one rifampicin resistant isolate was identified. Data for sputum specimens were provided by the study author

\section{Methodological quality}


Zeka 2011 (Continued)

Item

Authors' judgement Risk of bias

Applicability con-

cerns

DOMAIN 1: Patient Selection

Was a consecutive or random sample of patients en- Yes rolled?

\begin{tabular}{|c|c|c|}
\hline Was a case-control design avoided? & Yes & \\
\hline Did the study avoid inappropriate exclusions? & Yes & \\
\hline
\end{tabular}

\section{DOMAIN 2: Index Test Xpert MTB/RIF}

Were the index test results interpreted without knowl- Yes edge of the results of the reference standard?

\begin{tabular}{llll}
\hline If a threshold was used, was it pre-specified? & Yes & Low \\
\hline
\end{tabular}

\section{DOMAIN 3: Reference Standard}

Is the reference standards likely to correctly classify the Yes target condition?

Were the reference standard results for TB detection interpreted without knowledge of the results of the index test?

Were the reference standard results for rifampicin resistance detection interpreted without knowledge of the results of the index test?

\begin{tabular}{llll}
\hline & High & Low \\
\hline DOMAIN 4: Flow and Timing & Yes \\
\hline $\begin{array}{l}\text { Was there an appropriate interval between index test } \\
\text { and reference standard? }\end{array}$ & Yes \\
\hline Did all patients receive the same reference standard? & Yes \\
\hline Were all patients included in the analysis? &
\end{tabular}


Zetola 2014 (Continued)

Patient sampling

Patient characteristics and setting

Cross-sectional design, consecutive enrolment, retrospective data collection

Presenting signs and symptoms: (i) people with presumed pulmonary TB at high risk for MDR-TB, (ii) people who had been treated with anti-TB drugs and in whom TB had again been diagnosed, i.e. all retreatment categories (failure, default, and relapse), (iii) HIV-positive people with signs or symptoms of TB, (iv) people who were seriously ill and suspected of having TB regardless of HIV status, and ( $v$ ) people with unknown HIV status presenting with clinical evidence of HIV infection and signs or symptoms of PTB

Age: 18 years or older, median 37 years (IQR 31 to 44)

Sex, female: $40 \%$

HIV infection: $75 \%$

History of TB: $62 \%$

Sample size: 370

Clinical setting: not reported

Laboratory level: central

Country: Botswana

World Bank Income Classification: middle income

High TB burden country: no

High MDR-TB burden country: no

High TB/HIV burden country: yes

\begin{tabular}{ll}
\hline Index tests & Index: Xpert MTB/RIF \\
\hline Target condition and reference standard(s) & Target condition: rifampicin resistance \\
& Reference standard for rifampicin resistance: LJ
\end{tabular}

Flow and timing

Comparative

Notes

\section{Methodological quality}

\begin{tabular}{llll}
\hline Item & Authors' judgement & Risk of bias & $\begin{array}{l}\text { Applicability con- } \\
\text { cerns }\end{array}$ \\
\hline
\end{tabular}

\section{DOMAIN 1: Patient Selection}

Was a consecutive or random sample of patients enrolled?

Yes

\begin{tabular}{ll}
\hline Was a case-control design avoided? & Yes \\
\hline Did the study avoid inappropriate exclusions? & Yes \\
\hline
\end{tabular}

Xpert MTB/RIF and Xpert MTB/RIF Ultra for pulmonary tuberculosis and rifampicin resistance in adults (Review) 
Zetola 2014 (Continued)

Low

Unclear

\section{DOMAIN 2: Index Test Xpert MTB/RIF}

Were the index test results interpreted without knowledge Yes

of the results of the reference standard?

\begin{tabular}{lcl}
\hline If a threshold was used, was it pre-specified? & Yes & \\
\hline & Low & Low \\
\hline
\end{tabular}

\section{DOMAIN 3: Reference Standard}

Is the reference standards likely to correctly classify the Yes target condition?

Were the reference standard results for TB detection interpreted without knowledge of the results of the index test?

Were the reference standard results for rifampicin resis- $\quad$ Yes tance detection interpreted without knowledge of the results of the index test?

Low

Low

\section{DOMAIN 4: Flow and Timing}

Was there an appropriate interval between index test and Yes reference standard?

Did all patients receive the same reference standard? Yes

Were all patients included in the analysis? Yes

Low

\section{Zmak 2013}

\section{Study characteristics}

Patient sampling

Cross-sectional design, consecutive enrolment, prospective data collection

Patient characteristics and setting

Presenting signs and symptoms: presumed pulmonary TB

Age: adults

Sex, female: not reported

HIV infection: not reported

History of TB: not reported

Sample size: 120

Clinical setting: laboratory-based 
Zmak 2013 (Continued)

Laboratory level: central

Country: Croatia

World Bank Income Classification: middle income

High TB burden country: no

High MDR-TB burden country: no

High TB/HIV burden country: no

Prevalence of TB cases in the study: $6.0 \%$

\begin{tabular}{ll}
\hline Index tests & Index: Xpert MTB/RIF \\
\hline Target condition and reference standard(s) & Target condition: pulmonary TB \\
& Reference standard for pulmonary TB: LJ and MGIT 960 \\
& Target condition: rifampicin resistance \\
& Reference standard for rifampicin resistance: LJ
\end{tabular}

Flow and timing

Comparative

Notes

Methodological quality

\begin{tabular}{lll}
\hline Item & $\begin{array}{l}\text { Authors' judge- } \\
\text { ment }\end{array}$ & $\begin{array}{l}\text { Risk of bias } \\
\text { Applicability } \\
\text { concerns }\end{array}$ \\
\hline
\end{tabular}

DOMAIN 1: Patient Selection

\begin{tabular}{lcc}
\hline Was a consecutive or random sample of patients enrolled? & Yes & Yes \\
\hline Was a case-control design avoided? & Yes & Low \\
\hline Did the study avoid inappropriate exclusions? & Unclear \\
\hline
\end{tabular}

\section{DOMAIN 2: Index Test Xpert MTB/RIF}

Were the index test results interpreted without knowledge of the re- Yes

sults of the reference standard?

If a threshold was used, was it pre-specified?

Yes

Low

Low

\section{DOMAIN 3: Reference Standard}

Is the reference standards likely to correctly classify the target condi- Yes tion? 
Zmak 2013 (Continued)

Were the reference standard results for TB detection interpreted with- No out knowledge of the results of the index test?

Were the reference standard results for rifampicin resistance detection interpreted without knowledge of the results of the index test?

No

\begin{tabular}{lll}
\hline DOMAIN 4: Flow and Timing & High & Low \\
\hline Was there an appropriate interval between index test and reference & Yes \\
standard? & Yes \\
\hline Did all patients receive the same reference standard? & Yes & \\
\hline Were all patients included in the analysis? &
\end{tabular}

Low

Abbreviations: HIV: human immunodeficiency virus; ICU: intensive care unit; IQR: interquartile range; LJ: Löwenstein-Jensen; MDR-TB: multidrug-resistant TB; MGIT: mycobacterial growth indicator tube; MODS: microscopic observation drug susceptibility; SD: standard deviation; TB: tuberculosis

\section{Characteristics of excluded studies [ordered by study ID]}

\begin{tabular}{|c|c|}
\hline Study & Reason for exclusion \\
\hline Acuna-Villaorduna 2017 & Duplicate data with additional analyses; Boum 2016 includes same data set \\
\hline Ade 2016 & Includes both adults and children or no information about age of enrolment \\
\hline Adelman 2014 & Abstract \\
\hline Agizew 2017 & Data insufficient for $2 \times 2$ table \\
\hline Agrawal 2016 & Includes both adults and children or no information about age of enrolment \\
\hline Al-Ateah 2012 & Includes both adults and children or no information about age of enrolment \\
\hline Al-Darraji 2016 & Data insufficient for $2 \times 2$ table \\
\hline Alame-Emane 2017 & Data insufficient for $2 \times 2$ table \\
\hline Albay 2016 & Includes both adults and children or no information about age of enrolment \\
\hline Ali 2016 & Abstract \\
\hline Alland 2015 & Abstract \\
\hline Alnimr 2014 & Data insufficient for $2 \times 2$ table \\
\hline Alvarez 2015 & Includes both adults and children or no information about age \\
\hline Alvarez-Uria 2012 & Reference standard not satisfied \\
\hline
\end{tabular}




\begin{tabular}{|c|c|}
\hline Study & Reason for exclusion \\
\hline Alvis-Zakzuk 2017 & Systematic review \\
\hline Andriani 2016 & Abstract \\
\hline Antonenka 2013 & Case-control study \\
\hline Armand 2011 & $\begin{array}{l}\text { This was a case-control study that compared Xpert MTB/RIF with an in-house IS6110-based re- } \\
\text { al-time PCR using TaqMan probes (IS6110-TaqMan assay) for TB detection }\end{array}$ \\
\hline Asencio 2013 & Cost-effectiveness study \\
\hline Aston 2016 & Abstract \\
\hline Atashi 2017 & Data insufficient for $2 \times 2$ table \\
\hline Atehortua 2015 & Includes both adults and children or no information about age of enrolment \\
\hline Atuhumuza 2016 & Abstract \\
\hline Atwine 2015 & Data insufficient for $2 \times 2$ table \\
\hline Auld 2016b & Includes both adults and children \\
\hline Aurin 2014 & Includes both adults and children or no information about age of enrolment \\
\hline Avashia 2016 & Reference standard not satisfied \\
\hline Ayala 2016 & Data insufficient for $2 \times 2$ table \\
\hline Bablishvili 2015 & Includes both adults and children or no information about age of enrolment \\
\hline Badal-Faesen 2017 & Duplicate data with additional analyses; Luetkemeyer 2016 includes same data set \\
\hline Bajrami 2016 & Includes data for pulmonary and extrapulmonary TB combined \\
\hline Balcha 2014a & Xpert was not the index test \\
\hline Banu 2014 & Data insufficient for $2 \times 2$ table \\
\hline Barkham 2016 & Abstract \\
\hline Barnard 2012 & Includes both adults and children or no information about age of enrolment \\
\hline Bates 2013b & This study evaluated Xpert MTB/RIF for the diagnosis of TB in children \\
\hline Biadglegne 2014 & Includes both adults and children or no information about age of enrolment \\
\hline Bilgin 2016 & Includes both adults and children or no information about age of enrolment \\
\hline Bisognin 2018 & Not a diagnostic accuracy study \\
\hline Bjerrum 2015 & Xpert was not the index test \\
\hline Boakye-Appiah 2016 & Data insufficient for $2 \times 2$ table \\
\hline
\end{tabular}




\begin{tabular}{|c|c|}
\hline Study & Reason for exclusion \\
\hline Bojang 2016 & Xpert was not the index test \\
\hline Bonnet 2017 & Data insufficient for $2 \times 2$ table \\
\hline Bowles 2011 & Includes both adults and children or no information about age of enrolment \\
\hline Bunsow 2014a & Includes respiratory specimens and gastric aspirates \\
\hline Capocci 2016 & Abstract \\
\hline Causse 2011 & This study evaluated Xpert MTB/RIF for the diagnosis of extrapulmonary TB \\
\hline Cavanaugh 2016 & Data insufficient for $2 \times 2$ table \\
\hline Cayci 2017 & Includes both adults and children or no information about age of enrolment \\
\hline Celik 2015 & Includes both adults and children or no information about age of enrolment \\
\hline Chakravorty 2017 & Includes both adults and children or no information about age of enrolment \\
\hline Chishty 2016 & Abstract \\
\hline Ciftçi 2011 & Includes both adults and children or no information about age of enrolment \\
\hline Clouse 2012 & Study on patient impact \\
\hline Cross 2014 & Reference standard not satisfied \\
\hline Cross 2015 & Includes both adults and children or no information about age of enrolment \\
\hline Dagnra 2015 & Data insufficient for $2 \times 2$ table \\
\hline Daum 2015 & Xpert not the index test \\
\hline Deggim 2013 & Includes both adults and children or no information about age of enrolment \\
\hline Dierberg 2016 & Data insufficient for $2 \times 2$ table \\
\hline Dorjee 2012 & Case report \\
\hline Dorman 2012 & Prevalence survey \\
\hline Dowdy 2011 & Cost-effectiveness study \\
\hline Feasey 2013 & Data insufficient for $2 \times 2$ table \\
\hline Fernandez 2017 & Abstract \\
\hline FIND 2011 & $\begin{array}{l}\text { This study compared Xpert MTB/RIF G3 and G4. We excluded it because of concern about duplicate } \\
\text { data. In addition, the criteria for the reference standard for rifampicin resistance detection were } \\
\text { not satisfied }\end{array}$ \\
\hline Fong 2017 & Abstract \\
\hline Friedrich 2011a & This study evaluated Xpert MTB/RIF for the diagnosis of pleural TB \\
\hline
\end{tabular}




\begin{tabular}{|c|c|}
\hline Study & Reason for exclusion \\
\hline Gama de Andrade 2017 & Abstract \\
\hline Gelalcha 2017 & Includes both adults and children or no information about age of enrolment \\
\hline Gounder 2014 & Includes both adults and children or no information about age of enrolment \\
\hline Griesel 2016 & Abstract \\
\hline Griesel 2017 & Includes data for pulmonary and extrapulmonary TB combined \\
\hline Guenaoui 2016 & Includes both adults and children or no information about age of enrolment \\
\hline Gupta 2014 & Abstract \\
\hline Gurbanova 2016 & Abstract \\
\hline Gurbanova 2017 & Includes data for pulmonary and extrapulmonary TB combined \\
\hline Gursoy 2016 & Includes both adults and children or no information about age of enrolment \\
\hline Habeenzu 2017 & Includes both adults and children or no information about age of enrolment \\
\hline Hanifa 2016 & Reference standard not satisfied \\
\hline Heidebrecht 2016 & Data insufficient for $2 \times 2$ table \\
\hline Hillemann 2011 & This study evaluated Xpert MTB/RIF for the diagnosis of extrapulmonary TB \\
\hline Hiza 2017 & Not a diagnostic accuracy study \\
\hline Ho 2016 & Community-based screening \\
\hline Horo 2017 & Includes both adults and children or no information about age of enrolment \\
\hline Hu 2014 & Includes both adults and children or no information about age of enrolment \\
\hline Huang 2018 & Includes both adults and children or no information about age of enrolment \\
\hline Huerga 2017 & Xpert was not the index test \\
\hline Ioannidis 2010 & We could not obtain this article \\
\hline Ioannidis 2011 & Includes both adults and children or no information about age of enrolment \\
\hline Iram 2015 & Includes both adults and children or no information about age of enrolment \\
\hline Jafari 2013 & Data insufficient for $2 \times 2$ table \\
\hline Jing 2017 & Includes both adults and children or no information about age of enrolment \\
\hline Jipa 2016 & Abstract \\
\hline Jones-Lopez 2014 & Xpert was not the index test \\
\hline Kang 2016 & Abstract \\
\hline
\end{tabular}




\begin{tabular}{|c|c|}
\hline Study & Reason for exclusion \\
\hline Kaur 2016 & Systematic review \\
\hline Kayigire 2013 & Not a diagnostic accuracy study \\
\hline Kelly-Cirino 2017 & Xpert was not the index test \\
\hline Kerkhoff 2013 & Data insufficient for $2 \times 2$ table \\
\hline Kerkhoff 2014 & Data insufficient for $2 \times 2$ table \\
\hline Khalil 2015 & includes both adults and children or no information about age of enrolment \\
\hline Khan 2016 & Data insufficient for $2 \times 2$ table \\
\hline Kim 2012 & Case-control study \\
\hline Kim CH 2014 & Duplicate data; Kim CH 2015 includes the same data with more participants \\
\hline Kim MJ 2015 & Data insufficient for $2 \times 2$ table \\
\hline Kim YW 2015 & Includes both adults and children or no information about age of enrolment \\
\hline Lange 2017 & Systematic review \\
\hline Laskar 2017 & Could not obtain full text \\
\hline Lawn 2012a & Study on patient impact \\
\hline Lawn 2012b & Data insufficient for $2 \times 2$ table \\
\hline Lawn 2012c & Primarily a lipoarabinomannan detection study \\
\hline Lawn 2013 & Data insufficient for $2 \times 2$ table \\
\hline Lawn 2015 & Reference standard not satisfied \\
\hline Lawn 2017 & Reference standard not satisfied \\
\hline Lebina 2016 & Community-based screening \\
\hline Lessells 2017 & Impact study \\
\hline Li 2016 & Includes both adults and children or no information about age of enrolment \\
\hline Li 2017 & Systematic review \\
\hline Ligthelm 2011 & This study evaluated Xpert MTB/RIF for the diagnosis of TB lymphadenitis \\
\hline Lombardi 2017 & Includes both adults and children or no information about age of enrolment \\
\hline Mafort 2017 & Abstract \\
\hline Malbruny 2011 & Includes both adults and children or no information about age of enrolment \\
\hline Marlowe 2011 & Includes both adults and children or no information about age of enrolment \\
\hline
\end{tabular}




\begin{tabular}{|c|c|}
\hline Study & Reason for exclusion \\
\hline Matabane 2015 & Includes both adults and children or no information about age of enrolment \\
\hline Mave 2017 & Screening \\
\hline Maynard-Smith 2014 & Systematic review \\
\hline Miller 2011 & Includes both adults and children or no information about age of enrolment \\
\hline Miotto 2012 & Treatment monitoring \\
\hline Mntonintshi 2017 & Data insufficient for $2 \times 2$ table \\
\hline Modi 2016 & Xpert was not the index test \\
\hline Mokaddas 2016 & Abstract \\
\hline More 2017 & Data insufficient for $2 \times 2$ table \\
\hline Morozova 2016 & Abstract \\
\hline Moure 2012 & This study evaluated Xpert MTB/RIF for the diagnosis of extrapulmonary TB \\
\hline Mukherjee 2017 & Reference standard not satisfied \\
\hline Mulder 2017 & Xpert was not the index test \\
\hline Muñoz 2013 & Study on patient impact \\
\hline Myneedu 2014 & Includes both adults and children or no information about age of enrolment \\
\hline Naidoo 2016 & Data insufficient for $2 \times 2$ table \\
\hline Narasimooloo 2012 & Study on patient impact \\
\hline Ng 2018 & Case-control study \\
\hline Nguyen 2018 & Includes both adults and children or no information about age of enrolment \\
\hline Ngwira 2017 & Abstract \\
\hline Nhu 2013 & This study evaluated Xpert MTB/RIF for the diagnosis of TB in children \\
\hline Nicol 2011 & This study evaluated Xpert for the diagnosis of TB in children \\
\hline Ninan 2016 & Xpert was not the index test \\
\hline Nosova $2013 b$ & Duplicate data; same study as Nosova 2013a. Nosova 2013b is written in Russian \\
\hline Ntinginya 2012 & Active case finding, not a diagnostic test accuracy study \\
\hline O'Grady 2012 & $\begin{array}{l}\text { This study evaluated Xpert MTB/RIF in patients able to produce sputum, irrespective of admission } \\
\text { diagnosis, not presumed TB patients }\end{array}$ \\
\hline Omrani 2014 & Not a diagnostic accuracy study \\
\hline
\end{tabular}




\begin{tabular}{|c|c|}
\hline Study & Reason for exclusion \\
\hline Opota 2016 & Includes both adults and children or no information about age of enrolment \\
\hline Osman 2014 & Case-control study \\
\hline Ou 2015 & Includes both adults and children or no information about age of enrolment \\
\hline Ozkutuk 2014 & Includes both adults and children or no information about age of enrolment \\
\hline Pandey P 2017 & Includes both adults and children or no information about age of enrolment \\
\hline Pandey S 2017 & Includes both adults and children or no information about age of enrolment \\
\hline Parcell 2017 & Includes both adults and children or no information about age of enrolment \\
\hline Patil 2014 & Case report \\
\hline Patil 2017 & Reference standard not satisfied \\
\hline Peter 2012 & This study evaluated Xpert MTB/RIF for the diagnosis of extrapulmonary TB \\
\hline Peter 2013 & Data insufficient for $2 \times 2$ table \\
\hline Peter 2015 & Duplicate data; study was nested in Theron 2014a \\
\hline Rachow 2012 & This study evaluated Xpert for the diagnosis of TB in children \\
\hline Rahman 2016 & Not a diagnostic accuracy study \\
\hline Raizada 2015 & Not a diagnostic accuracy study \\
\hline Ramamurthy 2016 & Data insufficient for $2 \times 2$ table \\
\hline Ramirez 2014 & Not a diagnostic accuracy study \\
\hline Reechaipichitkul 2016 & Duplicate data; more participants were included in Reechaipichitkul 2017 \\
\hline Reed 2016 & Xpert was not the index test \\
\hline Rees 2018 & Impact study \\
\hline Rossato 2018 & Study design unclear, possibly case-control \\
\hline Rufai 2014 & Data insufficient for $2 \times 2$ table \\
\hline Ruiz 2017 & Xpert was not the index test \\
\hline Sachdeva 2015 & Not a diagnostic accuracy study \\
\hline Saeed 2017 & Data insufficient for $2 \times 2$ table \\
\hline Sanchez-Padilla 2015 & Not a diagnostic accuracy study \\
\hline Sauzullo 2016 & Includes both adults and children or no information about age of enrolment \\
\hline Shah 2014 & Case-control study \\
\hline
\end{tabular}




\begin{tabular}{|c|c|}
\hline Study & Reason for exclusion \\
\hline Shenai 2013 & Data insufficient for $2 \times 2$ table \\
\hline Shilpa 2017 & Reference standard not satisfied \\
\hline Smith 2014 & Not a diagnostic accuracy study \\
\hline Somashekar 2014 & Reference standard not satisfied \\
\hline Somily 2016 & Includes both pulmonary and extrapulmonary specimens combined \\
\hline Strydom 2015 & Case-control study \\
\hline Sureshbabu 2016 & Reference standard not satisfied \\
\hline Tadesse $2016 b$ & Abstract \\
\hline Tahseen 2016 & Drug resistance survey \\
\hline Tan 2017 & Xpert was not the index test \\
\hline Taylor 2012 & This study evaluated Xpert for the diagnosis of extrapulmonary TB \\
\hline Teo 2011 & Includes both adults and children or no information about age of enrolment \\
\hline Theron 2012 & Treatment monitoring \\
\hline Theron 2014b & Duplicate data set for Theron 2014a with a different aim \\
\hline Theron 2016 & $\begin{array}{l}\text { Duplicate data. Author reported that this study overlaps with the Theron } 2014 \text { a and can be exclud- } \\
\text { ed }\end{array}$ \\
\hline Theron 2018 & Screening study \\
\hline Thibbadee 2016 & Abstract \\
\hline Thit 2017 & Xpert was not the index test \\
\hline To 2017 & Abstract \\
\hline Tortoli 2012 & This study evaluated Xpert MTB/RIF for the diagnosis of extrapulmonary TB \\
\hline Ullah 2016 & Includes both adults and children or no information about age of enrolment \\
\hline Ullah 2017 & Includes both adults and children or no information about age of enrolment \\
\hline Vadwai 2011 & This study evaluated Xpert MTB/RIF for the diagnosis of extrapulmonary TB \\
\hline Van Kampen 2015 & Includes both adults and children or no information about age of enrolment \\
\hline Van Rie 2011 & Case report \\
\hline Walters 2012 & This study evaluated Xpert MTB/RIF for the diagnosis of TB in children \\
\hline Walusimbi 2013b & Systematic review \\
\hline
\end{tabular}




\begin{tabular}{|c|c|}
\hline Study & Reason for exclusion \\
\hline Wang 2015 & Systematic review \\
\hline Wang 2016 & Includes both adults and children or no information about age of enrolment \\
\hline Williamson 2012a & Case-control study \\
\hline Wood 2012 & This study evaluated Xpert MTB/RIF for the diagnosis of extrapulmonary TB \\
\hline Xie 2017 & Xpert was not the index test \\
\hline Yadav 2017 & Includes both adults and children or no information about age of enrolment \\
\hline Yan 2016 & Systematic review \\
\hline Zar 2012 & This study evaluated Xpert MTB/RIF for the diagnosis of TB in children \\
\hline Zemlyansky 2016 & Includes both adults and children or no information about age of enrolment \\
\hline
\end{tabular}

\section{Characteristics of ongoing studies [ordered by study ID]}

\section{Koenig 2018}

Trial name or title

\section{Trial name or title}

Target condition and refer-
ence standard(s)

A trial of same-day testing and treatment to improve outcomes among symptomatic patients newly diagnosed with HIV

Tuberculosis, HIV/AIDS
Spot and early-morning Xpert Ultra results and chest $x$-ray, as single and as combined tests, with liquid culture as reference standard

\begin{tabular}{ll}
\hline Starting date & 16 May 2017 \\
\hline Contact information & Serena P Koenig, MD, skoenig@bwh.harvard.edu \\
\hline Notes & ClinicalTrials.gov Identifier: NCT03154320 \\
\hline
\end{tabular}

\section{Reid 2018}

\begin{tabular}{ll}
\hline Trial name or title & Achieving tuberculosis control In Zambia \\
\hline $\begin{array}{l}\text { Target condition and reference } \\
\text { standard(s) }\end{array}$ & Tuberculosis \\
\hline Index and comparator tests & $\begin{array}{l}\text { Comparison of two diagnostic tools (chest-xray with computer-assisted diagnosis versus C-reac- } \\
\text { tive protein) and Xpert Ultra for active community-based tuberculosis case detection }\end{array}$ \\
\hline Starting date & 13 April 2018 \\
\hline Contact information & Stewart Reid, MD, MPH, stewart.reid@cidrz.org \\
\hline
\end{tabular}


Reid 2018 (Continued)

Notes ClinicalTrials.gov Identifier: NCT03497195

Theron 2018a

\begin{tabular}{ll}
\hline Trial name or title & $\begin{array}{l}\text { Improving tuberculosis diagnosis and treatment through Basic, Applied and health systems Re- } \\
\text { search (BAR) }\end{array}$ \\
\hline $\begin{array}{l}\text { Target condition and reference } \\
\text { standard(s) }\end{array}$ & Tuberculosis \\
\hline Index and comparator tests & $\begin{array}{l}\text { Xpert Ultra point-of-care testing compared to the standard of care tuberculosis testing at a } \\
\text { centralised facility }\end{array}$ \\
\hline Starting date & 29 November 2017 \\
\hline Contact information & Grant Theron, PhD. gtheron@sun.ac.za \\
\hline Notes & ClinicalTrials.gov Identifier: NCT03356925 \\
\hline
\end{tabular}

Theron 2018b

\begin{tabular}{ll}
\hline Trial name or title & Xpert Ultra and Xpert HIV-VL in people living with HIV (UltraHIV) \\
\hline Target condition and reference standard(s) & Tuberculosis, HIV/AIDS \\
\hline Index and comparator tests & Impact study \\
\hline Starting date & 15 June 2017 \\
\hline Contact information & Grant Theron, PhD. gtheron@sun.ac.za \\
\hline Notes & ClinicalTrials.gov Identifier: NCT03187964 \\
\hline
\end{tabular}

\section{Zhang 2018}

\begin{tabular}{ll}
\hline Trial name or title & $\begin{array}{l}\text { Diagnostic accuracy of Xpert MTB/RIF Ultra for tuberculous bronchoalveolar lavage fluid in } \\
\text { HIV-infected adults: a prospective cohort study }\end{array}$ \\
\hline $\begin{array}{l}\text { Target condition and reference stan- } \\
\text { dard(s) }\end{array}$ & Tuberculosis and HIV/AIDS, MGIT \\
\hline Index and comparator tests & Xpert Ultra \\
\hline Starting date & 12 February 2018 \\
\hline Contact information & Peize Zhang, 516472422@qq.com \\
\hline Notes & WHO International Clinical Trials: Chi CTR1800014792 \\
\hline
\end{tabular}




\section{A T A}

Presented below are all the data for all of the tests entered into the review.

\section{Table Tests. Data tables by test}

\begin{tabular}{|c|c|c|}
\hline Test & No. of studies & $\begin{array}{l}\text { No. of partici- } \\
\text { pants }\end{array}$ \\
\hline 2 Xpert Ultra for detection of PTB & 1 & 1439 \\
\hline 3 Smear-positive, Xpert MTB/RIF & 53 & 4943 \\
\hline 4 Smear-positive, Xpert MTB/RIF, direct comparison Xpert MTB/RIF vs Xpert Ultra & 1 & 323 \\
\hline 5 Smear-positive, Xpert Ultra, direct comparison Xpert MTB/RIF vs Xpert Ultra & 1 & 323 \\
\hline 6 Smear-negative, Xpert MTB/RIF & 56 & 22581 \\
\hline 7 Smear-negative, Xpert MTB/RIF, direct comparison Xpert MTB/RIF vs Xpert Ultra & 1 & 1111 \\
\hline 8 Smear-negative, Xpert Ultra, direct comparison Xpert MTB/RIF vs Xpert Ultra & 1 & 1111 \\
\hline 9 HIV-negative, Xpert MTB/RIF & 18 & 5118 \\
\hline 10 HIV-positive, Xpert MTB/RIF & 30 & 9593 \\
\hline 11 HIV-negative, within study comparisons & 14 & 4681 \\
\hline 12 HIV-positive, within study comparisons & 14 & 4663 \\
\hline 13 HIV-negative, Xpert MTB/RIF, direct comparison Xpert MTB/RIF vs Xpert Ultra & 1 & 483 \\
\hline 15 HIV-positive, Xpert MTB/RIF, direct comparison Xpert MTB/RIF vs Xpert Ultra & 1 & 432 \\
\hline 16 HIV-positive, Xpert Ultra, direct comparison Xpert MTB/RIF vs Xpert Ultra & 1 & 432 \\
\hline 17 Xpert MTB/RIF for detection of rifampicin resistance & 57 & 8287 \\
\hline 18 Xpert Ultra for detection of rifampicin resistance & 1 & 551 \\
\hline
\end{tabular}

Test 1. Xpert MTB/RIF for detection of pulmonary tuberculosis (PTB).

Test 2. Xpert Ultra for detection of PTB. 
Test 3. Smear-positive, Xpert MTB/RIF.

Test 4. Smear-positive, Xpert MTB/RIF, direct comparison Xpert MTB/RIF vs Xpert Ultra.

Test 5. Smear-positive, Xpert Ultra, direct comparison Xpert MTB/RIF vs Xpert Ultra.

Test 6. Smear-negative, Xpert MTB/RIF.

Test 7. Smear-negative, Xpert MTB/RIF, direct comparison Xpert MTB/RIF vs Xpert Ultra.

Test 8. Smear-negative, Xpert Ultra, direct comparison Xpert MTB/RIF vs Xpert Ultra.

Test 9. HIV-negative, Xpert MTB/RIF.

Test 10. HIV-positive, Xpert MTB/RIF.

Test 11. HIV-negative, within study comparisons.

Test 12. HIV-positive, within study comparisons.

Test 13. HIV-negative, Xpert MTB/RIF, direct comparison Xpert MTB/RIF vs Xpert Ultra.

Test 14. HIV-negative, Xpert Ultra, direct comparison Xpert MTB/RIF vs Xpert Ultra.

Test 15. HIV-positive, Xpert MTB/RIF, direct comparison Xpert MTB/RIF vs Xpert Ultra. 
Test 16. HIV-positive, Xpert Ultra, direct comparison Xpert MTB/RIF vs Xpert Ultra.

Test 17. Xpert MTB/RIF for detection of rifampicin resistance.

Test 18. Xpert Ultra for detection of rifampicin resistance.

\section{ADDITIONAL TABLES}

Table 1. Xpert MTB/RIF for detection of pulmonary tuberculosis and rifampicin resistance

\begin{tabular}{|c|c|c|c|c|}
\hline Type of analysis (number of studies; participants) & $\begin{array}{l}\text { Median } \\
\text { pooledsen- } \\
\text { sitivity } \\
(95 \% \text { Crl })\end{array}$ & $\begin{array}{l}\text { Median } \\
\text { pooled- } \\
\text { specificity } \\
(95 \% \text { Crl) }\end{array}$ & $\begin{array}{l}\text { Median pre- } \\
\text { dicted sen- } \\
\text { sitivity } \\
\text { (95\% Crl) }\end{array}$ & $\begin{array}{l}\text { Median pre- } \\
\text { dicted speci- } \\
\text { ficity } \\
\text { (95\% Crl) }\end{array}$ \\
\hline $\begin{array}{l}\text { Xpert MTB/RIF sensitivity and specificity for detection of PTB, all } \\
\text { studies }^{a}(85 ; 41,965)\end{array}$ & $\begin{array}{l}85 \%(82 \text { to } \\
87)\end{array}$ & $\begin{array}{l}98 \%(97 \text { to } \\
98)\end{array}$ & $\begin{array}{l}85 \%(52 \text { to } \\
97)\end{array}$ & $\begin{array}{l}98 \%(76 \text { to } \\
100)\end{array}$ \\
\hline $\begin{array}{l}\text { Xpert MTB/RIF sensitivity and specificity for detection of PTB, } \\
\text { studies with unselected participants }(70 ; 37,237)\end{array}$ & $\begin{array}{l}85 \%(82 \text { to } \\
88)\end{array}$ & $\begin{array}{l}98 \% \text { (97 to } \\
98)\end{array}$ & $\begin{array}{l}85 \%(56 \text { to } \\
96)\end{array}$ & $\begin{array}{l}98 \%(78 \text { to } \\
100)\end{array}$ \\
\hline $\begin{array}{l}\text { Xpert MTB/RIF sensitivity and specificity for detection of ri- } \\
\text { fampicin resistance }(48 ; 8020)\end{array}$ & $\begin{array}{l}96 \% \text { (94 to } \\
97)\end{array}$ & $\begin{array}{l}98 \% \text { (98 to } \\
99)\end{array}$ & $\begin{array}{l}96 \% \text { (86 to } \\
99)\end{array}$ & $\begin{array}{l}98 \% \text { (89 to } \\
100)\end{array}$ \\
\hline
\end{tabular}

Abbreviations: Crl: credible interval; PTB: pulmonary tuberculosis.

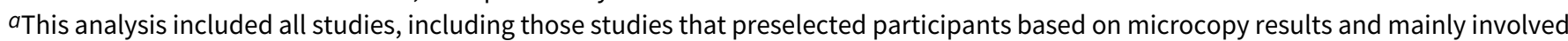
participants who had received previous tuberculosis treatment.

Table 2. Xpert MTB/RIF for detection of pulmonary tuberculosis, investigations of heterogeneity

\begin{tabular}{lllll}
\hline $\begin{array}{l}\text { Type of analysis (number of studies; partic- } \\
\text { ipants) }\end{array}$ & $\begin{array}{l}\text { Median pooled } \\
\text { sensitivity } \\
\text { (95\% CrI) }\end{array}$ & $\begin{array}{l}\text { Median pooled } \\
\text { specificity } \\
\text { (95\% CrI) }\end{array}$ & $\begin{array}{l}\text { Median predicted } \\
\text { sensitivity } \\
\text { (95\% Crl) }\end{array}$ & $\begin{array}{l}\text { Median predicted } \\
\text { specificity } \\
\text { (95\% CrI) }\end{array}$ \\
\hline Xpert MTB/RIF accuracy for tuberculosis detection in clinical subgroups & & & \\
\hline Smear positive $(45 ; 4064)^{a}$ & $98 \%(97$ to 98$)$ & $\begin{array}{l}\text { Could not deter- } \\
\text { mine }\end{array}$ & $98 \%(89$ to 100) & $\begin{array}{l}\text { Could not deter- } \\
\text { mine }\end{array}$ \\
\hline Smear negative $(45 ; 18,962)^{a}$ & $67 \%(62$ to 72$)$ & $98 \%(98$ to 99$)$ & $67 \%(37$ to 88$)$ & $98 \%(80$ to 100$)$ \\
\hline HIV negative $(14 ; 3866)^{a}$ & $88 \%(83$ to 92$)$ & $98 \%(97$ to 99) & $88 \%(71$ to 96) & $98 \%(92$ to 100) \\
\hline HIV positive $(14 ; 4664)^{a}$ & $81 \%(75$ to 86$)$ & $98 \%(97$ to 99$)$ & $81 \%(59$ to 93) & $98 \%(92$ to 100) \\
\hline
\end{tabular}

Xpert MTB/RIF accuracy for tuberculosis detection based on percentage of participants with a history of previous tuberculosis

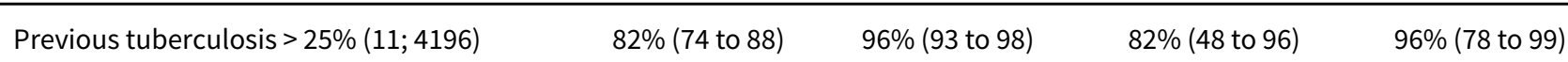


Table 2. Xpert MTB/RIF for detection of pulmonary tuberculosis, investigations of heterogeneity (Continued)
Previous tuberculosis $\leq 25 \%(16 ; 8205)$
$81 \%(72$ to 87$)$
98\% (97 to 99)
$81 \%$ (39 to 97 )
$98 \%$ (90 to 100$)$

\begin{tabular}{|c|c|c|c|c|}
\hline High tuberculosis burden $=$ Yes $(39 ; 21,965)^{\mathrm{b}}$ & $86 \%$ (82 to 89$)$ & $97 \%$ (95 to 98$)$ & $86 \%(57$ to 96$)$ & $97 \%(71$ to 100$)$ \\
\hline High tuberculosis burden $=$ No $(33 ; 15,272)^{b}$ & $85 \%$ (81 to 89$)$ & $99 \%$ (98 to 99$)$ & $85 \%$ (55 to 96$)$ & $99 \%$ (89 to 100$)$ \\
\hline \multicolumn{5}{|c|}{ Xpert MTB/RIF accuracy for tuberculosis detection by TB/HIV burden ${ }^{a}$} \\
\hline High TB/HIV burden = Yes $(42 ; 24,412)^{b}$ & $83 \%$ (80 to 87$)$ & $97 \%$ (95 to 98$)$ & $84 \%$ (51 to 96$)$ & $97 \%(74$ to 100$)$ \\
\hline High TB/HIV burden = No $(30 ; 12,825)^{b}$ & $88 \%(84$ to 90$)$ & $99 \%$ (98 to 99$)$ & $88 \%(67$ to 96$)$ & $99 \%(86$ to 100$)$ \\
\hline \multicolumn{5}{|c|}{ Xpert MTB/RIF accuracy for tuberculosis detection by setting that ran the test } \\
\hline $\begin{array}{l}\text { Xpert run at point of care or in a peripheral } \\
\text { setting }(10 ; 5816)\end{array}$ & $83 \%$ (75 to 89$)$ & $97 \%$ (93 to 99$)$ & $83 \%$ (52 to 96$)$ & $97 \%$ (66 to 100$)$ \\
\hline $\begin{array}{l}\text { Central or intermediate laboratory }(60 ; \\
31,421)\end{array}$ & $85 \%$ (83 to 88$)$ & $98 \%$ (97 to 98$)$ & $85 \%$ (57 to 96 ) & $98 \%$ (80 to 100$)$ \\
\hline
\end{tabular}

\section{Xpert MTB/RIF accuracy for tuberculosis detection by median tuberculosis prevalence}

\begin{tabular}{|c|c|c|c|c|}
\hline Prevalence $>26 \%(35 ; 17,983)$ & $89 \%$ (87 to 91 ) & $96 \%$ (94 to 97$)$ & $89 \%$ (69 to 97 ) & $96 \%$ (72 to 100$)$ \\
\hline Prevalence $\leq 26 \%(35 ; 19,254)$ & $79 \%$ (75 to 83$)$ & $99 \%$ (98 to 99$)$ & $79 \%$ (51 to 93$)$ & $99 \%$ (89 to 100$)$ \\
\hline
\end{tabular}

Abbreviations: Crl: credible interval; HIV: human immunodeficiency virus; TB: tuberculosis.

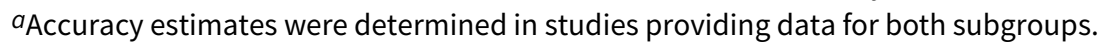

bSubstudies from Boehme 2010 and Boehme 2011 contributed to both tuberculosis burden categories.

Table 3. Xpert MTB/RIF for detection of rifampicin resistance, investigations of heterogeneity

\begin{tabular}{lllll}
\hline $\begin{array}{l}\text { Type of analysis (Number of studies; par- } \\
\text { ticipants) }\end{array}$ & $\begin{array}{l}\text { Median pooled } \\
\text { sensitivity }\end{array}$ & $\begin{array}{l}\text { Median pooled } \\
\text { specificity }\end{array}$ & $\begin{array}{l}\text { Median predicted } \\
\text { sensitivity }\end{array}$ & $\begin{array}{l}\text { Median predicted } \\
\text { specificity }\end{array}$ \\
& $(95 \% \mathrm{Crl})$ & $(95 \% \mathrm{Crl})$ & $(95 \% \mathrm{Crl})$ & $(95 \% \mathrm{Crl})$
\end{tabular}

\begin{tabular}{|c|c|c|c|c|}
\hline \multicolumn{5}{|c|}{ Xpert MTB/RIF accuracy for rifampicin resistance detection by MDR-TB burden } \\
\hline High MDR-TB burden = Yes $(24 ; 5553)$ & $95 \%$ (93 to 97 ) & $98 \%$ (96 to 99$)$ & $95 \%$ (85 to 99$)$ & $98 \%(85$ to 100$)$ \\
\hline High MDR-TB burden = No $(25 ; 2467)$ & $97 \%$ (93 to 99$)$ & $99 \%$ (98 to 99$)$ & $97 \%$ (76 to 100$)$ & $99 \%(95$ to 100$)$ \\
\hline \multicolumn{5}{|c|}{ Xpert MTB/RIF accuracy for rifampicin resistance detection by history of previous tuberculosis treatment } \\
\hline $\begin{array}{l}\text { Previously-treated tuberculosis }{ }^{a}=\text { Yes }(7 ; \\
\text { 1062) }\end{array}$ & $98 \%(94$ to 99$)$ & $97 \%$ (93 to 99) & $98 \%$ (87 to 100$)$ & $97 \%(81$ to 100$)$ \\
\hline $\begin{array}{l}\text { Previously-treated tuberculosis }=\text { No }(41, \\
6958)\end{array}$ & $95 \%$ (93 to 97 ) & $99 \%$ (98 to 99$)$ & $95 \%$ (86 to 99$)$ & $98 \%$ (91 to 100$)$ \\
\hline
\end{tabular}

Xpert MTB/RIF accuracy for detection of rifampicin resistance by median tuberculosis prevalence 
Table 3. Xpert MTB/RIF for detection of rifampicin resistance, investigations of heterogeneity (Continued)

\begin{tabular}{lllll} 
Prevalence $>11 \%(24 ; 5505)$ & $96 \%(94$ to 97$)$ & $97 \%(96$ to 98$)$ & $96 \%(87$ to 99$)$ & $97 \%(88$ to 99$)$ \\
\hline Prevalence $\leq 11 \%(24 ; 2515)$ & $94 \%(89$ to 97$)$ & $99 \%(99$ to 100$)$ & $94 \%(80$ to 99$)$ & $99 \%(96$ to 100$)$
\end{tabular}

Abbreviations: Crl: credible interval; MDR-TB: multidrug-resistant tuberculosis.

aStudies with high percentages of participants previously treated for tuberculosis.

Table 4. Sensitivity analyses, Xpert MTB/RIF

\begin{tabular}{|c|c|c|c|c|}
\hline Type of analysis (Number of studies; participants) & $\begin{array}{l}\text { Median } \\
\text { pooled } \\
\text { sensitivity } \\
(95 \% \mathrm{Crl})\end{array}$ & $\begin{array}{l}\text { Median } \\
\text { pooled } \\
\text { specificity } \\
(95 \% \text { Crl })\end{array}$ & $\begin{array}{l}\text { Median } \\
\text { predicted } \\
\text { sensitivity } \\
(95 \% \text { Crl) }\end{array}$ & $\begin{array}{l}\text { Median } \\
\text { predicted } \\
\text { specificity } \\
(95 \% \text { Crl) }\end{array}$ \\
\hline $\begin{array}{l}\text { Xpert MTB/RIF sensitivity and specificity for tuberculosis detection in } \\
\text { studies with unselected patients }(70 ; 37,237)\end{array}$ & $\begin{array}{l}85 \%(82 \text { to } \\
88)\end{array}$ & $\begin{array}{l}98 \% \text { (97 to } \\
98)\end{array}$ & $\begin{array}{l}85 \%(56 \text { to } \\
96)\end{array}$ & $\begin{array}{l}98 \%(78 \text { to } \\
100)\end{array}$ \\
\hline $\begin{array}{l}\text { Studies that explicitly represented the use of the index test for the diag- } \\
\text { nosis of individuals with signs and symptoms of tuberculosis (presump- } \\
\text { tive tuberculosis) }(62 ; 33,844)\end{array}$ & $\begin{array}{l}86 \%(84 \text { to } \\
89)\end{array}$ & $\begin{array}{l}98 \% \text { (97 to } \\
98)\end{array}$ & $\begin{array}{l}86 \%(54 \text { to } \\
97)\end{array}$ & $\begin{array}{l}98 \%(78 \text { to } \\
100)\end{array}$ \\
\hline $\begin{array}{l}\text { Studies where a single specimen yielded a single Xpert MTB/RIF result } \\
\text { for a given participant }(53 ; 27,306)\end{array}$ & $\begin{array}{l}85 \%(81 \text { to } \\
87)\end{array}$ & $\begin{array}{l}98 \% \text { (97 to } \\
98)\end{array}$ & $\begin{array}{l}85 \%(50 \text { to } \\
97)\end{array}$ & $\begin{array}{l}97 \%(80 \text { to } \\
100)\end{array}$ \\
\hline Studies that included only untreated participants $(36 ; 15,502)$ & $\begin{array}{l}82 \%(79 \text { to } \\
86)\end{array}$ & $\begin{array}{l}98 \% \text { (98 to } \\
99)\end{array}$ & $\begin{array}{l}83 \%(52 \text { to } \\
96)\end{array}$ & $\begin{array}{l}98 \%(90 \text { to } \\
100)\end{array}$ \\
\hline Studies that used liquid culture as the reference standard $(24 ; 12,548)$ & $\begin{array}{l}83 \%(78 \text { to } \\
88)\end{array}$ & $\begin{array}{l}97 \% \text { (95 to } \\
98)\end{array}$ & $\begin{array}{l}83 \%(48 \text { to } \\
97)\end{array}$ & $\begin{array}{l}97 \%(65 \text { to } \\
100)\end{array}$ \\
\hline $\begin{array}{l}\text { Studies where consecutive or random participants were selected (52; } \\
28,633)\end{array}$ & $\begin{array}{l}84 \%(80 \text { to } \\
87)\end{array}$ & $\begin{array}{l}98 \% \text { (97 to } \\
98)\end{array}$ & $\begin{array}{l}84 \%(50 \text { to } \\
96)\end{array}$ & $\begin{array}{l}98 \%(78 \text { to } \\
100)\end{array}$ \\
\hline Studies where the reference standard was blinded $(56 ; 31,228)$ & $\begin{array}{l}84 \%(81 \text { to } \\
87)\end{array}$ & $\begin{array}{l}97 \%(96 \text { to } \\
98)\end{array}$ & $\begin{array}{l}85 \%(50 \text { to } \\
97)\end{array}$ & $\begin{array}{l}97 \% \text { (77 to } \\
100)\end{array}$ \\
\hline Studies using fresh specimens $(56 ; 29,090)$ & $\begin{array}{l}86 \% \text { (83 to } \\
88)\end{array}$ & $\begin{array}{l}98 \% \text { (97 to } \\
98)\end{array}$ & $\begin{array}{l}86 \% \text { (50 to } \\
97)\end{array}$ & $\begin{array}{l}98 \% \text { ( } 75 \text { to } \\
100)\end{array}$ \\
\hline Studies that accounted for all participants in the analysis $(59 ; 27,128)$ & $\begin{array}{l}85 \% \text { (82 to } \\
88)\end{array}$ & $\begin{array}{l}98 \% \text { (97 to } \\
98)\end{array}$ & $\begin{array}{l}85 \% \text { (49 to } \\
97 \text { ) }\end{array}$ & $\begin{array}{l}98 \% \text { (76 to } \\
100)\end{array}$ \\
\hline Excluding Boehme 2010 and Boehme 2011 (68; 31889) & $\begin{array}{l}85 \% \text { (82 to } \\
87 \text { ) }\end{array}$ & $\begin{array}{l}98 \% \text { (97 to } \\
98 \text { ) }\end{array}$ & $\begin{array}{l}85 \% \text { (55 to } \\
96 \text { ) }\end{array}$ & $\begin{array}{l}98 \% \text { (77 to } \\
100)\end{array}$ \\
\hline
\end{tabular}

Abbreviations: Crl: credible interval. 


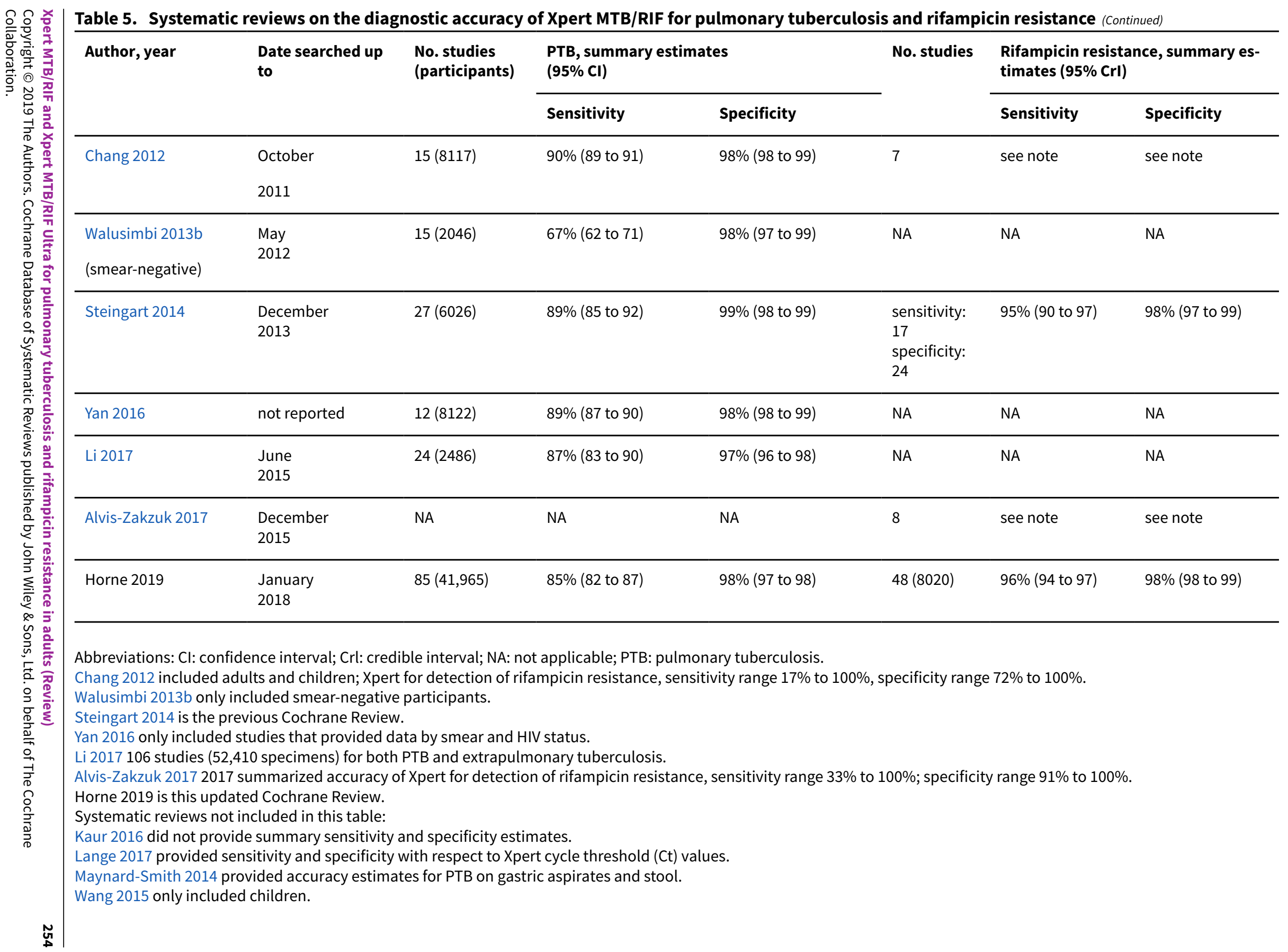




\section{AP PEN DICES}

\section{Appendix 1. Search strategy}

MEDLINE (OVID) and Embase (OVID)

1. (tuberculosis or TB).tw

limit 1 to $y r=" 2007$-Current"

2. Mycobacterium tuberculosis/

limit 2 to $y r=" 2007$-Current"

3. Tuberculosis, Multidrug-Resistant/ or Tuberculosis/ or Tuberculosis, Pulmonary/

limit 3 to yr="2007 -Current"

4. 1 or 2 or 3

5. (Xpert or GeneXpert or cepheid or( near ${ }^{\star}$ patient)). tw.

limit 4 to $y r=" 2007$-Current"

4 and 5

\section{Web of Knowledge (SCl-expanded, SSCI, Conference Proceedings science, BIOSIS previews)}

(tuberculosis OR TB OR mycobacterium) (topic) AND (Xpert OR Genexpert OR cepheid) (topic)

\section{LILACS}

(tuberculosis OR TB OR mycobacterium) (Words) AND (xpert OR Genexpert OR Cepheid) (Words)

\section{SCOPUS}

(tuberculosis OR TB OR mycobacterium) (title, abstract, keywords) AND (xpert OR Genexpert OR Cepheid) (title, abstract, keywords)

\section{Appendix 2. Boehme 2010 and Boehme 2011, multicentre studies}

A. Boehme 2010 and Boehme 2011, multicentre studies, Xpert MTB/RIF for detection of pulmonary tuberculosis

\begin{tabular}{|c|c|c|c|c|c|}
\hline Study & Site & $\begin{array}{l}\text { True posi- } \\
\text { tive }\end{array}$ & $\begin{array}{l}\text { False pos- } \\
\text { itive }\end{array}$ & $\begin{array}{l}\text { False } \\
\text { negative }\end{array}$ & $\begin{array}{l}\text { True nega- } \\
\text { tive }\end{array}$ \\
\hline Boehme 2010a & Azerbaijan & 123 & 8 & 24 & 91 \\
\hline Boehme 2010b & Peru & 201 & 1 & 8 & 105 \\
\hline Boehme 2010c & South Africa, Cape Town & 136 & 9 & 10 & 188 \\
\hline Boehme 2010d & South Africa, Durban & 36 & 7 & 7 & 257 \\
\hline Boehme 2010e & India & 179 & 1 & 8 & 40 \\
\hline Boehme 2011a & Azerbaijan & 203 & 4 & 26 & 303 \\
\hline Boehme 2011a,b & Peru & 171 & 3 & 6 & 825 \\
\hline Boehme 2011c & South Africa & 201 & 2 & 32 & 669 \\
\hline Boehme 2011d & Uganda & 121 & 0 & 24 & 144 \\
\hline
\end{tabular}

Xpert MTB/RIF and Xpert MTB/RIF Ultra for pulmonary tuberculosis and rifampicin resistance in adults (Review) 
(Continued)

\begin{tabular}{llrr} 
Boehme 2011e & India & 101 & 0 \\
\hline Boehme 2011f & The Philippines & 1636 & 5 \\
\hline
\end{tabular}

B. Boehme 2010 and Boehme 2011, multicentre studies, Xpert MTB/RIF for detection of rifampicin resistance

\begin{tabular}{|c|c|c|c|c|c|}
\hline Study & Site & $\begin{array}{l}\text { True pos- } \\
\text { itive }\end{array}$ & $\begin{array}{l}\text { False pos- } \\
\text { itive }\end{array}$ & $\begin{array}{l}\text { False } \\
\text { negative }\end{array}$ & $\begin{array}{l}\text { True nega- } \\
\text { tive }\end{array}$ \\
\hline Boehme 2010a & Azerbaijan & 47 & 4 & 2 & 90 \\
\hline Boehme 2010b & Peru & 16 & 3 & 0 & 190 \\
\hline Boehme 2010c & South Africa, Cape Town & 15 & 0 & 1 & 126 \\
\hline Boehme 2010d & South Africa, Durban & 3 & 0 & 0 & 38 \\
\hline Boehme 2010e & India & 119 & 3 & 2 & 61 \\
\hline Boehme 2011a & Azerbaijan & 47 & 1 & 3 & 160 \\
\hline Boehme 2011b & Peru & 22 & 1 & 1 & 161 \\
\hline Boehme 2011c & South Africa & 9 & 3 & 1 & 175 \\
\hline Boehme 2011d & Uganda & 1 & 1 & 2 & 112 \\
\hline Boehme 2011e & India & 8 & 2 & 2 & 91 \\
\hline Boehme $2011 \mathrm{f}$ & The Philippines & 149 & 6 & 5 & 97 \\
\hline
\end{tabular}

Footnotes: In the 2014 Cochrane Review, for multicentre studies, the study-naming scheme uniquely identified multiple study centres from within each study (for example, Boehme 2010a; Boehme 2010b), each of which reported data separately for a distinct population at a given study site.

\section{Appendix 3. Data extraction form}

\section{ID}

ID substudy (for study centres: a, b, c, etc)

First author

Corresponding author \& email

$\begin{array}{ll}\text { Was author contacted? } & 1-\text { Yes } \\ & 2-\text { No } \\ \text { If yes, dates(s) }\end{array}$


Year (of publication)

Year (study start date)

\begin{tabular}{ll}
\hline Language & 1 - English \\
& 2 - Other \\
& If other, specify: \\
\hline
\end{tabular}

\section{Study details}

Country where study was conducted

\begin{tabular}{|c|c|}
\hline \multirow[t]{7}{*}{ Country World Bank Classification } & 1 - Low income \\
\hline & 2 - Middle income \\
\hline & 3 - High income \\
\hline & 4 - Low and high income \\
\hline & 5 - Low and middle income \\
\hline & 6 - Low, middle, and high \\
\hline & 7 - Other combination, describe \\
\hline \multirow[t]{4}{*}{ Purpose of testing as described in the study } & 1 - Diagnosis \\
\hline & 2 - Screening in HIV-positive people \\
\hline & 9 - Could not tell \\
\hline & Study states: \\
\hline \multirow[t]{3}{*}{ Objective of study } & 1 - Detection of PTB only \\
\hline & 2 - Detection of rifampicin resistance only \\
\hline & 3 - Both, detection of PTB and rifampicin resistance \\
\hline \multirow[t]{6}{*}{ Study design } & 1 - Randomized controlled trial \\
\hline & 2 - Cross-sectional \\
\hline & 3 - Cohort \\
\hline & 4 - Other, specify \\
\hline & 9 - Could not tell \\
\hline & If other, describe: \\
\hline
\end{tabular}

\section{Ila. Questions about pre-selection during enrolment}

Were patients pre-selected based upon microscopy results?

1 - Yes

2 - No 


\begin{tabular}{|c|c|}
\hline Did study include exclusively retreatment patients & $1-$ Yes \\
\hline upon enrolment? (for example, patients who previously received & 2 - No \\
\hline first-line drugs and those with nonconverting & 9 - Unknown/NR \\
\hline
\end{tabular}

\begin{tabular}{|c|c|}
\hline Participant selection & $\begin{array}{l}\text { 1-Consecutive } \\
2 \text { - Random } \\
3 \text { - Convenience } \\
7 \text { - Other } \\
9 \text { - Unknown/NR }\end{array}$ \\
\hline Direction of study data collection & $\begin{array}{l}1 \text { - Prospective } \\
2 \text { - Retrospective } \\
9 \text { - Unknown/NR }\end{array}$ \\
\hline Number included after recruitment by inclusion and exclusion criteria & $9-$ Unknown/NR \\
\hline Number included in analysis (\# recruited - \# withdrawals) & $9-$ Unknown/NR \\
\hline Unit of analysis & $\begin{array}{l}1 \text { - Patient (with a single Xpert per patient) } \\
2 \text { - Specimen (there are more specimens than patients) } \\
9 \text { - Unknown/NR } \\
\text { Describe as in paper, if unclear: }\end{array}$ \\
\hline
\end{tabular}

Comments about study design

\section{Patient characteristics and setting}

\section{Presenting signs and symptoms}

$\begin{array}{ll}\begin{array}{l}\text { Did the study avoid inappropriate exclusions? Please list exclusions noted } \\ \text { in }\end{array} & 1 \text { - Yes } \\ \text { study, if any (for example, study includes predominantly or exclusively } & 2 \text { - No } \\ \text { smear-positive or "difficult-to-diagnose" patients) } & 9 \text { - Unknonwn/NR } \\ & \text { Describe exclusions as stated in study: }\end{array}$


(Continued)

Type of specimen (may include expectorated,

1 - Expectorated sputum

induced, bronchial alveolar lavage (BAL), tracheal aspirates)(check all that

2 - Induced sputum

apply). Assume expectorated sputum if not specifically stated.

3 - Bronchial alveolar lavage or bronchial aspirates

4 - Tracheal aspirates

6 - Other

9 - Unknown/NR

If other, describe types and record numbers:

Clinical setting; describe as written in the paper

1 - Outpatient

2 - Inpatient

3 - Both out- and in-patient

4 - Other, specify

5 - Laboratory based

9 - Unknown/NR

Describe as in paper:

Was Xpert testing performed at point of care?

(POCT is diagnostic testing that will result in a

clear and actionable management decision (e.g.

start of treatment, referral, initiation of confirmatory

test) within the same clinical encounter (e.g. same

day). POCT should be mentioned in the study as

it is unlikely if testing takes place in a central

level laboratory.

\section{1 - Yes}

2 - No

9 - Could not tell
Level of the laboratory system where Xpert tests

were performed

(Tests generally available at different laboratory

levels, though tests may overlap)

Central: Intermediate laboratory tests and culture

on liquid media and DST (1st and 2nd line

anti-tuberculosis drugs) on solid or in liquid media and LPA

on positive cultures and rapid speciation tests

Intermediate: Peripheral laboratory tests and

culture on solid media and line probe assay (LPA)

from smear positive sputum

\section{1- Central}

2 - Intermediate

3 - Peripheral

4- Other, specify

Describe as in paper: 
(Continued)

Peripheral: AFB (Ziehl-Neelsen, Auramine-rhodamine,

Auramine-O staining) and Xpert MTB/RIF

\section{Other demographics}

\begin{tabular}{|c|c|}
\hline Age (range, mean (SD), median (IQR)) & 9 - Unknown/NR \\
\hline \#\#/total and \% female & 9 - Unknown/NR \\
\hline \multirow[t]{4}{*}{ HIV status of participants } & 0 - HIV - \\
\hline & $1-\mathrm{HIV}+$ \\
\hline & 2 - Both HIV+/- \\
\hline & $9-$ Unknown/NR \\
\hline If HIV-positive participants included, what is the percentage? & $\%$ (specify numerator/denominator) \\
\hline \multirow{3}{*}{$\begin{array}{l}\text { Prior tuberculosis history: Did the study include patients with prior tuber- } \\
\text { culosis history? }\end{array}$} & 1 - Yes \\
\hline & 2 - No \\
\hline & 9 - Unknown/NR \\
\hline \multirow[t]{2}{*}{ If so, what is the percentage? } & $\%$ (specify numerator/denominator) \\
\hline & 9 - Unknown/NR (for data entry write "NR") \\
\hline \multirow{3}{*}{$\begin{array}{l}\text { Prior treatment: Did the study include patients with prior tuberculosis } \\
\text { treatment? }\end{array}$} & $1-$ Yes \\
\hline & $2-$ No \\
\hline & 9 - Unknown/NR \\
\hline \multirow[t]{2}{*}{ If so, what is the percentage? } & $\%$ (specify numerator/denominator) \\
\hline & 9 - Unknown/NR (for data entry write "NR") \\
\hline \multirow{2}{*}{$\begin{array}{l}\text { Current treatment: Were patients on treatment (defined as tuberculosis } \\
\text { drugs for }\end{array}$} & $1-$ Yes \\
\hline & $2-\mathrm{No}$ \\
\hline \multicolumn{2}{|l|}{ greater than 7 days) for the current tuberculosis episode? } \\
\hline \multicolumn{2}{|l|}{ (note: may impact culture results) } \\
\hline \multirow[t]{2}{*}{ If so, what is the percentage? } & $\%$ (specify numerator/denominator) \\
\hline & 9 - Unknown/NR (for data entry write "NR") \\
\hline
\end{tabular}

\section{Index test}

Xpert version(s) evaluated

1 - Xpert MTB/RIF only

2 - Xpert Ultra only

3 - Any combination Xpert MTB/RIF and Xpert Ultra

Xpert platform: Was Omni used? Unless Omni explicitely described, as-

1 - Yes, only Omni used for Xpert tests sume 
(Continued)

standard platform
2 - Yes, both Omni and standard platform used for Xpert tests

3 - No

1-Yes (Since Xpert is automated, we will answer 'Yes" for all

studies)

\section{Reference standard}

For tuberculosis detection, what reference standard(s) was used?

1 - Solid culture (specify 1a)

2 - Liquid culture (specify 2a)

3 - Both solid and liquid culture (specify $1 \mathrm{a}$ and $2 \mathrm{a}$ )

9 - Unknown/NR

$1 a$ - Solid culture

LJ

$7 \mathrm{H} 10$

$7 \mathrm{H} 11$

Other

9- Unknown/NR

$2 a$ - Liquid culture

MGIT 960

Other (specify):

9- Unknown/NR

For MGIT only, if more than one specimen was inoculated for

culture, were these specimens obtained on different days?

For rifampicin resistance detection, what reference standard(s)

was used?
$1-$ Yes
2 - No
8 - Not applicable
9 - Unknown/NR 
2a - Liquid culture

MGIT 960

Other (specify)

$\begin{array}{ll}\text { Tuberculosis detection: Was the reference standard result interpreted } & 1-\text { Yes } \\ & 2-\text { No } \\ \text { without knowledge of the index test result? } & 9-\text { Unknown/NR }\end{array}$

Answer yes for MGIT and LJ with species confirmation

Rifampicin resistance detection: Was the reference standard

result interpreted without knowledge of the index test result?

Answer yes for MGIT

9 - Unknown/NR

Answer yes for MGIT

\section{Specimen flow}

\begin{tabular}{|c|c|}
\hline Were Xpert sample and culture obtained from same specimen? & $\begin{array}{l}1-\text { Yes } \\
2-\text { No } \\
9-\text { Unknown/NR }\end{array}$ \\
\hline $\begin{array}{l}\text { What specimen processing procedure was used before testing } \\
\text { with Xpert? }\end{array}$ & $\begin{array}{l}1 \text { - None } \\
2 \text { - NALC-NaOH } \\
3 \text { - NaOH (Petroff) } \\
4 \text { - Other } \\
9 \text { - Unknown/NR }\end{array}$ \\
\hline Was microscopy used? & $\begin{array}{l}1-\text { Yes } \\
2-\text { No } \\
9-\text { Unknown/NR }\end{array}$ \\
\hline Type of microscopy used & $\begin{array}{l}1 \text { - Ziehl-Neelsen } \\
2 \text { - Fluorescence microscopy } \\
3 \text { - Both Ziehl-Neelsen and fluorescence microscopy } \\
9 \text { - Unknown/NR }\end{array}$ \\
\hline $\begin{array}{l}\text { Smear type (if study used both direct and concentrated, } \\
\text { select concentrated) }\end{array}$ & $\begin{array}{l}1 \text { - Direct } \\
2 \text { - Concentrated (processed) } \\
9-\text { Unknown/NR }\end{array}$ \\
\hline $\begin{array}{l}\text { For Xpert specimen, what was the condition of the } \\
\text { specimen when tested? }\end{array}$ & $\begin{array}{l}1-\text { Fresh } \\
2 \text { - Frozen } \\
3 \text { - Both fresh and frozen } \\
9-\text { Unknown/NR }\end{array}$ \\
\hline
\end{tabular}

\section{Results}

Did the study report \% contaminated cultures?

(Enter percentage contaminated cultures, if

provided):
1 - Yes -> \% contaminated cultures:

$2-$ No

\section{\# of contaminated cultures/Total \# cultures performed = $\%$}


(Continued)

Did the study report the number of uninterpretable

results for Xpert for tuberculosis detection? (invalid, error, no result)

The uninterpretable rate for detection of PTB is the

number of tests classified as "invalid," "error," or "no result"

divided by the total number of Xpert tests performed.

Did the study report the number of indeterminate results for

Xpert for rifampicin resistance detection?

The indeterminate rate for detection of rifampicin resistance

was the number of tests classified as "MTB detected; Rif

resistance INDETERMINATE" divided by the total number

of Xpert-MTB positive results

Did the study report any Xpert rifampicin resistant positive

results in culture negative specimens?

Did the study report nontuberculous mycobacteria (NTM)?

Record number NTM over the number of cultures performed

If NTMs were identified, record number of Xpert positive

results among NTMs
1 - Yes -> \# Uninterpretable results:

Denominator is total number of Xpert tests performed

(Add total from Table 1 plus \# of uninterpretable results):

$2-$ No
1 - Yes ->\# Indeterminate results:

(Enter 0 indeterminate results if the total number in Table $6=$ the number of TPs in Table1)

Denominator is total number of Xpert tests performed

(Total Xpert positive results from Table 1 first row):

$2-$ No

1 - Yes -> Number reported:

$2-$ No

1 - Yes -> Number reported:

$2-$ No
\#Xpert positive tests among total number NTMs:

9 - Unknown/NR

Abbreviations: HIV: human immunodeficiency virus; LJ: Löwenstein-Jensen; MGIT: mycobacterial growth indicator tube; NR: Not reported; NTM: Nontuberculous mycobacteria; PTB: pulmonary tuberculosis.

\section{TABLES, examples}

Table 1.

(Continued)

\begin{tabular}{lll} 
(Continued) & & \multicolumn{2}{c}{ Confirmed tuberculosis } \\
\cline { 3 - 3 } Tuberculosisdetection, all participants & & Yes \\
\hline Xpert MTB/RIF result & & No \\
\cline { 2 - 3 } & Positive & \\
\cline { 2 - 3 } & Negative \\
\hline & Total
\end{tabular}

Table 2 
Table 4.

(Continued)

\section{Rifampicin resistance detection}

\section{Appendix 4. Rules for QUADAS-2}

In QUADAS-2, we assessed methodological quality separately for each of the objectives, Xpert for pulmonary tuberculosis (PTB) detection and Xpert for rifampicin resistance detection.

\section{Domain 1: Patient selection}

\section{Xpert MTB/RIF or Xpert Ultra for PTB detection}

Risk of bias: Could the selection of patients have introduced bias?

Signalling question 1: Was a consecutive or random sample of patients enrolled? We answered 'yes' if the study enrolled a consecutive or random sample of eligible patients; 'no' if the study selected patients by convenience; and 'unclear' if the study did not report the manner of patient selection or we could not tell. 
Signalling question 2: Was a case-control design avoided? Studies using a case-control design were not included in the review because this study design, especially when used to compare results in severely ill patients with those in relatively healthy individuals, may lead to overestimation of accuracy in diagnostic studies. We answered 'yes' for all studies.

Signalling question 3: Did the study avoid inappropriate exclusions? We answered 'yes' if the study included both smear-positive and smearnegative individuals; 'no' if the study included primarily or exclusively smear-positive or smear-negative patients; and 'unclear' if we could not tell. We also answered 'no' if the study included primarily or exclusively patients who had undergone previous treatment (retreatment patients).

Applicability: Are there concerns that the included patients and setting do not match the review question?

We were interested in how Xpert MTB/RIF or Xpert Ultra performed in patients who were evaluated as they would be in routine practice. We answered 'low concern' if patients were evaluated in local hospitals or primary care centres. We answered 'high concern' if patients were evaluated exclusively as inpatients in tertiary care centres. We answered 'unclear concern' if the clinical setting was not reported or there was insufficient information to make a decision. We also answered 'unclear concern' if Xpert MTB/RIF or Xpert Ultra testing was done at a central-level laboratory and the clinical setting was not reported for the following reason. It was difficult to tell if a given reference laboratory provided services mainly to very sick patients.

\section{Xpert MTB/RIF or Xpert ultra for rifampicin resistance detection}

Domain 1: Patient selection is the same as for Xpert for PTB detection except for

Signalling question 3: Did the study avoid inappropriate exclusions? We answered 'yes' if the study included both smear-positive and smearnegative individuals; 'no' if the study included primarily or exclusively smear-positive or smear-negative patients; and 'unclear' if we could not tell. We answered 'yes' if the study included primarily or exclusively retreatment patients because the group at risk for rifampicin resistance includes patients who had undergone previous treatment.

\section{Domain 2: Index test}

\section{Xpert for PTB detection}

Risk of bias: Could the conduct or interpretation of the index test have introduced bias?

Signallingquestion 1: Were the index test results interpreted without knowledge of the results of the reference standard? We answered this question 'yes' for all studies because Xpert test results were automatically generated and the user was provided with printable test results. Thus, there is no room for subjective interpretation of test results.

Signallingquestion 2: If a threshold was used, was it prespecified? The threshold was prespecified in all versions of Xpert. We answered this question 'yes' for all studies.

For risk of bias, we judged 'low concern' for all studies.

Applicability: Are there concerns that the index test, its conduct, or its interpretation differ from the review question? Variations in test technology, execution, or interpretation may affect estimates of the diagnostic accuracy of a test. All steps in the Xpert MTB/RIF and Xpert Ultra assays are completely automated and self-contained following sample loading. We answered 'low concern' if the index test was performed as recommended by the manufacturer, which was true for most studies. We answered 'unclear concern' if the ratio of the Xpert MTB/RIF or Xpert Ultra sample reagent: specimen volume was not 2:1 for a raw specimen or 3:1 for a sediment, as recommended by the manufacturer. Central-level laboratories use more highly trained staff than peripheral and intermediate-level laboratories. However, we did not consider this to be a concern about applicability because, in some studies, the reason Xpert MTB/RIF or Xpert Ultra was performed in a central-level laboratory was the requirement for a sophisticated laboratory infrastructure to perform culture (reference standard) not to perform Xpert.

\section{Xpert for rifampicin resistance detection}

Domain 2: Index test is the same as for Xpert for PTB detection.

\section{Domain 3: Reference standard}

\section{Xpert for PTB detection}

Risk of bias: Could the reference standard, its conduct, or its interpretation have introduced bias?

Signallingquestion 1: Is the reference standard likely to correctly classify the target condition?

We answered 'yes' for all studies, since culture as a reference standard was a criterion for inclusion in the review.

Signallingquestion 2: Were the reference standard results interpreted without knowledge of the results of the index test? 
We answered 'yes' if the reference test provided an automated result (for example, MGIT 960), blinding was explicitly stated, or it was clear that the reference standard was performed at a separate laboratory and/or performed by different people. We answered 'no' if the study stated that the reference standard result was interpreted with knowledge of the Xpert MTB/RIF or Xpert Ultra test result. We answered 'unclear' if we could not tell.

Applicability: Are there concerns that the target condition as defined by the reference standard does not match the question? We answered 'high concern' if included studies did not speciate mycobacteria isolated in culture; 'low concern' if speciation was performed; and 'unclear concern' if we could not tell.

\section{Xpert for rifampicin resistance detection}

Risk of bias: Could the selection of patients have introduced bias?

Signallingquestion 1: Is the reference standard likely to correctly classify the target condition?

We answered 'yes' if either culture-based drug susceptibility testing (DST) or MTBDRplus was used. These were criteria for inclusion for this objective of the review.

\section{Signallingquestion 2: Were the reference standard results interpreted without knowledge of the results of the index test?}

We answered 'yes' if the reference test provided an automated result (for example, MGIT 960), blinding was explicitly stated, or it was clear that the reference standard was performed at a separate laboratory and/or performed by different people. We answered 'no' if the study stated that the reference standard result was interpreted with knowledge of the Xpert MTB/RIF or Xpert Ultra test result. We answered 'unclear' if we could not tell.

Applicability: Are there concerns that the target condition as defined by the reference standard does not match the question? We judged applicability to be of 'low concern' for those studies evaluating Xpert for rifampicin resistance because these specimens had already been identified as Mycobacterium tuberculosis positive.

\section{Domain 4: Flow and timing}

\section{Xpert for PTB detection}

Risk of bias: Could the patient flow have introduced bias?

Signallingquestion 1: Was there an appropriate interval between the index test and reference standard? In most included studies, we expected that specimens for Xpert MTB/RIF or Xpert Ultra and culture would be obtained at the same time, when patients were evaluated for presumptive PTB. However, even if there were a delay of several days between index test and reference standard, tuberculosis is a chronic disease and we considered misclassification of disease status to be unlikely, as long as treatment was not initiated in the interim. We answered 'yes' if the index test and reference standard were performed at the same time or if the time interval was less than or equal to seven days, 'no' if the time interval is greater than seven days, and 'unclear' if we could not tell.

Signallingquestion 2: Did all patients receive the same reference standard? We answered this question 'yes' for all studies as an acceptable reference standard (either solid or liquid culture) was specified as a criterion for inclusion in the review. However, we acknowledge that it is possible that some specimens could undergo solid culture and others liquid culture. This could potentially result in variations in accuracy, but we thought the variation would be minimal.

Signallingquestion 3: Were all patients included in the analysis? We determined the answer to this question by comparing the number of patients enrolled with the number of patients included in the $2 \times 2$ tables. We answered ' $y e s$ ' if the numbers matched and 'no' if there were patients enrolled in the study that were not included in the analysis. We answered 'unclear' if we could not tell.

\section{Xpert for rifampicin resistance detection}

Domain 4: Flow and timing is the same as for Xpert MTB/RIF or Xpert Ultra for PTB detection.

Judgements for 'risk of bias' assessments for a given domain

- If we answered all signalling questions for a domain 'yes', then we judged risk of bias as 'low'.

- If we answered all or most signalling questions for a domain 'no', then we judged risk of bias as 'high'.

- If we answered only one signalling question for a domain 'no', we discussed further the 'Risk of bias' judgement.

- If we answered all or most signalling questions for a domain 'unclear', then we judged risk of bias as 'unclear'.

- If we answered only one signalling question for a domain 'unclear', we discussed further the 'Risk of bias' judgement for the domain. 


\section{Appendix 5. Statistical appendix}

\section{Bayesian bivariate hierarchical model}

The Bayesian bivariate hierarchical model used for the meta-analyses is summarized below. The hierarchical framework took into account heterogeneity between studies and also between centres within two of the largest studies. The model was derived as an extension of previously described models (Chu 2009; Reitsma 2005). An OpenBUGS program to fit this model is provided below. Three independent, dispersed sets of starting values were used to run separate chains. The Gelman-Rubin statistic within the OpenBUGS program was used to assess convergence. No convergence problems were observed. The first 10,000 iterations were treated as burn-in iterations and dropped. Summary statistics were obtained based on a total of 150,000 iterations resulting from the three separate chains.

Notation: From the jth centre in the ith study we extracted the cross-tabulation between the index and reference tests TPij, FPij, TNij, FNij. The sensitivity in ijth study is denoted by Sij and the specificity by SPij. We denote the Binomial probability distribution with sample size $\mathrm{N}$ and probability $\mathrm{p}$ as Binomial $(\mathrm{p}, \mathrm{N})$, the Bivariate Normal probability distribution with mean vector $\mu$ and variance-covariance matrix $\Sigma$ as $\operatorname{BVN}(\mu, \Sigma)$, the univariate Normal distribution with mean $\mathrm{m}$ and variance $\mathrm{s}$ by $\mathrm{N}(\mathrm{m}, \mathrm{s})$ and the Uniform probability distribution between $a$ and $b$ by Uniform $(a, b)$.

Likelihood Figure 22

Figure 22. Bayesian bivariate hierarchical model, likelihood.

\section{Centre-level:}

For studies with only 1 centre:

\section{$\mathrm{TP}_{\mathrm{i} 1} \sim \operatorname{Binomial}\left(\mathrm{S}_{\mathrm{i}}, \mathrm{TP}_{\mathrm{i} 1}+\mathrm{FN}_{\mathrm{i} 1}\right), \mathrm{TN}_{\mathrm{i} 1} \sim \operatorname{Binomial}\left(\mathrm{SP}_{\mathrm{i}}, \mathrm{TN}_{\mathrm{i} 1}+\mathrm{FP}_{\mathrm{i} 1}\right)$}

Formulticentre studies:

$\mathrm{TP}_{\mathrm{ij}} \sim \operatorname{Binomial}\left(\mathrm{S}_{\mathrm{ij}}, \mathrm{TP}_{\mathrm{ij}}+\mathrm{FN}_{\mathrm{ij}}\right), \mathrm{TN}_{\mathrm{ij}} \sim \operatorname{Binomial}\left(\mathrm{SP}_{\mathrm{ij}}, \mathrm{TN}_{\mathrm{ij}}+\mathrm{FP}_{\mathrm{ij}}\right)$

$\left(\begin{array}{c}\operatorname{logit}\left(S_{i j}\right) \\ \operatorname{logit}\left(S P_{i j}\right)\end{array}\right) \sim B V N\left(l_{i}, \Sigma_{\mathrm{i}}\right)$,

where $l_{i}=\left(\begin{array}{c}\operatorname{logit}\left(S_{i}\right) \\ \operatorname{logit}\left(S P_{i}\right)\end{array}\right)$ and $\Sigma_{i}=\left(\begin{array}{cc}\sigma_{i 1}^{2} & k_{i} \sigma_{i 1} \sigma_{i 2} \\ k_{i} \sigma_{i 1} \sigma_{i 2} & \sigma_{i 2}^{2}\end{array}\right)$

Study-level:

$\left(\begin{array}{c}\operatorname{logit}\left(S_{i}\right) \\ \operatorname{logit}\left(S P_{i}\right)\end{array}\right) \sim B V N\left(\mu=\left(\begin{array}{l}\mu_{1} \\ \mu_{2}\end{array}\right), T=\left(\begin{array}{cc}\tau_{1}^{2} & \rho \tau_{1} \tau_{2} \\ \rho \tau_{1} \tau_{2} & \tau_{2}^{2}\end{array}\right)\right)$ 
The pooled sensitivity is given by $1 / 1+\exp \left(-\mu_{1}\right)$ and pooled specificity as $1 / 1+\exp \left(\mu_{2}\right)$.

Prior distributions Figure 23.

Figure 23. Bayesian bivariate hierarchical model, prior distributions.

\section{$\mu_{1}$ and $\mu_{2} \sim N(0,100)$}

\section{$k_{i}$ and $\rho \sim U(-1,1)$}

\section{$\frac{1}{\sigma_{1}^{2}}, \frac{1}{\sigma_{2}^{2}}, \frac{1}{\tau_{1}^{2}}$ and $\frac{1}{\tau_{2}^{2}} \sim \operatorname{Gamma}($ shape $=2$, rate $=0.5)$}

Prior distributions were placed over the coefficients in the linear function: $a 1$ and $a 2^{\sim} N(0,4)$ and b1 and b2 $\sim N(0,1.39)(B u z o i a n u ~ 2008)$.

\# BIVARIATE MODEL ASSUMING PERFECT CULTURE REFERENCE TEST

\# ALLOWING FOR HETEROGENEITY BETWEEN CENTRES WITHIN TWO OF

\# THE STUDIES (BOEHME 2010 and BOEHME 2011)

model \{

\#\#\#\#\#\#\#\#\#\#\#\#\#\#\#\#\#\#\#\#\#\#\#\#\#\# BOEHME 2010

for(j in 1:5) \{

$\operatorname{logit}(T P R . q[j])<-q 1[j, 1]$

$\operatorname{logit}(F P R . q[j])<--q 1[j, 2]$

$\operatorname{pos} 1[j]<-T P 1[j]+F N 1[j]$

neg1[j]<-TN1[j]+FP1[j]

TP1[j] dbin(TPR.q[j],pos1[j])

FP1[j] dbin(FPR.q[j],neg1[j])

se.q[j] <- TPR.q[j]

sp.q[j] <- 1-FPR.q[j]

q1[j,1:2] dmnorm(l[1,1:2], T1[1:2,1:2])

\}

T1[1:2,1:2]<-inverse(SIGMA1[1:2,1:2])

SIGMA1 $[1,1]<-\operatorname{sigma} 1[1]^{\star} \operatorname{sigma} 1[1]$ 
SIGMA1[2,2] <- sigma1[2]*sigma1[2]

SIGMA1[1,2] <- k1*sigma1[1] ${ }^{\star} \operatorname{sigma1[2]~}$

$\operatorname{SIGMA} 1[2,1]<-\mathrm{k}^{\star}$ sigma1[1] ${ }^{\star} \operatorname{sigma} 1[2]$

sigma1[1] <- pow(prec1[1],-0.5) \# replaced by sigma1[1] dunif(0,3) in sensitivity analysis to check impact of less informative prior sigma1[2] <- pow(prec1[2],-0.5) \# replaced by sigma1[2] dunif(0,3) in sensitivity analysis to check impact of less informative prior $\operatorname{prec1}[1]$ dgamma(2,0.5) \# replaced by prec1[1] <- pow(sigma1[1],-2) in sensitivity analysis to check impact of less informative prior $\operatorname{prec1[2]~~dgamma(2,0.5)~\# ~replaced~by~prec1[2]~<-~pow(sigma1[2],-2)~in~sensitivity~analysis~to~check~impact~of~less~informative~prior~}$ $\mathrm{k} 1$ dunif(-1,1)

se[1]<-1/(1+exp $(-1[1,1]))$

$\operatorname{sp}[1]<-1 /(1+\exp (l[1,2]))$

$\mathbb{I}[1,1: 2] \sim \operatorname{dmnorm}(\mathrm{mu}[1: 2], \mathrm{T}[1: 2,1: 2])$

\#\#\#\#\#\#\#\#\#\#\#\#\#\#\#\#\#\#\#\#\#\#\#\#\#\#\#\# BOEHME 2011

for(j in 1:6) \{

logit(TPR.r[j])<- r1[j,1]

$\operatorname{logit}(F P R . r[j])<--r 1[j, 2]$

$\operatorname{pos} 2[\mathrm{j}]<-\mathrm{TP} 2[\mathrm{j}]+\mathrm{FN} 2[\mathrm{j}]$

neg2 $[\mathrm{j}]<-$ TN2[j]+FP2[j]

TP2[j] dbin(TPR.r[j],pos2[j])

FP2[j] dbin(FPR.r[j],neg2[j])

se.r[j] <- TPR.r[j]

sp.r[j] <- 1-FPR.r[j]

$\mathrm{r} 1[\mathrm{j}, 1: 2]^{\sim}$ dmnorm(l[2,1:2], T2[1:2,1:2])

\}

T2[1:2,1:2]<-inverse(SIGMA2[1:2,1:2])

SIGMA2 $[1,1]<-\operatorname{sigma2}[1]^{\star} \operatorname{sigma2}[1]$

SIGMA2[2,2] <- sigma2[2] ${ }^{\star} \operatorname{sigma2[2]~}$

SIGMA2[1,2] <- k2* sigma2[1] ${ }^{*} \operatorname{sigma2[2]~}$

SIGMA2[2,1] <- k2*sigma2[1]*sigma2[2]

sigma2[1] <- pow(prec2[1],-0.5) \# replaced by sigma2[1] dunif(0,3) in sensitivity analysis to check impact of less informative prior sigma2[2] <- pow(prec2[2],-0.5) \# replaced by sigma2[2] dunif(0,3) in sensitivity analysis to check impact of less informative prior $\operatorname{prec2}[1] \sim$ dgamma(2,0.5) \# replaced by prec2[1] <- pow(sigma2[1],-2) in sensitivity analysis to check impact of less informative prior prec2[2] dgamma(2,0.5) \# replaced by prec2[2] <- pow(sigma2[2],-2) in sensitivity analysis to check impact of less informative prior 
$\mathrm{k} 2$ dunif(-1,1)

se[2]<-1/(1+exp $(-\mid[2,1]))$

$\operatorname{sp}[2]<-1 /(1+\exp (l[2,2]))$

$\mathbb{I}[2,1: 2] \sim \operatorname{dmnorm}(\mathrm{mu}[1: 2], \mathrm{T}[1: 2,1: 2])$

\#\#\#\#\#\#\#\#\#\#\#\#\#\#\#\#\#\#\#\#\#\#\#\#\#\#\# SINGLE CENTRE STUDIES

for(i in 3:70) \{

\#\#\#\#\#\#\#\#\#\#\#\#\#\#\#\#\#\#\#\#\#\#\#\#\#\# LIKELIHOOD

$\operatorname{logit}(\operatorname{TPR}[\mathrm{i}])<-l[\mathrm{i}, 1]$

$\operatorname{logit}(F P R[i])<--\mid[i, 2]$

$\operatorname{pos}[i]<-T P[i]+F N[i]$

neg $[\mathrm{i}]<-\mathrm{TN}[\mathrm{i}]+\mathrm{FP}[\mathrm{i}]$

$\operatorname{TP}[\mathrm{i}]$ $d b i n(T P R[i], \operatorname{pos}[i])$

$\mathrm{FP}[\mathrm{i}]$ dbin(FPR[i],neg[i])

se $[i]<-$ TPR $[i]$

$\mathrm{sp}[\mathrm{i}]<-1-\mathrm{FPR}[\mathrm{i}]$

l[i,1:2] dmnorm(mu[1:2], T[1:2,1:2])

\}

\#\#\#\#\#\#\#\#\#\#\#\#\#\#\#\#\#\#\#\#\#\#\#\#\#\#\#\# HYPER PRIOR DISTRIBUTIONS

$\mathrm{mu}[1]$ dnomr $(0.0 .25)$ \# replaced by $m u[1]$ dnorm $(0,0.01)$ in sensitivity analysis to check impact of less informative prior $\mathrm{mu}[2]$ dnomr $(0.0 .25)$ \# replaced by $m u[2] ~ \sim ~ d n o r m(0,0.01)$ in sensitivity analysis to check impact of less informative prior $\mathrm{T}[1: 2,1: 2]<-$ inverse $(\mathrm{TAU}[1: 2,1: 2])$

\#\#\#\# BETWEEN-STUDY VARIANCE-COVARIANCE MATRIX

$\operatorname{TAU}[1,1]<-\operatorname{tau}[1]^{\star} \operatorname{tau}[1]$

$\operatorname{TAU}[2,2]<-\operatorname{tau}[2]^{\star} \operatorname{tau}[2]$

$\operatorname{TAU}[1,2]<-\operatorname{rho}{ }^{\star} \operatorname{tau}[1]^{\star} \operatorname{tau}[2]$

$\operatorname{TAU}[2,1]<-\operatorname{rho}{ }^{*} \operatorname{tau}[1]^{*} \operatorname{tau}[2]$

$\operatorname{tau}[1]<-\operatorname{pow}(\operatorname{prec}[1],-0.5)$ \# replaced by tau[1] dunif(0,3) in sensitivity analysis to check impact of less informative prior $\operatorname{tau}[2]<-\operatorname{pow}(\operatorname{prec}[2],-0.5)$ \# replaced by tau[2] dunif(0,3) in sensitivity analysis to check impact of less informative prior \#\#\# prec = between-study precision in the logit(sensitivity)and logit(specificity)

prec[1] dgamma $(2,0.5)$ \# replaced by prec[1] <- powtau[1],-2) in sensitivity analysis to check impact of less informative prior prec[2] dgamma(2,0.5) \# replaced by prec[2] <- powtau[2],-2) in sensitivity analysis to check impact of less informative prior

Xpert MTB/RIF and Xpert MTB/RIF Ultra for pulmonary tuberculosis and rifampicin resistance in adults (Review) 
rho dunif(-1,1)

\#\#\#\#\#\#\#\#\#\#\#\#\#\#\#\#\#\#\#\#\#\#\#\#\#\#\# OTHER PARAMETERS OF INTEREST

\#\#\#\# POOLED SENSITIVITY AND SPECIFICITY

Pooled_S<-1/(1+exp(-mu[1]))

Pooled_C $<-1 /(1+\exp (-\mathrm{mu}[2]))$

\#\#\#\# PREDICTED SENSITIVITY AND SPECIFICITY IN A FUTURE STUDY

I.new[1:2] dmnorm(mu[],T[,])

sens.new <- $1 /(1+\exp (-\operatorname{lnew}[1]))$

spec.new <- 1/(1+exp(-l.new[2]))

\}\#\#\#\# END OF PROGRAM

\#\#\#\#\#\#\#\#\#\#\#\#\#\#\#\#\#\#\#\#\#\#\#\#\#\#\#\#\#\#\#\#\#\#\#\#\#\#\#\#\#\#\#\#\#\#\#\#\#\#\#\#\#\#\#\#\#\#\#\#\#\#\#\#\#

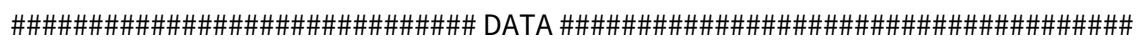

\# DATA WAS READ FROM THREE SEPARATE FILES

\# DATA 1 - BOEHME 2010

TP1[] FP1[] FN1[] TN1[]

12382491

20118105

136910188

3677257

1791840

END

\#row 1 : Azerbaijan

\#row 2 : Peru

\#row 3 : South Africa, Cape Town

\#row 4 : South Africa, Durban

\#row 5 : India

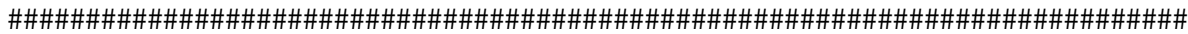

\# DATA 2 - FROM BOEHME 2011

TP2[] FP2[] FN2[] TN2[]

203426303

17136825

201232669

121024144

101160671

136512234

END

\#row 1 : Azerbaijan

\#row 2 : Peru

\#row 3 : South Africa

\#row 4 : Uganda

\#row 5 : India

\#row 6 : The Philippines 
TP[] FP[] FN[] TN[]

NA NA NA NA

NA NA NA NA

323204

807110

1611770

1111147

811341677

19114455

212566

2758155

\#...

\# DATA HAVE BEEN TRUNCATED FOR EASE OF PRESENTATION IN THIS APPENDIX

\# THE COMPLETE DATA CAN BE FOUND IN Figure 10

$\#$...

8958234

11114475

941029291

681513129

1111930320

1542731517

197630180

848791006

310468

601110

END

\# row 1 Boheme 2010

\# row 2 Boheme 2011

\# row 3 Adelman 2015

\# row 4 Al-darraji 2013

\# row 5 Atwebembeire 2016

\# row 6 Balcells 2012

\# row 7 Balcha 2014

\# row 8 Barmankulova 2015

\# row 9 Bates 2013

\# row 10 Bjerrum 2016

\#...

\# DATA HAVE BEEN TRUNCATED FOR EASE OF PRESENTATION IN THIS APPENDIX

\# THE COMPLETE DATA CAN BE FOUND IN Figure 10

$\#$...

\# row 60 Sharma 2015

\# row 61 Shenai 2016

\# row 62 Sohn 2014

\# row 63 Ssengooba 2014

\# row 64 Tang 2017

\# row 65 Theron 2011

\# row 66 Theron 2014

\# row 67 Tsuyuguchi 2017

\# row 68 Yoon 2017

\# row 69 Zeka 2011

\# row 70 Zmak 2013

\section{Appendix 6. Bayesian bivariate hierarchical model}

Figure 22 Bayesian bivariate hierarchical model, likelihood

Figure 23 Bayesian bivariate hierarchical model, prior distributions 


\section{FEEDBACK}

\section{Boyles, 7 October 2014}

\section{Summary}

Name: Tom Boyles

Affiliation: University of Cape Town

I certify that I have no affiliations with or involvement in any organization or entity with a financial interest in the subject matter of my feedback.

In the initial version of Steingart et al's systematic review of the Xpert ${ }^{\circledR}$ MTB/RIF assay for pulmonary tuberculosis and rifampicin resistance in adults (Steingart 2013) includes 15 studies where Xpert MTB/RIF was used as an initial test replacing smear microscopy, with the majority of patients being drawn from two major studies (Boehme 2010, Boehme 2011). My comment relates to the appropriate reference standard for tuberculosis is these studies. The systematic review appraised the quality of included studies with the Quality Assessment of Diagnostic Accuracy Studies (QUADAS-2) (Whiting 2011) tool which states that estimates of test accuracy are based on the assumption that the reference standard is $100 \%$ sensitive and that specific disagreements between the reference standard and index test result from incorrect classification by the index test.

For each of the studies in question the reference standard for tuberculosis is listed as "Löwenstein-Jensen culture and MGIT 960" and the review considers that the reference standard is likely to correctly classify the target condition. There is considered to be low risk of bias or applicability concerns relating to the reference test.

However, in Boehme et al 2010 there were 105 patients with 'clinical tuberculosis' who were excluded from the analysis. These patients were negative by the reference standard of Löwenstein-Jensen culture and MGIT 960 and should have been included in the 'no tuberculosis' group. In Boehme et al 2011 there were 153 similar patients who were excluded from the analysis.

Neither paper gives justification for the exclusion of these patients who according to QUADAS-2 were negative by the reference standard and should be included in the 'no tuberculosis' group. Ideally the systematic review should be amended to include these patients but if the data is unavailable the risk of bias should be acknowledged.

Note from the Editors: In addition to the above feedback, Boyles et al. published a case study in The International Journal of Tuberculosis and Lung Disease which outlined the above arguments, and illustrates this with a case study (Boyles 2014); which the Cochrane authors respond to, in the same journal (see below).

\section{Reply}

The review authors thank Boyles et al. for this comment. They raise important points about the selective exclusion of culture negative clinical tuberculosis cases in the Boehme studies.

We considered the published case study (Boyles 2014) in detail, and in response we carried out additional analyses to determine whether the Boehme studies unduly influenced the overall findings of this Cochrane review. One way we did this was by repeating the meta-analysis with studies for which we could extract data for all enrolled participants, including patients classified as 'clinical tuberculosis' with negative sputum culture. We considered these participants as not having tuberculosis. In the new analysis, we found pooled sensitivity and specificity estimates to be similar to those we previously reported.

We published our findings as a response to Boyles et al. in The International Journal of Tuberculosis and Lung Disease (Steingart 2015).

In the updated Cochrane Review, for Boehme 2010, we included culture negative results (clinical tuberculosis cases) in determinations of Xpert MTB/RIF specificity. For Boehme 2011, we did not have data for clinical tuberculosis, and therefore, in the Flow and Timing domain, we changed our judgement for risk of bias to 'high'.

\section{WHAT'S NEW}

\begin{tabular}{lll}
\hline Date & Event & Description \\
\hline 5 June 2019 & $\begin{array}{l}\text { New citation required but conclusions } \\
\text { have not changed }\end{array}$ & $\begin{array}{l}\text { The findings in this update are consistent with those reported } \\
\text { previously (Steingart 2014). }\end{array}$ \\
\hline 5 June 2019 & New search has been performed & $\begin{array}{l}\text { The review authors identified 95 unique studies, integrating } 77 \\
\text { new studies since publication of the Cochrane Review (Steingart } \\
\text { 2014). }\end{array}$
\end{tabular}




\section{HISTORY}

Protocol first published: Issue 1, 2012

Review first published: Issue 1, 2013

\begin{tabular}{lll}
\hline Date & Event & Description \\
\hline 30 June 2015 & Amended & $\begin{array}{l}\text { Added revised data including (smear positive culture negatives) } \\
\text { for Boehme 2010 and Rachow. Added corrected data for Hanra- } \\
\text { han. Added test and analysis for Hx of TB. Amended patient se- } \\
\text { lection for Boehme 2011 to high risk of bias. }\end{array}$ \\
\hline
\end{tabular}

\begin{tabular}{lll}
\hline 16 March 2015 & Feedback has been incorporated & $\begin{array}{l}\text { Feedback from Dr Tom Boyles at University of Cape Town has } \\
\text { been incorporated and responded to. }\end{array}$
\end{tabular}

\begin{tabular}{ll}
\hline Amended 2014 & $\begin{array}{l}\text { Following information from one of the trial authors, details of } \\
\text { the version of Xpert MTB/RIF used in Balcells } 2012 \text { have been cor- } \\
\text { rected. }\end{array}$
\end{tabular}

13 February $2014 \quad$ Amended

Sentence moved in abstract; corrected 'pooled median sensitivity' to 'median pooled sensitivity' throughout.

30 November $2013 \quad$ New search has been performed

\section{We performed an updated literature search on 7 February 2013.}

2. For smear microscopy as a comparator test, we added a descriptive plot showing the estimates of sensitivity and specificity of Xpert compared with those of smear microscopy in studies that reported on both tests.

3. We included studies using Xpert version G4 (two studies) and studies evaluating Xpert in primary care clinics (two studies). These studies did not change the overall findings.

4. We improved the QUADAS-2 assessment concerning applicability.

5. For TB detection, we repeated our earlier meta-regression analyses within subgroups defined by smear status.

6 . For rifampicin resistance detection, we performed univariate meta-analyses for sensitivity and specificity separately in order to include studies in which no rifampicin resistance was detected. We also performed a sensitivity analysis using the bivariate random-effects model for the subset of studies that provided data for both sensitivity and specificity.

7. We revised the summary of findings table to include clinical scenarios with prevalence levels recommended by the World Health Organization.

8. In the Background, we shortened the section on alternative tests to include only those tests most relevant to the review.

9. We added health economic considerations to the Discussion.

10. We added updated TB surveillance information.

\begin{tabular}{lll}
\hline 30 November 2013 & $\begin{array}{l}\text { New citation required but conclusions } \\
\text { have not changed }\end{array}$ & We conducted a new search and revised the review as described. \\
\hline 17 January 2013 & Amended & $\begin{array}{l}\text { We made some minor edits to the text to correct typographical } \\
\text { errors. In addition, we replaced Figures 6, 8, 11, and 13 with new } \\
\text { figures with minor modifications to the prediction regions. }\end{array}$
\end{tabular}




\section{CONTRIBUTIONS OF AUTHORS}

MP conceived the original idea for the review.

$\mathrm{KRS}, \mathrm{MP}$, and ND wrote the original protocol.

For this updated Cochrane Review, Vittoria Lutje designed the search strategy.

DJH, MK, JSZ, DT, and KRS assessed articles for inclusion and extracted data.

MK and JSZ managed REDCap.

DJH, MK, IS, ND, and KRS analysed the data and interpreted the analyses.

DJH, MK, IS, ND, and KRS drafted the manuscript. In particular, IS and ND drafted the statistical analysis section and the statistical appen-

dix. EAO drafted the section on patient health outcomes.

SGS and MP provided critical comments to the manuscript.

All authors read and approved the final manuscript draft.

\section{DECLARATIONSOF INTEREST}

DJH received financial support for the submitted work from McGill University.

MK has no known conflicts of interest.

JSZ has no known conflicts of interest.

IS has no known conflicts of interest.

ND has no known conflicts of interest.

DT has no known conflicts of interest.

SGS is employed by the Foundation for Innovative New Diagnostics (FIND). FIND has conducted studies and published on Xpert MTB/RIF as part of a collaborative project between FIND, a Swiss non-profit, Cepheid, a US company, and academic partners. The product developed through this partnership was developed under a contract that obligated FIND to pay for development costs and trial costs and Cepheid to make the test available at specified preferential pricing to the public sector in low- and middle-income countries. In addition, FIND conducted studies for the Xpert MTB/Rif Ultra assay, which have also been published.

EAC has no known conflicts of interest.

MP serves on the Scientific Advisory Committee of FIND, Geneva. FIND is a non-profit agency that works on global health diagnostics.

KRS received financial support for the submitted work from McGill University, and has received financial support for the preparation of systematic reviews and educational materials, consultancy fees from FIND (for the preparation of systematic reviews), honoraria, and travel support to attend WHO guideline meetings.

The authors have no financial involvement with any organization or entity with a financial interest in or financial conflict with the subject matter or materials discussed in the review apart from those disclosed.

\section{SOURCES OF SUPPORT}

\section{Internal sources}

- Liverpool School of Tropical Medicine, UK.

\section{External sources}

- United States Agency for International Development (USAID), USA.

Development and publication of this manuscript was in part made possible with financial support from the USAID

- Department for International Development (DFID), UK.

Project number 300342-104

\section{DIFFERENCES BETWEEN PROTOCOL AND REVIEW}

In the protocol we stated that we would extract data on industry sponsorship. However, we became aware that FIND had negotiated a special price for the assay for tuberculosis-endemic countries. As most of the included studies were located in tuberculosis-endemic countries, we assumed Xpert had been purchased at the negotiated price. We therefore did not consider the included studies to be sponsored by industry. 
We stated we would discuss the consequences when an uninterpretable test result was considered to be a (false) true negative result (may lead to missed or delayed diagnosis, with potential for increased morbidity, mortality, and tuberculosis transmission), or considered to be a (false) true positive result (may lead to unnecessary treatment with adverse events and increased anxiety). Since the rate of uninterpretable results was very low, we did not discuss these consequences.

\section{NDEX TERMS}

\section{Medical Subject Headings (MeSH)}

*Antibiotics, Antitubercular [pharmacology]; *Drug Resistance, Bacterial; *Mycobacterium tuberculosis [drug effects]; *Rifampin [pharmacology]; *Tuberculosis, Pulmonary [drug therapy]; Microbial Sensitivity Tests; Sensitivity and Specificity

\section{MeSH check words}

Humans 